UNITED STATES DEPARTMENT OF THE INTERIOR

GEOLOGICAL SURVEY

\title{
Analytical results and saple locality map of the nonmagnetic, heavy-mineral-concentrate samples collected from the eastern part of the Lime Hills quadrangle, Alaska
}

By

M.J. Malcolm, M.S. Allen*, and K.E. Slaughter*

Open-File Report 90-68

This report is preliminary and has not been reviewed for conformity with U.S. Geological Survey editorial standards and stratigraphic nomenclature. Any use of trade names is for descriptive purposes only and does not imply endorsement by the USGS.

*U.S. Geological Survey, DFC, Box 25046, MS 973, Denver, CO 80225 


\section{CONTENTS}

Page

Studies Related to AMRAP................................ 1

Introduction....................................... 1

General Geology...................................... 3

Methods of study.................................... 3

Sample Media........................................ 3

Sample Collection.................................. 4

Sample Preparation................................ 4

Sample Analysis................................... 4

Data Storage System................................... 5

Description of Data Table.............................. 5

Acknowledgments................................... 5

References Cited.................................... 6

\section{ILLUSTRATIONS}

Figure 1. Index map of the Lime Hills quadrangle, Alaska............ 2

Plate 1. Map showing location of heavy-mineral-concentrate samples,

Lime Hills quadrangle, Alaska.......................... in pocket

\section{TABLES}

Table 1. Limits of determination for spectrographic analysis of nonmagnet ic, heavy-mineral-concentrate samples............... 7

Table 2. Geochemical data for nonmagnetic, heavy-mineral-concentrate samples, Lime Hills quadrangle, Alaska. 


\section{STUDIES RELATED TO AMRAP}

The U.S. Geological Survey is required by the Alaska National Interests Lands Conservation Act (Publ ic Law 96-487, 1980) to survey certain Federal lands to determine the ir mineral potential. Results from the Alaska Mineral Resource Assessment Program (AMRAP) must be made available to the public and submitted to the President and Congress. This report is one of a series of publications that presents geochemical and mineralogical results collected from the mineral assessment study of the Lime Hills 1:250,000 scale quadrangle, Alaska. The geochemical data for nonmagnetic, heavy-mineralconcentrate samples from the eastern portion of the Lime Hills quadrangle are presented in this report.

\section{INTRODUCTION}

During the summers of 1987-88, a reconnaissance geochemical survey was conducted in the Lime Hills quadrangle, Alaska (fig. 1). The quadrangle is bounded by 1 atitude $61^{\circ} \mathrm{N}$. to $62^{\circ} \mathrm{N}$. and by longitude $153^{\circ} \mathrm{W}$. to $156^{\circ} \mathrm{W}$. The Lime Hills quadrangle comprises approximately $7,000 \mathrm{mi}^{2}\left(18,000 \mathrm{~km}^{2}\right)$, although the area covered by this repgrt represents only the eastern portion of the quadrangle (approx. 4,000 $\mathrm{mi}^{2} ; 10,000 \mathrm{~km}^{2}$ ) that is occupied by the Alaska Range and the Lyman Hills. Therefore, this report presents results of a geochemical survey that is still ongoing, with completion expected in 1991 . This interim report is deemed necessary due to the significance of the findings to be presented. In addition, the difference in geologic and physiographic character between the eastern portion of the quadrangle occupied by the Alaska Range and the western portion occupied by the lowlands of the Kuskokwim drainage basin warrants a separate interpretation of the geochemical data collected from each.

The portion of the quadrangle occupied by the Alaska Range is dominated by rugged, north-south trending ridges 4,000 to $7,000 \mathrm{ft}(1,200$ to $2,100 \mathrm{~m})$ in elevation which rise abruptly from the lower terrain to the west. These ridges connect extremely rugged snowcapped peaks more than $9,000 \mathrm{ft}(2,750 \mathrm{~m})$ in elevation, the highest being Mount Hesperus $(9,228 \mathrm{ft} ; 2813 \mathrm{~m})$. Broad glaciated valleys with floors generally less that $3,000 \mathrm{ft}(915 \mathrm{~m})$ in elevation lie between the ridges. On the western flank of the range rolling hills and glacial pediments dominate the terrain. Due to the mountainous topography, the Lyman Hills are also included in this report. Vegetation in the Alaska Range and on its flanks varies from barren mountain peaks to arctic tundra in glacial valleys, and northern latitude forest in lower valleys and lowlands west of the Alaska Range.

There is no road access to the Lime Hills quadrangle. The nearest reliable source of supplies is Anchorage, $130 \mathrm{mi}(200 \mathrm{~km})$ to the east. Only two sites of year-round habitation are located in the quadrangle, the Lime Village native settlement on the Stony River and the Sparrevohn U.S. Air Force Station in the southwest portion of the area. Improved airstrips capable of accommodating large freight-hauling aircraft (e.g. C-130) are present at each of these sites. Unimproved airstrips and lakes that can accommodate small aircraft occur scattered throughout the quadrangle, though sites for landing in the Alaska Range are few. Approximately 14 percent of the quadrangle lies within the Lake Clark National Park and Preserve and is included in this study. 


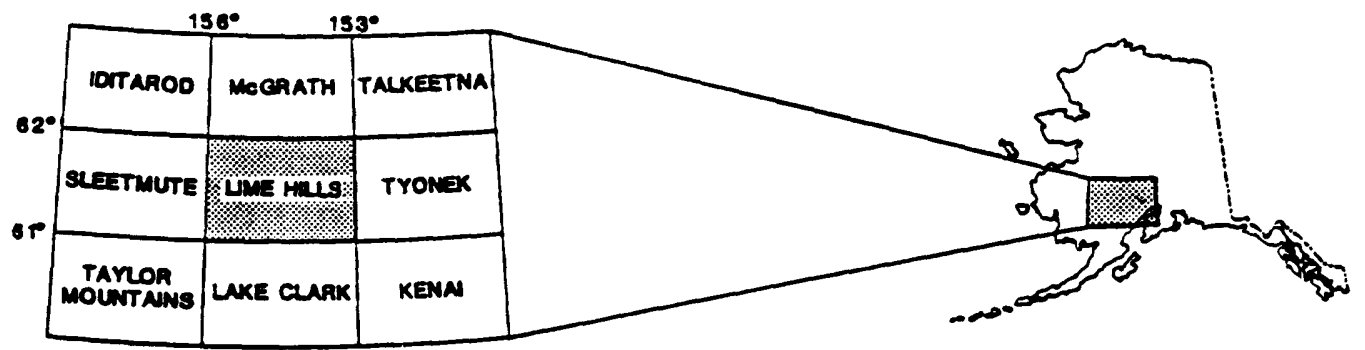

ALASKA

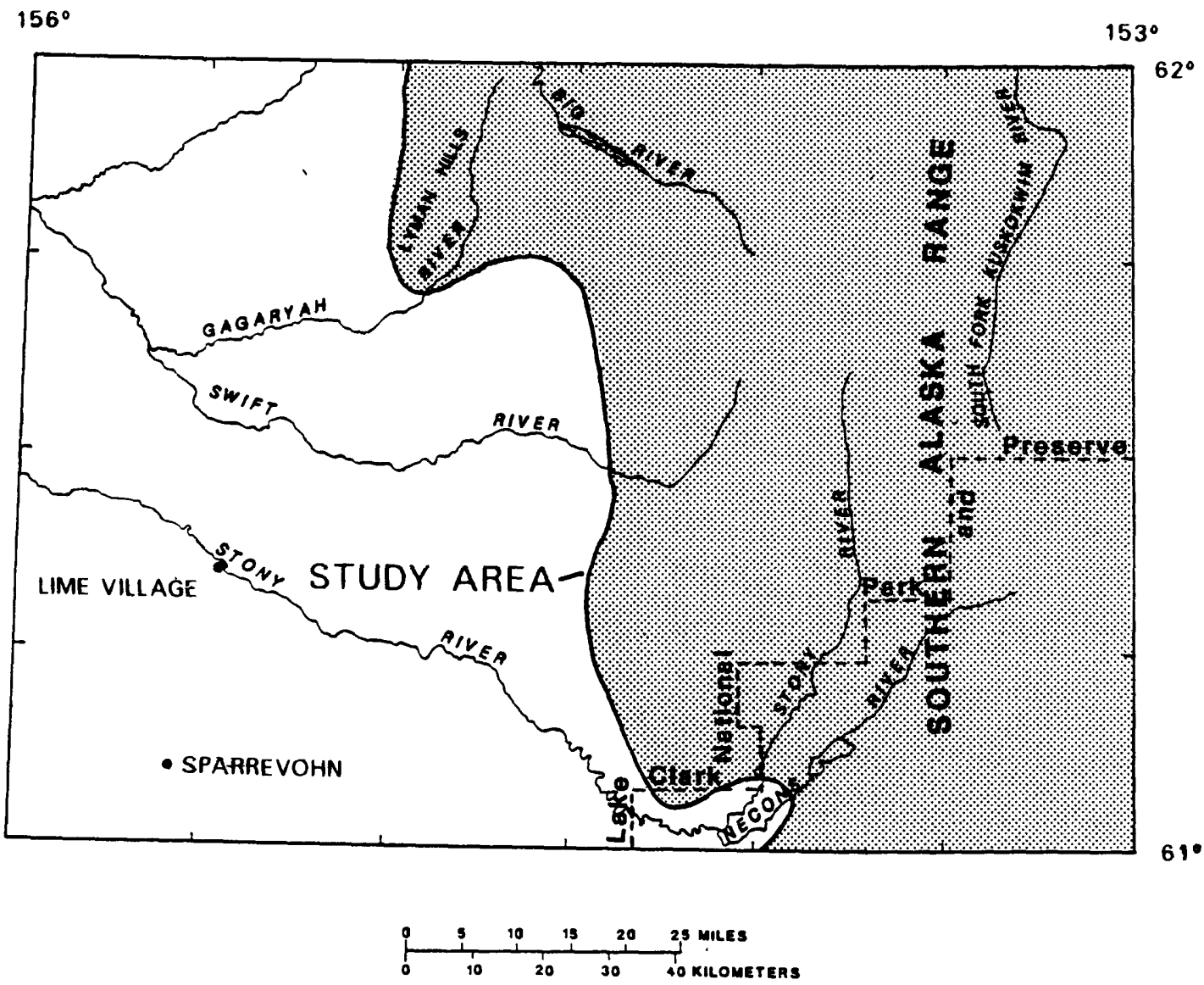

Figure 1. Index map of the Lime Hills quadrangle, Alaska 


\section{GENERAL GEOLOGY}

Several major geologic features have been identified in the Lime Hills quadrangle including parts of the Dillinger, Nixon Fork, and Kahiltna lithotectonic terranes, the Kuskokwim Group sedimentary rocks and the Alaska-Aluetian batholith.

The Nixon Fork terrane (Jones and others, 1984) is a shallow-water carbonate platform and clast ic sequence of Cambrian to Devonian age (Churkin, 1984; Blodgett and Clough, 1985). The Kuskokwim Group (Cady and others, 1955) is a sequence of deep to shallow marine, to non-marine clastic rocks of Early to Late Cretaceous age that unconformably overlie rocks of the Dillinger terrane. The rocks of the Nixon Fork terrane and Kuskokwim Group are present in the southwest portion of the quadrangle and are not included in the area covered by the present survey.

The Dillinger terrane, exposed in the northwest and north-central parts of the quadrangle consists of Cambrian through Devonian sedimentary rocks (Jones and others, 1984). The terrane is represented by a shallowing sequence of graptolitic shale, basinal carbonates, calcareous sandstones with minor chert and conglomerate, deposited in basinal, turbidite fan, and foreslope environments (Churkin, 1984; Bundtzen and others, 1987). The rocks of this terrane were isoclinally folded before the Jurassic period (Reed and Nelson, 1980).

The Kahiltna terrane (Jones and others, 1984) is an Upper Jurassic to Lower Cretaceous flysch sequence that consists dominately of graywacke, phyllite and shale with local lenses of conglomerate. Minor limestone, radiolarian chert and ferruginous sandstone, siltstone and tuff are present. The rocks of this terrane are strongly deformed and isoclinally folded.

The Alaska-Aleutian Range batholith includes rocks formed during three periods of igneous activity (Reed and Lanphere, 1973): a Middle to Late Jurassic period, a Late Cretaceous to early Tertiary period, and a middle Tertiary period. Jurassic plutonic rocks have not been identified in the Lime Hills quadrangle. Cretaceous to Tertiary plutonic rocks consist of an older group of quartz diorite to granodiorite plutons and a younger group of quartz monzonite to granite plutons. Middle tertiary igneous activity consists of quartz monzonite to granite plutons and intermediate to felsic volcanic flows, breccias, and tuffs.

The Denali fault system, a major northeast-trending, strike-slip fault system, occurs in the western portion of the quadrangle. On the Farewell segment of this system, $150 \mathrm{~km}$ of right-lateral movement has been interpreted (Blodgett and Clough, 1985). The Denali-Farewell fault system shows evidence of movement as late as Holocene time and may still be active (Bundtzen and others, 1986).

\section{METHODS OF STUDY}

\section{Sample Media}

Analyses of stream sediment represent the chemistry of rock material eroded from a drainage basin upstream from a sample site. Such information is useful in identifying those basins which contain concentrations of elements that may be related to mineral deposits. Whereas the composition of samples of bulk stream sediment is most reflective of lithologies exposed within a drainage basin, heavy-mineral concentrates of stream sediment are selectively enriched in certain minerals, including many which may be ore-related. This 
concentration process permits detection of some elements that are not easily or reliably detected in bulk stream sediment.

\section{Sample Collection}

The sampling for this survey included collection of stream-sediment and heavy-mineral-concentrate samples at most sites. This report concerns the 914 concentrate samples that were collected in the study area (plate 1). Geochemical data for stream-sediment samples is presented in Motooka and others (1989). Results of mineralogical analysis of heavy-mineral-concentrate samples for this project are presented in Allen and Slaughter (1989).

Geological and geochemical data for rock samples collected from the study area are presented in Allen and others (1989).

Sampling density for the drainage survey is about one sample per $4,5 \mathrm{mi}^{2}$ $\left(11.6 \mathrm{~km}^{2}\right)$, The area of drainage basins sampled ranges from 1 to $10 \mathrm{mi}^{2}$ (2.6-26 $\left.\mathrm{km}^{2}\right)$. Stream sediment and concentrate samples are composites of active alluvium collected primarily from first-order (unbranched) or second-order (below the junction of two first-order) streams as shown on the USGS topographic map of plate 1. In areas of active glaciation, samples were collected from major morraines selected as being representative of portions of the basin above.

Heavy-mineral concentrate samples were collected from bulk sediment which was initially sieved through a 10 -mesh $(2.0 \mathrm{~mm})$ screen to remove coarse rock and vegetation fragments. Enough material was collected to fill a 16 in. diameter stainless-steel goldpan. A 1-2 lb $(0.5-1 \mathrm{~kg})$ bulk sediment sample was removed from the pan and was used as the stream-sediment sample. The remaining material was panned until most quartz, feldspar, organic and clay-sized material was removed. The remaining material was packaged as the heavy-mineral-concentrate sample for additional laboratory preparation.

\section{Sample Preparation}

After drying, the heavy-mineral-concentrate samples were sieved through a 20-mesh ( $1 \mathrm{~mm})$ sieve. Bromoform (S.G. 2.85) was used to remove remaining light minerals from the concentrates collected in the field. The resultant heavy-mineral sample was separated into three fractions using an electromagnet. The most magnetic fraction, consisting dominantly of magnetite and ilmenite, and the intermediate magnetic fraction, consisting dominantly of ferromagnesian silicates and oxides, were archived. The nonmagnetic fraction consists dominantly of heavy accessory minerals such as zircon, sphene, and apatite, but also contains most ore minerals when present in a sample. These magnetic separates are the same that would be produced using a Frantz Isodynamic Separator set at a slope of 15 degrees and a tilt of 10 degrees with a current of 0.2 ampere to remove the most magnetic minerals and a current of 0.6 ampere to separate the intermediate and nonmagnetic fractions. The nonmagnetic fraction was split using a riffle splitter, and one part was used for mineralogical analysis, and the other split for chemical analysis. The split for chemical analys is was pulverized (approx. 200 mesh; $63 \mu \mathrm{m})$ by hand using an agate mortar and pestle.

\section{Sample Analysis}

The pulverized nonmagnetic, heavy-mineral-concentrate samples were analysed for 38 elements using semiquantitative, direct-current-arc, emission 
spectrographic methods (Grimes and Marranzino, 1968; Myers and others, 1961). Spectrographic results were determined by visually comparing spectra derived from the sample against spectra obtained from laboratory reference standards. Standard concentrations are geometrically spaced over any given order of magnitude of concentration such that values reported for each sample are reported in the geometric sequence $10,15,20,30,50,70,100$ etc. The elements determined and their limits of determination are listed in table 1. The precision of the Grimes and Marranzino (1968) method is plus or minus one reporting interval at 83 percent, or two intervals at 96 percent confidence (Motooka and Grimes, 1976). The precision of the Myers and others (1961) method is plus or minus one interval at 68 percent, or two intervals at 95 percent confidence. The results of these analyses are presented in table 2.

\section{DATA STORAGE SYSTEM}

Upon completion of the analytical work, the analytical results were entered into a computer-based file as part of the USGS Rock Analys is Storage System (RASS) database. This database contains both descriptive geological information and analytical data. Any of this information may be retrieved and converted to a binary form (STATPAC) for computerized analys is or publication (VanTrump and Miesch, 1976).

\section{DESCRIPTION OF DATA TABLE}

Table 2 contains the analytical results for the nonmagnetic, heavymineral concentrate samples. The data are arranged such that the first column contains the USGS-assigned sample numbers. These numbers correspond to those shown on plate 1, but without the "LH" prefix and "C" suffix. The designation " $s$ " on element heading indicates spectrographic analysis. The letter " $N$ " in the table indicates that an element was looked for but not observed. If an element was observed but was below the lowest reporting value, a "less than" symbol (<) was entered in the table in front of the lower limit of determination. If an element was observed but was above the upper reporting value, a "greater than" symbol ( $>$ ) was entered in the table in front of the upper limit of determination. Because of the formatting used in the computer program that produced table 2, some of the elements listed in this table (Fe, $\mathrm{Mg}, \mathrm{Ca}, \mathrm{Na}, \mathrm{Ti}, \mathrm{P}$, and $\mathrm{Ag}$ ) carry one or more nonsignificant zeros to the right of the significant digits. The analyst did not determine these elements to the accuracy suggested by the extra zeros. Values determined for the major elements, $\mathrm{Fe}, \mathrm{Mg}, \mathrm{Ca}, \mathrm{Na}, \mathrm{Ti}$, and $\mathrm{P}$ are given in weight percent; all others are in parts per million (micrograms/gram). The analyses for palladium were all below the lower limit of determination, and are not included in table 2.

\section{ACKNOWLEDGMENTS}

We would like to acknowledge the following people for the ir assistance in the collection of samples for this study: E. Bailey, B. Cieutat, T. Delaney, K. Duttweiler-Kelley, R. Goldfarb, A. Hofstra, G. Lee, E. Leibold, S. Rose, and S. Smith. The assistance of A. Sutton proved critical in the retrieval of data from the complicated PLUTO analytical database. 


\section{REFERENCES CITED}

Allen, M.S., Malcolm, M.J., Motooka, J.M., and Slaughter, K.E., 1989, Geologic description, chemical analyses, and sample locality map for rock samples collected from the eastern part of the Lime Hills quadrangle, Alaska:

U.S. Geological Survey Open-File Report 90-69, 49 p.

Allen, M.S., and Slaughter, K.E., 1989, Mineralogical data and sample locality map for nonmagnetic, heavy-mineral-concentrate samples collected from the eastern part of the Lime Hills quadrangle, Alaska: U.S. Geological Survey Open-File Report 90-67, $62 \mathrm{p}$.

Blodgett, R.B., and Clough, J.G., 1985, The Nixon Fork Terrane--Part of an in situ peninsular extension of the Paleozoic North American continent [abs]: Geological Society of America Abstracts with Programs, v. 17, no. 6 , p. 342 .

Bundtzen, T.K., Kline, J.T., Clautice, K.H., and Adams, D.D., 1986, Minerals potentia1, Department of Natural Resources Kuskokwim planning block, Alaska: Alaska Division of Geological and Geophysical Surveys Public-Data File 86-53e, 44 p.

Bundtzen, T.K., Kline, J.T., Smith, T.E., and Albanese, M.D., 1987, Geologic map of the McGrath A-2 quadrangle, Alaska: Alaska Division of Geological and Geophysical Surveys Professional Report 91, scale 1:63,360.

Cady, W.M., Wallace, R.E., Hoare, J.M., and Webber, E.J., 1955, The central Kuskokwim region, Alaska: U.S. Geological Survey Professional Report 268, $132 \mathrm{p}$.

Churkin, Michael, Jr., 1984, Nixon Fork-Dillinger terranes--a dismembered Paleozoic craton margin in Alaska displaced from Yukon Territory [abs]: Geological Society of America Abstracts with Programs, v. 16, no. 5, p. 275.

Grimes, D.J., and Marranzino, A.P., 1968, Direct-current arc and alternating current spark emission spectrograghic field methods for the semiquantitative analysis of geologic materials: U.S. Geological Survey Circular $591,6 \mathrm{pp}$.

Jones, D.L., Silberling, N.J., Coney, P.J., and Plafker, George, 1984, Lithotectonic terrane map of Alaska west of the 141 st meridian, in Silberling, N.J, and Jones, D.L, [eds], Lithotectonic terrane maps of the North America Cordillera: U.S. Geological Survey Open-File Report 84-523, A1-A12.

Motooka, J.M., Allen, M.S., Malcolm, M.J., and Slaughter, K.E., 1989 , Analytical results and sample locality map for stream-sediment samples collected from the eastern part of the Lime Hills quadrangle, Alaska: U.S. Geological Survey Open-File Report 90-70, $103 \mathrm{p}$.

Motooka, J.M., and Grimes, D.J., 1976, Analytical precision of one-sixth order semiquatitative spectrographic analysis: U. S. Geological Survey Circular 738, $25 \mathrm{p}$.

Myers, A.T., Havens, R.G., and Dunton, P.J., 1961, A spectrochemical method for the semiquantitative analyses of rocks, minerals, and ores: U.S. Geological Survey Bulletin 1084-I, p. 1207-1229.

Reed, B.L., and Lanphere, M.A., 1973, Alaska-Aleut ian Range batholith-geochronology, chemistry, and relation to circum-Pacific plutonism: Geological Society of America Bulletin, v. 84, p. 2583-2610.

Reed, B.L., and Nelson, S.W., 1980, Geologic map of the Talkeetna quadrangle, Alaska: U.S. Geological Survey Investigations Map I-1174, scale $1: 250,000$.

VanTrump, George, Jr., and Miesch A.T., 1976, The U.S. Geological Survey RASS-STATPAC system for management and statistical reduction of geochemical data: Computers and Geosciences, v. 3, p. 475-488. 
TABLE 1.--Limits of determination for the spectrographic analysis of heavy-mineral concentrate samples, based on 5-mg sample

[The values shown are the limits of determination assigned by the Grimes and Marranzino (1968) method, except for uranium values which are assigned by Myers and others (1961)]

\begin{tabular}{llc}
\hline & Lower determination limit & Upper determination limit \\
& & \\
& & 50 \\
Iron (Fe) & 0.1 & 20 \\
Magnesium (Mg) & 0.05 & 50 \\
Calcium (Ca) & 0.1 & 10 \\
Sodium (Na) & 0.5 & 2 \\
Titanium (Ti) & 0.005 & 20 \\
Phosphorus (P) & 0.5 &
\end{tabular}

Parts per million

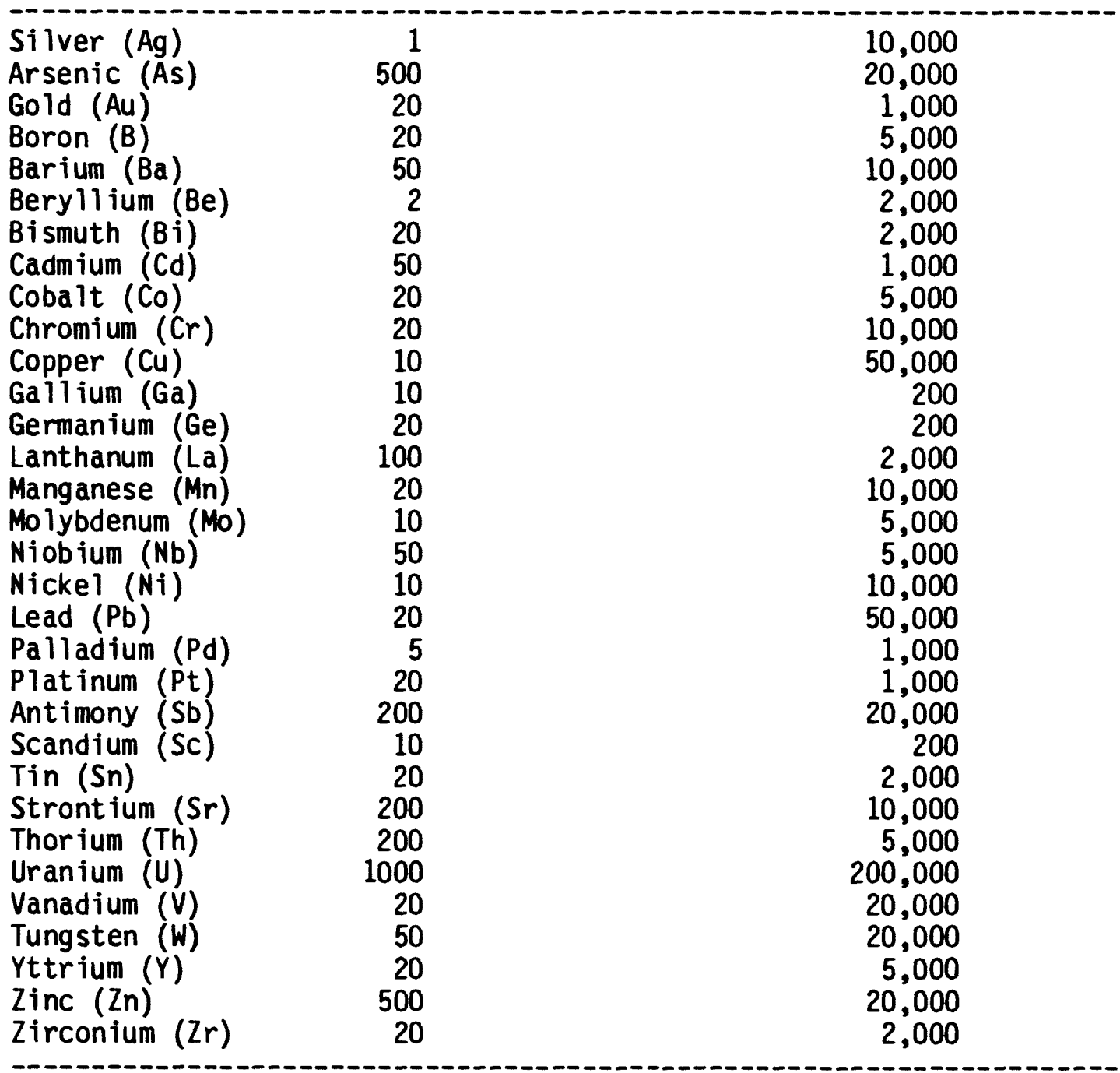


tABLE 2. GEOCHEMICAL DATA FOR NONMAGNETIC, heAVY-MINERAL-CONCENTRATE SAMPLES, Lime hills QUAdRANGLE, ALASKa

[N, not detected; <, detected but below the limit of determination shown; >, determined to be greater than the value shown.]

Sample Latitude Longitude fe $\%$-s $M g$ \%-s $\quad$ Ca $\%$-s $\quad \mathrm{Na} \%$-s $\quad$ Ti $\%$-s $\quad$ P $\%$-s Ag ppm-s As ppm-s

\begin{tabular}{|c|c|c|c|c|c|c|c|c|c|c|c|c|c|}
\hline LH0001C & 6141 & 26 & 153 & 36 & 46 & 1.00 & .10 & 1.00 & $<.5$ & 1.000 & $N$ & $N$ & N \\
\hline LH0003C & 6142 & 58 & 153 & 44 & 18 & 2.00 & $<.05$ & .70 & .5 & .200 & .7 & 5.0 & 1,500 \\
\hline LH0004C & 6132 & 11 & 153 & 27 & 4 & 5.00 & .30 & 3.00 & .5 & $>2.000$ & 3.0 & 5.0 & 700 \\
\hline LH0005C & 6129 & 41 & 153 & 29 & 23 & 5.00 & .15 & 10.00 & .7 & $>2.000$ & 5.0 & 200.0 & 7,000 \\
\hline LH0006C & 6130 & 5 & 153 & 29 & 48 & 2.00 & 1.50 & 1.50 & .7 & $>2.000$ & 1.0 & 7.0 & 500 \\
\hline LHO007C & 6129 & 59 & 153 & 233 & 33 & 3.00 & .10 & 20.00 & 1.0 & .300 & 7.0 & 10.0 & 5,000 \\
\hline LHOOOBC & 6128 & 23 & 153 & 23 & 8 & 1.00 & $<.05$ & 15.00 & .7 & $>2.000$ & 5.0 & 5.0 & 700 \\
\hline LH0009C & 6131 & 59 & 153 & 215 & 54 & .50 & $<.05$ & 20.00 & .7 & 2.000 & 15.0 & 10.0 & N \\
\hline LHOO10C & 6130 & 32 & 153 & 18 & 22 & 5.00 & .20 & 10.00 & 1.0 & $>2.000$ & 1.5 & 15.0 & 1,500 \\
\hline LHO011C & 6131 & 41 & 153 & 18 & 0 & 3.00 & $<.05$ & 5.00 & 3.0 & .070 & .7 & 15.0 & 7,000 \\
\hline LHO012C & 6134 & 21 & 153 & 23 & 28 & 1.00 & .07 & 7.00 & .5 & $>2.000$ & 5.0 & 700.0 & 2,000 \\
\hline LH0013C & 6137 & 51 & 153 & 22 & 16 & 7.00 & .07 & 20.00 & .5 & .700 & 10.0 & 500.0 & 2,000 \\
\hline LH0014C & 6136 & 22 & 153 & 23 & 9 & 3.00 & .07 & 30.00 & .5 & .300 & 10.0 & 50.0 & $>20,000$ \\
\hline LH0015C & 6141 & 31 & 153 & 13 & 43 & 5.00 & .07 & 15.00 & 1.0 & 1.500 & 2.0 & $2,000.0$ & 7,000 \\
\hline LH0016C & 6159 & 52 & 153 & 48 & 22 & 2.00 & .20 & 10.00 & .7 & .050 & $<.5$ & 150.0 & 3,000 \\
\hline LH0017C & 6158 & 31 & 153 & 46 & 20 & 2.00 & .20 & 15.00 & .5 & .700 & 3.0 & 20.0 & N \\
\hline LH0018C & 6159 & 14 & 153 & 44 & 10 & 7.00 & .15 & 15.00 & .5 & 1.500 & 7.0 & 30.0 & 20,000 \\
\hline LH0019C & 6159 & 21 & 153 & 40 & 41 & 2.00 & .30 & 20.00 & .7 & 1.500 & 10.0 & 2.0 & 1,500 \\
\hline LH0020C & 6158 & 23 & 153 & 37 & 56 & 1.00 & .15 & 2.00 & $<.5$ & .300 & 1.5 & 7.0 & N \\
\hline LH0021C & 6159 & 56 & 153 & $32 ?$ & 37 & .50 & .10 & 5.00 & $N$ & 2.000 & 5.0 & $N$ & N \\
\hline LH0022C & 6154 & 36 & 153 & 42 & 6 & .20 & $<.05$ & 3.00 & 1.0 & .300 & .5 & $N$ & $<500$ \\
\hline LH0023C & 6152 & 26 & 153 & 413 & 30 & 15.00 & $<.05$ & 1.50 & $N$ & 1.000 & .7 & 100.0 & $>20,000$ \\
\hline LH0024C & 6152 & 25 & 153 & 445 & 54 & 20.00 & .05 & 1.00 & .7 & .150 & 1.0 & 70.0 & $>20,000$ \\
\hline LH0025C & 6152 & 33 & 153 & 44 & 47 & 20.00 & .05 & 1.00 & .7 & .070 & 1.0 & 100.0 & $>20,000$ \\
\hline LH0026C & 6154 & 23 & 153 & 323 & 37 & 1.50 & .30 & 5.00 & .7 & 2.000 & 3.0 & 100.0 & 15,000 \\
\hline LH0027C & 6152 & 13 & 153 & 33 & 3 & 7.00 & .15 & 2.00 & .3 & $>2.000$ & 2.0 & 50.0 & $>20,000$ \\
\hline LHOO2BC & 6150 & 21 & 153 & 37 & 50 & 2.00 & .15 & 10.00 & 1.5 & 2.000 & 3.0 & 30.0 & 3,000 \\
\hline LH0029C & 6145 & 48 & 153 & 38 & 47 & 1.50 & .07 & 7.00 & .7 & 1.000 & 2.0 & 200.0 & 15,000 \\
\hline LH0030C & 6146 & 55 & 153 & 43 & 3 & 10.00 & .10 & 3.00 & 1.0 & 1.000 & 1.0 & 100.0 & $>20,000$ \\
\hline LHO03IC & 6147 & 57 & 153 & 443 & 33 & 2.00 & .30 & 20.00 & 1.0 & $>2.000$ & 5.0 & 50.0 & 15,000 \\
\hline LH0032C & 6148 & 2 & 153 & 49 & 57 & .50 & $<.05$ & 2.00 & 1.0 & .500 & .5 & 100.0 & 5,000 \\
\hline LH0033C & 6145 & 47 & 153 & 47 & 49 & .30 & $<.05$ & 1.00 & 1.5 & .070 & .7 & N & N \\
\hline LH0034C & 6149 & 50 & 153 & 343 & 38 & 15.00 & .70 & 7.00 & .7 & $>2.000$ & 3.0 & 100.0 & $>20,000$ \\
\hline LH0035C & 6145 & 6 & 153 & 32 & 29 & 2.00 & .15 & 10.00 & .5 & $>2.000$ & 5.0 & 10.0 & $<500$ \\
\hline LH0036C & 6153 & 22 & 153 & 263 & 39 & 2.00 & .15 & 7.00 & .5 & 1.500 & 3.0 & 5.0 & 7,000 \\
\hline LH0037C & 6150 & 2 & 153 & 183 & 35 & .70 & .10 & 10.00 & 2.0 & .500 & 3.0 & $N$ & $N$ \\
\hline LH0038C & 6153 & 0 & 153 & 193 & 36 & 2.00 & .07 & 10.00 & 1.0 & .300 & 3.0 & $N$ & $N$ \\
\hline LH0039C & 6155 & 42 & 153 & 11 & 49 & 1.00 & .15 & 10.00 & .7 & $>2.000$ & 7.0 & $N$ & N \\
\hline LHOO4OC & 6154 & 53 & 153 & 24 & 54 & 5.00 & .20 & 5.00 & .7 & .500 & .7 & 30.0 & 7,000 \\
\hline LH0041C & 6159 & 44 & 153 & 20 & 51 & .50 & .10 & 15.00 & 1.0 & .500 & 5.0 & $N$ & N \\
\hline LH0042C & 6159 & 19 & 153 & 15 & 58 & 1.50 & .07 & 10.00 & .5 & 2.000 & 3.0 & $N$ & 700 \\
\hline LH0043C & 6159 & 10 & 153 & 23 & 49 & .50 & .07 & 30.00 & 1.5 & .200 & 20.0 & $N$ & $N$ \\
\hline LH0044C & 6159 & 13 & 153 & 23 & 58 & .50 & .07 & 30.00 & 1.0 & .300 & 20.0 & $N$ & N \\
\hline LH0045C & 6158 & 37 & 153 & 26 & 46 & .50 & .07 & 30.00 & 1.0 & .200 & 20.0 & $N$ & $N$ \\
\hline LH0046C & 6146 & 1 & 153 & 22 & 47 & 7.00 & .30 & 10.00 & .7 & 1.000 & 5.0 & 5.0 & N \\
\hline LH0047C & 6141 & 30 & 153 & 27 & 21 & 7.00 & .20 & 3.00 & .7 & $>2.000$ & 3.0 & 200.0 & 3,000 \\
\hline LH0048C & 6135 & 1 & 153 & 27 & 24 & .50 & .07 & 2.00 & .7 & $>2.000$ & 3.0 & N & N \\
\hline LH0049C & 6128 & 45 & 153 & 1 & 2 & 10.00 & .15 & 5.00 & 1.5 & 1.500 & 3.0 & 200.0 & 3,000 \\
\hline LH0050C & 6128 & 50 & 153 & 0 & 43 & .70 & .07 & 30.00 & 1.5 & $>2.000$ & 3.0 & 5.0 & 500 \\
\hline LH0051C & 6120 & 19 & 153 & 1 & 42 & .30 & $<.05$ & 1.50 & 1.5 & .150 & 1.0 & 20.0 & 1,000 \\
\hline
\end{tabular}


TABLE 2. GEOCHEMICAL DATA FOR NONMAGNETIC, HEAVY-MINERAL-CONCENTRATE SAMPLES, LIME HILLS QUADRANGLE, ALASKA--CONT InUEd

Sample Au ppm-s B ppm-s Ba ppm-s Be ppm-s Bi ppm-s Co ppm-s Co ppm-s Cr ppm-s Cu ppm-s

\begin{tabular}{|c|c|c|c|c|c|c|c|c|c|}
\hline LH0001C & $N$ & 30 & 7,000 & $N$ & $N$ & N & $N$ & 150 & 15 \\
\hline LHOOO3C & N & 20 & 3,000 & N & 150 & $N$ & $<20$ & $N$ & 20 \\
\hline LHOOOLC & $N$ & 70 & 5,000 & 3 & N & $N$ & 50 & 300 & 150 \\
\hline LH0005C & 200 & 50 & 7,000 & 2 & 300 & N & 30 & 50 & 100 \\
\hline LH0006C & $N$ & $>5,000$ & 700 & 3 & 1,500 & $N$ & $N$ & 500 & 30 \\
\hline LH0007C & $N$ & 200 & 700 & $N$ & N & $N$ & 70 & $<20$ & 30 \\
\hline LH0008C & N & $<20$ & 7,000 & 5 & $N$ & $N$ & 20 & $<20$ & 10 \\
\hline LHOOO9C & $N$ & N & $>10,000$ & N & 30 & N & $N$ & 70 & 20 \\
\hline LHOO10C & $N$ & 20 & 700 & 3 & 500 & $\mathbf{N}$ & 200 & 30 & 200 \\
\hline LH0O11C & N & $\mathbf{N}$ & $>10,000$ & $N$ & 500 & $N$ & 70 & $<20$ & 30 \\
\hline LH0012C & 30 & N & $>10,000$ & $N$ & 2,000 & $N$ & $<20$ & 300 & 20 \\
\hline LH0013C & N & N & $>10,000$ & $N$ & 700 & $N$ & 70 & 30 & 200 \\
\hline LH0014C & N & N & $>10,000$ & $N$ & 150 & $N$ & 100 & $<20$ & 50 \\
\hline LH0015C & 70 & $N$ & $>10,000$ & 10 & 300 & $N$ & $<20$ & $<20$ & 100 \\
\hline LH0016C & $\mathbf{N}$ & 70 & $>10,000$ & 15 & 70 & 50 & $<20$ & $<20$ & 150 \\
\hline LH0017C & N & 20 & $>10,000$ & $N$ & $<20$ & $N$ & $<20$ & 20 & 30 \\
\hline LH0018C & N & 100 & $>10,000$ & $N$ & $<20$ & $N$ & 100 & 20 & 70 \\
\hline LH0019C & $N$ & 50 & $>10,000$ & $N$ & $\mathbf{N}$ & $N$ & 20 & 70 & 50 \\
\hline LH002OC & $N$ & 20 & $>10,000$ & $N$ & $N$ & N & $<20$ & $<20$ & 30 \\
\hline LHOOZ21C & N & 500 & 2,000 & 5 & N & N & 0 & 50 & 20 \\
\hline LH0022C & $N$ & N & 500 & 3 & N & N & N & $<20$ & 10 \\
\hline LH0023C & N & N & 100 & 2 & 700 & N & 2,000 & $<20$ & 150 \\
\hline LH0024C & N & $N$ & 150 & $\mathbf{N}$ & 200 & $N$ & 700 & $<20$ & 150 \\
\hline LH0025C & N & N & 150 & $\mathbf{N}$ & 200 & N & 700 & $<20$ & 150 \\
\hline LHOO26C & 100 & 1,000 & 3,000 & 3 & 2,000 & $N$ & 200 & 150 & 20 \\
\hline LH0027C & 70 & N & $>10,000$ & N & 50 & $N$ & 70 & $<20$ & 100 \\
\hline LH0028C & N & 50 & 2,000 & 3 & 700 & N & 200 & 70 & 50 \\
\hline LH0029C & 1,000 & $<20$ & $>10,000$ & N & $N$ & N & 30 & 30 & 15 \\
\hline LH0030C & 150 & 50 & 3,000 & $N$ & 20 & N & 300 & 30 & 50 \\
\hline LH0031C & 150 & 700 & 1,000 & 2 & 20 & N & 150 & 30 & 150 \\
\hline LH0032C & $N$ & N & 200 & 3 & 200 & N & N & N & 15 \\
\hline LH0033C & N & 70 & 2,000 & 3 & N & $N$ & $N$ & $<20$ & $<10$ \\
\hline LH0034C & N & 100 & $>10,000$ & $N$ & 30 & $\mathbf{N}$ & 100 & 200 & 2,000 \\
\hline LH0035C & N & 50 & $>10,000$ & N & N & $\mathbf{N}$ & 20 & 200 & 70 \\
\hline LH0036C & N & 70 & $>10,000$ & N & 300 & $N$ & 200 & 50 & 50 \\
\hline LH0037C & N & 20 & 1,000 & N & N & N & N & 20 & 20 \\
\hline LH0038C & N & $N$ & $>10,000$ & 3 & N & N & N & $<20$ & $<10$ \\
\hline LH0039C & N & 50 & $>10,000$ & N & N & N & $<20$ & 100 & 50 \\
\hline LH004OC & 70 & 30 & $>10,000$ & N & 70 & N & 300 & 20 & 70 \\
\hline LH0O41C & $\mathbf{N}$ & N & $>10,000$ & $N$ & $N$ & N & $<20$ & $<20$ & $<10$ \\
\hline LH0042C & N & N & $>10,000$ & $N$ & N & N & $<20$ & 30 & 150 \\
\hline LH0043C & $N$ & N & 300 & N & N & N & $N$ & $<20$ & $<10$ \\
\hline LHOO44C & $N$ & N & 150 & $\mathbf{N}$ & N & N & N & $<20$ & $<10$ \\
\hline LH0045C & $N$ & N & 150 & N & N & $N$ & $\mathbf{N}$ & $<20$ & 10 \\
\hline LH0046C & $N$ & 150 & $>10,000$ & $N$ & 1,000 & N & 30 & 70 & 700 \\
\hline LH0O47C & $<20$ & $N$ & $>10,000$ & 2 & $<20$ & $N$ & 30 & 50 & 500 \\
\hline LH0O48C & N & 20 & $>10,000$ & N & N & N & $<20$ & 30 & 10 \\
\hline LH0049C & $N$ & $N$ & $>10,000$ & $N$ & 150 & N & 20 & $<20$ & 150 \\
\hline LHOOSOC & $N$ & $\mathbf{N}$ & 150 & 3 & 30 & $\mathbf{N}$ & $\mathbf{N}$ & $<20$ & 10 \\
\hline LHO0S1C & $\mathbf{N}$ & $\mathbf{N}$ & 500 & 3 & 300 & $N$ & 30 & $<20$ & $<10$ \\
\hline
\end{tabular}




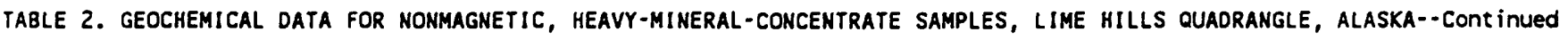

Sample Ga ppm-s Ge ppm-s La ppm-s Mn ppm-s Mo ppm-s Nb ppm-s Ni ppm-s Pb ppm-s Pt ppm-s Sb ppm-s

\begin{tabular}{|c|c|c|c|c|c|c|c|c|c|c|}
\hline LH0001C & 30 & N & 100 & 150 & $N$ & 50 & $<10$ & N & $N$ & $N$ \\
\hline LH0003C & $N$ & N & N & 70 & 70 & $<50$ & $<10$ & 200 & N & $N$ \\
\hline LH0004C & 10 & $N$ & 150 & 700 & 15 & 200 & 50 & 70 & $N$ & N \\
\hline LH0005C & 15 & $N$ & 200 & 1,000 & $N$ & 70 & 10 & 10,000 & $N$ & $<200$ \\
\hline LH0006C & 20 & N & 100 & 200 & $N$ & 100 & 10 & 200 & N & $<200$ \\
\hline LH0007C & 10 & $N$ & 1,000 & 300 & $N$ & $<50$ & 10 & 1,000 & N & N \\
\hline LH0008C & $<10$ & $\mathbf{N}$ & 500 & 200 & $N$ & 50 & $<10$ & 700 & N & 300 \\
\hline LH0009C & 10 & N & 300 & 700 & $N$ & 70 & $<10$ & 7,000 & N & N \\
\hline LH0010C & 30 & $<20$ & 150 & 1,500 & 15 & 50 & $<10$ & 700 & N & N \\
\hline LHO011C & 15 & $N$ & $N$ & 150 & 100 & $<50$ & 10 & 3,000 & N & $N$ \\
\hline LH0012C & N & $N$ & 100 & 200 & $N$ & 150 & 10 & 5,000 & N & $N$ \\
\hline LH0013C & N & N & 700 & 300 & 150 & $<50$ & 50 & $>50,000$ & N & $N$ \\
\hline LH0014C & $N$ & N & 700 & 500 & N & $<50$ & 20 & 7,000 & N & $N$ \\
\hline LH0015C & $<10$ & N & 200 & 300 & 1,500 & 50 & $<10$ & $>50,000$ & N & N \\
\hline LH0016C & $<10$ & N & 500 & 100 & 70 & $<50$ & N & 30,000 & N & 300 \\
\hline LH0017C & $<10$ & N & N & 150 & $N$ & $<50$ & 20 & 3,000 & $N$ & N \\
\hline LH0018C & N & N & $N$ & 100 & N & $<50$ & 200 & 7,000 & $N$ & $<200$ \\
\hline LH0019C & $<10$ & N & N & 200 & N & $<50$ & 30 & 500 & $N$ & N \\
\hline LH0020C & N & N & 150 & 150 & N & $<50$ & 10 & 1,000 & $N$ & $N$ \\
\hline LH0021C & N & N & 300 & 150 & 50 & $<50$ & $N$ & 150 & $N$ & $N$ \\
\hline LH0022C & $<10$ & $N$ & N & 70 & 150 & $<50$ & N & 700 & $N$ & N \\
\hline LH0023C & N & $N$ & N & 50 & 300 & 70 & 1,000 & 5,000 & $N$ & $N$ \\
\hline LHOO24C & $<10$ & $N$ & $N$ & 70 & 150 & $<50$ & 300 & 3,000 & N & N \\
\hline LH0025C & $<10$ & $N$ & N & 70 & 50 & $<50$ & 200 & 3,000 & N & $<200$ \\
\hline LHOO26C & 10 & N & 200 & 500 & N & 100 & 300 & 500 & $N$ & $<200$ \\
\hline LH0027C & N & N & $N$ & 150 & $N$ & 70 & 20 & 2,000 & $N$ & 300 \\
\hline LH0028C & 15 & N & 300 & 500 & 30 & 100 & 20 & 300 & $N$ & N \\
\hline LHO029C & $<10$ & N & 100 & 150 & 50 & 100 & 30 & 1,500 & $N$ & 1,000 \\
\hline LH0030C & $<10$ & N & 150 & 100 & 100 & 150 & 500 & 2,000 & N & $<200$ \\
\hline LH0031C & $<10$ & N & 150 & 1,000 & $N$ & 150 & 150 & 1,000 & $N$ & N \\
\hline LH0032C & $<10$ & N & N & 100 & 300 & 50 & $N$ & 1,000 & N & $N$ \\
\hline LH0033C & $<10$ & $N$ & N & 100 & 30 & $<50$ & N & 70 & N & N \\
\hline LH0034C & 10 & N & 300 & 500 & 20 & $<50$ & 100 & 7,000 & N & $<200$ \\
\hline LH0035C & $<10$ & $<20$ & 300 & 300 & N & 100 & 20 & 200 & $N$ & 300 \\
\hline LH0036C & 10 & $<20$ & 150 & 300 & 30 & 50 & 100 & 50 & $N$ & N \\
\hline LH0037C & 15 & N & 200 & 300 & $N$ & $<50$ & $<10$ & 20 & $N$ & N \\
\hline LH0038C & 15 & $N$ & 150 & 700 & $N$ & $<50$ & N & 70 & $N$ & $N$ \\
\hline LH0039C & 10 & $N$ & 150 & 500 & $N$ & 50 & 20 & 300 & $N$ & N \\
\hline LH0040C & $<10$ & $N$ & 150 & 200 & 70 & 100 & 300 & 70 & N & N \\
\hline LH004IC & $<10$ & $N$ & 500 & 150 & $N$ & $<50$ & $<10$ & $N$ & $N$ & $N$ \\
\hline LH0042C & $<10$ & $N$ & 200 & 200 & N & $<50$ & 10 & 700 & $N$ & $N$ \\
\hline LH0043C & $<10$ & $N$ & 1,500 & 300 & N & $<50$ & $<10$ & N & $N$ & $N$ \\
\hline LHOO44C & $<10$ & N & 1,500 & 300 & N & $<50$ & $<10$ & N & $N$ & $N$ \\
\hline LH0045C & $<10$ & N & 1,500 & 300 & N & $<50$ & $<10$ & 700 & $N$ & $N$ \\
\hline LH0046C & 30 & N & 300 & 500 & N & $<50$ & 30 & 30 & N & $N$ \\
\hline LH0047C & 15 & $N$ & 150 & 150 & N & 70 & 30 & 1,500 & N & N \\
\hline LH0048C & $<10$ & $N$ & 150 & 150 & N & 200 & 10 & 20 & N & 300 \\
\hline LHOO49C & 10 & $N$ & 300 & 500 & 100 & 50 & 10 & 5,000 & N & N \\
\hline LH0050C & 15 & $N$ & 700 & 500 & 30 & 150 & $<10$ & 300 & $\mathbf{N}$ & N \\
\hline LH0051C & 10 & $N$ & 100 & 150 & 100 & $<50$ & $<10$ & 500 & $N$ & N \\
\hline
\end{tabular}




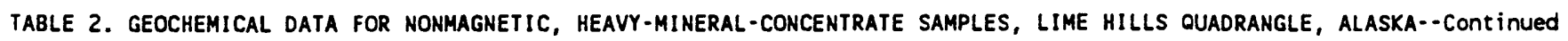

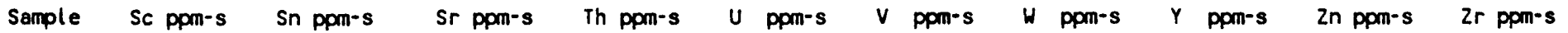

\begin{tabular}{|c|c|c|c|c|c|c|c|c|c|c|}
\hline LHOOOIC & $<10$ & $N$ & $<200$ & N & N & 200 & 100 & 150 & $N$ & $>2,000$ \\
\hline LH0003C & 50 & 150 & N & 2,000 & 2,000 & $N$ & 500 & 1,000 & $N$ & $>2,000$ \\
\hline LH0004C & 100 & 100 & 1,500 & N & N & 700 & 10,000 & 200 & $\mathbf{N}$ & $>2,000$ \\
\hline LHOOO5C & 30 & 50 & 300 & $N$ & $N$ & 150 & 3,000 & 200 & N & $>2,000$ \\
\hline LH0006C & 100 & 1,000 & 200 & $N$ & $N$ & 700 & 500 & 200 & $N$ & $>2,000$ \\
\hline LHO007C & 30 & N & 300 & 200 & $N$ & 20 & 300 & 700 & $N$ & $>2,000$ \\
\hline LHOOOBC & 70 & 150 & 200 & $N$ & $N$ & 70 & 150 & 500 & 1,000 & $>2,000$ \\
\hline LHOOO9C & 20 & $N$ & 700 & $N$ & $N$ & 70 & 2,000 & 300 & N & $>2,000$ \\
\hline LH0010C & 70 & 500 & 300 & $N$ & N & 100 & 200 & 500 & N & $>2,000$ \\
\hline LH0011C & 15 & 2,000 & 200 & N & $N$ & $N$ & 150 & 300 & N & $>2,000$ \\
\hline LH0012C & 70 & 30 & 1,000 & $\mathbf{N}$ & $N$ & 300 & $>20,000$ & 150 & N & $>2,000$ \\
\hline LH0013C & 30 & 2,000 & 700 & $N$ & $N$ & 70 & 700 & 500 & N & $>2,000$ \\
\hline LHOO14C & 20 & 1,500 & 700 & 200 & $N$ & 20 & 200 & 500 & $N$ & $>2,000$ \\
\hline LH0015C & 30 & $>2,000$ & 300 & N & N & 20 & 300 & 500 & 700 & $>2,000$ \\
\hline LH0016C & N & 1,500 & 300 & 700 & $<1,000$ & N & 100 & 1,500 & 3,000 & $>2,000$ \\
\hline LH0017C & 10 & N & 5,000 & N & N & 30 & $N$ & 200 & 1,500 & $>2,000$ \\
\hline LH0018C & 10 & N & 3,000 & N & $N$ & 30 & 200 & 200 & 700 & $>2,000$ \\
\hline LH0019C & 15 & 300 & 3,000 & $<200$ & N & 70 & 70 & 300 & 1,500 & $>2,000$ \\
\hline LH0020C & $N$ & 500 & 7,000 & 700 & $<1,000$ & $<20$ & 50 & 200 & N & $>2,000$ \\
\hline LH0021C & 70 & $>2,000$ & N & $>5,000$ & 7,000 & 70 & 1,000 & 1,500 & N & $>2,000$ \\
\hline LH0022C & 50 & $>2,000$ & $N$ & $>5,000$ & 7,000 & N & 300 & 1,500 & N & $>2,000$ \\
\hline LH0023C & 20 & $>2,000$ & N & $>5,000$ & 5,000 & $N$ & $>20,000$ & 700 & N & $>2,000$ \\
\hline LHO024C & 15 & 500 & N & 500 & N & N & 300 & 300 & N & $>2,000$ \\
\hline LH0025C & 15 & 700 & N & 500 & N & N & 300 & 300 & N & $>2,000$ \\
\hline LHO026C & 50 & $>2,000$ & 200 & 3,000 & 2,000 & 150 & 10,000 & 700 & N & $>2,000$ \\
\hline LH0027C & 20 & 150 & 1,000 & 300 & N & 70 & 500 & 200 & N & $>2,000$ \\
\hline LH0028C & 50 & $>2,000$ & 200 & 700 & 1,000 & 70 & $>20,000$ & 500 & $N$ & $>2,000$ \\
\hline LH0029C & 10 & 20 & 3,000 & N & N & 30 & $>20,000$ & 200 & $N$ & $>2,000$ \\
\hline LHOO3OC & $<10$ & N & 200 & N & $N$ & 20 & $>20,000$ & 150 & $N$ & $>2,000$ \\
\hline LH0031C & 30 & $>2,000$ & 500 & 300 & $N$ & 70 & $>20,000$ & 300 & $N$ & $>2,000$ \\
\hline LHO032C & 70 & $>2,000$ & N & $>5,000$ & 5,000 & N & 1,500 & 1,500 & $N$ & $>2,000$ \\
\hline LHO033C & 100 & 150 & $<200$ & 1,500 & 3,000 & N & 700 & 1,500 & $N$ & $>2,000$ \\
\hline LH0034C & 20 & 1,500 & 700 & N & N & 70 & 200 & 200 & N & $>2,000$ \\
\hline LH0035C & 30 & N & 2,000 & N & $N$ & 200 & 50 & 300 & 2,000 & $>2,000$ \\
\hline LH0036C & 20 & 200 & 1,500 & 1,500 & 1,000 & 70 & 15,000 & 300 & N & $>2,000$ \\
\hline LH0037C & 10 & $\mathbf{N}$ & 700 & N & N & 20 & 50 & 200 & $N$ & $>2,000$ \\
\hline LHOO38C & 50 & 300 & 200 & N & N & $<20$ & 100 & 500 & N & $>2,000$ \\
\hline LH0039C & 20 & N & 3,000 & N & N & 100 & N & 300 & $\mathbf{N}$ & $>2,000$ \\
\hline LHOO $40 \mathrm{C}$ & 15 & 200 & 2,000 & 500 & N & 20 & $>20,000$ & 300 & N & $>2,000$ \\
\hline LHOO41C & 10 & 150 & 1,500 & N & N & 20 & 300 & 500 & N & $>2,000$ \\
\hline LH0042C & 20 & N & 2,000 & $\mathbf{N}$ & $N$ & 70 & N & 200 & $N$ & $>2,000$ \\
\hline LH0043C & 20 & 300 & 300 & N & N & 70 & 300 & 1,000 & $\mathbf{N}$ & $>2,000$ \\
\hline LHO044C & 30 & 300 & 500 & $N$ & $N$ & 70 & 150 & 1,000 & $N$ & $>2,000$ \\
\hline LHO045C & 15 & N & 300 & $N$ & $N$ & 100 & N & 700 & $\mathbf{N}$ & $>2,000$ \\
\hline LHOO $46 \mathrm{C}$ & 15 & $>2,000$ & 700 & N & N & 150 & 300 & 150 & 1,500 & $>2,000$ \\
\hline LH0047C & 30 & $>2,000$ & 500 & $N$ & N & 150 & 2,000 & 100 & 700 & $>2,000$ \\
\hline LH0048C & 70 & 150 & 500 & N & N & 100 & 50 & 150 & N & $>2,000$ \\
\hline LHOO49C & 30 & $>2,000$ & 300 & $\mathbf{N}$ & N & 20 & 5,000 & 200 & 1,000 & $>2,000$ \\
\hline LHOOSOC & 70 & 2,000 & 200 & 500 & $<1,000$ & $<20$ & 100 & 1,500 & N & $>2,000$ \\
\hline LH0051C & 70 & 1,500 & $<200$ & 1,500 & 3,000 & $<20$ & 500 & 1,500 & $\mathbf{N}$ & $>2,000$ \\
\hline
\end{tabular}


TABLE 2. GEOCHEMICAL DATA FOR NONMAGNETIC, hEAVY-MINERAL-CONCENTRATE SAMPLES, LIME HILLS QUAORANGLE, ALASKA--CONT InUEd

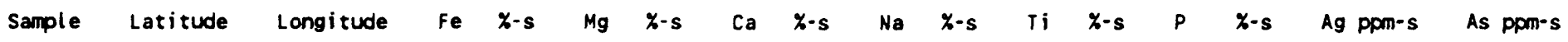

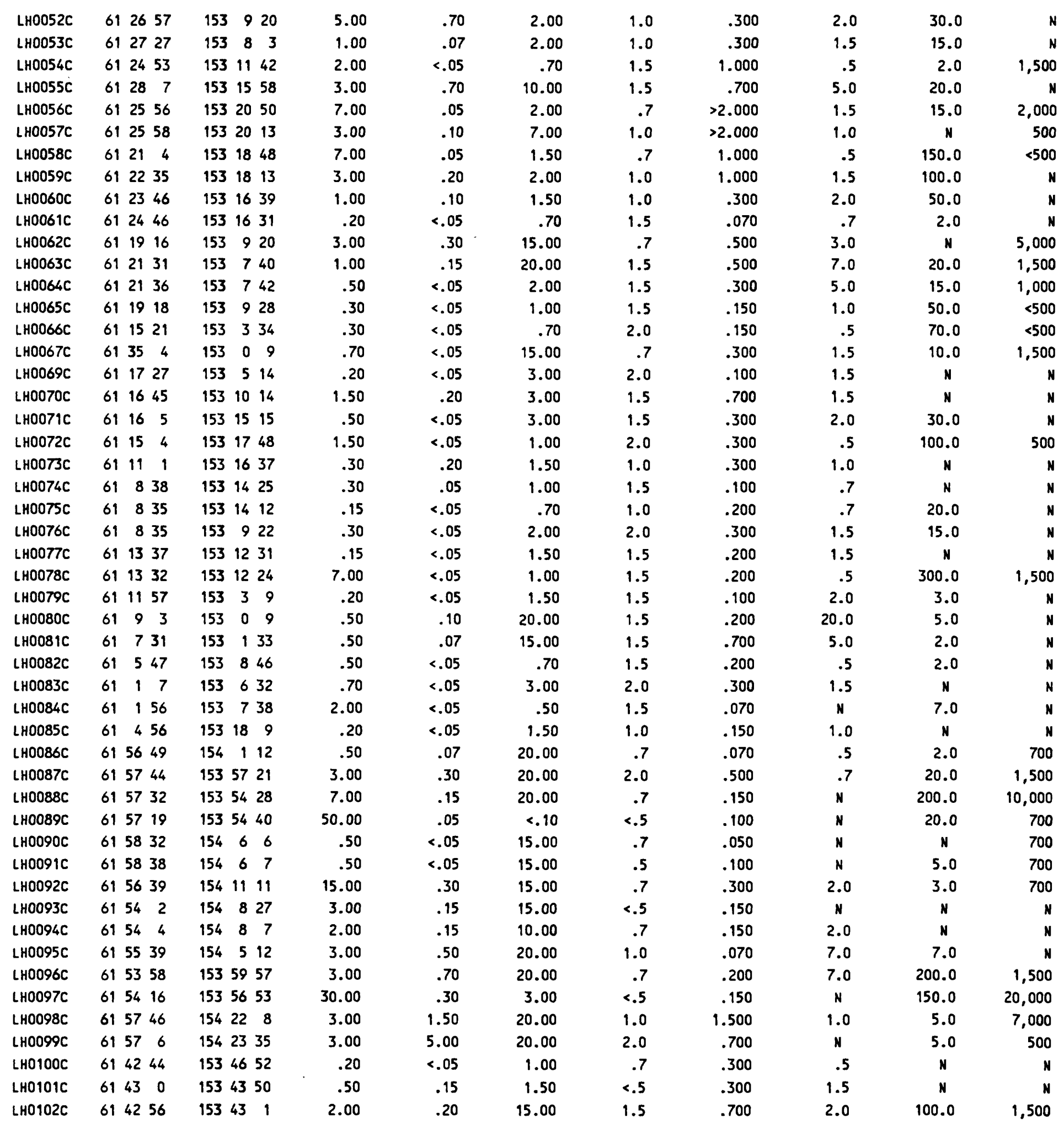


TABLE 2. GEOCHEMICAL DATA FOR NONMAGNETIC, heAVY-MINERAL-CONCENTRATE SAMPLES, LIME HILLS QUAdRANGLE, ALASKa--CONTINUEd Sample Au ppm-s B ppm-s Ba ppm-s Be ppm-s Bi ppm-s $\quad$ Cd ppm-s Co ppm-s Cr ppm-s Cu ppm-s

\begin{tabular}{|c|c|c|c|}
\hline LH0052C & N & N & 700 \\
\hline LH0053C & N & N & 700 \\
\hline LH0054C & N & N & 5,000 \\
\hline LH0055C & $\mathbf{N}$ & N & 700 \\
\hline LHO056C & 20 & N & 3,000 \\
\hline LH0057C & $\mathbf{N}$ & N & 700 \\
\hline LH0058C & N & $N$ & 300 \\
\hline LHOO59C & N & $\mathbf{N}$ & 300 \\
\hline LHOO6OC & N & N & 300 \\
\hline LHOOG1C & N & N & 200 \\
\hline LH0062C & N & 300 & 2,000 \\
\hline LH0063C & $N$ & 30 & 700 \\
\hline LH0064C & N & 30 & 300 \\
\hline LH0065C & N & N & 300 \\
\hline LH0066C & $N$ & $\mathbf{N}$ & 300 \\
\hline LH0067C & N & $\mathbf{N}$ & 100 \\
\hline LH0069C & N & N & 1,000 \\
\hline LHOOTOC & N & $\mathbf{N}$ & 700 \\
\hline LHO071C & N & 30 & 300 \\
\hline LHOO72C & N & $\mathbf{N}$ & 300 \\
\hline LHOOT3C & N & $\mathbf{N}$ & 200 \\
\hline LH0074C & N & $N$ & 300 \\
\hline LH0075C & N & N & 300 \\
\hline LH0076C & N & 20 & 300 \\
\hline LH0077C & N & 20 & 150 \\
\hline LH0O78C & N & $\mathbf{N}$ & 300 \\
\hline LH0079C & N & $N$ & 700 \\
\hline LHOOBOC & N & $N$ & 300 \\
\hline LHOOBIC & N & $N$ & 150 \\
\hline LH0082C & N & $\mathbf{N}$ & 300 \\
\hline LH0083C & $N$ & $N$ & 1,000 \\
\hline LH0084C & $\mathbf{N}$ & $N$ & $>10,000$ \\
\hline LH0085C & $N$ & $\mathbf{N}$ & 3,000 \\
\hline LH0086C & $\mathbf{N}$ & 20 & $>10,000$ \\
\hline LH0087C & $\mathbf{N}$ & 3,000 & $>10,000$ \\
\hline LH0088C & $\mathbf{N}$ & 100 & 3,000 \\
\hline LHOOB9C & $N$ & N & 500 \\
\hline LHOO9OC & $N$ & $N$ & 70 \\
\hline LH0091C & N & N & 70 \\
\hline LH0092C & N & 100 & $>10,000$ \\
\hline LH0093C & $\mathbf{N}$ & $N$ & $>10,000$ \\
\hline LH0094C & $\mathbf{N}$ & N & $>10,000$ \\
\hline LH0095C & $\mathbf{N}$ & 150 & $>10,000$ \\
\hline LH0096C & $\mathbf{N}$ & 150 & $>10,000$ \\
\hline LH0O97C & $\mathbf{N}$ & N & 7,000 \\
\hline LHO098C & N & 50 & $>10,000$ \\
\hline LH0099C & $\mathbf{N}$ & 50 & 1,000 \\
\hline LHO10OC & N & $N$ & 300 \\
\hline LH0101C & $\mathbf{N}$ & N & 7,000 \\
\hline LH0102C & $\mathbf{N}$ & 1,500 & $>10,000$ \\
\hline
\end{tabular}

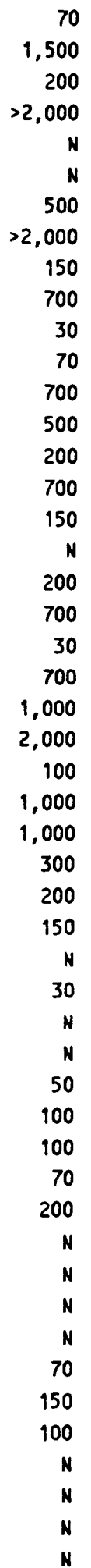

$<20$
$N$
$<20$
$<20$
$<20$
$<20$

70

$<20$

20

$<20$

30

$<20$

$<20$

$<20$

$<20$

$<20$

$<20$

$<20$

$<20$

$<20$

$<20$

$<20$

20

$<20$

$<20$

$<20$

$<20$

$<20$

$<20$

$<20$

$<20$

$<20$

$<20$

$<20$

$<20$

$<20$

$<20$

$<20$

$<20$

20

$<20$

$<20$

$<20$

$<20$

50

$<20$

$<20$

$<20$

$<20$

$<20$

30

100

$<20$

30

30
150

30

20

300

20

3,000

300

50

15

200

20

10

$<10$

$<10$

20

$<10$

50

$<10$

$<10$

$<10$

$<10$

15

$<10$

$<10$

20

30

10

$<10$

10

10

20

$<10$

10

30

30

150

$<10$

10

300

15

20

15

20

150

30

30

$<10$

$<10$

20 
TABLE 2. GEOCHEMICAL DATA FOR NONMAGNETIC, HEAVY-MINERAL-CONCENTRATE SAMPLES, LIME HILLS QUADRANGLE, ALASKA--CONTINUEd

Sample Ga ppm-s Ge ppm-s La ppm-s Mn ppm-s Mo ppm-s Nb ppm-s Ni ppm-s Pb ppm-s Pt ppm-s Sb ppm-s

\begin{tabular}{|c|c|c|c|c|c|c|c|c|}
\hline LH0052C & 20 & N & 1,500 & 5,000 & 700 & $<50$ & $<10$ & 2,000 \\
\hline LH0053C & $<10$ & 100 & $<100$ & 200 & 150 & $<50$ & $<10$ & 500 \\
\hline LH0054C & 10 & N & N & 150 & 150 & $<50$ & $<10$ & 700 \\
\hline LH0055C & 30 & $\mathbf{N}$ & 1,000 & 2,000 & 300 & 50 & $<10$ & 1,500 \\
\hline LH0056C & $<10$ & $\mathbf{N}$ & 200 & 200 & 30 & $<50$ & $<10$ & 1,500 \\
\hline LH0057C & 50 & $\mathbf{N}$ & 150 & 700 & 15 & 100 & $<10$ & 150 \\
\hline LH0058C & 20 & N & 1,500 & 1,000 & 200 & $<50$ & $<10$ & 5,000 \\
\hline LH0059C & 15 & N & 1,500 & 3,000 & 200 & 70 & $<10$ & 1,500 \\
\hline LH0060C & 10 & $\mathbf{N}$ & 1,500 & 700 & 700 & $<50$ & $<10$ & 3,000 \\
\hline LH0061C & $<10$ & N & N & 150 & 100 & $<50$ & $<10$ & 500 \\
\hline LH0062C & 30 & $N$ & 100 & 1,000 & 150 & $<50$ & 10 & 500 \\
\hline LH0063C & 20 & $N$ & 300 & 500 & N & $<50$ & 10 & 2,000 \\
\hline LH0064C & 10 & $\mathbf{N}$ & 150 & 150 & 50 & $<50$ & $<10$ & 3,000 \\
\hline LH0065C & 10 & $\mathbf{N}$ & 100 & 150 & 100 & $<50$ & $<10$ & 1,000 \\
\hline LH0066C & 10 & N & N & 100 & 200 & $<50$ & $<10$ & 300 \\
\hline LH0067C & $<10$ & N & 150 & 150 & 70 & $<50$ & $<10$ & 1,500 \\
\hline LH0069C & 15 & $\mathbf{N}$ & 100 & 150 & 150 & 50 & $<10$ & 70 \\
\hline LHOO70C & 15 & $\mathbf{N}$ & 1,500 & 700 & 700 & 50 & $<10$ & 100 \\
\hline LH0071C & 10 & $\mathbf{N}$ & 150 & 300 & 300 & 50 & $<10$ & 200 \\
\hline LH0072C & 10 & N & N & 150 & 500 & $<50$ & $<10$ & 500 \\
\hline LHOOT3C & $<10$ & $\mathbf{N}$ & 150 & 150 & 50 & $<50$ & $<10$ & N \\
\hline LH0074C & $<10$ & N & N & 100 & 150 & $<50$ & N & 200 \\
\hline LH0075C & $<10$ & $N$ & N & 150 & 150 & $<50$ & N & 700 \\
\hline LH0076C & 15 & N & 150 & 200 & 150 & $<50$ & $<10$ & 1,000 \\
\hline LH0077C & $<10$ & N & 150 & 150 & 30 & $<50$ & N & 70 \\
\hline LH0078C & $<10$ & N & N & 150 & 500 & $<50$ & $<10$ & 1,500 \\
\hline LH0079C & $<10$ & N & N & 150 & 50 & N & $<10$ & 1,500 \\
\hline LH0080C & 15 & N & 100 & 1,500 & N & $<50$ & $<10$ & $<20$ \\
\hline LH0081C & 10 & N & 700 & 700 & 700 & 50 & $<10$ & 200 \\
\hline LH0082C & $<10$ & N & 150 & 150 & 30 & $<50$ & N & 200 \\
\hline LH0083C & 15 & N & 150 & 150 & 200 & 50 & N & 20 \\
\hline LH0084C & $<10$ & N & N & 70 & 50 & $<50$ & N & 1,500 \\
\hline LHO085C & $<10$ & N & 100 & 100 & 20 & $<50$ & N & 500 \\
\hline LH0086C & $<10$ & N & 200 & 70 & 15 & $<50$ & $<10$ & 500 \\
\hline LH0087C & 20 & N & N & 150 & 50 & $<50$ & 10 & 7,000 \\
\hline LH0088C & $<10$ & N & 700 & 100 & 50 & $<50$ & 30 & 5,000 \\
\hline LH0089C & $<10$ & $\mathbf{N}$ & N & 50 & 50 & $<50$ & 500 & 200 \\
\hline LHO090C & $N$ & $\mathbf{N}$ & 300 & 70 & 100 & $<50$ & N & 300 \\
\hline LH0091C & $N$ & N & 300 & 70 & 100 & $<50$ & N & 2,000 \\
\hline LH0092C & $<10$ & $\mathbf{N}$ & 150 & 150 & 20 & $<50$ & 200 & 200 \\
\hline LH0093C & N & $\mathbf{N}$ & N & 150 & N & $<50$ & 10 & 50 \\
\hline LH0094C & N & N & N & 100 & N & $<50$ & 10 & 150 \\
\hline LH0095C & $<10$ & $\mathbf{N}$ & 150 & 200 & 15 & $<50$ & 10 & 1,500 \\
\hline LH0096C & $<10$ & $N$ & N & 200 & 20 & $<50$ & 20 & 15,000 \\
\hline LH0097C & $<10$ & $\mathbf{N}$ & N & 100 & 300 & $<50$ & 200 & 15,000 \\
\hline LH0098C & 15 & $\mathbf{N}$ & N & 200 & 100 & 50 & $<10$ & 9,500 \\
\hline LH0099C & 20 & $\mathbf{N}$ & N & 500 & N & $<50$ & 10 & 150 \\
\hline LH0100C & N & $\mathbf{N}$ & 150 & 70 & 100 & $<50$ & N & 200 \\
\hline LH0101C & $\mathbf{N}$ & N & 150 & 150 & N & $<50$ & $\mathbf{N}$ & N \\
\hline LH0102C & 20 & $\mathbf{N}$ & 100 & 150 & 100 & 100 & 10 & 200 \\
\hline
\end{tabular}


tABLE 2. GEOCHEMICAL DATA FOR NONMAGNETIC, hEAVY-MINERAL-CONCENTRATE SAMPLES, Lime hILlS QUAdRANGLE, ALASKA--ContinUed Sc ppm-s

Sn ppm-s

Sr ppm-s

Th ppm-s

u ppm-s V ppm-s

ppm-s

Y ppm-s

Zn ppm-s $\quad$ zr ppm-s

\begin{tabular}{|c|c|c|c|c|c|c|c|c|c|c|}
\hline LH0052C & 70 & 700 & $<200$ & 500 & $N$ & 70 & 70 & 1,500 & $N$ & $>2,000$ \\
\hline LH0053C & $<10$ & 700 & $<200$ & N & N & $<20$ & 70 & 300 & $N$ & $>2,000$ \\
\hline LHO054C & 70 & 700 & $<200$ & 1,500 & 2,000 & $N$ & 150 & 1,000 & $N$ & $>2,000$ \\
\hline LH0055C & 50 & 30 & 300 & 1,000 & 1,000 & 50 & 300 & 500 & N & $>2,000$ \\
\hline LH0056C & 100 & 100 & $<200$ & N & N & 30 & $N$ & 700 & 700 & $>2,000$ \\
\hline LH0057C & 70 & 200 & $<200$ & 200 & N & 70 & $N$ & 300 & N & $>2,000$ \\
\hline LH0058C & 150 & 700 & $<200$ & 1,500 & 2,000 & $<20$ & N & $>5,000$ & 10,000 & $>2,000$ \\
\hline LH0059C & 150 & $>2,000$ & $<200$ & 3,000 & 3,000 & 20 & 1,500 & 5,000 & 2,000 & $>2,000$ \\
\hline LH0060C & 150 & 500 & $<200$ & $>5,000$ & 5,000 & 20 & 70 & 3,000 & 1.000 & $>2,000$ \\
\hline LH0061C & 70 & 700 & $<200$ & 700 & 1,500 & N & 150 & 3,000 & 1,500 & $>2,000$ \\
\hline LH0062C & 20 & 20 & 200 & $N$ & $N$ & 100 & 70 & 300 & $N$ & $>2,000$ \\
\hline LH0063C & 15 & 20 & 1,500 & N & N & 70 & 700 & 300 & $N$ & $>2,000$ \\
\hline LH0064C & 70 & 1,500 & $<200$ & 700 & 2,000 & $<20$ & 300 & 1,000 & $N$ & $>2,000$ \\
\hline LH0065C & 70 & 1,000 & $<200$ & 700 & 2,000 & N & 200 & 1,000 & $N$ & $>2,000$ \\
\hline LH0066C & 30 & 500 & $<200$ & N & N & $N$ & 500 & 500 & N & $>2,000$ \\
\hline LH0067C & 70 & $>2,000$ & $<200$ & 500 & N & $N$ & 150 & 1.500 & 700 & $>2,000$ \\
\hline LH0069C & 30 & 500 & 200 & 1,500 & 1,500 & $<20$ & 15,000 & 700 & N & $>2,000$ \\
\hline LHOOTOC & 70 & 200 & 200 & 700 & 1,000 & $<20$ & 1,000 & 700 & $N$ & $>2,000$ \\
\hline LH0071C & 50 & 300 & 200 & 200 & N & $<20$ & 1,000 & 700 & $N$ & $>2,000$ \\
\hline LH0072C & 30 & $N$ & $<200$ & 200 & $N$ & $<20$ & 500 & 700 & N & $>2,000$ \\
\hline LH0OT3C & 30 & 20 & $<200$ & 200 & N & 20 & 150 & 700 & N & $>2,000$ \\
\hline LH0074C & 30 & 1,000 & $<200$ & 200 & $N$ & $<20$ & 1,000 & 700 & 1,500 & $>2,000$ \\
\hline LH0075C & 70 & 1,500 & $N$ & 200 & N & N & 300 & 700 & 10,000 & $>2,000$ \\
\hline LHOOT6C & 70 & 30 & $<200$ & N & N & $<20$ & 2,000 & 700 & N & $>2,000$ \\
\hline LH0O77C & 150 & 20 & $<200$ & 500 & 1,000 & $<20$ & 500 & 1,500 & $N$ & $>2,000$ \\
\hline LH0O78C & 70 & 150 & $<200$ & N & $<1,000$ & $<20$ & 1,000 & 1,000 & N & $>2,000$ \\
\hline LH0079C & 30 & 1,000 & $<200$ & 300 & N & $<20$ & 500 & 500 & 7,000 & $>2,000$ \\
\hline LH0080C & $<10$ & 50 & 300 & N & N & 30 & 150 & 700 & N & $>2,000$ \\
\hline LH0081C & 30 & 300 & 300 & $<200$ & N & 20 & 1,000 & 700 & $N$ & $>2,000$ \\
\hline LH0082C & 70 & 300 & $<200$ & 200 & 1,000 & $N$ & 300 & 700 & N & $>2,000$ \\
\hline LH0083C & 20 & N & 300 & 700 & N & $<20$ & 3,000 & 300 & N & $>2,000$ \\
\hline LH0084C & 30 & $N$ & 200 & N & $N$ & N & 300 & 500 & N & $>2,000$ \\
\hline LH0085C & 15 & N & $<200$ & N & N & N & 700 & 1,000 & 2,000 & $>2,000$ \\
\hline LH0086C & $N$ & $N$ & 1,000 & 700 & N & $<20$ & N & 1,500 & 700 & $>2,000$ \\
\hline LH0087C & 10 & N & 7,000 & N & N & 70 & 500 & 150 & $N$ & $>2,000$ \\
\hline LH0088C & 10 & 200 & 200 & 500 & $<1,000$ & $<20$ & 100 & 2,000 & 1.000 & $>2,000$ \\
\hline LH0089C & N & $N$ & N & N & N & 20 & 70 & 100 & N & $>2,000$ \\
\hline LH0090C & $N$ & N & $<200$ & 700 & N & N & 200 & 1,500 & $N$ & $>2,000$ \\
\hline LH0091C & 10 & 200 & $<200$ & $>5,000$ & 3,000 & $N$ & $N$ & 1,500 & N & $>2,000$ \\
\hline LH0092C & $<10$ & N & 1.500 & N & N & 70 & N & 200 & 1,000 & $>2,000$ \\
\hline LH0093C & $N$ & N & 7,000 & $N$ & $N$ & 20 & N & 50 & $N$ & 1,500 \\
\hline LH0094C & $<10$ & N & 7,000 & $N$ & $N$ & 50 & $N$ & 100 & 1,000 & 500 \\
\hline LH0095C & 15 & N & 2,000 & $<200$ & $N$ & 70 & N & 700 & N & $>2,000$ \\
\hline LH0096C & 10 & 700 & 5,000 & N & $N$ & 150 & N & 200 & $N$ & $>2,000$ \\
\hline LH0097C & $N$ & N & N & $N$ & $N$ & $<20$ & 1,000 & 300 & $N$ & $>2,000$ \\
\hline LH0098C & 15 & 300 & 1,500 & $N$ & $N$ & 150 & 1,000 & 100 & $N$ & $>2,000$ \\
\hline LH0099C & 20 & N & 2,000 & $N$ & N & 200 & 150 & 70 & $N$ & $>2,000$ \\
\hline LHO100C & 70 & $N$ & $N$ & 500 & 1,000 & $<20$ & 500 & 1.000 & $N$ & $>2,000$ \\
\hline LH0101C & 50 & 70 & $<200$ & 500 & 1,000 & 20 & 150 & 1,000 & N & $>2,000$ \\
\hline LH0102C & $<10$ & $N$ & 700 & N & N & 50 & $>20,000$ & 100 & N & $>2,000$ \\
\hline
\end{tabular}


TABLE 2. GEOCHEMICAL DATA FOR NONMAGNETIC, hEAVY-MINERAL-CONCENTRATE SAMPLES, LIME hILLS QUADRANGLE, ALASKA--ContinUEd

Sample Latitude Longitude $\mathrm{Fe} \%$-s $\mathrm{Mg}$ \%-s $\mathrm{Ca}$ \%-s $\mathrm{Na}$ \%-s $\quad$ Ti $\%$-s $\quad$ P $\quad$-s Ag ppm-s As ppm-s

\begin{tabular}{|c|c|c|c|c|c|c|c|c|c|c|c|c|}
\hline LH0103C & 6142 & 55 & 153 & 4150 & 1.00 & .20 & 15.00 & 1.5 & .700 & 5.0 & 10.0 & N \\
\hline LH0104C & 6142 & 57 & 153 & 4040 & .70 & .05 & 10.00 & .7 & .700 & 3.0 & 300.0 & 2,000 \\
\hline LH0105C & 6142 & 24 & 153 & 3741 & .70 & .10 & 15.00 & .7 & 1.000 & 5.0 & 100.0 & 1,000 \\
\hline LHO106C & 6138 & 9 & 153 & 3441 & .30 & .05 & 7.00 & $<.5$ & 1.500 & 5.0 & 500.0 & N \\
\hline LH0107C & 6138 & 17 & 153 & 3447 & 7.00 & .15 & .30 & $<.5$ & .200 & $N$ & 150.0 & 1,000 \\
\hline LHO108C & 6135 & 57 & 153 & 3640 & 1.00 & .10 & 1.00 & $<.5$ & .300 & 1.0 & 5.0 & 500 \\
\hline LHO109C & 6136 & 48 & 153 & 3027 & .50 & .07 & 1.50 & $<.5$ & $>2.000$ & 3.0 & 200.0 & N \\
\hline LHO1 10C & 6132 & 27 & 153 & 3757 & 3.00 & .20 & 10.00 & .7 & 2.000 & 5.0 & 100.0 & 1,500 \\
\hline LHO115C & 6136 & 22 & 153 & 239 & 7.00 & .15 & 20.00 & .7 & .500 & 20.0 & 100.0 & $>20,000$ \\
\hline LH0116C & 6133 & 16 & 153 & 1838 & 7.00 & .07 & 20.00 & 1.5 & .100 & 7.0 & 300.0 & 7,000 \\
\hline LHO117C & 6141 & 44 & 153 & 2015 & 1.00 & .05 & 15.00 & .7 & 1.500 & 5.0 & 15.0 & 700 \\
\hline LHO118C & 6139 & 23 & 153 & 1555 & 7.00 & .15 & 10.00 & .7 & $>2.000$ & 5.0 & 200.0 & 1,500 \\
\hline LHO119C & 6142 & 19 & 153 & 1646 & 7.00 & 1.50 & 15.00 & .7 & 1.000 & 1.5 & N & 2,000 \\
\hline LH0120C & 6130 & 29 & 153 & 811 & 7.00 & .10 & 15.00 & 1.0 & 2.000 & 1.5 & 50.0 & 2,000 \\
\hline LH0121C & 6130 & 39 & 153 & 99 & 3.00 & .15 & 7.00 & 1.5 & 1.500 & 2.0 & 2.0 & N \\
\hline LHO122C & 6132 & 11 & 153 & 722 & .50 & .05 & 20.00 & 1.0 & .300 & 20.0 & 15.0 & $<500$ \\
\hline LHO123C & 6132 & 22 & 153 & 034 & 5.00 & $<.05$ & 30.00 & .7 & 1.000 & 1.5 & 150.0 & 7,000 \\
\hline LH0124C & 6132 & 49 & 153 & 046 & .50 & $<.05$ & 50.00 & $<.5$ & 2.000 & 1.0 & 150.0 & 1,000 \\
\hline LH0125C & 6132 & 59 & 153 & 043 & .30 & .05 & 30.00 & .7 & 2.000 & 7.0 & N & $N$ \\
\hline LHO126C & 6136 & 26 & 153 & 537 & 1.50 & .10 & 15.00 & .7 & 1.500 & 7.0 & 20.0 & 3,000 \\
\hline LHO127C & 6159 & 59 & 153 & 5038 & 1.00 & 1.50 & 10.00 & $<.5$ & .200 & 3.0 & $N$ & N \\
\hline LHO128C & 6158 & 1 & 153 & 4712 & 3.00 & .20 & 20.00 & .7 & 2.000 & 10.0 & 10.0 & 7,000 \\
\hline LH0129C & 6158 & 0 & 153 & 4742 & 7.00 & .05 & 2.00 & .5 & .300 & 1.0 & 15.0 & 20,000 \\
\hline LHO130C & 6159 & 43 & 153 & 4333 & 7.00 & .30 & 7.00 & .5 & .500 & .5 & $N$ & 500 \\
\hline LH0131C & 6159 & 42 & 153 & 4049 & 2.00 & .15 & 5.00 & $<.5$ & .100 & .7 & N & N \\
\hline LH0132C & 6157 & 57 & 153 & 3341 & 1.00 & .07 & 20.00 & .7 & 2.000 & 20.0 & 50.0 & 700 \\
\hline LHO133C & 6156 & 29 & 153 & 3245 & 7.00 & .20 & 30.00 & $<.5$ & 1.500 & 7.0 & 50.0 & 7,000 \\
\hline LH0134C & 6155 & 42 & 153 & 3857 & .20 & $<.05$ & 5.00 & $\mathbf{N}$ & .100 & .7 & 10.0 & 700 \\
\hline LH0135C & 6151 & 41 & 153 & 4512 & 15.00 & .15 & 3.00 & $<.5$ & .700 & $N$ & 700.0 & $>20,000$ \\
\hline LHO136C & 6151 & 57 & 153 & 4620 & .50 & $<.05$ & 2.00 & $N$ & .300 & 3.0 & 15.0 & 7,000 \\
\hline LH0137C & 6152 & 2 & 153 & 4040 & 20.00 & .20 & 3.00 & $<.5$ & $>2.000$ & 2.0 & 15.0 & $>20,000$ \\
\hline LHO138C & 6153 & 7 & 153 & 3252 & 7.00 & 5.00 & 7.00 & 1.0 & .700 & 1.0 & N & 2,000 \\
\hline LH0139C & 6148 & 56 & 153 & 410 & 2.00 & 1.00 & 1.00 & $<.5$ & .200 & $\mathbf{N}$ & N & $<500$ \\
\hline LHO140C & 6122 & 5 & 153 & 1837 & 5.00 & .30 & 2.00 & .7 & 2.000 & 1.0 & 20.0 & 1,000 \\
\hline LH0141C & 6123 & 9 & 153 & 1657 & .30 & $<.05$ & .50 & 1.0 & .100 & .5 & 3.0 & 700 \\
\hline LHO142C & 6123 & 12 & 153 & 63 & .30 & $<.05$ & .50 & .5 & .070 & .7 & $N$ & $<500$ \\
\hline LH0143C & 6119 & 28 & 153 & 922 & 1.00 & $<.05$ & 1.00 & $N$ & .150 & 1.5 & 7.0 & 3,000 \\
\hline LHO144C & 6120 & 21 & 153 & 1130 & .50 & $<.05$ & .70 & $N$ & .100 & 1.5 & 5.0 & 3,000 \\
\hline LHO145C & 6118 & 23 & 153 & 1313 & 10.00 & .20 & 7.00 & .7 & 2.000 & 3.0 & 700.0 & $>20,000$ \\
\hline LHO146C & 6119 & 22 & 153 & 120 & .50 & .10 & 10.00 & 1.5 & .300 & 3.0 & 20.0 & N \\
\hline LH0147C & 6133 & 18 & 153 & 1248 & 10.00 & .70 & 20.00 & .7 & 1.500 & 7.0 & 15.0 & 500 \\
\hline LH0152C & 6124 & 35 & 153 & 4253 & 1.00 & .15 & 15.00 & .5 & $>2.000$ & 1.0 & $N$ & 500 \\
\hline LH0153C & 6122 & 21 & 153 & 4318 & 7.00 & .70 & 20.00 & .7 & 2.000 & 7.0 & $N$ & 700 \\
\hline LH0154C & 6121 & 49 & 153 & 3730 & 5.00 & .20 & 10.00 & 1.0 & $>2.000$ & 2.0 & 5.0 & 1,000 \\
\hline LH0155C & 6123 & 7 & 153 & 3355 & 30.00 & $<.05$ & 1.00 & $<.5$ & 2.000 & $N$ & 30.0 & 1,500 \\
\hline LH0156C & 6124 & 16 & 153 & 3312 & 15.00 & .07 & 2.00 & $<.5$ & 1.500 & 1.5 & 30.0 & $>20,000$ \\
\hline LH0157C & 6124 & 24 & 153 & 3053 & 7.00 & $<.05$ & 1.00 & $<.5$ & .700 & .7 & 2.0 & 1,000 \\
\hline LH0158C & 6125 & 26 & 153 & 2922 & 7.00 & .20 & 10.00 & .7 & $>2.000$ & 1.0 & 10.0 & $>20,000$ \\
\hline LH0159C & 6125 & 48 & 153 & 3544 & 5.00 & .30 & 20.00 & 1.0 & $>2.000$ & 1.5 & 5.0 & 1,000 \\
\hline LH0160C & 6127 & 26 & 153 & 3444 & 7.00 & .20 & 15.00 & .7 & $>2.000$ & 3.0 & 70.0 & 1,500 \\
\hline
\end{tabular}




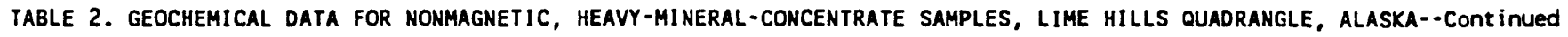

\begin{tabular}{|c|c|c|c|c|c|c|c|c|c|}
\hline Sample & Au ppm-s & ppm-s & Ba ppm-s & Be ppm-s & Bi ppm-s & Cd ppm-s & Co ppm-s & $\mathrm{Cr}$ ppm-s & Cu ppm-s \\
\hline LH0103C & N & 700 & 5,000 & 2 & $N$ & N & $<20$ & 100 & 15 \\
\hline LH0104C & 500 & 500 & $>10,000$ & $\mathbf{N}$ & $<20$ & $N$ & 20 & 70 & 15 \\
\hline LH0105C & 150 & 200 & $>10,000$ & $\mathbf{N}$ & 100 & $N$ & $\mathbf{N}$ & 50 & 15 \\
\hline LHO106C & N & 20 & $>10,000$ & $N$ & 1,500 & $N$ & $\mathbf{N}$ & 30 & 30 \\
\hline LH0107C & N & 30 & $>10,000$ & N & 30 & $N$ & 30 & 150 & 30 \\
\hline LHO108C & N & 30 & 1,000 & 3 & 500 & $N$ & 30 & 200 & 15 \\
\hline LH0109C & N & 20 & $>10,000$ & N & N & $N$ & $<20$ & 30 & 10 \\
\hline LHO110C & N & 50 & $>10,000$ & 3 & 200 & $N$ & 50 & 70 & 2,000 \\
\hline LHO115C & 20 & 30 & $>10,000$ & $N$ & 150 & $N$ & 150 & 30 & 150 \\
\hline LHO116C & $N$ & N & $>10,000$ & $N$ & 700 & $N$ & 300 & $<20$ & 100 \\
\hline LHO117C & $N$ & $N$ & 700 & 2 & 700 & $\mathbf{N}$ & $N$ & $<20$ & 15 \\
\hline LHO118C & 150 & $\mathbf{N}$ & $>10,000$ & N & 200 & N & 30 & 20 & 70 \\
\hline LHO119C & $N$ & 50 & 500 & 5 & 30 & $N$ & $<20$ & 200 & 50 \\
\hline LHO120C & $N$ & $\mathbf{N}$ & 5,000 & 3 & 30 & $N$ & 300 & $<20$ & 100 \\
\hline LH0121C & $N$ & N & 700 & 7 & $<50$ & $N$ & $<50$ & 70 & 70 \\
\hline LH0122C & $N$ & N & 1,500 & $N$ & 100 & $N$ & $<20$ & $<20$ & 10 \\
\hline LH0123C & $N$ & N & 150 & $N$ & 1,000 & $N$ & $<20$ & $<20$ & 70 \\
\hline LHO124C & N & N & 50 & $N$ & 200 & $N$ & N & $<20$ & 10 \\
\hline LH0125C & N & N & $<50$ & 2 & N & N & $N$ & $<20$ & $<10$ \\
\hline LH0126C & N & 50 & 200 & 3 & 500 & N & 30 & $<20$ & 100 \\
\hline LH0127C & $N$ & 200 & $>10,000$ & N & $N$ & N & $N$ & $<20$ & 20 \\
\hline LHO128C & N & 500 & $>10,000$ & N & 150 & $N$ & 200 & 70 & 150 \\
\hline LH0129C & N & 50 & 7,000 & 2 & 500 & $\mathbf{N}$ & 700 & $<20$ & 70 \\
\hline LHO130C & N & 50 & $>10,000$ & $N$ & N & N & 30 & 50 & 50 \\
\hline LHO131C & N & $<20$ & $>10,000$ & $N$ & N & $N$ & $<20$ & $<20$ & 15 \\
\hline LH0132C & N & 20 & $>10,000$ & N & 700 & $N$ & 50 & 50 & 10 \\
\hline H0133C & N & 20 & $>10,000$ & N & 200 & N & 300 & 20 & 100 \\
\hline H0134C & N & $N$ & 300 & 3 & 500 & N & N & N & 10 \\
\hline H0135C & $N$ & 300 & 500 & $N$ & $>2,000$ & 100 & 1,500 & $<20$ & 3,000 \\
\hline L $0136 \mathrm{C}$ & N & $N$ & 500 & $N$ & 300 & $N$ & 70 & 20 & 30 \\
\hline H0137C & $N$ & 300 & 150 & $N$ & 150 & $N$ & 2,000 & 100 & 500 \\
\hline H0138C & $N$ & 5,000 & 500 & 3 & $N$ & $N$ & 50 & 1,500 & 100 \\
\hline H0139C & N & 3,000 & 100 & $N$ & $N$ & $N$ & $<20$ & 30 & 30 \\
\hline HO140C & N & N & 300 & 10 & 2,000 & 100 & 30 & $<20$ & 300 \\
\hline H0141C & N & N & 300 & 3 & 700 & N & N & $N$ & 20 \\
\hline H0142C & $N$ & $N$ & 150 & 3 & 200 & N & $N$ & N & 10 \\
\hline H0143C & N & N & 150 & 3 & 200 & N & N & N & 20 \\
\hline H0144C & $\mathbf{N}$ & N & 70 & 3 & 300 & N & $N$ & $N$ & 30 \\
\hline $\mathrm{H} 0145 \mathrm{C}$ & N & 100 & 150 & 2 & 500 & N & 70 & $<20$ & 2,000 \\
\hline.$H 0146 C$ & $N$ & $N$ & 300 & $N$ & 150 & $N$ & $N$ & $<20$ & 10 \\
\hline $\mathrm{H} 0147 \mathrm{C}$ & N & $N$ & $>10,000$ & 5 & 100 & N & 50 & 70 & 300 \\
\hline H0152C & $N$ & $N$ & 150 & $N$ & $N$ & $N$ & 30 & 100 & 70 \\
\hline.$H 0153 C$ & N & 100 & 300 & 3 & 70 & $N$ & 150 & 20 & 50 \\
\hline HO154C & $N$ & 70 & 500 & 10 & $N$ & 50 & 30 & 70 & 150 \\
\hline $\mathrm{H} 0155 \mathrm{C}$ & N & N & 1,500 & 3 & $\mathbf{N}$ & 50 & 50 & $<20$ & 150 \\
\hline $.40156 \mathrm{C}$ & N & 300 & 150 & $N$ & 500 & 70 & 700 & 30 & 200 \\
\hline $.40157 \mathrm{C}$ & $N$ & N & 700 & N & $N$ & 150 & $<20$ & $N$ & 30 \\
\hline $.40158 \mathrm{C}$ & N & 100 & 1,000 & 7 & N & N & 30 & 150 & 200 \\
\hline H0159C & $N$ & 300 & 300 & 7 & $N$ & $N$ & $<20$ & 70 & 300 \\
\hline. $\mathrm{H} 0160 \mathrm{C}$ & $N$ & 1,000 & 2,000 & 10 & 500 & $\mathbf{N}$ & 50 & 70 & 150 \\
\hline
\end{tabular}


TABLE 2. GEOCHEMICAL DATA FOR NONMAGNETIC, hEAVY-MINERAL-CONCENTRATE SAMPLES, LIME hILLS QUADRANGLE, ALASKA--CONTInUEd

Sample Ga ppm-s Ge ppm-s La ppm-s Mn ppm-s Mo ppm-s Nb ppm-s Ni ppm-s Pb ppm-s Pt ppm-s Sb ppm-s

\begin{tabular}{|c|c|c|c|c|c|c|c|c|c|c|}
\hline LH0103C & 30 & $\mathbf{N}$ & 150 & 300 & 300 & 200 & 10 & 300 & $N$ & N \\
\hline LHO104C & $<10$ & $\mathbf{N}$ & 100 & 150 & 15 & 100 & 10 & 300 & N & N \\
\hline LH0105C & $<10$ & $\mathbf{N}$ & 150 & 150 & 150 & 150 & $<10$ & 3,000 & N & N \\
\hline LH0106C & $<10$ & N & $<100$ & 150 & $N$ & $<50$ & $<10$ & $>50,000$ & $N$ & 200 \\
\hline LH0107C & 50 & N & $N$ & 100 & $\mathbf{N}$ & $<50$ & 20 & 2,000 & $N$ & $<200$ \\
\hline LHO108C & 50 & $N$ & $N$ & 150 & 15 & $<50$ & 10 & 300 & N & N \\
\hline LH0109C & $N$ & $N$ & $N$ & 150 & N & 200 & 10 & 700 & $N$ & N \\
\hline LH0110C & 20 & $\mathbf{N}$ & 300 & 700 & 300 & $<50$ & 20 & 30,000 & $\mathbf{N}$ & N \\
\hline LH0115C & $\mathbf{N}$ & $\mathbf{N}$ & 1,000 & 500 & $\mathbf{N}$ & $<50$ & 30 & 7,000 & $\mathbf{N}$ & $<200$ \\
\hline LH0116C & $\mathbf{N}$ & $\mathbf{N}$ & 500 & 700 & $N$ & $<50$ & 30 & 30,000 & $N$ & $<200$ \\
\hline LH0117C & $<10$ & $\mathbf{N}$ & 300 & 300 & N & 50 & N & 1,000 & $\mathbf{N}$ & N \\
\hline LH0118C & $<10$ & $\mathbf{N}$ & 300 & 500 & $\mathbf{N}$ & 50 & $<10$ & 3,000 & $\mathbf{N}$ & $N$ \\
\hline LHO119C & 50 & $\mathbf{N}$ & 200 & 2,000 & 50 & 50 & $<10$ & 700 & $N$ & N \\
\hline LHO120C & 20 & $\mathbf{N}$ & 300 & 700 & 20 & 50 & $<10$ & 2,000 & $\mathbf{N}$ & N \\
\hline LH0121C & $<20$ & $\mathbf{N}$ & 200 & 1,000 & N & 70 & $<20$ & 1,500 & $\mathbf{N}$ & $N$ \\
\hline LH0122C & $<10$ & $\mathbf{N}$ & 700 & 1,000 & 150 & $<50$ & N & 2,000 & $\mathbf{N}$ & $N$ \\
\hline LH0123C & N & $\mathbf{N}$ & 300 & 200 & 100 & 100 & $\mathbf{N}$ & 3,000 & N & N \\
\hline LH0124C & $<10$ & $\mathbf{N}$ & 200 & 150 & 500 & 150 & $N$ & 1,000 & $\mathbf{N}$ & $N$ \\
\hline LH0125C & $<10$ & $\mathbf{N}$ & 500 & 700 & $\mathbf{N}$ & 100 & N & 50 & N & $\mathbf{N}$ \\
\hline LH0126C & $<10$ & $\mathbf{N}$ & 700 & 1,000 & 30 & 50 & $<10$ & 5,000 & N & N \\
\hline LH0127C & $<10$ & $\mathbf{N}$ & $<100$ & 150 & 15 & $<50$ & 10 & 100 & N & N \\
\hline LH0128C & $<10$ & $N$ & 150 & 1,000 & $\mathbf{N}$ & 50 & 300 & 700 & N & 700 \\
\hline LH0129C & N & $\mathbf{N}$ & 100 & 100 & 150 & 50 & 200 & 1,500 & N & N \\
\hline LH0130C & $<10$ & N & 150 & 150 & N & $<50$ & 30 & 700 & $\mathbf{N}$ & $\mathbf{N}$ \\
\hline LH0131C & $\mathbf{N}$ & N & N & 100 & $\mathbf{N}$ & $<50$ & 10 & $<20$ & $N$ & N \\
\hline LHO132C & N & $\mathbf{N}$ & 700 & 200 & $\mathbf{N}$ & $<50$ & 10 & 300 & $N$ & N \\
\hline LH0133C & $<10$ & $\mathbf{N}$ & 100 & 200 & N & $<50$ & 100 & 10,000 & $\mathbf{N}$ & $<200$ \\
\hline LH0134C & N & N & 150 & 70 & 700 & 50 & N & 1,000 & $\mathbf{N}$ & N \\
\hline LH0135C & $\mathbf{N}$ & $\mathbf{N}$ & 100 & 150 & 500 & 70 & 700 & 10,000 & $\mathbf{N}$ & $<200$ \\
\hline LH0136C & $\mathbf{N}$ & $\mathbf{N}$ & $\mathbf{N}$ & 100 & 100 & 70 & 20 & 500 & $\mathbf{N}$ & N \\
\hline LH0137C & $\mathbf{N}$ & $\mathbf{N}$ & $N$ & 150 & 20 & 70 & 1,500 & 1,000 & $\mathbf{N}$ & $<200$ \\
\hline LH0138C & 15 & $\mathbf{N}$ & 300 & 1,000 & $\mathbf{N}$ & $<50$ & 50 & 50 & $N$ & N \\
\hline LH0139C & $\mathbf{N}$ & $\mathbf{N}$ & 300 & 500 & N & $<50$ & $<10$ & N & N & N \\
\hline LHO140C & 20 & $\mathbf{N}$ & 500 & 3,000 & 700 & 50 & $<10$ & 7,000 & $\mathbf{N}$ & N \\
\hline LHO141C & $<10$ & $\mathbf{N}$ & $\mathbf{N}$ & 150 & 50 & $<50$ & $\mathbf{N}$ & 300 & $\mathbf{N}$ & N \\
\hline LHO142C & N & $\mathbf{N}$ & N & 150 & 70 & $<50$ & N & 300 & N & N \\
\hline LH0143C & N & $\mathbf{N}$ & 300 & 200 & 500 & $<50$ & $<10$ & 700 & $\mathbf{N}$ & N \\
\hline LHO144C & $\mathbf{N}$ & $\mathbf{N}$ & 700 & 300 & 200 & $<50$ & N & 150 & N & N \\
\hline LHO145C & $<10$ & $\mathbf{N}$ & 700 & 3,000 & 1,500 & $<50$ & $<10$ & 40,000 & $N$ & N \\
\hline LHO146C & 10 & $\mathbf{N}$ & 300 & 500 & 50 & $<50$ & $<10$ & 150 & $\mathbf{N}$ & N \\
\hline LH0147C & 30 & $\mathbf{N}$ & 1,500 & 2,000 & 70 & $<50$ & 30 & 3,000 & $N$ & N \\
\hline LHO152C & $<10$ & $N$ & 700 & 700 & 50 & 200 & $<10$ & 150 & $\mathbf{N}$ & N \\
\hline LHO153C & 20 & $\mathbf{N}$ & 300 & 700 & 50 & 50 & 10 & 50 & $\mathbf{N}$ & $\mathbf{N}$ \\
\hline LHO154C & 20 & $\mathbf{N}$ & 1,500 & 1,500 & 30 & 500 & 20 & 1,000 & N & $\mathbf{N}$ \\
\hline LHO155C & 10 & $\mathbf{N}$ & 150 & 150 & 50 & 50 & $<10$ & 1,500 & $\mathbf{N}$ & $\mathbf{N}$ \\
\hline LH0156C & $\mathbf{N}$ & $N$ & 200 & 100 & $\mathbf{N}$ & $<50$ & 700 & 3,000 & $\mathbf{N}$ & 300 \\
\hline LHO157C & $\mathbf{N}$ & $\mathbf{N}$ & 150 & 100 & $\mathbf{N}$ & $<50$ & $<10$ & 100 & $\mathbf{N}$ & $N$ \\
\hline LH0158C & 10 & $\mathbf{N}$ & 300 & 500 & 50 & 100 & 10 & 1,500 & $\mathbf{N}$ & $<200$ \\
\hline LH0159C & 50 & $\mathbf{N}$ & 300 & 1,000 & 70 & 100 & $<10$ & 1,000 & $\mathbf{N}$ & N \\
\hline LHO160C & 50 & $\mathbf{N}$ & 300 & 500 & 50 & 70 & 10 & 2,000 & $\mathbf{N}$ & $\mathbf{N}$ \\
\hline
\end{tabular}


TABLE 2. GEOCHEMICAL DATA FOR NONMAGNETIC, HEAVY-MINERAL-CONCENTRATE SAMPLES, LIME hILLS QUADRANGLE, ALASKA--CONTINUEd

Sample Sc ppm-s Sn ppm-s Sr ppm-s Th ppm-s $U$ ppom-s $V$ ppm-s W ppm-s Y ppm-s Zn ppm-s Zr ppm-s

\begin{tabular}{|c|c|c|c|c|c|c|c|c|c|c|}
\hline LH0103C & 10 & $N$ & 1,500 & $N$ & N & 150 & $>20,000$ & 200 & $N$ & $>2,000$ \\
\hline LH0104C & 15 & 300 & 1,500 & $\mathbf{N}$ & $\mathbf{N}$ & 50 & 20,000 & 300 & N & $>2,000$ \\
\hline LH0105C & $<10$ & N & 1,500 & $\mathbf{N}$ & $\mathbf{N}$ & 30 & $>20,000$ & 200 & $\mathbf{N}$ & $>2,000$ \\
\hline LHO106C & 15 & 1,000 & 1,500 & N & $\mathbf{N}$ & 50 & 200 & 200 & $N$ & $>2,000$ \\
\hline LH0107C & $\mathbf{N}$ & 700 & 200 & $\mathbf{N}$ & $\mathbf{N}$ & 150 & 200 & 30 & $\mathbf{N}$ & $>2,000$ \\
\hline LHO108C & $\mathbf{N}$ & N & $<200$ & $N$ & $\mathbf{N}$ & 150 & 700 & 100 & $\mathbf{N}$ & $>2,000$ \\
\hline LH0109C & 50 & $\mathbf{N}$ & 1,500 & $N$ & N & 70 & 70 & 200 & N & $>2,000$ \\
\hline LHO110C & 30 & N & 500 & $N$ & $N$ & 150 & 200 & 300 & 700 & $>2,000$ \\
\hline LH0115C & 15 & 700 & 700 & $N$ & $\mathbf{N}$ & 70 & 200 & 500 & N & $>2,000$ \\
\hline LHO116C & 20 & 500 & 1,000 & $\mathbf{N}$ & $\mathbf{N}$ & $<20$ & 150 & 500 & 1,500 & $>2,000$ \\
\hline LH0117C & 30 & $>2,000$ & 200 & $\mathbf{N}$ & $\mathbf{N}$ & $<20$ & 700 & 500 & N & $>2,000$ \\
\hline LHO118C & 50 & 700 & 300 & N & $\mathbf{N}$ & 70 & 50 & 300 & 700 & $>2,000$ \\
\hline LH0119C & 50 & $>2,000$ & 700 & 200 & $N$ & 300 & 200 & 500 & $N$ & $>2,000$ \\
\hline LHO120C & 50 & 1,500 & 200 & $N$ & $N$ & 150 & 300 & 300 & 700 & $>2,000$ \\
\hline LHO121C & 150 & $>5,000$ & $<500$ & 500 & N & $<50$ & N & 1,500 & $\mathbf{N}$ & $>5,000$ \\
\hline LHO122C & 70 & $>2,000$ & 700 & 700 & $<1,000$ & $<20$ & 3,000 & 700 & $\mathbf{N}$ & $>2,000$ \\
\hline LHO123C & 20 & $>2,000$ & $<200$ & $\mathbf{N}$ & N & N & 200 & 1,500 & $\mathbf{N}$ & $>2,000$ \\
\hline LH0124C & 20 & 1,500 & $<200$ & $N$ & $N$ & $<20$ & 150 & 2,000 & $N$ & $>2,000$ \\
\hline LH0125C & 70 & 300 & 500 & N & N & N & 150 & 1,500 & N & $>2,000$ \\
\hline LH0126C & 70 & $>2,000$ & 200 & 1,500 & 1,000 & 50 & 3,000 & 700 & N & $>2,000$ \\
\hline LHO127C & $<10$ & 20 & 3,000 & $N$ & $N$ & 100 & 150 & 200 & N & $>2,000$ \\
\hline LHO128C & 10 & 30 & 1,000 & N & N & 100 & 150 & 300 & $\mathbf{N}$ & $>2,000$ \\
\hline LH0129C & 50 & 150 & N & 2,000 & 3,000 & 50 & 3,000 & 1,000 & N & $>2,000$ \\
\hline LHO130C & 10 & $\mathbf{N}$ & 5,000 & N & N & 50 & 70 & 150 & 1,000 & $>2,000$ \\
\hline LH0131C & $\mathbf{N}$ & $N$ & 7,000 & N & N & 20 & 50 & 100 & N & $>2,000$ \\
\hline LH0132C & 70 & 700 & 1,000 & N & N & 100 & 300 & 1,000 & N & $>2,000$ \\
\hline LH0133C & 20 & $>2,000$ & 3,000 & N & N & 70 & 1,000 & 300 & $N$ & $>2,000$ \\
\hline LHO134C & 50 & $>2,000$ & N & 1,500 & 5,000 & N & 1,000 & 1,000 & N & $>2,000$ \\
\hline LH0135C & 15 & 1,000 & $\mathbf{N}$ & 1,500 & 1,500 & 50 & $>20,000$ & 300 & 3,000 & $>2,000$ \\
\hline LH0136C & 100 & 1,000 & $N$ & $>5,000$ & 7,000 & 50 & $>20,000$ & 1,000 & N & $>2,000$ \\
\hline LHO137C & 20 & 1,500 & N & N & N & 200 & 10,000 & 200 & 2,000 & $>2,000$ \\
\hline LHO138C & 150 & 70 & 200 & 200 & $N$ & 1,000 & N & 200 & $N$ & $>2,000$ \\
\hline LH0139C & 15 & N & $<200$ & N & N & 50 & $N$ & 70 & N & 500 \\
\hline LHO14OC & 150 & 1,500 & $<200$ & 3,000 & 5,000 & 50 & 150 & 3,000 & 3,000 & $>2,000$ \\
\hline LHO141C & 150 & 500 & $N$ & 3,000 & 5,000 & $N$ & 150 & 2,000 & N & $>2,000$ \\
\hline LH0142C & 150 & 700 & N & 5,000 & 5,000 & N & 150 & 2,000 & $\mathbf{N}$ & $>2,000$ \\
\hline LH0143C & 200 & 700 & $N$ & 3,000 & 5,000 & $N$ & 300 & $>5,000$ & $N$ & $>2,000$ \\
\hline LHO144C & 200 & 1,500 & $\mathbf{N}$ & 2,000 & 3,000 & $N$ & 70 & $>5,000$ & N & $>2,000$ \\
\hline LH0145C & 150 & 200 & $\mathbf{N}$ & 1,000 & 1,000 & 70 & 150 & 1,500 & 1,500 & $>2,000$ \\
\hline LH0146C & 70 & 700 & 300 & 500 & N & $<20$ & 1,000 & 700 & N & $>2,000$ \\
\hline LH0147C & 100 & 300 & 700 & 700 & $<1,000$ & 100 & 70 & 700 & 1,500 & $>2,000$ \\
\hline LH0152C & 70 & 700 & $<200$ & 1,500 & 1,000 & 1,000 & 300 & 1,500 & N & $>2,000$ \\
\hline LH0153C & 30 & 500 & 300 & 500 & N & 150 & 1,500 & 500 & N & $>2,000$ \\
\hline LH0154C & 200 & 70 & 200 & $\mathbf{N}$ & $\mathbf{N}$ & 100 & 50 & 500 & 2,000 & $>2,000$ \\
\hline LH0155C & 30 & 150 & $N$ & N & $\mathbf{N}$ & $<20$ & N & 300 & 3,000 & $>2,000$ \\
\hline LH0156C & 30 & 1,500 & N & 200 & N & 30 & 200 & 500 & N & $>2,000$ \\
\hline LHO157C & 100 & 30 & N & $N$ & $N$ & $\mathbf{N}$ & $\mathbf{N}$ & 1,000 & $N$ & $>2,000$ \\
\hline LHO158C & 70 & 1,500 & $<200$ & $N$ & $N$ & 70 & 150 & 300 & 700 & $>2,000$ \\
\hline LH0159C & 100 & 150 & 200 & $N$ & $N$ & 150 & 200 & 500 & $N$ & $>2,000$ \\
\hline LH0160C & 70 & 150 & 200 & N & $\mathbf{N}$ & 150 & 100 & 300 & 3,000 & $>2,000$ \\
\hline
\end{tabular}




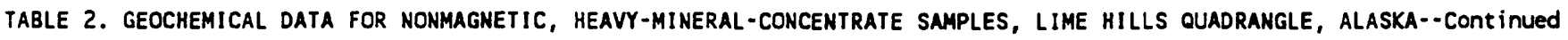

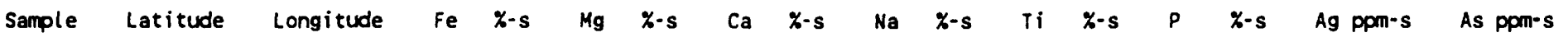

\begin{tabular}{|c|c|c|c|c|c|c|c|c|c|c|c|}
\hline LHO161C & 612440 & 153 & 2545 & 10.00 & .50 & 15.00 & 1.0 & 1.000 & .5 & 15.0 & 3,000 \\
\hline LH0162C & 61202 & 153 & 4128 & 7.00 & .10 & 15.00 & $<.5$ & .300 & 1.0 & 100.0 & 7,000 \\
\hline LH0163C & 611920 & 153 & 3552 & 3.00 & .10 & 20.00 & $<.5$ & .500 & .7 & 15.0 & 5,000 \\
\hline LH0164C & 611927 & 153 & 3549 & 7.00 & .10 & 15.00 & 1.0 & $>2.000$ & 3.0 & 300.0 & 500 \\
\hline LH0165C & 611711 & 153 & 4036 & .50 & .10 & 15.00 & .7 & 1.000 & 1.0 & $N$ & $N$ \\
\hline LHO166C & 614347 & 153 & 1338 & 5.00 & .30 & 20.00 & .7 & 2.000 & 5.0 & 15.0 & $<500$ \\
\hline LH0167C & 61359 & 153 & 179 & 3.00 & .07 & 30.00 & $<.5$ & 1.500 & 20.0 & 30.0 & 5,000 \\
\hline LH0168C & 613732 & 153 & 1456 & 1.00 & .30 & 30.00 & .7 & 2.000 & 20.0 & N & 500 \\
\hline LH0169C & 61391 & 153 & 1339 & .20 & .05 & 50.00 & .7 & .300 & 1.5 & 10.0 & N \\
\hline LH0170C & 61445 & 153 & 746 & 3.00 & .70 & 15.00 & 1.5 & .500 & 10.0 & 3.0 & N \\
\hline LHO171C & 614648 & 153 & 53 & 5.00 & .30 & 15.00 & 1.0 & 1.500 & 7.0 & 50.0 & 2,000 \\
\hline LH0172C & 614848 & 153 & 453 & 5.00 & .20 & 15.00 & .7 & $>2.000$ & 7.0 & 20.0 & $<500$ \\
\hline LHOIT3C & 614550 & 153 & 948 & 2.00 & .20 & 15.00 & 1.0 & 1.500 & 7.0 & 150.0 & 5,000 \\
\hline LH0174C & 614614 & 153 & 1133 & 1.00 & .50 & 15.00 & 1.0 & $>2.000$ & 10.0 & 2.0 & N \\
\hline LH0175C & 614757 & 153 & 1343 & 2.00 & .50 & 7.00 & .7 & 2.000 & 5.0 & 2.0 & N \\
\hline LH0200C & 614237 & 153 & 4733 & 2.00 & .10 & 7.00 & .7 & $>2.000$ & 3.0 & $N$ & $N$ \\
\hline LH0201C & 614144 & 153 & 4532 & 1.00 & .10 & 50.00 & $<.5$ & .070 & $>20.0$ & $N$ & $N$ \\
\hline LH0202C & 614031 & 153 & 4140 & 2.00 & .10 & 10.00 & .7 & $>2.000$ & 7.0 & 150.0 & $N$ \\
\hline LH0203C & 613939 & 153 & 4235 & 5.00 & .07 & 20.00 & 1.0 & .500 & 7.0 & 10.0 & 500 \\
\hline LH0204C & 614049 & 153 & 411 & 2.00 & .15 & 15.00 & .5 & $>2.000$ & 7.0 & 100.0 & N \\
\hline LH0205C & 614225 & 153 & 3556 & .70 & .07 & 7.00 & $<.5$ & 2.000 & 1.5 & 20.0 & $N$ \\
\hline LH0208C & 613946 & 153 & 3124 & 1.50 & .10 & 7.00 & .5 & $>2.000$ & 5.0 & 70.0 & 700 \\
\hline LHO209C & 613850 & 153 & 3159 & 2.00 & .15 & .50 & $<.5$ & .300 & $<.5$ & 70.0 & 1,500 \\
\hline LHO210C & 613720 & 153 & 3326 & 10.00 & .15 & 3.00 & 1.0 & $>2.000$ & 3.0 & 100.0 & $>20,000$ \\
\hline LHO211C & 613720 & 153 & 3337 & .70 & .10 & 1.50 & $<.5$ & .300 & 1.5 & 30.0 & 1,000 \\
\hline LHO212C & 61363 & 153 & 3642 & 1.50 & .07 & 1.50 & $<.5$ & .500 & 1.5 & N & H \\
\hline LHO213C & 613626 & 153 & 3058 & 30.00 & .20 & .20 & $<.5$ & .300 & $N$ & 150.0 & $>20,000$ \\
\hline LHO214C & 613621 & 153 & 3046 & 20.00 & .05 & 2.00 & $<.5$ & 1.500 & 3.0 & 200.0 & $>20,000$ \\
\hline LH0215C & 613257 & 153 & 3630 & 7.00 & .50 & 10.00 & 1.5 & .500 & 1.5 & 200.0 & $<500$ \\
\hline LH0216C & 61337 & 153 & 3810 & 1.50 & .20 & .30 & .5 & .700 & $N$ & N & N \\
\hline LH0217C & 613253 & 153 & 3417 & 3.00 & .15 & 30.00 & .5 & .200 & 7.0 & 150.0 & 1,000 \\
\hline LH0218C & 613315 & 153 & 3054 & 15.00 & .10 & 15.00 & .7 & 2.000 & 5.0 & 50.0 & 1,000 \\
\hline LH0219C & 613143 & 153 & 2924 & 15.00 & .70 & 5.00 & 1.0 & $>2.000$ & 1.0 & 30.0 & N \\
\hline LH0220C & 61313 & 153 & 2718 & 5.00 & .15 & 10.00 & .7 & $>2.000$ & 7.0 & 15.0 & 5,000 \\
\hline LH0221C & 613646 & 153 & 2510 & 2.00 & .15 & 7.00 & .7 & $>2.000$ & 5.0 & N & N \\
\hline LH0222C & 612937 & 153 & 2943 & 7.00 & .10 & 10.00 & .5 & $>2.000$ & 5.0 & 70.0 & 10,000 \\
\hline LHO223C & 61305 & 153 & 2236 & 20.00 & .07 & 3.00 & .5 & 1.000 & 1.5 & 100.0 & $>20,000$ \\
\hline LHO224C & 612825 & 153 & 230 & 7.00 & .07 & 3.00 & .7 & $>2.000$ & 2.0 & 30.0 & 1,000 \\
\hline LH0225C & 613052 & 153 & 2214 & 15.00 & .30 & 10.00 & .5 & 1.500 & 2.0 & 50.0 & 10,000 \\
\hline LH0226C & 613033 & 153 & 182 & 5.00 & .20 & 7.00 & 1.0 & 2.000 & 3.0 & N & $N$ \\
\hline LH0227C & 613320 & 153 & 1852 & 7.00 & .05 & 10.00 & .7 & .300 & 7.0 & 300.0 & $>20,000$ \\
\hline LHO228C & 613327 & 153 & 2110 & .50 & .15 & 50.00 & .5 & .200 & $>20.0$ & $N$ & N \\
\hline LH0229C & 613916 & 153 & 224 & 5.00 & .50 & 15.00 & 1.0 & 2.000 & 3.0 & 300.0 & 1,500 \\
\hline LHO230C & 613934 & 153 & 1612 & 10.00 & 3.00 & 10.00 & 1.0 & 2.000 & 3.0 & 50.0 & 1,000 \\
\hline LHO231C & 613745 & 153 & 414 & 1.50 & .30 & 10.00 & 1.0 & 2.000 & 3.0 & 20.0 & 1,500 \\
\hline LH0232C & 613859 & 153 & 442 & 1.50 & .20 & 5.00 & .7 & 1.500 & 3.0 & 50.0 & 1,500 \\
\hline LH0234C & 613657 & 153 & 1233 & 10.00 & .30 & 10.00 & .5 & 2.000 & 3.0 & 300.0 & $>20,000$ \\
\hline LнО235C & 613658 & 153 & 1225 & 15.00 & .30 & 3.00 & .7 & 1.500 & 2.0 & 50.0 & $>20,000$ \\
\hline LH0236C & 61384 & 153 & 1118 & 1.50 & .07 & 50.00 & .5 & .300 & $N$ & 100.0 & 2,000 \\
\hline LH0237C & 614122 & 153 & 326 & 1.00 & .10 & 10.00 & 1.5 & 1.000 & 7.0 & 15.0 & 3,000 \\
\hline
\end{tabular}


TABLE 2. GEOCHEMICAL DATA FOR NONMAGNETIC, heAVY-MINERAL-CONCENTRATE SAMPLES, LIME hILLS QUADRANGLE, ALASKA--ContinUed

Sample AU ppm-s B ppm-s Ba ppm-s Be ppm-s $\quad$ Bi ppm-s Cd ppm-s Co ppm-s Cr ppm-s CU ppm-s

\begin{tabular}{|c|c|c|c|c|c|c|c|c|c|}
\hline LH0161C & N & 20 & 7,000 & 3 & 20 & N & 70 & 20 & 300 \\
\hline LH0162C & $N$ & $N$ & $>10,000$ & 3 & 100 & 150 & 50 & $<20$ & 30 \\
\hline LH0163C & N & $N$ & 150 & 5 & 30 & 50 & 70 & $<20$ & 30 \\
\hline LH0164C & $N$ & $N$ & $>10,000$ & 15 & 200 & 150 & $<20$ & $<20$ & 300 \\
\hline LH0165C & N & $N$ & 300 & 2 & 200 & N & $N$ & 30 & 20 \\
\hline LH0166C & $N$ & 300 & 5,000 & 5 & 30 & N & 30 & 30 & 70 \\
\hline LHO167C & $N$ & N & 3,000 & $N$ & 30 & 70 & 70 & 50 & 30 \\
\hline LH0168C & N & $N$ & 300 & $N$ & $N$ & N & $<20$ & 50 & 100 \\
\hline LH0169C & $N$ & N & 50 & 30 & 700 & N & N & $<20$ & $<10$ \\
\hline LH0170C & $N$ & 50 & 300 & 3 & 200 & N & $<20$ & 20 & 30 \\
\hline LHO171C & 50 & 700 & 1.500 & 3 & 700 & N & 150 & 50 & 1,000 \\
\hline LH0172C & $N$ & 700 & 7,000 & 2 & 20 & $N$ & 20 & 100 & 150 \\
\hline LHO173C & 20 & 20 & 500 & 2 & 150 & N & $<20$ & 30 & 150 \\
\hline LH0174C & $<20$ & 1,500 & 500 & $N$ & N & N & N & 500 & 100 \\
\hline LH0175C & N & 70 & 5,000 & $N$ & 50 & N & $<20$ & 200 & 30 \\
\hline LH0200C & N & $N$ & 500 & $\mathbf{N}$ & $\mathbf{N}$ & N & 50 & $<20$ & 20 \\
\hline LH0201C & N & $N$ & 70 & $\mathbf{N}$ & $\mathbf{N}$ & N & 70 & $<20$ & $<10$ \\
\hline LH0202C & N & 20 & $>10,000$ & $N$ & $<20$ & N & $<20$ & 200 & 100 \\
\hline LH0203C & N & $N$ & 1.500 & N & 30 & N & 100 & $<20$ & 50 \\
\hline LH0204C & 70 & 70 & $>10,000$ & $N$ & N & N & $<20$ & 70 & 70 \\
\hline LH0205C & $N$ & $N$ & $>10,000$ & N & 70 & 100 & $<20$ & 70 & 500 \\
\hline LH0208C & N & 20 & $>10,000$ & 2 & 30 & $N$ & $<20$ & 70 & 150 \\
\hline LH0209C & $N$ & 30 & $>10,000$ & 2 & 70 & N & $<20$ & 150 & 20 \\
\hline LH0210C & 70 & 1,000 & $>10,000$ & N & 1.500 & N & 700 & 70 & 700 \\
\hline LH0211C & $N$ & 50 & 1,500 & 2 & 150 & N & 70 & 100 & 30 \\
\hline LH0212C & $N$ & 20 & 500 & N & N & N & $<20$ & 150 & 50 \\
\hline LH0213C & $N$ & 20 & 10,000 & $N$ & N & N & 100 & $<20$ & 300 \\
\hline LH0214C & N & 20 & $>10,000$ & N & N & N & 70 & 30 & 200 \\
\hline LH0215C & $N$ & 300 & 2,000 & 3 & $>2,000$ & N & 30 & 30 & 10,000 \\
\hline LH0216C & $N$ & 20 & 3,000 & 3 & N & N & N & 200 & 50 \\
\hline LH0217C & $N$ & 30 & $>10,000$ & 5 & $>2,000$ & $N$ & 100 & $<20$ & 70 \\
\hline LH0218C & $N$ & 50 & 3.000 & $N$ & 700 & $N$ & 700 & 30 & 50 \\
\hline LHO219C & $N$ & 2,000 & 5,000 & $N$ & 300 & N & 300 & 70 & 100 \\
\hline LHO220C & $N$ & 150 & 7,000 & 3 & 700 & $N$ & 70 & 150 & 100 \\
\hline LH0221C & $N$ & 20 & $>10,000$ & 3 & N & $N$ & 20 & 150 & 100 \\
\hline LHO222C & $<20$ & 50 & $>10,000$ & 3 & 700 & N & 200 & 100 & 100 \\
\hline LH0223C & 20 & $N$ & $>10,000$ & $N$ & 100 & $N$ & 200 & $<20$ & 150 \\
\hline LH0224C & $\mathbf{N}$ & N & 3,000 & 3 & $N$ & N & 70 & $<20$ & 100 \\
\hline LHO225C & $N$ & $N$ & $>10,000$ & 3 & 70 & N & 200 & 20 & 200 \\
\hline LH0226C & $N$ & $N$ & 2,000 & 5 & 500 & N & N & 30 & 100 \\
\hline LH0227C & $N$ & N & 10,000 & $N$ & 1,500 & $N$ & 700 & $<20$ & 100 \\
\hline LH0228C & $N$ & 20 & 1.000 & N & N & N & 30 & $<20$ & 30 \\
\hline LHO229C & $N$ & $N$ & 5.000 & 3 & 300 & $N$ & 20 & 150 & 700 \\
\hline LHO230C & N & $N$ & 1,000 & 5 & 1,500 & $N$ & 30 & 50 & 500 \\
\hline LH0231C & $N$ & 150 & 300 & 7 & 150 & N & 30 & 20 & 200 \\
\hline LH0232C & $N$ & 70 & 300 & 3 & 500 & $N$ & N & 20 & 300 \\
\hline LH0234C & 70 & $N$ & 3,000 & N & 500 & $N$ & 300 & 20 & 1,000 \\
\hline LH0235C & N & $N$ & 3,000 & N & 1,500 & $N$ & 700 & 20 & 2,000 \\
\hline LH0236C & $N$ & $N$ & 100 & 70 & 500 & N & N & $<20$ & 30 \\
\hline LH0237C & N & 50 & 300 & 3 & 500 & $N$ & 30 & 30 & 100 \\
\hline
\end{tabular}




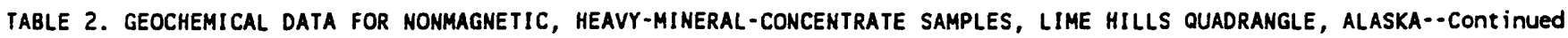

Sample Ga ppm-s Ge ppm-s La ppm-s Mn ppm-s Mo ppm-s Nb ppm-s Ni ppm-s Pb ppm-s Pt ppm-s Sb ppm-s

\begin{tabular}{|c|c|c|c|c|c|c|c|c|c|c|}
\hline LH0161C & 30 & $\mathbf{N}$ & 150 & 1,500 & $\mathbf{N}$ & $<50$ & 20 & 200 & $\mathbf{N}$ & N \\
\hline LH0162C & 20 & $\mathbf{N}$ & $\mathbf{N}$ & 500 & 100 & $<50$ & $<10$ & 1,500 & $\mathbf{N}$ & N \\
\hline LH0163C & 30 & $\mathbf{N}$ & $N$ & 1,000 & 50 & $<50$ & $<10$ & 2,000 & $\mathbf{N}$ & N \\
\hline LH0164C & 10 & N & 300 & 700 & 30 & 100 & $<10$ & 15,000 & $N$ & $<200$ \\
\hline LH0165C & 10 & $N$ & 1,500 & 200 & 150 & 100 & $\mathbf{N}$ & 200 & N & N \\
\hline LH0166C & 15 & $N$ & 500 & 1,000 & 100 & 50 & 30 & 5,000 & $\mathbf{N}$ & N \\
\hline LH0167C & $\mathbf{N}$ & $N$ & 1,000 & 700 & $N$ & $<50$ & 20 & 7,000 & $\mathbf{N}$ & N \\
\hline LH0168C & $<10$ & $\mathbf{N}$ & 700 & 1,000 & 20 & 50 & $<10$ & 300 & $\mathbf{N}$ & $N$ \\
\hline LH0169C & $<10$ & $\mathbf{N}$ & 500 & 300 & 700 & 150 & N & 100 & $\mathbf{N}$ & N \\
\hline LH0170C & 15 & $\mathbf{N}$ & 700 & 1,500 & 700 & $<50$ & $<10$ & 3,000 & $\mathbf{N}$ & N \\
\hline LH0171C & 20 & $N$ & 700 & 1,000 & 150 & 50 & 70 & 7,000 & $\mathbf{N}$ & N \\
\hline LH0172C & 10 & $\mathbf{N}$ & 300 & 1,000 & 15 & 50 & 10 & 3,000 & $\mathbf{N}$ & 300 \\
\hline LH0173C & 15 & $N$ & 300 & 1,000 & 500 & 50 & 15 & $>50,000$ & N & N \\
\hline LHO174C & 15 & $\mathbf{N}$ & 300 & 500 & $\mathbf{N}$ & 50 & $<10$ & 20 & N & N \\
\hline LHO175C & 20 & $\mathbf{N}$ & 300 & 700 & 20 & $<50$ & $<10$ & 15 & $N$ & N \\
\hline LH0200C & $<10$ & $\mathbf{N}$ & 300 & 500 & 30 & $<50$ & $<10$ & 50 & $N$ & N \\
\hline LH0201C & $N$ & $\mathbf{N}$ & 700 & 700 & $N$ & N & $<10$ & N & $\mathbf{N}$ & N \\
\hline LH0202C & $<10$ & $\mathbf{N}$ & 300 & 300 & $\mathbf{N}$ & 70 & 30 & 5,000 & $\mathbf{N}$ & N \\
\hline LHO2O3C & $<10$ & $N$ & 500 & 500 & 15 & $N$ & 15 & 300 & $\mathbf{N}$ & $N$ \\
\hline LH0204C & $<10$ & N & 500 & 1,000 & $\mathbf{N}$ & 100 & 20 & 500 & $\mathbf{N}$ & 200 \\
\hline LH0205C & $<10$ & $\mathbf{N}$ & $\mathbf{N}$ & 150 & $\mathbf{N}$ & 70 & 10 & 1,000 & $\mathbf{N}$ & $<200$ \\
\hline LH0208C & $<10$ & N & 150 & 150 & $\mathbf{N}$ & 100 & 10 & 15,000 & $N$ & N \\
\hline LH0209C & 50 & $\mathbf{N}$ & N & 100 & $\mathbf{N}$ & $<50$ & 15 & 1,000 & $N$ & N \\
\hline LHO210C & $<10$ & $N$ & 150 & 150 & $N$ & 50 & 700 & 5,000 & N & $<200$ \\
\hline LH0211C & 30 & $\mathbf{N}$ & 150 & 150 & N & $<50$ & 50 & 1,500 & $\mathbf{N}$ & $\mathbf{N}$ \\
\hline LH0212C & 30 & $\mathbf{N}$ & 100 & 150 & $\mathbf{N}$ & $<50$ & $<10$ & 70 & $\mathbf{N}$ & N \\
\hline LH0213C & $\mathbf{N}$ & $\mathbf{N}$ & $\mathbf{N}$ & 100 & $\mathbf{N}$ & $<50$ & 300 & 2,000 & $\mathbf{N}$ & 300 \\
\hline LH0214C & $<10$ & $N$ & 100 & 150 & N & $<50$ & 150 & 7,000 & $N$ & 300 \\
\hline LHO215C & 50 & $\mathbf{N}$ & 100 & 1,000 & 1,500 & $<50$ & 30 & 15,000 & $\mathbf{N}$ & N \\
\hline LH0216C & 50 & $\mathbf{N}$ & $N$ & 100 & N & $<50$ & $<10$ & 500 & $N$ & N \\
\hline LH0217C & 70 & $N$ & 300 & 300 & 50 & $<50$ & $<10$ & 7,000 & $N$ & $N$ \\
\hline LH0218C & $<10$ & $\mathbf{N}$ & 500 & 200 & $N$ & $<50$ & 200 & 200 & $N$ & N \\
\hline LHO219C & 20 & $\mathbf{N}$ & $N$ & 150 & $N$ & 70 & 70 & 500 & $\mathbf{N}$ & 200 \\
\hline LH0220C & 15 & $\mathbf{N}$ & 200 & 500 & 30 & 150 & 20 & 1,000 & N & N \\
\hline LHO221C & 15 & $N$ & 300 & 300 & $N$ & 150 & 10 & 700 & $N$ & N \\
\hline LHO222C & 10 & $N$ & 500 & 300 & N & 100 & 30 & 2,000 & $\mathbf{N}$ & N \\
\hline LH0223C & $<10$ & $\mathbf{N}$ & 150 & 150 & N & 50 & 70 & 30,000 & $\mathbf{N}$ & $<200$ \\
\hline LHO224C & 10 & $N$ & 150 & 300 & $\mathbf{N}$ & $<50$ & 20 & 1,500 & N & $N$ \\
\hline LH0225C & 15 & $\mathbf{N}$ & 150 & 500 & N & $<50$ & 30 & 5,000 & N & $N$ \\
\hline LH0226C & 20 & 30 & 300 & 1,500 & 300 & 70 & N & 1,500 & $\mathbf{N}$ & $N$ \\
\hline LH0227C & $\mathbf{N}$ & $N$ & 300 & 500 & 300 & $<50$ & 200 & 7,000 & $\mathbf{N}$ & $<200$ \\
\hline LHO228C & $\mathbf{N}$ & $N$ & 1,500 & 700 & $N$ & $\mathbf{N}$ & $<10$ & 300 & $\mathbf{N}$ & $\mathbf{N}$ \\
\hline LH0229C & 20 & $N$ & 300 & 1,000 & 200 & 50 & $<10$ & 7,000 & $N$ & N \\
\hline LHO23OC & 20 & $N$ & 700 & 3,000 & $\mathbf{N}$ & 70 & $<10$ & 2,000 & $N$ & N \\
\hline LH0231C & 10 & $N$ & 700 & 700 & 100 & 70 & $N$ & 2,000 & $N$ & N \\
\hline LH0232C & $<10$ & $N$ & 700 & 500 & 700 & 50 & $\mathbf{N}$ & 7,000 & N & N \\
\hline LHO234C & $<10$ & $\mathbf{N}$ & 300 & 500 & 500 & $<50$ & 30 & 30,000 & N & $<200$ \\
\hline LH0235C & 10 & $\mathbf{N}$ & 150 & 200 & $\mathbf{N}$ & $<50$ & 100 & 1,500 & N & $\mathbf{N}$ \\
\hline LH0236C & $<10$ & $\mathbf{N}$ & 300 & 300 & $N$ & 100 & $\mathbf{N}$ & 5,000 & $\mathbf{N}$ & N \\
\hline LH0237C & 10 & $\mathbf{N}$ & 500 & 1,000 & 1,000 & 50 & $<10$ & 3,000 & N & N \\
\hline
\end{tabular}


TABLE 2. GEOChEMICAL DATA FOR NONMAGNETIC, hEAVY-MINERAL-CONCENTRATE SAMPLES, LIME hILLS QUADRANGLE, ALASKA--CONT InUEd

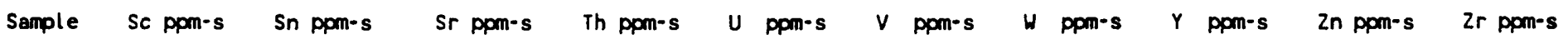

\begin{tabular}{|c|c|c|c|c|c|c|c|c|c|c|}
\hline LHO161C & 30 & 100 & 700 & $\mathbf{N}$ & N & 300 & 150 & 150 & $N$ & $>2,000$ \\
\hline LH0162C & 50 & 150 & 200 & $N$ & $N$ & 70 & 10,000 & 500 & 700 & $>2,000$ \\
\hline LH0163C & 20 & 200 & $<200$ & $N$ & $N$ & 100 & 1,000 & 200 & N & $>2,000$ \\
\hline LH0164C & 70 & 100 & 1,000 & N & N & 30 & 1,000 & 500 & 3,000 & $>2,000$ \\
\hline LH0165C & 70 & N & $<200$ & $>5,000$ & 15,000 & 70 & 20,000 & 1,000 & N & $>2,000$ \\
\hline LH0166C & 20 & 1,000 & 500 & N & $N$ & 150 & 2,000 & 700 & N & $>2,000$ \\
\hline LHO167C & 50 & $>2,000$ & 300 & 200 & $N$ & 100 & 500 & 700 & $N$ & $>2,000$ \\
\hline LH0168C & 20 & 1,000 & 500 & $N$ & $N$ & 100 & 70 & 500 & $N$ & $>2,000$ \\
\hline LHO169C & 15 & $>2,000$ & 500 & 300 & N & N & N & 5,000 & $N$ & $>2,000$ \\
\hline LHO170C & 20 & $>2,000$ & 700 & 1,500 & 1,000 & 70 & 3,000 & 500 & $N$ & $>2,000$ \\
\hline LH0171C & 30 & $>2,000$ & 500 & 700 & N & 100 & 15,000 & 500 & $N$ & $>2,000$ \\
\hline LH0172C & 30 & $>2,000$ & 700 & $N$ & $N$ & 150 & 150 & 300 & $N$ & $>2,000$ \\
\hline LH0173C & 30 & 1,500 & 500 & $\mathbf{N}$ & $N$ & 150 & 5,000 & 300 & $N$ & $>2,000$ \\
\hline LH0174C & 70 & 150 & 200 & $N$ & N & 300 & 200 & 200 & $\mathbf{N}$ & $>2,000$ \\
\hline LH0175C & 30 & 700 & 200 & $\mathbf{N}$ & N & 150 & 200 & 300 & $N$ & $>2,000$ \\
\hline LHO200C & 50 & 150 & $<200$ & 1,500 & 1,500 & 200 & 300 & 700 & $\mathbf{N}$ & $>2,000$ \\
\hline LH0201C & $N$ & $N$ & 1,500 & N & N & 100 & 50 & 700 & $\mathbf{N}$ & $>2,000$ \\
\hline LH0202C & 70 & N & 3,000 & $N$ & N & 100 & 50 & 300 & N & $>2,000$ \\
\hline LH0203C & 50 & 70 & 500 & 300 & $N$ & 50 & 50 & 700 & N & $>2,000$ \\
\hline LH0204C & 50 & $N$ & 3,000 & N & $N$ & 150 & 150 & 300 & N & $>2,000$ \\
\hline LH0205C & 15 & 30 & 3,000 & $N$ & $N$ & 70 & 70 & 150 & 3,000 & $>2,000$ \\
\hline LH0208C & 30 & $>2,000$ & 2,000 & N & $\mathbf{N}$ & 100 & 20,000 & 200 & 500 & $>2,000$ \\
\hline LH0209C & $N$ & 150 & 200 & N & $N$ & 150 & 100 & 30 & N & $>2,000$ \\
\hline LHO210C & 30 & 1,000 & 1,000 & N & $N$ & 150 & 500 & 200 & N & $>2,000$ \\
\hline LH0211C & 10 & 700 & $<200$ & N & $N$ & 150 & 700 & 150 & $\mathbf{N}$ & $>2,000$ \\
\hline LH0212C & N & $N$ & $<200$ & N & N & 150 & $N$ & 100 & N & $>2,000$ \\
\hline LH0213C & $<10$ & N & N & N & N & 30 & $N$ & 30 & 1,500 & 700 \\
\hline LHO214C & $<10$ & $<20$ & 1,000 & N & N & 70 & N & 100 & N & $>2,000$ \\
\hline LH0215C & 30 & $N$ & 700 & N & $N$ & 300 & N & 100 & $\mathbf{N}$ & $>2,000$ \\
\hline LHO216C & $<10$ & $<20$ & $<200$ & $N$ & N & 200 & $N$ & 30 & N & $>2,000$ \\
\hline LHO217C & $N$ & 30 & 500 & $N$ & $N$ & 200 & 70 & 300 & 700 & 1,500 \\
\hline LH0218C & 50 & 50 & 200 & $N$ & $N$ & 150 & 70 & 700 & N & $>2,000$ \\
\hline LH0219C & 30 & 300 & $N$ & $N$ & $N$ & 200 & 200 & 200 & N & 2,000 \\
\hline LH0220C & 50 & 2,000 & 500 & $N$ & N & 200 & 15,000 & 300 & N & $>2,000$ \\
\hline LH0221C & 30 & 30 & 1,000 & $N$ & $N$ & 150 & N & 300 & N & $>2,000$ \\
\hline LH0222C & 70 & $>2,000$ & 500 & N & N & 150 & 1,000 & 500 & $\mathbf{N}$ & $>2,000$ \\
\hline LH0223C & 20 & 1,500 & N & N & N & 50 & 10,000 & 300 & $N$ & $>2,000$ \\
\hline LH0224C & 70 & 500 & $<200$ & N & N & 70 & N & 700 & $N$ & $>2,000$ \\
\hline LH0225C & 20 & $>2,000$ & 500 & N & N & 100 & N & 200 & 500 & $>2,000$ \\
\hline LH0226C & 100 & 1,000 & 200 & N & N & 100 & N & 500 & $N$ & $>2,000$ \\
\hline LH0227C & 70 & 1,500 & 300 & 1,000 & 1,500 & $<20$ & 5,000 & 700 & 700 & $>2,000$ \\
\hline LHO228C & 50 & 500 & 1,500 & N & N & 70 & 300 & 700 & N & $>2,000$ \\
\hline LH0229C & 70 & $>2,000$ & 500 & $N$ & N & 150 & 300 & 300 & 1,500 & $>2,000$ \\
\hline LHO230C & 100 & 200 & 300 & N & N & 70 & N & 500 & 1,500 & $>2,000$ \\
\hline LHO231C & 100 & $>2,000$ & 200 & 1,000 & 1,500 & 70 & 700 & 1,000 & N & $>2,000$ \\
\hline LHO232C & 100 & $>2,000$ & $<200$ & 1,500 & 1,000 & 70 & 7,000 & 700 & $N$ & $>2,000$ \\
\hline LHO234C & 50 & 700 & 200 & N & N & 70 & 100 & 500 & 3,000 & $>2,000$ \\
\hline LH0235C & 20 & 500 & 200 & N & N & 70 & 200 & 300 & N & $>2,000$ \\
\hline LHO236C & 30 & $>2,000$ & 200 & 1,500 & 1,500 & 20 & 100 & $>5,000$ & 1,500 & $>2,000$ \\
\hline $2 \mathrm{HO} 0237 \mathrm{C}$ & 70 & $>2,000$ & 500 & 700 & N & 70 & 5,000 & 700 & N & $>2,000$ \\
\hline
\end{tabular}


TABLE 2. GEOCHEMICAL DATA FOR NONMAGNETIC, hEAVY-MINERAL-CONCENTRATE SAMPLES, LIME HILLS QUADRANGLE, ALASKA--CONTINUEd

Sample Latitude Longitude fe $\%$-s $\mathrm{Mg} \%$-s $\mathrm{Ca} \%$-s $\mathrm{Na} \%$-s $\quad \mathrm{Ti} \quad \%$-s $\quad \mathrm{P} \quad \%$-s Ag ppm-s As ppm-s

\begin{tabular}{|c|c|c|c|c|c|c|c|c|c|c|c|}
\hline LH0238C & 614346 & 153 & 559 & 1.00 & .20 & 10.00 & 1.5 & .700 & 7.0 & 2.0 & $N$ \\
\hline LH0239C & 614339 & 153 & 559 & 1.00 & .15 & 10.00 & 1.0 & 1.000 & 5.0 & 200.0 & 1,500 \\
\hline LH0242C & $6146 \quad 17$ & 153 & 3415 & 3.00 & .20 & 7.00 & .7 & 2.000 & 7.0 & N & N \\
\hline LH0243C & 614924 & 153 & 3233 & 15.00 & .15 & 2.00 & .5 & 1.500 & 1.5 & 300.0 & $>20,000$ \\
\hline LH0244C & 614747 & 153 & 2524 & 7.00 & .30 & 3.00 & .7 & $>2.000$ & 5.0 & 10.0 & 3,000 \\
\hline LH0245C & 614622 & 153 & 2849 & 5.00 & .20 & 3.00 & .7 & $>2.000$ & 3.0 & 5.0 & 700 \\
\hline LH0246C & 61522 & 153 & 1953 & 1.00 & .15 & 5.00 & 1.5 & .700 & 3.0 & 7.0 & $<500$ \\
\hline LH0247C & 615345 & 153 & 199 & 2.00 & .15 & 2.00 & 1.0 & $>2.000$ & 2.0 & 150.0 & $<500$ \\
\hline LH0248C & 615248 & 153 & 243 & 1.00 & .10 & 20.00 & 1.5 & .500 & 7.0 & N & $<500$ \\
\hline LHO250C & 61576 & 153 & 2532 & 10.00 & .50 & 1.50 & .7 & .500 & 1.5 & 5.0 & 1,500 \\
\hline LH0251C & $6157 \quad 10$ & 153 & 2535 & 7.00 & 1.00 & 2.00 & .7 & .700 & 2.0 & $N$ & $<500$ \\
\hline LH0252C & 615739 & 153 & 240 & 1.00 & .10 & 20.00 & .7 & .300 & 20.0 & $N$ & N \\
\hline LH0253C & 614533 & 153 & 2822 & 7.00 & .30 & 2.00 & .7 & 2.000 & 3.0 & $N$ & N \\
\hline LH0254C & 614058 & 153 & 2349 & 3.00 & .50 & 10.00 & .7 & 2.000 & 5.0 & $N$ & N \\
\hline LH0255C & 613954 & 153 & 2353 & 10.00 & 1.00 & 2.00 & .7 & 1.000 & 2.0 & 20.0 & 3,000 \\
\hline LH0256C & 61402 & 153 & 2357 & 3.00 & .10 & 2.00 & .5 & $>2.000$ & 3.0 & 100.0 & 1,500 \\
\hline LH0257C & 613852 & 153 & 4314 & .70 & .10 & 30.00 & .7 & .700 & $>20.0$ & $N$ & N \\
\hline LH0258C & 613817 & 153 & 4125 & 1.00 & .07 & 2.00 & .5 & 1.000 & 3.0 & 5.0 & $N$ \\
\hline LH0259C & 612721 & 153 & 19 & 7.00 & .20 & 1.50 & 1.0 & 2.000 & 2.0 & 3.0 & N \\
\hline LH0260C & 612727 & 153 & 15 & 5.00 & .10 & 5.00 & 1.0 & 2.000 & 3.0 & 100.0 & 1,500 \\
\hline LH0261C & 61268 & 153 & 738 & 15.00 & .05 & 2.00 & 1.0 & .700 & 2.0 & 50.0 & 3,000 \\
\hline LH0262C & 612615 & 153 & 753 & 7.00 & .20 & 3.00 & 1.0 & 2.000 & 2.0 & 20.0 & 1,000 \\
\hline LH0263C & 61284 & 153 & 755 & 5.00 & .15 & 3.00 & 1.0 & $>2.000$ & .7 & 70.0 & 500 \\
\hline LHO264C & 61258 & 153 & 817 & 7.00 & .15 & 2.00 & 1.0 & 2.000 & 1.5 & 100.0 & 1,000 \\
\hline LH0266C & 612552 & 153 & 1516 & 7.00 & .20 & 2.00 & 1.0 & 1.000 & 1.5 & 100.0 & 7,000 \\
\hline LH0267C & 612541 & 153 & 200 & 7.00 & .15 & 3.00 & 1.0 & 1.500 & 1.0 & 20.0 & 10,000 \\
\hline LH0268C & 612050 & 153 & 1828 & .70 & .05 & .50 & .5 & .150 & 1.0 & 5.0 & 5,000 \\
\hline LH0269C & 612153 & 153 & 181 & 2.00 & .05 & .50 & .5 & .200 & 2.0 & 10.0 & 20,000 \\
\hline LH0270C & 612351 & 153 & 175 & 7.00 & 1.50 & 3.00 & .7 & 1.500 & 1.0 & 50.0 & N \\
\hline LHO271C & 612451 & 153 & 1615 & 5.00 & .30 & 1.00 & .5 & .500 & 5.0 & 20.0 & N \\
\hline LH0272C & 612242 & 153 & 106 & .50 & .05 & .30 & 1.0 & .150 & .7 & $N$ & 700 \\
\hline LH0273C & 612053 & 153 & 455 & .50 & .10 & .70 & .5 & .200 & 1.0 & N & 1,500 \\
\hline LH0274C & 611958 & 153 & 634 & .50 & .07 & 3.00 & .7 & 1.000 & 3.0 & $N$ & 500 \\
\hline LH0275C & 61161 & 153 & 234 & .50 & .10 & 10.00 & 1.5 & .300 & 5.0 & $N$ & N \\
\hline LH0276C & 613310 & 153 & 1259 & 5.00 & .20 & 15.00 & 1.0 & 2.000 & 7.0 & 3.0 & 500 \\
\hline LH0279C & 691720 & 153 & 758 & 7.00 & 2.00 & 15.00 & 1.0 & 1.000 & 1.5 & $N$ & 1,500 \\
\hline LH0280C & 611619 & 153 & 1314 & 1.00 & .10 & 1.00 & .5 & .700 & 1.5 & 2.0 & $<500$ \\
\hline LH0281C & 611544 & 153 & 1539 & .20 & $<.05$ & .70 & .5 & 1.500 & .7 & N & N \\
\hline LH0282C & 611531 & 153 & 1732 & 2.00 & .07 & 7.00 & 1.0 & .300 & 3.0 & 100.0 & 2,000 \\
\hline LH0283C & 611136 & 153 & 209 & 5.00 & 1.00 & 10.00 & 1.0 & 2.000 & 7.0 & $N$ & N \\
\hline LH0284C & 61124 & 153 & 1734 & 7.00 & 2.00 & 5.00 & 1.0 & $>2.000$ & 1.5 & 15.0 & 500 \\
\hline LH0285C & 611121 & 153 & 1552 & 7.00 & .50 & 3.00 & .7 & $>2.000$ & 3.0 & 100.0 & 500 \\
\hline LH0286C & 61858 & 153 & 103 & 7.00 & 1.50 & 2.00 & 1.0 & 1.000 & 3.0 & 20.0 & 500 \\
\hline LH0287C & 611435 & 153 & 935 & 1.00 & .20 & 2.00 & $N$ & 1.000 & 3.0 & 5.0 & N \\
\hline LH0288C & 611336 & 153 & 420 & 5.00 & 1.00 & 3.00 & 1.0 & 1.000 & 1.0 & 50.0 & 500 \\
\hline LH0289C & 61929 & 153 & 656 & 5.00 & 1.00 & 2.00 & .5 & 2.000 & 5.0 & 50.0 & 7,000 \\
\hline LH0290С & 61332 & 153 & 213 & 1.00 & .10 & 5.00 & $<.5$ & $>2.000$ & 1.5 & $N$ & 500 \\
\hline LH0291C & 61552 & 153 & 842 & 1.00 & .05 & .70 & $N$ & .300 & 2.0 & $N$ & $N$ \\
\hline LH0292C & 6148 & 153 & 656 & 1.00 & .05 & .70 & $N$ & .300 & 2.0 & 5.0 & $N$ \\
\hline LH0293C & 61414 & 153 & 1257 & 1.50 & .05 & .70 & $N$ & .700 & 1.5 & $N$ & 700 \\
\hline
\end{tabular}




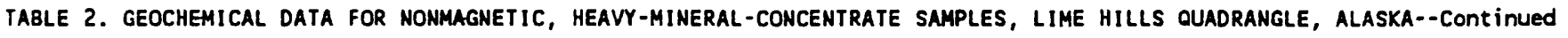

Sample Au ppm-s B ppm-s Ba ppm-s Be ppm-s Bi ppm-s lod ppm-s Co ppm-s Cr ppm-s Cu ppm-s

\begin{tabular}{|c|c|c|c|c|c|c|c|c|c|}
\hline LH0238C & N & 50 & 200 & 3 & $N$ & $N$ & $\mathbf{N}$ & 20 & 30 \\
\hline LH0239C & N & $N$ & 200 & 3 & 70 & $N$ & 20 & 30 & 100 \\
\hline LH0242C & N & 150 & 2,000 & 2 & $N$ & $N$ & 20 & 150 & 100 \\
\hline LH0243C & N & 100 & $>10,000$ & $N$ & 700 & $N$ & 100 & 70 & 500 \\
\hline LH0244C & N & 200 & 5,000 & 3 & N & $N$ & 50 & 200 & 300 \\
\hline LH0245C & N & 100 & 3,000 & 3 & $N$ & $N$ & 30 & 500 & 150 \\
\hline LH0246C & $N$ & 50 & 1,500 & 2 & 70 & $N$ & $<20$ & 30 & 30 \\
\hline LH0247C & $N$ & 20 & 1,500 & 5 & 50 & $N$ & $<20$ & 50 & 70 \\
\hline LH0248C & $N$ & 50 & 5,000 & $N$ & N & $N$ & $<20$ & 30 & 100 \\
\hline LHO250C & $\mathbf{N}$ & 700 & $>10,000$ & 3 & $\mathbf{N}$ & $N$ & 70 & 100 & 500 \\
\hline LH0251C & N & 200 & $>10,000$ & 2 & $N$ & $N$ & 30 & 100 & 150 \\
\hline LH0252C & N & 30 & 150 & $N$ & $N$ & N & N & 30 & 20 \\
\hline LH0253C & N & 100 & 5,000 & 3 & $\mathbf{N}$ & N & 50 & 500 & 150 \\
\hline LH0254C & $N$ & 200 & 1,500 & 3 & N & $N$ & 20 & 500 & 200 \\
\hline LH0255C & $N$ & 200 & 700 & 3 & 100 & $N$ & 70 & 200 & 500 \\
\hline LHO256C & $N$ & 20 & $>10,000$ & 2 & 50 & $N$ & 30 & 70 & 150 \\
\hline LH0257C & $N$ & $N$ & 150 & $N$ & $N$ & N & N & $<20$ & 50 \\
\hline LHO258C & $N$ & 20 & 2,000 & 3 & $N$ & $N$ & $<20$ & 100 & 50 \\
\hline LHO259C & N & $N$ & 7,000 & 7 & N & N & $<20$ & 20 & 50 \\
\hline LHO260C & $<20$ & $N$ & 700 & 3 & 20 & N & 20 & $<20$ & 100 \\
\hline LH0261C & $N$ & $N$ & $>10,000$ & 2 & 150 & 150 & 70 & $<20$ & 500 \\
\hline LH0262C & N & $N$ & 7,000 & 3 & 100 & 150 & 20 & $<20$ & 500 \\
\hline LH0263C & $N$ & $N$ & $>10,000$ & 3 & 150 & N & $<20$ & $<20$ & 50 \\
\hline LH0264C & N & $N$ & 3,000 & 3 & 500 & 100 & 30 & $<20$ & 700 \\
\hline LH0266C & N & N & 700 & 7 & 1,500 & $N$ & 30 & $<20$ & 2,000 \\
\hline LH0267C & N & N & 500 & 7 & 1,000 & $N$ & 20 & 20 & 1,000 \\
\hline LH0268C & $N$ & N & 150 & 3 & 200 & N & N & $<20$ & 150 \\
\hline LH0269C & $N$ & $N$ & 300 & 7 & 500 & N & N & $<20$ & 300 \\
\hline LH0270C & N & $\mathbf{N}$ & 700 & 5 & 200 & N & $<20$ & 20 & 150 \\
\hline LH0279C & N & 20 & 300 & 7 & 1,000 & $N$ & $N$ & $<20$ & 150 \\
\hline LH0272C & $N$ & $N$ & 300 & 5 & 200 & $N$ & N & $<20$ & 70 \\
\hline LH0273C & $N$ & 20 & 200 & 7 & $N$ & $N$ & 20 & 30 & 100 \\
\hline LH0274C & $N$ & 30 & 300 & 2 & N & $N$ & $\mathbf{N}$ & $<20$ & 70 \\
\hline LH0275C & N & $N$ & 7,000 & N & N & N & 20 & $<20$ & 10 \\
\hline LH0276C & $N$ & $N$ & 3,000 & $N$ & 500 & $N$ & 30 & $<20$ & 200 \\
\hline LH0279C & $N$ & 20 & 300 & $N$ & N & N & 20 & 300 & 300 \\
\hline LH028OC & $N$ & 100 & 700 & 2 & 100 & $N$ & N & 30 & 15 \\
\hline LH0281C & $N$ & $N$ & 200 & $N$ & N & $N$ & 30 & N & $<10$ \\
\hline LH0282C & $N$ & $N$ & 1,000 & 5 & 2,000 & $N$ & 50 & $<20$ & 30 \\
\hline LH0283C & N & $N$ & 300 & N & 20 & N & 70 & 20 & 150 \\
\hline LH0284C & N & $\mathbf{N}$ & 200 & 3 & 200 & $N$ & 30 & 150 & 300 \\
\hline LH0285C & $N$ & 50 & 200 & 3 & 1,000 & N & 30 & 30 & 150 \\
\hline LH0286C & N & $\mathbf{N}$ & 300 & 5 & 1,000 & $N$ & 30 & 50 & 100 \\
\hline LH0287C & N & 20 & 150 & 15 & 200 & N & N & $<20$ & 150 \\
\hline LH0288C & $N$ & $\mathbf{N}$ & 500 & 2 & 300 & 50 & 20 & 70 & 150 \\
\hline LH0289C & $N$ & 70 & 200 & 3 & $>2,000$ & $N$ & $N$ & 50 & 300 \\
\hline LH029OC & $N$ & $N$ & 300 & $N$ & $N$ & $N$ & 50 & 30 & 300 \\
\hline LH0291C & $N$ & $N$ & 150 & 3 & 150 & $<50$ & $N$ & N & 100 \\
\hline LH0292C & N & $\mathbf{N}$ & 150 & 5 & 150 & 50 & N & N & 100 \\
\hline LH0293C & $N$ & $N$ & 500 & 3 & 500 & $N$ & $N$ & $N$ & 100 \\
\hline
\end{tabular}


TABLE 2. GEOChEMICAL DATA FOR NONMAGNETIC, hEAVY-MINERAL-CONCENTRATE SAMPLES, LIME hILLS QUADRANGLE, ALASKA--CONTInUEd

Sample Ga ppm-s Ge ppm-s La ppm-s Mn ppm-s Mo ppm-s Nb ppm-s Ni ppm-s Pb ppm-s Pt ppm-s Sb ppm-s

\begin{tabular}{|c|c|c|c|c|c|c|c|c|c|}
\hline LH0238C & 20 & $N$ & 700 & 1,000 & 20 & $<50$ & $<10$ & 300 & N \\
\hline LH0239C & 20 & $N$ & 700 & 700 & 300 & $<50$ & $<10$ & 7,000 & $N$ \\
\hline LH0242C & 15 & $N$ & 2,000 & 700 & $N$ & 50 & 70 & 70 & $N$ \\
\hline LH0243C & $<10$ & $N$ & 1,500 & 150 & $N$ & $<50$ & 150 & 10,000 & N \\
\hline LHO244C & 20 & $N$ & $>2,000$ & 2,000 & $N$ & 70 & 70 & 700 & $N$ \\
\hline LH0245C & 15 & $N$ & 1,500 & 700 & $N$ & 100 & 70 & 1,000 & $N$ \\
\hline LH0246C & 20 & N & 300 & 150 & $N$ & $<50$ & 20 & 70 & $N$ \\
\hline LH0247C & 20 & $N$ & 300 & 700 & $N$ & 70 & 20 & 2,000 & $N$ \\
\hline LH0248C & 10 & $N$ & 700 & 300 & $N$ & $<50$ & 15 & 30 & $N$ \\
\hline LHO25OC & 15 & $\mathbf{N}$ & 300 & 1,000 & $N$ & $<50$ & 150 & 1,000 & $N$ \\
\hline LHO251C & 15 & $N$ & 300 & 1,500 & $N$ & $<50$ & 70 & 70 & N \\
\hline LH0252C & $<10$ & N & 1,000 & 200 & $N$ & $<50$ & $<10$ & 50 & $N$ \\
\hline LH0253C & 15 & $N$ & $>2,000$ & 1,000 & N & 70 & 100 & 70 & N \\
\hline LH0254C & 15 & $N$ & 300 & 700 & $N$ & 70 & 30 & 20 & N \\
\hline LH0255C & 20 & $N$ & 150 & 1,500 & 70 & $<50$ & 150 & 500 & N \\
\hline LH0256C & 15 & $N$ & 200 & 150 & $N$ & 70 & 50 & 50,000 & N \\
\hline LH0257C & $<10$ & $N$ & 700 & 500 & $N$ & $<50$ & $<10$ & 50 & N \\
\hline LHO258C & 30 & $N$ & 200 & 150 & $N$ & $<50$ & 10 & 70 & N \\
\hline LH0259C & 30 & $N$ & 1,500 & 1,000 & 10 & 200 & 10 & 150 & N \\
\hline LH0260C & 15 & $N$ & 300 & 500 & 10 & 100 & 10 & 700 & $N$ \\
\hline LH0261C & 20 & $N$ & 200 & 500 & 500 & $<50$ & $<10$ & 3,000 & $N$ \\
\hline LH0262C & 30 & $N$ & 1,000 & 1,500 & 70 & 70 & $<10$ & 3,000 & $N$ \\
\hline LH0263C & 20 & $N$ & 200 & 700 & 200 & 100 & $<10$ & 1,500 & N \\
\hline LH0264C & 20 & N & 500 & 1,000 & 300 & 50 & $<10$ & 2,000 & N \\
\hline LH0266C & 20 & $N$ & 2,000 & 1,500 & 700 & 70 & $<10$ & 3,000 & N \\
\hline LH0267C & 70 & $N$ & 500 & 3,000 & 70 & 70 & $<10$ & 2,000 & N \\
\hline LH0268C & $N$ & $N$ & $>2,000$ & 300 & 100 & 70 & N & 200 & N \\
\hline LH0269C & $N$ & $N$ & $>2,000$ & 500 & 150 & 100 & $N$ & 300 & N \\
\hline LH027OC & 50 & $N$ & 2,000 & 10,000 & 50 & 70 & $<10$ & 2,000 & $N$ \\
\hline LH0271C & $N$ & $N$ & $>2,000$ & 1,500 & 50 & 70 & $N$ & 300 & $N$ \\
\hline LH0272C & N & $N$ & 1,500 & 700 & 50 & 70 & N & 300 & N \\
\hline LH0273C & $N$ & $N$ & 700 & 300 & 30 & 70 & $<10$ & 300 & N \\
\hline LH0274C & 15 & $N$ & 100 & 500 & 50 & 50 & $<10$ & 500 & N \\
\hline LH0275C & 10 & $N$ & 300 & 300 & 10 & $<50$ & $<10$ & 20 & N \\
\hline LH0276C & 10 & $N$ & 1,500 & 1,500 & 15 & $<50$ & $<10$ & 200 & N \\
\hline LH0279C & 20 & $N$ & 1,500 & 3,000 & 50 & $<50$ & 20 & 700 & N \\
\hline LH0280C & $N$ & $N$ & 2,000 & 700 & 70 & 50 & N & 70 & N \\
\hline LH0281C & N & $N$ & 500 & 150 & $N$ & $N$ & $N$ & 70 & N \\
\hline LH0282C & 20 & $N$ & 150 & 1,500 & 300 & 70 & $<10$ & 10,000 & N \\
\hline LH0283C & $<10$ & $N$ & 700 & 1,000 & $N$ & $<50$ & $<10$ & 200 & N \\
\hline LH0284C & 15 & $N$ & 2,000 & 3,000 & 30 & 100 & 10 & 500 & N \\
\hline LH0285C & 10 & $N$ & $>2,000$ & 2,000 & 700 & 150 & $<10$ & 1,500 & N \\
\hline LH0286C & 15 & $N$ & $>2,000$ & 3,000 & 150 & 100 & $<10$ & 700 & N \\
\hline LH0287C & $<10$ & $N$ & $>2,000$ & 1,000 & 150 & 70 & $N$ & 300 & $N$ \\
\hline LH0288C & 30 & $N$ & 1,500 & 1,500 & 50 & 70 & 20 & 700 & N \\
\hline LH0289C & 10 & $N$ & $>2,000$ & 1,500 & 150 & 50 & $N$ & 700 & N \\
\hline LH0290C & $<10$ & $N$ & 1,500 & 500 & 70 & 100 & $<10$ & 70 & $N$ \\
\hline LH0291C & $<10$ & $N$ & $>2,000$ & 1,000 & 15 & $<50$ & N & 100 & N \\
\hline LH0292C & $<10$ & $N$ & $>2,000$ & 1,000 & 50 & $<50$ & $N$ & 500 & N \\
\hline LH0293C & $<10$ & N & $>2,000$ & 1,000 & 30 & 50 & $N$ & 50 & N \\
\hline
\end{tabular}


TABLE 2. GEOCHEMICAL DATA FOR NONMAGNETIC, hEAVY-MINERAL-CONCENTRATE SAMPLES, LIME hILLS QUADRANGLE, ALASKA--Cont inUed

Sample Sc ppm-s Sn ppm-s Sr ppm-s Th ppm-s $U$ ppm-s $V$ ppm-s $W$ ppm-s $\quad$ Y ppm-s Zn ppm-s Zr ppm-s

\begin{tabular}{|c|c|c|c|c|c|c|c|c|c|c|}
\hline LH0238C & 30 & $>2,000$ & 500 & 500 & N & 100 & 1,000 & 700 & $N$ & $>2,000$ \\
\hline LH0239C & 30 & $>2,000$ & 200 & 700 & $\mathbf{N}$ & 150 & 1,000 & 700 & $\mathbf{N}$ & $>2,000$ \\
\hline LH0242C & 20 & 30 & 1,000 & $<200$ & $N$ & 150 & N & 200 & N & $>2,000$ \\
\hline LH0243C & N & $>2,000$ & 700 & N & N & 70 & 300 & 150 & $\mathbf{N}$ & $>2,000$ \\
\hline LH0244C & 150 & 150 & 500 & 300 & N & 200 & 50 & 300 & $N$ & $>2,000$ \\
\hline LH0245C & 20 & 20 & 700 & N & $N$ & 200 & 70 & 300 & $\mathbf{N}$ & $>2,000$ \\
\hline LH0246C & 30 & 500 & 700 & N & N & 150 & 50 & 150 & N & $>2,000$ \\
\hline LH0247C & 70 & 300 & $<200$ & N & $\mathbf{N}$ & 150 & 50 & 150 & $\mathbf{N}$ & $>2,000$ \\
\hline LH0248C & 15 & 200 & 500 & $N$ & $N$ & 100 & 50 & 700 & $N$ & $>2,000$ \\
\hline LH0250C & 20 & 1,500 & 500 & N & N & 150 & 300 & 100 & 1,500 & $>2,000$ \\
\hline LH0251C & 20 & 500 & 300 & $<200$ & N & 150 & 300 & 150 & N & $>2,000$ \\
\hline LH0252C & 15 & N & 200 & N & N & 100 & $N$ & 700 & N & $>2,000$ \\
\hline LH0253C & 150 & 70 & 700 & 200 & $N$ & 200 & 300 & 300 & 1,000 & $>2,000$ \\
\hline LH0254C & 30 & 150 & 700 & $N$ & $N$ & 200 & 300 & 200 & N & $>2,000$ \\
\hline LH0255C & 20 & 700 & 200 & $N$ & $N$ & 150 & $N$ & 150 & 500 & $>2,000$ \\
\hline LH0256C & 30 & $>2,000$ & 700 & $N$ & N & 150 & 150 & 200 & 700 & $>2,000$ \\
\hline LH0257C & 20 & 150 & 700 & N & $N$ & 70 & 70 & 700 & N & $>2,000$ \\
\hline LH0258C & 10 & $N$ & $<200$ & N & $N$ & 200 & $N$ & 200 & $N$ & $>2,000$ \\
\hline LH0259C & 70 & 100 & 200 & 1,000 & $N$ & 70 & 300 & 700 & $N$ & $>2,000$ \\
\hline LH0260C & 30 & 300 & $<200$ & 300 & N & 70 & 700 & 300 & 700 & $>2,000$ \\
\hline LH0261C & 15 & 300 & 500 & 700 & $N$ & 50 & 150 & 200 & 15,000 & $>2,000$ \\
\hline LH0262C & 70 & 70 & 200 & 300 & N & 70 & 100 & 700 & 20,000 & $>2,000$ \\
\hline LH0263C & 70 & 30 & 200 & $N$ & $N$ & 70 & 150 & 200 & N & $>2,000$ \\
\hline LH0264C & 70 & 700 & 200 & 500 & $\mathbf{N}$ & 70 & 150 & 700 & 7,000 & $>2,000$ \\
\hline LH0266C & 100 & 1,500 & $<200$ & 1,500 & 3,000 & 70 & 150 & 1,500 & N & $>2,000$ \\
\hline LH0267C & 70 & 1,500 & 200 & 1,500 & 2,000 & 70 & 50 & 700 & 1.500 & $>2,000$ \\
\hline LH0268C & 200 & 700 & $<200$ & 3,000 & 5,000 & N & 200 & 1,500 & N & $>2,000$ \\
\hline LH0269C & 200 & 1,000 & $N$ & $>5,000$ & 7,000 & N & 100 & 2,000 & N & $>2,000$ \\
\hline LH0270C & 150 & 500 & $<200$ & 500 & N & 100 & 50 & 1,000 & 2,000 & $>2,000$ \\
\hline LH0271C & 200 & 200 & $<200$ & 5,000 & 5,000 & 70 & 50 & 1,500 & N & $>2,000$ \\
\hline LH0272C & 100 & 700 & N & $>5,000$ & 10,000 & $<20$ & 100 & 1,500 & N & $>2,000$ \\
\hline LH0273C & 100 & 700 & $<200$ & $>5,000$ & 10,000 & 20 & 100 & 2,000 & $\mathbf{N}$ & $>2,000$ \\
\hline LH0274C & 50 & 100 & 200 & 1,000 & 3,000 & 70 & 2,000 & 700 & $N$ & $>2,000$ \\
\hline LH0275C & 15 & N & 300 & N & N & 50 & 2,000 & 200 & N & $>2,000$ \\
\hline LH0276C & 30 & 300 & 500 & 200 & $N$ & 70 & 100 & 500 & 500 & $>2,000$ \\
\hline LH0279C & 100 & $N$ & 500 & 500 & N & 300 & 200 & 700 & N & $>2,000$ \\
\hline LH0280C & 150 & 150 & N & 2,000 & 3,000 & 30 & 500 & 1,000 & $N$ & $>2,000$ \\
\hline LH0281C & 150 & 150 & N & 1,500 & 3,000 & N & 100 & 1,000 & N & $>2,000$ \\
\hline LH0282C & 30 & 700 & $<200$ & N & N & 30 & 15,000 & 500 & 1,500 & $>2,000$ \\
\hline LH0283C & 50 & 150 & 200 & $\mathbf{N}$ & N & 200 & 200 & 700 & N & $>2,000$ \\
\hline LH0284C & 150 & 150 & $<200$ & 500 & N & 200 & 150 & 700 & 500 & $>2,000$ \\
\hline LH0285C & 200 & 200 & $<200$ & 700 & 2,000 & 150 & 10,000 & 1,500 & N & $>2,000$ \\
\hline LH0286C & 200 & 200 & $<200$ & 2,000 & 2,000 & 150 & 300 & 2,000 & 500 & $>2,000$ \\
\hline LH0287C & 200 & 300 & $<200$ & $>5,000$ & 7,000 & 50 & 300 & 5,000 & 1,500 & $>2,000$ \\
\hline LH0288C & 70 & 300 & 500 & 500 & N & 150 & 5,000 & 500 & 2,000 & $>2,000$ \\
\hline LH0289C & 200 & 30 & $<200$ & 3,000 & 3,000 & 70 & 200 & 3,000 & $N$ & $>2,000$ \\
\hline LH0290C & 50 & 70 & $<200$ & 300 & N & 300 & 1,000 & 700 & N & $>2,000$ \\
\hline LH0291C & 200 & 700 & N & 1,500 & 3,000 & 20 & 200 & 3,000 & 2,000 & $>2,000$ \\
\hline LH0292C & 200 & 700 & $\mathbf{N}$ & 1,500 & 3,000 & $<20$ & 200 & 3,000 & 3,000 & $>2,000$ \\
\hline LH0293C & 200 & $>2,000$ & N & 1,500 & 3,000 & 20 & 500 & 2,000 & 1,500 & $>2,000$ \\
\hline
\end{tabular}


TABLE 2. GEOCHEMICAL DATA FOR NONMAGNETIC, hEAVY-MINERAL-CONCENTRATE SAMPLES, LIME hILLS QUADRANGLE, ALASKA--CONTINUEd

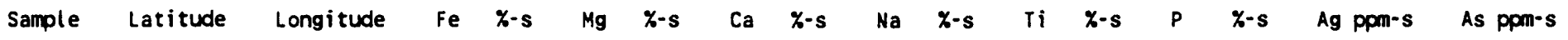

\begin{tabular}{|c|c|c|c|c|c|c|c|c|c|c|c|c|c|}
\hline LH0294C & 615 & 0 & 153 & 19 & 27 & 5.00 & .50 & 7.00 & .7 & 1.000 & 1.0 & $N$ & 2,000 \\
\hline LH0295C & 6156 & 45 & 153 & 59 & 37 & 20.00 & 1.50 & 7.00 & .7 & .300 & $N$ & 300.0 & $>20,000$ \\
\hline LH0296C & 6157 & 55 & 153 & 55 & 24 & 7.00 & 2.00 & 20.00 & .5 & .500 & N & $N$ & N \\
\hline LH0297C & 6156 & 38 & 153 & 54 & 3 & 7.00 & 1.00 & 10.00 & 1.0 & $>2.000$ & 5.0 & 100.0 & 3,000 \\
\hline LH0298C & 6157 & 53 & 154 & 7 & 53 & 5.00 & 2.00 & 15.00 & 1.0 & .500 & $N$ & $N$ & N \\
\hline LH0299C & 6158 & 2 & 154 & 7 & 48 & 2.00 & .05 & 7.00 & $N$ & .300 & $N$ & 3.0 & 2,000 \\
\hline LH0300C & 6142 & 2 & 153 & 46 & 15 & 7.00 & .07 & 15.00 & .5 & $>2.000$ & 7.0 & 10.0 & 10,000 \\
\hline LHo301C & 6141 & 48 & 153 & 44 & 48 & 7.00 & .10 & 2.00 & .7 & 1.500 & 3.0 & 100.0 & $>20,000$ \\
\hline LH0302C & 6139 & 56 & 153 & 41 & 0 & 1.00 & .07 & .30 & $<.5$ & .500 & $N$ & 10.0 & $N$ \\
\hline LH0303C & 6139 & 32 & 153 & 42 & 34 & 1.00 & .07 & 1.00 & .7 & .700 & 1.5 & $N$ & $N$ \\
\hline LH0304C & 6140 & 51 & 153 & 39 & 57 & 2.00 & .07 & 7.00 & .7 & $>2.000$ & .7 & N & N \\
\hline LH0305C & 6141 & 22 & 153 & 36 & 47 & 1.00 & .10 & 1.00 & $<.5$ & 1.500 & $N$ & N & $N$ \\
\hline LH0306C & 6141 & 15 & 153 & 33 & 31 & 3.00 & .15 & 5.00 & .7 & $>2.000$ & 2.0 & $N$ & N \\
\hline LH0307C & 6141 & 11 & 153 & 30 & 32 & 15.00 & 1.00 & 3.00 & .7 & 1.500 & 2.0 & 200.0 & 3,000 \\
\hline LH0308C & 6139 & 1 & 153 & 31 & 33 & 15.00 & .20 & 1.50 & .5 & 1.500 & 1.5 & 30.0 & 10,000 \\
\hline LH0309C & 6138 & 41 & 153 & 31 & 36 & 30.00 & .05 & .50 & $<.5$ & .700 & .7 & 300.0 & $>20,000$ \\
\hline LHO310C & 6138 & 41 & 153 & 31 & 54 & 2.00 & .10 & .50 & $<.5$ & .300 & $N$ & 10.0 & 2,000 \\
\hline LH0311C & 6136 & 33 & 153 & 36 & 8 & 7.00 & .07 & 1.50 & .5 & 1.000 & 1.5 & 300.0 & 10,000 \\
\hline LHO312C & 6137 & 19 & 153 & 30 & 39 & 10.00 & .15 & 1.50 & .7 & $>2.000$ & 2.0 & 100.0 & 3,000 \\
\hline LH0313C & 6132 & 46 & 153 & 38 & 13 & 1.00 & .07 & .70 & $<.5$ & 1.000 & .5 & $N$ & N \\
\hline LHO314C & 6133 & 51 & 153 & 35 & 36 & 5.00 & .30 & 3.00 & .7 & 1.500 & 1.5 & 30.0 & 3,000 \\
\hline LH0315C & 6132 & 53 & 153 & 28 & 22 & 30.00 & .15 & 1.50 & .7 & 1.500 & $N$ & 30.0 & $>20,000$ \\
\hline LH0316C & 6131 & 36 & 153 & 28 & 33 & 30.00 & .30 & .70 & .7 & 1.000 & $N$ & 20.0 & 3,000 \\
\hline LH0317C & 6131 & 3 & 153 & 26 & 50 & 15.00 & .50 & 1.50 & .7 & 1.500 & 1.5 & 2.0 & 500 \\
\hline LH0318C & 6133 & 37 & 153 & 26 & 17 & 15.00 & .30 & 2.00 & .7 & 1.000 & 1.5 & 10.0 & 1,000 \\
\hline LH0319C & 6129 & 47 & 153 & 8 & 54 & 7.00 & .30 & 7.00 & 1.5 & 1.000 & $<.5$ & 15.0 & N \\
\hline LH0320C & 6129 & 50 & 153 & 8 & 42 & 7.00 & .20 & 7.00 & 1.5 & 1.000 & .5 & 15.0 & N \\
\hline LH0321C & 6132 & 37 & 153 & 9 & 14 & 30.00 & $<.05$ & 3.00 & $N$ & .150 & 3.0 & 70.0 & N \\
\hline LH0322C & 6133 & 8 & 153 & 10 & 23 & 7.00 & .10 & 15.00 & .5 & .700 & 20.0 & 150.0 & 3,000 \\
\hline LH0323C & 6133 & 2 & 153 & 10 & 32 & 7.00 & $<.05$ & 2.00 & 1.0 & .500 & 1.5 & 150.0 & N \\
\hline LH0324C & 6133 & 22 & 153 & 5 & 8 & 2.00 & $<.05$ & 20.00 & .5 & 1.500 & 1.0 & 20.0 & 7,000 \\
\hline LH0325C & 6136 & 12 & 153 & 7 & 13 & 2.00 & .20 & 5.00 & .7 & .700 & 5.0 & 50.0 & 700 \\
\hline LH0326C & 6137 & 15 & 153 & 6 & 51 & 5.00 & 1.50 & 5.00 & 1.0 & .700 & 1.5 & 2.0 & 500 \\
\hline LH0327C & 6138 & 41 & 153 & 6 & 12 & 5.00 & .10 & 10.00 & .7 & 1.500 & 3.0 & 100.0 & 1,000 \\
\hline LH0328C & 6139 & 22 & 153 & 10 & 10 & .30 & $<.05$ & 20.00 & $<.5$ & .150 & .5 & 5.0 & $<500$ \\
\hline LH0329C & 6138 & 7 & 153 & 11 & 35 & 7.00 & .07 & 5.00 & .5 & .500 & 3.0 & 300.0 & $>20,000$ \\
\hline LH0330C & 6141 & 20 & 153 & 1 & 56 & 1.00 & .10 & 10.00 & .7 & .700 & 7.0 & 50.0 & 7,000 \\
\hline LH0332C & 6142 & 4 & 153 & 9 & 6 & 7.00 & 1.00 & 10.00 & .7 & 1.000 & 3.0 & 10.0 & 7,000 \\
\hline LhO333C & 6141 & 25 & 153 & 2 & 0 & 2.00 & .30 & 15.00 & .7 & 1.000 & 3.0 & 20.0 & 10,000 \\
\hline LH0334C & 6159 & 58 & 153 & 53 & 44 & 2.00 & 3.00 & 15.00 & .5 & .200 & $N$ & 1.0 & 5,000 \\
\hline LH0335C & 6145 & 56 & 153 & 38 & 44 & 2.00 & .30 & 5.00 & .7 & 1.500 & 3.0 & 15.0 & 1,500 \\
\hline LH0336C & 6147 & 29 & 153 & 41 & 5 & 7.00 & .20 & 5.00 & .7 & 1.000 & 2.0 & 100.0 & 10,000 \\
\hline LH0337C & 6148 & 8 & 153 & 48 & 23 & 3.00 & $<.05$ & 2.00 & $<.5$ & .200 & .7 & 5.0 & $>20,000$ \\
\hline LH0338C & 6147 & 41 & 153 & 50 & 8 & 2.00 & $<.05$ & 3.00 & .7 & .500 & .5 & 2.0 & 7,000 \\
\hline LH0339C & 6146 & 55 & 153 & 48 & 21 & .70 & $<.05$ & 1.00 & $<.5$ & .700 & 1.0 & 20.0 & 7,000 \\
\hline LH0340C & 6146 & 33 & 153 & 48 & 16 & .30 & $<.05$ & 1.50 & .5 & $>2.000$ & .7 & 20.0 & 700 \\
\hline LH0341C & 6147 & 10 & 153 & 32 & 54 & 1.00 & 1.00 & 5.00 & .7 & $>2.000$ & 5.0 & $N$ & N \\
\hline LH0342C & 6148 & 9 & 153 & 33 & 32 & 3.00 & .15 & 5.00 & .5 & 1.000 & 3.0 & 70.0 & 10,000 \\
\hline LH0343C & 6149 & 14 & 153 & 273 & 30 & 7.00 & .10 & 7.00 & .5 & 2.000 & 7.0 & 15.0 & $>20,000$ \\
\hline LH0344C & 6151 & 7 & 153 & 263 & 37 & 7.00 & .05 & 10.00 & 1.0 & .100 & 7.0 & $N$ & 3,000 \\
\hline
\end{tabular}


TABLE 2. GEOCHEMICAL DATA FOR NONMAGNETIC, hEAVY-MINERAL-CONCENTRATE SAMPLES, LIME HILLS QUADRANGLE, ALASKA--CONT INUEd

Sample AU ppm-s B ppm-s Ba ppm-s Be ppm-s Bi ppm-s l Cd ppm-s Co ppm-s Cr ppm-s Cu ppm-s

\begin{tabular}{|c|c|c|c|c|c|c|c|c|c|}
\hline LH0294C & $N$ & 30 & 700 & 3 & $<20$ & $N$ & 30 & 30 & 150 \\
\hline LH0295C & N & 500 & 3,000 & 2 & 50 & N & 70 & 30 & 200 \\
\hline LH0296C & $N$ & 300 & 2,000 & 5 & N & N & 20 & 70 & 100 \\
\hline LH0297C & $N$ & 100 & 7,000 & 5 & $>2,000$ & N & 100 & 70 & 150 \\
\hline LH0298C & $N$ & 500 & 1,500 & 5 & N & N & $<20$ & 50 & 100 \\
\hline LH0299C & N & 50 & 3,000 & 10 & N & $N$ & $<20$ & $<20$ & 50 \\
\hline LH030OC & $N$ & N & 2,000 & 2 & 200 & $N$ & 700 & $<20$ & 200 \\
\hline LH0301C & N & 200 & 500 & 5 & 500 & $N$ & 700 & $<20$ & 1,500 \\
\hline LH0302C & $N$ & 50 & 3,000 & 3 & N & $N$ & 20 & 100 & 20 \\
\hline LH0303C & $N$ & 50 & 700 & N & $\mathbf{N}$ & $N$ & 20 & 70 & 20 \\
\hline LH0304C & N & 20 & 3,000 & N & $N$ & N & 30 & 50 & 150 \\
\hline LH0305C & $N$ & 70 & 7,000 & N & N & $N$ & N & 150 & 15 \\
\hline LH0306C & $N$ & 100 & 5,000 & N & 20 & $N$ & 20 & 70 & 100 \\
\hline LH0307C & $N$ & 100 & $>10,000$ & 3 & 200 & N & 100 & 700 & 500 \\
\hline LH0308C & $N$ & 50 & $>10,000$ & 2 & 30 & $N$ & 50 & 70 & 200 \\
\hline LH0309C & 50 & $N$ & $>10,000$ & N & 700 & $N$ & 150 & $<20$ & 200 \\
\hline LH0310C & $N$ & 50 & 1,500 & 3 & 30 & N & 50 & 100 & 70 \\
\hline LH0311C & $N$ & 200 & $>10,000$ & 7 & 50 & $N$ & 30 & 50 & 200 \\
\hline LH0312C & $N$ & 70 & $>10,000$ & $N$ & 30 & N & 70 & 50 & 150 \\
\hline LH0313C & N & 30 & 1,000 & 7 & $N$ & N & $<20$ & 50 & 20 \\
\hline LH0314C & $<20$ & 70 & 2,000 & 5 & 70 & $N$ & 30 & 200 & 1,000 \\
\hline LH0315C & N & 700 & 700 & N & $>2,000$ & $N$ & 1,000 & 30 & 1,000 \\
\hline LH0316C & $N$ & 2,000 & 10,000 & $N$ & 500 & $N$ & 1,500 & 50 & 500 \\
\hline LH0317C & N & 70 & 5,000 & 5 & 20 & N & 100 & 300 & 500 \\
\hline LH0318C & N & 50 & $>10,000$ & 3 & 50 & N & 100 & 200 & 500 \\
\hline LH0319C & $N$ & N & 500 & 5 & 30 & N & $<20$ & $<20$ & 500 \\
\hline LH0320C & $N$ & $N$ & 700 & 5 & N & $N$ & $<20$ & $<20$ & 70 \\
\hline LH0321C & N & $N$ & $>10,000$ & $N$ & 20 & 200 & 200 & $<20$ & 700 \\
\hline LH0322C & $N$ & $N$ & $>10,000$ & N & 1,500 & 70 & 30 & $<20$ & 1,000 \\
\hline LH0323C & 100 & $N$ & 7,000 & 5 & 700 & $<50$ & 30 & $<20$ & 100 \\
\hline LH0324C & N & $N$ & 700 & 3 & 200 & $\mathbf{N}$ & 30 & $<20$ & 10 \\
\hline LH0325C & $\mathbf{N}$ & $N$ & 700 & 2 & $>2,000$ & N & $<20$ & 30 & 700 \\
\hline LH0326C & N & N & 300 & 5 & 500 & N & $<20$ & 50 & 500 \\
\hline LH0327C & $\mathbf{N}$ & 150 & 1,000 & 3 & 1,500 & N & 20 & $<20$ & 300 \\
\hline LH0328C & N & $N$ & 70 & 15 & 700 & N & $N$ & N & 15 \\
\hline LH0329C & 20 & $N$ & 700 & 2 & $>2,000$ & $N$ & 300 & $<20$ & 700 \\
\hline LH0330C & $N$ & N & 150 & N & 300 & N & 70 & $<20$ & 300 \\
\hline LH0332C & $N$ & 500 & 700 & 5 & 2,000 & N & 50 & 70 & 700 \\
\hline Lн0333C & $N$ & N & 300 & 70 & 500 & $N$ & 70 & 30 & 500 \\
\hline LH0334C & $\mathbf{N}$ & 700 & $>10,000$ & 2 & $<20$ & $N$ & 30 & 30 & 50 \\
\hline LH0335C & 30 & 700 & $>10,000$ & 3 & 500 & N & 100 & 300 & 700 \\
\hline LH0336C & 70 & 50 & $>10,000$ & $N$ & $<20$ & $N$ & 150 & 70 & 150 \\
\hline LH0337C & N & $N$ & 300 & 3 & 200 & $N$ & 700 & $<20$ & 30 \\
\hline LH0338C & $N$ & N & 150 & 3 & 1,000 & N & $N$ & N & 100 \\
\hline LH0339C & N & N & 70 & 5 & 1,500 & $N$ & $N$ & N & 20 \\
\hline LH0340C & $\mathbf{N}$ & 100 & 7,000 & 50 & 300 & $N$ & $N$ & $<20$ & $<10$ \\
\hline LH0341C & N & 20 & 7,000 & 2 & $N$ & N & $<20$ & 200 & 10 \\
\hline LH0342C & N & 100 & $>10,000$ & $N$ & 20 & N & 70 & 30 & 150 \\
\hline LH0343C & N & 50 & $>10,000$ & $N$ & 150 & N & 150 & 50 & 100 \\
\hline LH0344C & N & $N$ & 700 & N & $<20$ & $N$ & 20 & $<20$ & 30 \\
\hline
\end{tabular}


TABLE 2. GEOCHEMICAL DATA FOR NONMAGNETIC, hEAVY-MINERAL-CONCENTRATE SAMPLES, LIME HILLS QUADRANGLE, ALASKA--CONT InUEd

Sample Ga ppm-s Ge ppm-s La ppm-s Mn ppm-s Mo ppm-s Nb ppm-s Ni ppm-s Pb ppm-s Pt ppm-s Sb ppm-s

\begin{tabular}{|c|c|c|c|c|c|c|c|c|c|c|}
\hline LH0294C & 20 & $N$ & 300 & 1,000 & 10 & $<50$ & 20 & 70 & $N$ & N \\
\hline LH0295C & 20 & $\mathbf{N}$ & 100 & 500 & 20 & $<50$ & 50 & 50,000 & $N$ & 2,000 \\
\hline LH0296C & 20 & $N$ & 100 & 700 & $N$ & $<50$ & 50 & 100 & $N$ & N \\
\hline LH0297C & 20 & $\mathbf{N}$ & 300 & 500 & 200 & 100 & 50 & 15,000 & N & $<200$ \\
\hline LH0298C & 20 & $N$ & 300 & 500 & $N$ & $<50$ & 20 & 30 & N & N \\
\hline LH0299C & N & $N$ & 2,000 & 150 & 30 & 50 & $<10$ & 700 & $N$ & $N$ \\
\hline LH0300C & $<10$ & $N$ & 700 & 500 & $N$ & 100 & 100 & 500 & N & $N$ \\
\hline LH0301C & 15 & $N$ & 1,500 & 500 & $N$ & 70 & 200 & 3,000 & $N$ & $N$ \\
\hline LHO302C & 30 & $N$ & N & 70 & $N$ & $<50$ & $<10$ & 300 & N & N \\
\hline LH0303C & 30 & $N$ & N & 150 & $\mathbf{N}$ & $<50$ & 10 & N & N & N \\
\hline LH0304C & 20 & N & 1,000 & 500 & 10 & 150 & $<10$ & 30 & $N$ & $N$ \\
\hline LH0305C & 30 & N & 100 & 150 & N & 70 & $<10$ & $N$ & N & $N$ \\
\hline LHO306C & 20 & $N$ & 700 & 500 & N & 150 & 30 & 300 & N & 300 \\
\hline LH0307C & 10 & $N$ & 150 & 1,500 & $N$ & 50 & 150 & 15,000 & $N$ & $<200$ \\
\hline LH0308C & $<10$ & $\mathbf{N}$ & 150 & 500 & $N$ & 70 & 70 & 5,000 & $N$ & N \\
\hline LHO309C & 10 & $N$ & 100 & 70 & $N$ & $<50$ & 100 & 7,000 & $N$ & 3,000 \\
\hline LH031OC & 30 & $N$ & N & 100 & N & $<50$ & 20 & 500 & $N$ & N \\
\hline LH0311C & 20 & $N$ & $N$ & 150 & $N$ & $<50$ & 30 & 50,000 & $N$ & 700 \\
\hline LH0312C & $<10$ & $N$ & 100 & 150 & $N$ & 100 & 70 & 3,000 & $N$ & $N$ \\
\hline LH0313C & 30 & $N$ & $N$ & 100 & 15 & $<50$ & $<10$ & 70 & $N$ & $N$ \\
\hline LH0314C & 30 & $N$ & 150 & 700 & 150 & $<50$ & 20 & 3,000 & $N$ & $N$ \\
\hline LH0315C & 10 & $N$ & N & 70 & 70 & 70 & 300 & 150 & $N$ & $<200$ \\
\hline LH0316C & 15 & $N$ & N & 70 & N & 50 & 700 & 1,500 & N & $N$ \\
\hline LHO317C & 20 & $N$ & 100 & 3,000 & $N$ & $<50$ & 200 & 1,000 & N & $<200$ \\
\hline LHO318C & 15 & $N$ & N & 1,000 & $N$ & $<50$ & 150 & 1,500 & N & $<200$ \\
\hline LHO3t9C & 30 & $N$ & 300 & 1,500 & 50 & $<50$ & $<10$ & 200 & $N$ & N \\
\hline LH032OC & 30 & $N$ & 150 & 1,000 & 70 & 50 & $<10$ & 200 & $N$ & $N$ \\
\hline LH0321C & 10 & N & 200 & 300 & 150 & $<50$ & 70 & 200 & $N$ & $N$ \\
\hline LH0322C & $<10$ & $N$ & 1,500 & 1,500 & 300 & $<50$ & $<10$ & 15,000 & $N$ & N \\
\hline LH0323C & $<10$ & $N$ & 200 & 300 & 30 & $<50$ & $<10$ & 300 & $N$ & $N$ \\
\hline LH0324C & $<10$ & N & 1,500 & 150 & 15 & 150 & $<10$ & 500 & $N$ & $N$ \\
\hline LH0325C & 10 & N & 300 & 700 & 300 & $<50$ & $<10$ & 2,000 & $N$ & $N$ \\
\hline LH0326C & 50 & N & 1,500 & 2,000 & 500 & $<50$ & $<10$ & 1,000 & $N$ & $N$ \\
\hline LH0327C & 15 & $N$ & 300 & 700 & 1,500 & $<50$ & $<10$ & 15,000 & $N$ & $N$ \\
\hline LH0328C & $<10$ & $N$ & 300 & 150 & 200 & 70 & N & 700 & $N$ & $N$ \\
\hline LH0329C & $N$ & $N$ & 300 & 200 & 500 & $<50$ & 50 & 2,000 & $N$ & $N$ \\
\hline LH0330C & $<10$ & $N$ & 500 & 700 & 1,500 & 50 & $<10$ & $>50,000$ & $N$ & N \\
\hline LH0332C & 20 & $N$ & 300 & 1,500 & 20 & 50 & 50 & 1,500 & $N$ & $N$ \\
\hline LH0333C & $<10$ & $N$ & 1,500 & 700 & 500 & 70 & $<10$ & 7,000 & $N$ & $N$ \\
\hline LH0334C & 15 & $N$ & N & 300 & 50 & $<50$ & 50 & 500 & $\mathbf{N}$ & $N$ \\
\hline LH0335C & 30 & $N$ & 300 & 500 & 20 & 50 & 70 & 700 & N & N \\
\hline LH0336C & 15 & $N$ & $<100$ & 150 & N & $<50$ & 100 & 1,500 & $N$ & 300 \\
\hline LH0337C & $N$ & $N$ & $N$ & 70 & 150 & $<50$ & 70 & 700 & $N$ & $N$ \\
\hline LH0338C & $N$ & $N$ & N & 100 & 1,500 & 70 & $N$ & 70 & $N$ & $N$ \\
\hline LH0339C & $N$ & N & 150 & 150 & 1,000 & 50 & $N$ & 3,000 & $N$ & N \\
\hline LH034OC & N & N & $N$ & 100 & 500 & 100 & $N$ & 500 & N & N \\
\hline LH0341C & 10 & $N$ & 700 & 200 & $N$ & 70 & 20 & 150 & $N$ & $N$ \\
\hline LH0342C & 10 & $N$ & 200 & 150 & $N$ & $<50$ & 70 & 1,500 & $N$ & $<200$ \\
\hline LH0343C & 10 & N & 300 & 150 & $N$ & $<50$ & 30 & 700 & $N$ & $<200$ \\
\hline LH0344C & $<10$ & $N$ & 300 & 100 & $N$ & N & 10 & $<20$ & $N$ & $N$ \\
\hline
\end{tabular}




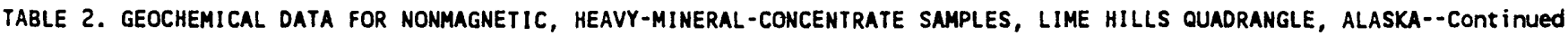

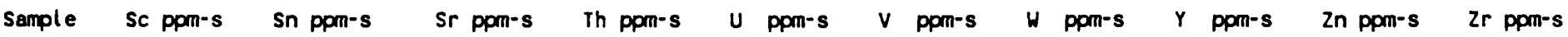

\begin{tabular}{|c|c|c|c|c|c|c|c|c|c|c|}
\hline LH0294C & 10 & 50 & 500 & $\mathbf{N}$ & $N$ & 150 & 500 & 500 & N & $>2,000$ \\
\hline LH0295C & $<10$ & 700 & 300 & $N$ & $N$ & 150 & 150 & 70 & 1,500 & $>2,000$ \\
\hline LH0296C & 15 & $N$ & 500 & $\mathbf{N}$ & $N$ & 300 & N & 70 & N & $>2,000$ \\
\hline LH0297C & 20 & 300 & 700 & N & $N$ & 300 & 2,000 & 500 & $N$ & $>2,000$ \\
\hline LH0298C & 10 & $\mathbf{N}$ & 500 & 200 & N & 200 & N & 300 & N & $>2,000$ \\
\hline LH0299C & 10 & 50 & $<200$ & $>5,000$ & 5,000 & $N$ & N & 2,000 & N & $>2,000$ \\
\hline LH0300C & 100 & 700 & 700 & 200 & N & 700 & 700 & 500 & $N$ & $>2,000$ \\
\hline LH0301C & 100 & $>2,000$ & $<200$ & 200 & N & 20 & 10,000 & 500 & 500 & $>2,000$ \\
\hline LHO3O2C & $N$ & N & $<200$ & N & $N$ & 300 & 500 & 50 & N & $>2,000$ \\
\hline LH0303C & $N$ & $N$ & $<200$ & N & $\mathbf{N}$ & 150 & 50 & 100 & $N$ & $>2,000$ \\
\hline LHO3OLC & $<10$ & 50 & 500 & $200^{\circ}$ & $N$ & 700 & N & 500 & N & $>2,000$ \\
\hline LH0305C & $N$ & N & $<200$ & $N$ & $N$ & 300 & 300 & 150 & N & $>2,000$ \\
\hline LH0306C & 10 & 20 & 500 & $N$ & N & 300 & 100 & 300 & 1,000 & $>2,000$ \\
\hline LH0307C & 30 & $>2,000$ & 700 & $N$ & N & 200 & 5,000 & 100 & 1,500 & $>2,000$ \\
\hline LH0308C & 15 & 700 & 700 & N & N & 100 & N & 100 & N & $>2,000$ \\
\hline LH0309C & $<10$ & $>2,000$ & 300 & $N$ & N & 70 & N & 70 & 700 & $>2,000$ \\
\hline LH0310C & $N$ & 1,000 & $<200$ & $N$ & $N$ & 150 & 300 & 70 & N & $>2,000$ \\
\hline LH0311C & 10 & 2,000 & 700 & N & $\mathbf{N}$ & 150 & 700 & 100 & N & $>2,000$ \\
\hline LHO312C & 20 & 100 & 700 & N & N & 150 & 50 & 100 & 1,000 & $>2,000$ \\
\hline LHO313C & 10 & N & $<200$ & N & N & 300 & 50 & 100 & N & $>2,000$ \\
\hline LH0314C & 20 & 100 & 200 & N & N & 300 & 150 & 100 & N & $>2,000$ \\
\hline LH0315C & 15 & $>2,000$ & 200 & $N$ & N & 100 & 15,000 & 150 & $N$ & $>2,000$ \\
\hline LH0316C & 10 & 300 & 200 & $N$ & N & 150 & 3,000 & 50 & N & 300 \\
\hline LH0317C & 20 & N & 500 & $N$ & N & 200 & N & 70 & 700 & $>2,000$ \\
\hline LH0318C & 15 & 20 & 300 & N & N & 150 & 2,000 & 70 & 500 & $>2,000$ \\
\hline LH0319C & 50 & 50 & 500 & 200 & N & 200 & 100 & 200 & N & $>2,000$ \\
\hline LH0320C & 50 & $N$ & 700 & N & $N$ & 300 & 300 & 100 & N & $>2,000$ \\
\hline LH0321C & N & N & 700 & N & N & $<20$ & $N$ & 70 & $>20,000$ & $>2,000$ \\
\hline LH0322C & 20 & 1,000 & 700 & $<200$ & N & 50 & 150 & 700 & 10,000 & $>2,000$ \\
\hline LH0323C & 70 & 500 & $<200$ & 1,500 & 2,000 & $<20$ & 150 & 1,500 & 2,000 & $>2,000$ \\
\hline LH0324C & 70 & $>2,000$ & $<200$ & 200 & N & $<20$ & 1,000 & 1,500 & N & $>2,000$ \\
\hline LH0325C & 30 & 2,000 & $<200$ & 200 & N & 50 & 300 & 700 & 1,000 & $>2,000$ \\
\hline LH0326C & 70 & 1,500 & $<200$ & 700 & 1,000 & 70 & 150 & 700 & N & $>2,000$ \\
\hline LH0327C & 20 & $>2,000$ & 200 & 700 & N & 100 & 300 & 700 & 1,500 & $>2,000$ \\
\hline LH0328C & 20 & 1,000 & $<200$ & 1,500 & 3,000 & $N$ & 200 & $>5,000$ & N & $>2,000$ \\
\hline LH0329C & 20 & 1,500 & $<200$ & 500 & N & 50 & 1,000 & 700 & 700 & $>2,000$ \\
\hline LH0330C & 30 & $>2,000$ & 200 & 700 & $N$ & 300 & 5,000 & 300 & N & $>2,000$ \\
\hline LH0332C & 50 & $>2,000$ & 200 & 500 & N & 150 & N & 1,000 & N & $>2,000$ \\
\hline LH0333C & 50 & $>2,000$ & $<200$ & 3,000 & 2,000 & 70 & 1,500 & 500 & N & $>2,000$ \\
\hline LH0334C & 10 & 50 & 700 & N & N & 100 & 300 & 100 & $N$ & $>2,000$ \\
\hline LH0335C & 30 & 700 & 300 & N & N & 300 & 2,000 & 150 & $N$ & $>2,000$ \\
\hline LH0336C & $<10$ & 70 & 700 & N & N & 100 & 5,000 & 100 & $N$ & $>2,000$ \\
\hline LH0337C & 50 & 500 & N & $>5,000$ & 5,000 & $<20$ & 1,000 & 1,000 & N & $>2,000$ \\
\hline LH0338C & 20 & 150 & $N$ & 1,500 & 2,000 & $N$ & 15,000 & 1,000 & $N$ & $>2,000$ \\
\hline LH0339C & 50 & 1,500 & N & 3,000 & 5,000 & N & 2,000 & 1,500 & $\mathbf{N}$ & $>2,000$ \\
\hline LH0340C & 50 & 300 & $\mathbf{N}$ & 1,500 & 2,000 & $<20$ & 15,000 & 700 & N & $>2,000$ \\
\hline LH0341C & 20 & 200 & 500 & N & N & 150 & 50 & 200 & $N$ & $>2,000$ \\
\hline LH0342C & 10 & $>2,000$ & 1,500 & N & N & 70 & 700 & 150 & N & $>2,000$ \\
\hline LH0343C & 10 & 500 & 1,000 & N & $N$ & 70 & N & 300 & $N$ & $>2,000$ \\
\hline LH0344C & 15 & 70 & $<200$ & N & N & $<20$ & $N$ & 700 & $N$ & $>2,000$ \\
\hline
\end{tabular}




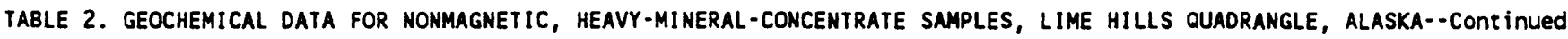

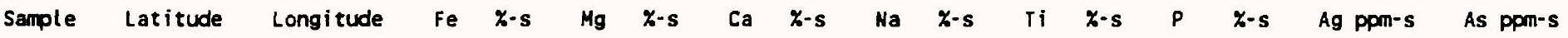

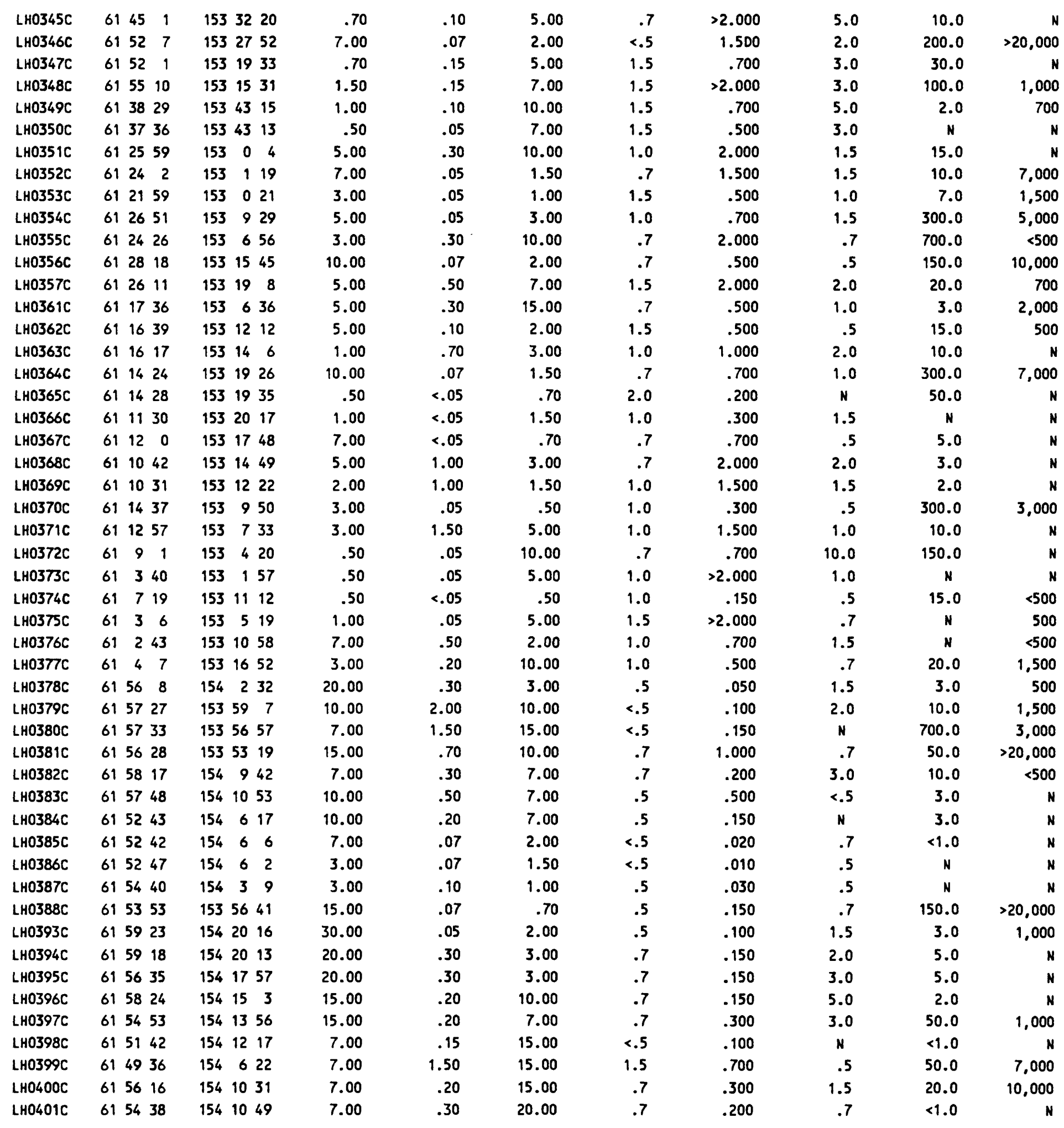


TABLE 2. GEOCHEMICAL DATA FOR NONMAGNETIC, heAVY-MINERAL-CONCENTRATE SAMPLES, LIME hILLS QUADRANGLE, ALASKA--ContinUEd

Sample AU ppm-s B ppm-s Ba ppm-s $\quad$ Be ppm-s $\quad$ Bi ppm-s $\quad$ Cd ppm-s Co ppm-s Cr ppm-s CU ppm-s

\begin{tabular}{|c|c|c|c|c|c|c|c|c|c|}
\hline LH0345C & $N$ & N & $>10,000$ & 2 & N & 100 & $<20$ & 300 & 20 \\
\hline LH0346C & 300 & $N$ & $>10,000$ & $N$ & 700 & N & 300 & 30 & 50 \\
\hline LH0347C & 30 & 70 & 700 & $\mathbf{N}$ & N & N & N & 50 & 15 \\
\hline LH0348C & 20 & 100 & 2,000 & 2 & 700 & N & 30 & 30 & 20 \\
\hline LH0349C & $N$ & N & 300 & $N$ & 30 & N & 30 & $<20$ & 30 \\
\hline LHO350C & $N$ & $N$ & 2,000 & $N$ & $N$ & N & $<20$ & $<20$ & 100 \\
\hline LH0351C & $\mathbf{N}$ & N & 10,000 & 5 & 500 & N & 20 & 30 & 500 \\
\hline LH0352C & N & N & 5,000 & 5 & N & N & 300 & $<20$ & 100 \\
\hline LHO353C & $\mathbf{N}$ & N & 700 & 3 & 150 & N & 50 & $<20$ & 150 \\
\hline LH0354C & 70 & $N$ & 500 & 3 & 1,500 & $N$ & 30 & $<20$ & 100 \\
\hline LH0355C & $\mathbf{N}$ & N & $>10,000$ & 3 & 50 & 700 & $<20$ & 30 & 500 \\
\hline LH0356C & $N$ & N & 300 & 3 & $>2,000$ & N & 150 & $<20$ & 10,000 \\
\hline LH0357C & $N$ & N & 500 & 5 & 1,000 & N & $<20$ & $<20$ & 300 \\
\hline LH0361C & $N$ & N & 300 & 3 & N & $N$ & 70 & 30 & 50 \\
\hline LH0362C & N & N & 700 & 3 & 150 & $N$ & $<20$ & $<20$ & 300 \\
\hline LH0363C & N & N & 300 & 2 & 2,000 & $N$ & $<20$ & 150 & 15 \\
\hline LH0364C & N & N & 150 & 3 & $>2,000$ & $N$ & 30 & $<20$ & 300 \\
\hline LH0365C & N & N & 500 & N & $>2,000$ & $N$ & $<20$ & $<20$ & 15 \\
\hline LH0366C & $N$ & $\mathbf{N}$ & 200 & $N$ & 700 & $N$ & $<20$ & $<20$ & 15 \\
\hline LH0367C & $N$ & 20 & 300 & 2 & 700 & N & 100 & $<20$ & 300 \\
\hline LH0368C & N & $\mathbf{N}$ & 1,000 & 2 & 100 & $N$ & 50 & 150 & 30 \\
\hline LH0369C & $N$ & N & 200 & 20 & 500 & N & $N$ & 150 & 30 \\
\hline LH0370C & $\mathbf{N}$ & N & 200 & $N$ & 1,500 & N & N & $<20$ & 100 \\
\hline LH0371C & $N$ & N & 300 & 3 & 1,000 & 200 & 30 & 100 & 150 \\
\hline LH0372C & $N$ & N & 300 & $N$ & $>2,000$ & N & N & $<20$ & 15 \\
\hline LH0373C & $N$ & N & 200 & N & N & N & $<20$ & $<20$ & 150 \\
\hline LH0374C & N & N & 500 & N & 1,000 & 150 & N & $<20$ & 300 \\
\hline LH0375C & N & 20 & 1,500 & N & N & N & 30 & $<20$ & 30 \\
\hline LH0376C & N & N & 5,000 & $N$ & N & N & 500 & $<20$ & 300 \\
\hline LH0377C & N & N & $>10,000$ & 10 & N & 150 & 20 & $<20$ & 20 \\
\hline LH0378C & N & 70 & $>10,000$ & $\mathbf{N}$ & $N$ & N & 100 & $<20$ & 150 \\
\hline LH0379C & N & 300 & $>10,000$ & $N$ & 20 & N & 100 & 20 & 70 \\
\hline LH0380C & N & 200 & $>10,000$ & 3 & 500 & N & 30 & $<20$ & 200 \\
\hline LH0381C & N & 500 & 700 & 7 & 300 & $\mathbf{N}$ & 1,000 & 30 & 1,000 \\
\hline LH0382C & N & 30 & $>10,000$ & N & 200 & N & 20 & $<20$ & 150 \\
\hline LH0383C & N & N & $>10,000$ & 15 & N & N & 50 & $<20$ & 150 \\
\hline LH0384C & N & N & $>10,000$ & $N$ & $N$ & $N$ & 30 & $<20$ & 150 \\
\hline LH0385C & $N$ & N & $>10,000$ & N & $N$ & N & $<20$ & $<20$ & 100 \\
\hline LH0386C & N & N & $>10,000$ & N & N & N & $<20$ & $<20$ & 30 \\
\hline LH0387C & N & $N$ & $>10,000$ & N & N & N & $<20$ & $<20$ & 50 \\
\hline LH0388C & 30 & 500 & 1,500 & N & 700 & N & 2,000 & $<20$ & 300 \\
\hline LH0393C & N & N & $>10,000$ & $N$ & N & N & 300 & $<20$ & 500 \\
\hline LH0394C & N & 70 & 10,000 & N & N & N & 200 & 30 & 700 \\
\hline LH0395C & $N$ & 50 & $>10,000$ & N & N & N & 100 & 20 & 2,000 \\
\hline LHO396C & N & 100 & $>10,000$ & N & $N$ & N & 70 & 20 & 150 \\
\hline LH0397C & N & 70 & $>10,000$ & $N$ & 70 & $N$ & 100 & 70 & 200 \\
\hline LH0398C & $N$ & $N$ & $>10,000$ & N & $N$ & N & 70 & $<20$ & 100 \\
\hline LH0399C & $\mathbf{N}$ & 100 & $>10,000$ & N & $<20$ & $N$ & 300 & 20 & 30 \\
\hline LHO4OOC & $N$ & 50 & $>10,000$ & $N$ & 1,000 & $N$ & 30 & 30 & 200 \\
\hline LH0401C & N & $N$ & $>10,000$ & $N$ & N & $N$ & 30 & $<20$ & 100 \\
\hline
\end{tabular}




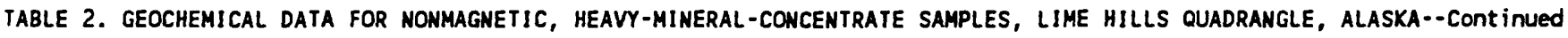

Sample Ga ppm-s Ge ppm-s La ppm-s Mn ppm-s Mo ppm-s Nb ppm-s Ni ppm-s Pb ppm-s Pt ppm-s Sb ppm-s

\begin{tabular}{|c|c|c|c|c|c|c|c|c|c|c|}
\hline LH0345C & 10 & $N$ & 100 & 150 & N & 70 & 10 & 5,000 & N & 500 \\
\hline LH0346C & 10 & $\mathbf{N}$ & 200 & 150 & 15 & $<50$ & 150 & 2,000 & N & $<200$ \\
\hline LH0347C & 15 & $N$ & 200 & 500 & $N$ & $<50$ & $<10$ & 30 & N & N \\
\hline LH0348C & 20 & $\mathbf{N}$ & 150 & 700 & 50 & 50 & 10 & 5,000 & N & N \\
\hline LH0349C & 10 & $N$ & 300 & 300 & N & $<50$ & $<10$ & 70 & N & N \\
\hline LH0350C & 10 & $N$ & 300 & 150 & $N$ & $<50$ & $<10$ & $<20$ & $N$ & N \\
\hline LH0351C & 20 & $N$ & 1,500 & 700 & 30 & 100 & $<10$ & 7,000 & $N$ & N \\
\hline LH0352C & $\mathbf{N}$ & N & 300 & 150 & 200 & 70 & 20 & 1,000 & $\mathbf{N}$ & N \\
\hline LH0353C & 10 & $\mathbf{N}$ & N & 150 & 70 & $<50$ & $<10$ & 500 & $N$ & N \\
\hline LH0354C & 10 & N & 300 & 300 & 200 & $<50$ & N & 7,000 & $N$ & N \\
\hline LH0355C & 20 & $N$ & 1,500 & 1,000 & 150 & 70 & $\mathbf{N}$ & 15,000 & N & N \\
\hline LH0356C & 15 & $N$ & 1,500 & 100 & 1,000 & 50 & $N$ & 7,000 & $N$ & N \\
\hline LH0357C & 30 & N & 700 & 2,000 & N & 70 & $\mathbf{N}$ & 1,000 & $N$ & N \\
\hline LH0361C & 30 & N & N & 700 & 70 & 50 & 30 & 200 & $N$ & $N$ \\
\hline LH0362C & 20 & $N$ & 1,000 & 1,000 & 150 & $<50$ & N & 1,500 & N & $N$ \\
\hline LH0363C & $<10$ & $N$ & 2,000 & 700 & 150 & 50 & N & 1,000 & $N$ & N \\
\hline LH0364C & N & $N$ & 1,500 & 500 & 200 & $<50$ & N & 500 & N & N \\
\hline LH0365C & $<10$ & $N$ & N & 70 & 200 & $<50$ & N & 200 & N & N \\
\hline LH0366C & $<10$ & N & N & 100 & 200 & $<50$ & N & 300 & N & N \\
\hline LH0367C & $<10$ & N & 1,000 & 150 & 300 & 70 & $<10$ & 200 & N & N \\
\hline LH0368C & 10 & N & 2,000 & 2,000 & 700 & 100 & $<10$ & 500 & N & N \\
\hline LH0369C & 10 & N & $>2,000$ & 1,500 & 300 & 70 & $<10$ & 700 & N & $N$ \\
\hline LH0370C & $<10$ & N & 1,500 & 300 & 200 & 50 & $<10$ & 1,000 & $N$ & N \\
\hline LH0371C & 15 & N & 1,500 & 1,500 & 20 & 70 & 50 & 300 & N & $N$ \\
\hline LH0372C & $<10$ & $N$ & 200 & 1,500 & 50 & 30 & $<10$ & 1,000 & $N$ & $N$ \\
\hline LH0373C & $<10$ & N & 700 & 500 & 50 & 100 & $<10$ & N & N & N \\
\hline LH0374C & $<10$ & $N$ & 700 & 500 & 70 & $<50$ & N & 1,500 & N & N \\
\hline LH0375C & 10 & N & 700 & 300 & 100 & 150 & $<10$ & 70 & $\mathbf{N}$ & N \\
\hline LH0376C & 10 & N & $>2,000$ & 500 & 100 & $<50$ & 30 & 70 & N & $N$ \\
\hline LH0377C & 15 & $N$ & 1,000 & 500 & 150 & 70 & $<10$ & 2,000 & $N$ & N \\
\hline LH0378C & $<10$ & $N$ & N & 150 & 15 & $<50$ & 200 & 500 & $N$ & N \\
\hline Lн0379C & 10 & N & N & 300 & $N$ & $<50$ & 100 & 2,000 & $\mathbf{N}$ & N \\
\hline LH0380C & $<10$ & N & N & 150 & 50 & $<50$ & 30 & $>50,000$ & $N$ & 700 \\
\hline LH0381C & 15 & $N$ & 200 & 200 & 50 & 70 & 150 & 1,500 & $N$ & $<200$ \\
\hline LH0382C & $<10$ & N & 150 & 150 & N & $<50$ & 50 & 200 & N & N \\
\hline LH0383C & $<10$ & $N$ & N & 150 & $N$ & $<50$ & 100 & 2,000 & $\mathbf{N}$ & N \\
\hline LH0384C & $<10$ & $N$ & N & 100 & N & $<50$ & 70 & 1,500 & $\mathbf{N}$ & N \\
\hline LH0385C & N & $N$ & N & 70 & 15 & $<50$ & 30 & 200 & N & $N$ \\
\hline LH0386C & $N$ & $N$ & N & 50 & $N$ & $<50$ & 20 & $<20$ & $\mathbf{N}$ & N \\
\hline LH0387C & $N$ & $\mathbf{N}$ & N & 70 & N & $<50$ & 30 & $<20$ & $\mathbf{N}$ & N \\
\hline LH0388C & N & N & 100 & 70 & 15 & $<50$ & 700 & 7,000 & N & 300 \\
\hline LH0393C & N & N & N & 70 & 15 & $<50$ & 500 & 700 & N & N \\
\hline LH0394C & 10 & N & 300 & 100 & 30 & $<50$ & 500 & 500 & N & N \\
\hline LH0395C & $<10$ & N & 300 & 70 & 20 & $<50$ & 300 & 300 & N & N \\
\hline LH0396C & N & N & 150 & 100 & 15 & $<50$ & 150 & 1,500 & $N$ & N \\
\hline LH0397C & 10 & $N$ & 150 & 150 & 10 & $<50$ & 150 & 2,000 & $N$ & $N$ \\
\hline LH0398C & $N$ & N & N & 70 & N & $<50$ & 100 & 500 & $N$ & $\mathbf{N}$ \\
\hline LH0399C & 15 & N & N & 200 & 15 & $<50$ & 200 & 3,000 & $N$ & N \\
\hline LH0400C & 10 & N & N & 100 & 30 & $<50$ & 30 & 3,000 & $N$ & 300 \\
\hline LH0401C & $<10$ & N & N & 150 & $N$ & $<50$ & 70 & 100 & N & N \\
\hline
\end{tabular}


TABLE 2. GEOChEMICAL DATA FOR NONMAGNETIC, hEAVY-MINERAL-CONCENTRATE SAMPLES, LIME hILLS QUADRANGLE, ALASKA--Cont inUed

Sample Sc ppm-s Sn ppm-s Sr ppm-s Th ppm-s U ppm-s V ppm-s $\quad$ W ppm-s $\quad Y$ ppm-s Zn ppm-s Zr ppm-s

\begin{tabular}{|c|c|c|c|c|c|c|c|c|c|c|}
\hline LH0345C & 20 & 70 & 700 & N & N & 150 & 50 & 200 & 5,000 & $>2,000$ \\
\hline LH0346C & 30 & 1,500 & 200 & 1,000 & 1,000 & 70 & 2,000 & 500 & N & $>2,000$ \\
\hline LH0347C & 20 & 700 & 200 & $N$ & N & 70 & 50 & 300 & $N$ & $>2,000$ \\
\hline LH0348C & 70 & 300 & 200 & $N$ & $N$ & 100 & 2,000 & 300 & $\mathbf{N}$ & $>2,000$ \\
\hline LH0349C & 30 & 300 & $<200$ & N & $N$ & 70 & 150 & 500 & $N$ & $>2,000$ \\
\hline LHO35OC & 20 & $\mathbf{N}$ & 200 & $<200$ & $N$ & 50 & 50 & 300 & $N$ & $>2,000$ \\
\hline LH0351C & 70 & 70 & 300 & 1,500 & N & 100 & 300 & 700 & N & $>2,000$ \\
\hline LH0352C & 50 & 70 & $<200$ & 5,000 & 5,000 & 20 & 15,000 & 700 & N & $>2,000$ \\
\hline LH0353C & 30 & 2,000 & $<200$ & 2,000 & 1,500 & 20 & 700 & 700 & N & $>2,000$ \\
\hline LH0354C & 50 & 1,000 & $<200$ & 1,500 & 1,500 & 20 & 300 & 1,000 & 7,000 & $>2,000$ \\
\hline LH0355C & 30 & 200 & 300 & 500 & 1,500 & 70 & 300 & 1,000 & 2,000 & $>2,000$ \\
\hline LH0356C & 30 & 700 & $<200$ & 700 & 1,000 & $<20$ & 100 & 1,500 & 1,500 & $>2,000$ \\
\hline LH0357C & 70 & 150 & $<200$ & 700 & N & 70 & 50 & 700 & 1,000 & $>2,000$ \\
\hline LH0361C & 10 & $<20$ & $<200$ & N & N & 150 & 20,000 & 150 & N & $>2,000$ \\
\hline LH0362C & 70 & 150 & $<200$ & 1,000 & 1,000 & 30 & 300 & 1,500 & $\mathbf{N}$ & $>2,000$ \\
\hline LH0363C & 200 & 30 & $<200$ & 1,500 & 1,500 & 50 & 2,000 & 2,000 & $N$ & $>2,000$ \\
\hline LH0364C & 150 & 100 & N & 500 & 1,500 & 20 & 300 & 1,500 & $N$ & $>2,000$ \\
\hline LH0365C & 30 & 70 & $<200$ & $<200$ & N & $\mathbf{N}$ & 1,000 & 700 & $N$ & $>2,000$ \\
\hline LH0366C & 30 & N & $\mathbf{N}$ & $<200$ & $\mathbf{N}$ & $\mathbf{N}$ & 1,000 & 700 & $N$ & $>2,000$ \\
\hline LH0367C & 70 & 1,500 & $N$ & 500 & 3,000 & 20 & 3,000 & 700 & $N$ & $>2,000$ \\
\hline LH0368C & 150 & 500 & $<200$ & 1,000 & 1,500 & 150 & 700 & 700 & $N$ & $>2,000$ \\
\hline LH0369C & 150 & 500 & $<200$ & 2,000 & 2,000 & 70 & 1,000 & 1,500 & $\mathbf{N}$ & $>2,000$ \\
\hline LH0370C & 100 & 700 & $<200$ & 1,000 & 5,000 & N & 1,000 & 500 & N & $>2,000$ \\
\hline LH0371C & 70 & 300 & 200 & 300 & N & 150 & 300 & 300 & 20,000 & $>2,000$ \\
\hline LH0372C & 30 & 1,000 & 200 & 200 & N & 50 & 200 & 500 & N & $>2,000$ \\
\hline LH0373C & 20 & 70 & 200 & N & $N$ & 300 & 500 & 300 & $\mathbf{N}$ & $>2,000$ \\
\hline LH0374C & 70 & $>2,000$ & $<200$ & 300 & N & $<20$ & 500 & 700 & 3,000 & $>2,000$ \\
\hline LH0375C & 30 & 100 & 300 & 3,000 & 1,500 & 300 & 1,000 & 300 & N & $>2,000$ \\
\hline LH0376C & 150 & N & $<200$ & 1,000 & 2,000 & 100 & 500 & 700 & N & $>2,000$ \\
\hline LH0377C & 20 & 300 & 700 & 500 & $N$ & 50 & 300 & 1,000 & 5,000 & $>2,000$ \\
\hline LH0378C & $\mathbf{N}$ & $N$ & 1,500 & N & $N$ & 70 & N & 150 & 1,500 & $>2,000$ \\
\hline LH0379C & $<10$ & $N$ & 700 & N & $N$ & 150 & 150 & 70 & 500 & 1,000 \\
\hline LH0380C & N & 30 & 500 & N & $N$ & 100 & 150 & 150 & N & $>2,000$ \\
\hline LH0381C & 15 & 300 & $<200$ & 300 & $N$ & 200 & 2,000 & 700 & 1,000 & $>2,000$ \\
\hline LH0382C & $<10$ & N & 7,000 & N & N & 70 & 50 & 150 & 1,500 & $>2,000$ \\
\hline LH0383C & $<10$ & $N$ & $>10,000$ & 1,500 & 1,500 & 50 & $\mathbf{N}$ & 200 & 1,500 & $>2,000$ \\
\hline LH0384C & N & $N$ & 10,000 & N & N & 50 & $\mathbf{N}$ & 20 & 1,500 & 300 \\
\hline LH0385C & N & N & 3,000 & N & N & $<20$ & N & 30 & N & 50 \\
\hline LH0386C & N & $\mathbf{N}$ & 7,000 & $\mathbf{N}$ & $N$ & $<20$ & N & 20 & 1,500 & 70 \\
\hline LH0387C & $N$ & $\mathbf{N}$ & 7,000 & N & $N$ & 20 & N & 20 & 700 & 50 \\
\hline LH0388C & 30 & 20 & N & $<200$ & $N$ & 20 & 300 & 500 & N & $>2,000$ \\
\hline LH0393C & $N$ & $N$ & 500 & N & N & 70 & N & 50 & 1,000 & 300 \\
\hline LH0394C & N & $N$ & 500 & N & $\mathbf{N}$ & 100 & N & 70 & 700 & 200 \\
\hline LH0395C & $\mathbf{N}$ & N & 700 & $N$ & $N$ & 150 & N & 100 & 700 & 150 \\
\hline LH0396C & 10 & N & 3,000 & N & N & 70 & N & 200 & 1,500 & 700 \\
\hline LH0397C & 10 & $\mathbf{N}$ & 2,000 & 200 & $N$ & 150 & N & 150 & 1,000 & $>2,000$ \\
\hline LH0398C & $N$ & N & 7,000 & N & N & 20 & N & N & 500 & 100 \\
\hline LH0399C & 15 & 70 & 7,000 & $N$ & N & 150 & 50 & 150 & N & $>2,000$ \\
\hline LHO4OOC & $<10$ & 150 & 3,000 & $N$ & N & 100 & N & 150 & 500 & $>2,000$ \\
\hline LHO40IC & $N$ & $N$ & 5,000 & $N$ & $N$ & 70 & $N$ & 70 & 700 & 700 \\
\hline
\end{tabular}




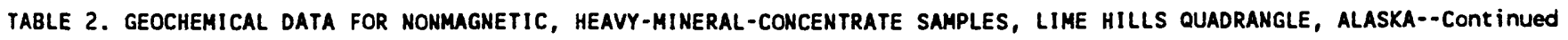

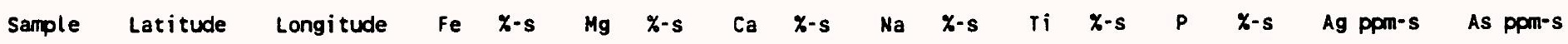

\begin{tabular}{|c|c|c|c|c|c|c|c|c|c|c|c|c|c|}
\hline LH0402C & 6154 & 50 & 154 & & 35 & 10.00 & .70 & 5.00 & .7 & .500 & .5 & 3.0 & N \\
\hline LHO403C & 6153 & 1 & 154 & 0 & 46 & 5.00 & .20 & 20.00 & .7 & .070 & 3.0 & 20.0 & N \\
\hline LH0404C & 6152 & $57^{\circ}$ & 154 & & 40 & 15.00 & .30 & 7.00 & .5 & .150 & .5 & 150.0 & 5,000 \\
\hline LH0406C & 6159 & 0 & 154 & 22 & 56 & .50 & .20 & 3.00 & .5 & .300 & 3.0 & $N$ & N \\
\hline LH0407C & 6156 & 3 & 154 & 17 & 2 & 5.00 & .10 & 15.00 & 1.0 & .100 & 7.0 & 5.0 & N \\
\hline LHO408C & 6159 & 57 & 154 & 13 & 1 & 5.00 & .30 & 5.00 & .7 & .500 & .5 & $N$ & 3,000 \\
\hline LH0409C & 6154 & 28 & 154 & 15 & 2 & 2.00 & .15 & 15.00 & 1.5 & .150 & 10.0 & N & N \\
\hline LH0410C & 6151 & 8 & 154 & 10 & 55 & 15.00 & 1.50 & 7.00 & 1.0 & .150 & N & $N$ & 1,000 \\
\hline LHO4 $11 \mathrm{C}$ & 6149 & 42 & 154 & 5 & 15 & 7.00 & .30 & 10.00 & .7 & .150 & 5.0 & 30.0 & 700 \\
\hline ᄂН0412C & 6146 & 22 & 154 & 3 & 42 & 20.00 & .07 & 5.00 & .7 & 1.000 & 1.5 & 5.0 & 20,000 \\
\hline LHO413C & 6148 & 22 & 154 & 1 & 42 & 15.00 & .20 & 7.00 & .7 & .200 & 3.0 & 150.0 & 1,000 \\
\hline LH0414C & 6159 & 43 & 153 & 2 & 50 & 1.00 & .70 & 7.00 & .5 & .150 & 7.0 & 2.0 & 500 \\
\hline LH0415C & 6157 & 53 & 153 & 0 & 53 & .20 & .20 & 2.00 & $<.5$ & .050 & 3.0 & $N$ & $\mathbf{N}$ \\
\hline LH0416C & 6158 & 4 & 153 & 2 & 58 & 1.00 & .70 & 20.00 & .5 & .700 & 15.0 & $\mathbf{N}$ & N \\
\hline LH0417C & 6154 & 43 & 153 & 4 & 47 & 3.00 & 1.50 & 10.00 & .7 & .700 & 5.0 & 2.0 & 500 \\
\hline LHO418C & 6152 & 51 & 153 & 3 & 58 & 1.00 & .20 & 10.00 & .7 & 2.000 & 7.0 & 2.0 & 500 \\
\hline LH0419C & 6152 & 21 & 153 & 1 & 54 & 5.00 & .30 & 5.00 & .5 & 1.000 & 5.0 & 5.0 & 500 \\
\hline LHO42OC & 6151 & 53 & 153 & 6 & 58 & 2.00 & .30 & 15.00 & .7 & 1.000 & 10.0 & 2.0 & 1,500 \\
\hline LH0421C & 6149 & 39 & 153 & 9 & 15 & 1.00 & .07 & 5.00 & 1.0 & 1.000 & 5.0 & 2.0 & $<500$ \\
\hline LH0422C & 6137 & 7 & 153 & 45 & 36 & 5.00 & $<.05$ & 3.00 & $<.5$ & $>2.000$ & 3.0 & 5.0 & $>20,000$ \\
\hline LH0423C & 6135 & 46 & 153 & 45 & 31 & 5.00 & .05 & 3.00 & $<.5$ & $>2.000$ & 3.0 & 3.0 & 500 \\
\hline LH0424C & 6133 & 49 & 153 & 45 & 40 & 7.00 & .07 & 10.00 & .7 & 1.500 & 5.0 & 10.0 & 3,000 \\
\hline LH0425C & 6132 & 20 & 153 & 45 & 51 & 3.00 & .10 & 5.00 & .7 & 1.000 & 3.0 & 3.0 & 1,000 \\
\hline LH0426C & 6134 & 14 & 153 & 43 & 14 & 7.00 & .07 & 10.00 & .7 & 2.000 & 3.0 & 5.0 & 7,000 \\
\hline LH0427C & 6130 & 53 & 153 & 47 & 47 & .50 & .05 & 1.00 & $\mathbf{N}$ & .200 & 1.0 & $N$ & 1,500 \\
\hline ㄴ0ㄴㄹㄷ & 6129 & 20 & 153 & 43 & 38 & 7.00 & .30 & 5.00 & 1.0 & 1.500 & 2.0 & 70.0 & 10,000 \\
\hline LH0429C & 6128 & 34 & 153 & 45 & 34 & .70 & .10 & 1.50 & $N$ & .200 & 1.0 & $N$ & 500 \\
\hline 내0431C & 6126 & 17 & 153 & 47 & 32 & .70 & .07 & 2.00 & $N$ & .300 & 1.5 & $\mathbf{N}$ & 500 \\
\hline LH0432C & 6125 & 43 & 153 & 42 & 30 & 5.00 & 1.00 & 5.00 & 1.5 & 1.500 & 2.0 & 2.0 & $\mathbf{N}$ \\
\hline LHO433C & 6123 & 21 & 153 & 47 & 6 & 1.50 & .10 & 10.00 & .7 & .300 & 3.0 & N & N \\
\hline LH0434C & 6123 & 26 & 153 & 40 & 55 & 3.00 & .30 & 10.00 & 1.0 & 1.000 & 2.0 & 50.0 & $<500$ \\
\hline LH0435C & 6122 & 14 & 153 & 47 & 39 & .70 & .05 & 5.00 & .5 & 2.000 & 1.5 & $N$ & N \\
\hline LH0436C & 6122 & 47 & 153 & 37 & 31 & .70 & .30 & 20.00 & .7 & 1.500 & 10.0 & $N$ & 500 \\
\hline LH0437C & 6123 & 30 & 153 & 35 & 5 & 1.00 & .50 & 10.00 & .7 & 2.000 & 5.0 & 5.0 & 2,000 \\
\hline 내0438C & 6121 & 33 & 153 & 31 & 46 & 7.00 & .10 & 2.00 & .7 & 2.000 & 3.0 & 20.0 & 1,000 \\
\hline LH0439C & 6121 & 30 & 153 & 31 & 51 & 20.00 & $<.05$ & 2.00 & .7 & 2.000 & 1.5 & 100.0 & 500 \\
\hline LH0440C & 6125 & 7 & 153 & 29 & 19 & 30.00 & $<.05$ & .20 & $<.5$ & .700 & $N$ & 200.0 & 500 \\
\hline Lн0441C & 6125 & 46 & 153 & 28 & 23 & 5.00 & .10 & 10.00 & .7 & $>2.000$ & 5.0 & 10.0 & 15,000 \\
\hline LH0442C & 6125 & 55 & 153 & 27 & 30 & 10.00 & $<.05$ & 2.00 & .5 & $>2.000$ & 1.0 & 10.0 & 1,500 \\
\hline LH0443C & 6125 & 42 & 153 & 27 & 20 & 10.00 & .05 & 1.50 & .7 & 2.000 & .7 & 70.0 & 3,000 \\
\hline LH0444C & 6127 & 32 & 153 & 34 & 41 & 3.00 & .20 & 7.00 & 1.0 & 2.000 & 1.5 & 20.0 & 700 \\
\hline LH0445C & 6127 & 57 & 153 & 34 & 33 & 5.00 & .30 & 10.00 & .7 & 2.000 & 1.5 & 50.0 & 10,000 \\
\hline LH0446C & 6125 & 59 & 153 & 35 & 59 & 5.00 & .50 & 10.00 & 1.0 & 1.000 & 1.0 & 7.0 & 700 \\
\hline LH0447C & 6124 & 35 & 153 & 26 & 1 & 20.00 & $<.05$ & .50 & .7 & 1.500 & .5 & 20.0 & 700 \\
\hline LH0448C & 6119 & 35 & 153 & 38 & 37 & 2.00 & .30 & 7.00 & 1.0 & 1.000 & 3.0 & 5.0 & 500 \\
\hline LH0449C & 6119 & 17 & 153 & 33 & 9 & 5.00 & .10 & 2.00 & 1.5 & 2.000 & 2.0 & 20.0 & $<500$ \\
\hline LH0450C & 6119 & 13 & 153 & 33 & 4 & 1.50 & .07 & 10.00 & 1.0 & $>2.000$ & 7.0 & 10.0 & 500 \\
\hline LH0451C & 6116 & 51 & 153 & 39 & 20 & 3.00 & .20 & 7.00 & 1.5 & .300 & 1.5 & $N$ & $<500$ \\
\hline LH0452C & 6116 & 47 & 153 & 39 & 17 & .70 & .07 & 2.00 & 1.5 & 1.000 & 1.5 & 3.0 & N \\
\hline LH0453C & 6125 & 13 & 153 & 59 & 37 & 3.00 & $<.05$ & 5.00 & .7 & 2.000 & 5.0 & 10.0 & 15,000 \\
\hline
\end{tabular}


TABLE 2. GEOCHEMICAL DATA FOR NONMAGNETIC, hEAVY-MINERAL-CONCENTRATE SAMPLES, LIME hILLS QUAdRANGLE, ALASKA--ContinUEd

Sample AU ppm-s B ppm-s Ba ppm-s Be ppm-s Bi ppm-s cd ppm-s co ppm-s Cr ppm-s Cu ppm-s

\begin{tabular}{|c|c|c|c|c|c|c|c|c|c|}
\hline LHO402C & N & 50 & $>10,000$ & $\mathbf{N}$ & $\mathbf{N}$ & $N$ & 70 & 30 & 700 \\
\hline LHO4O3C & N & N & $>10,000$ & N & N & 50 & 20 & $<20$ & 100 \\
\hline LHO404C & N & 300 & $>10,000$ & N & 20 & $\mathbf{N}$ & 200 & $<20$ & 150 \\
\hline LH0406C & N & 20 & 5,000 & $N$ & N & $N$ & N & $<20$ & 10 \\
\hline LH0407C & N & $\mathbf{N}$ & $>10,000$ & $N$ & $N$ & N & 30 & 20 & 150 \\
\hline LH0408C & N & 20 & $>10,000$ & $N$ & $<20$ & N & 30 & $<20$ & 200 \\
\hline LH0409C & N & 20 & $>10,000$ & $N$ & N & N & 20 & $<20$ & 50 \\
\hline LH0410C & $\mathbf{N}$ & 20 & $>10,000$ & N & $N$ & 50 & 70 & 20 & 50 \\
\hline LH0411C & $\mathbf{N}$ & $\mathbf{N}$ & $>10,000$ & $\mathbf{N}$ & N & 100 & 70 & $<20$ & 100 \\
\hline LH04 12C & $N$ & 20 & 10,000 & $\mathbf{N}$ & N & N & 150 & 30 & 150 \\
\hline LH0413C & $\mathbf{N}$ & 100 & $>10,000$ & $\mathbf{N}$ & 30 & 100 & 150 & $<20$ & 200 \\
\hline LHO414C & $\mathbf{N}$ & 100 & 700 & $\mathbf{N}$ & N & N & $<20$ & 700 & 10 \\
\hline LHO415C & $\mathbf{N}$ & N & 200 & N & $\mathbf{N}$ & $N$ & N & 150 & $<10$ \\
\hline LHO416C & $\mathbf{N}$ & 100 & 500 & N & $N$ & N & $<20$ & 500 & 20 \\
\hline LH0417C & $N$ & N & 5,000 & N & N & $N$ & 20 & 500 & 20 \\
\hline LHO418C & $N$ & $N$ & $>10,000$ & $\mathbf{N}$ & N & N & $<20$ & 200 & 10 \\
\hline LH0419C & $N$ & N & $>10,000$ & $N$ & 150 & 150 & 30 & 200 & 150 \\
\hline LH0420C & $\mathbf{N}$ & 300 & 1,500 & $\mathbf{N}$ & N & $\mathbf{N}$ & $<20$ & 30 & 50 \\
\hline LH0421C & $\mathbf{N}$ & 200 & 3,000 & 2 & N & $\mathbf{N}$ & 20 & 20 & 20 \\
\hline LH0422C & $N$ & N & 200 & 2 & 100 & N & 700 & $<20$ & 100 \\
\hline LH0423C & $N$ & N & $>10,000$ & $\mathbf{N}$ & $\mathbf{N}$ & $\mathbf{N}$ & 70 & 20 & 100 \\
\hline LH0424C & 50 & $\mathbf{N}$ & 2,000 & $\mathbf{N}$ & $N$ & $\mathbf{N}$ & 100 & $<20$ & 150 \\
\hline LH0425C & $\mathbf{N}$ & 30 & 3,000 & 2 & N & $\mathbf{N}$ & 30 & 70 & 70 \\
\hline LH0426C & $N$ & 20 & 700 & $N$ & $N$ & $N$ & 100 & $<20$ & 150 \\
\hline LH0427C & N & 20 & 200 & $\mathbf{N}$ & N & N & 50 & $<20$ & 100 \\
\hline LHO428C & N & 300 & 700 & $\mathbf{N}$ & 1,000 & $N$ & 200 & $<20$ & 500 \\
\hline LH0429C & N & N & 150 & N & N & N & 20 & $<20$ & 50 \\
\hline LH0431C & N & N & 150 & $\mathbf{N}$ & N & $\mathbf{N}$ & 30 & $<20$ & 30 \\
\hline LHO432C & N & 50 & 300 & 2 & 100 & $\mathbf{N}$ & $<20$ & 30 & 150 \\
\hline LHO433C & $\mathbf{N}$ & N & 150 & 2 & N & $N$ & N & $<20$ & 10 \\
\hline LHO434C & $\mathbf{N}$ & 70 & 300 & 2 & 100 & $\mathbf{N}$ & $<20$ & $<20$ & 150 \\
\hline LHO435C & $N$ & N & 150 & N & N & $\mathbf{N}$ & $<20$ & 20 & 10 \\
\hline LH0436C & $N$ & 100 & 300 & $N$ & N & N & $N$ & 50 & 200 \\
\hline LHO437C & N & 1,500 & 150 & $\mathbf{N}$ & 20 & $N$ & 100 & 200 & 200 \\
\hline LH0438C & $\mathbf{N}$ & 100 & 1,000 & 7 & $<20$ & $N$ & $<20$ & $<20$ & 150 \\
\hline LH0439C & $N$ & N & 2,000 & 3 & N & 50 & 20 & $<20$ & 150 \\
\hline LHO $440 \mathrm{C}$ & N & $\mathbf{N}$ & 7,000 & N & N & N & 30 & $<20$ & 100 \\
\hline LH0441C & N & $\mathbf{N}$ & 700 & 2 & 700 & $\mathbf{N}$ & 30 & 50 & 200 \\
\hline LHO442C & N & $N$ & 7,000 & 5 & N & $\mathbf{N}$ & 50 & $<20$ & 150 \\
\hline LH0443C & N & N & 2,000 & 3 & 20 & $N$ & $<20$ & $<20$ & 100 \\
\hline LH0444C & N & 5,000 & 700 & 5 & 150 & N & 50 & 30 & 300 \\
\hline LH0445C & N & $>5,000$ & 200 & 3 & 150 & $N$ & 300 & 30 & 700 \\
\hline LH0446C & N & 2,000 & 300 & 5 & N & 100 & 20 & 30 & 700 \\
\hline LH0447C & N & N & 150 & $N$ & 30 & N & 100 & $<20$ & 200 \\
\hline LHO448C & N & 20 & 7,000 & 2 & 100 & N & $<20$ & 30 & 50 \\
\hline LH0449C & N & 20 & 1,500 & 3 & 100 & N & $<20$ & $<20$ & 200 \\
\hline LH0450C & $N$ & $N$ & 7,000 & 20 & N & 50 & $N$ & $<20$ & 70 \\
\hline LHO451C & $N$ & $N$ & 300 & 2 & $\mathbf{N}$ & N & N & $<20$ & 30 \\
\hline LHO452C & $N$ & N & 300 & 2 & N & N & N & $<20$ & 150 \\
\hline LH0453C & $\mathbf{N}$ & 100 & 70 & 15 & 70 & N & 700 & $<20$ & 200 \\
\hline
\end{tabular}




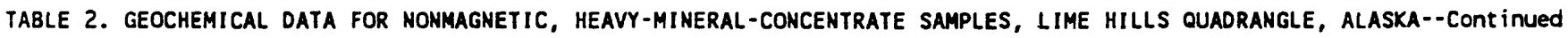

Sample Ga ppm-s Ge ppm-s La ppm-s Mn ppm-s Mo ppm-s Nb ppm-s Ni ppm-s Pb ppm-s Pt ppm-s Sb ppm-s

\begin{tabular}{|c|c|c|c|c|c|c|c|c|c|}
\hline LH0402C & $<10$ & N & 100 & 150 & N & $<50$ & 100 & 1,000 & N \\
\hline LH0403C & $<10$ & N & 100 & 150 & $N$ & $<50$ & 50 & 1,500 & $\mathbf{N}$ \\
\hline LH0404C & 10 & N & N & 100 & $N$ & $<50$ & 200 & 20,000 & N \\
\hline LH0406C & 10 & $N$ & 300 & 150 & N & N & N & 150 & N \\
\hline LH0407C & $<10$ & $N$ & 200 & 150 & 10 & $<50$ & 70 & 1,500 & N \\
\hline LH0408C & $<10$ & $N$ & N & 70 & $N$ & $<50$ & 30 & 20 & N \\
\hline LH0409C & $<10$ & $N$ & 200 & 70 & $N$ & $<50$ & 30 & 300 & $N$ \\
\hline LH0410C & $<10$ & $N$ & $N$ & 70 & $N$ & $<50$ & 50 & 100 & N \\
\hline LH0411C & $<10$ & $\mathbf{N}$ & N & 50 & $N$ & $<50$ & 50 & 7,000 & N \\
\hline LH0412C & $<10$ & $\mathbf{N}$ & 150 & 100 & N & $<50$ & 500 & 500 & N \\
\hline LH0413C & $<10$ & $N$ & N & 150 & $N$ & $<50$ & 150 & $>50,000$ & N \\
\hline LH0414C & $N$ & N & 1,000 & 500 & $N$ & $N$ & 50 & 300 & N \\
\hline LH0415C & $N$ & N & 300 & 150 & N & $N$ & $<10$ & 150 & $N$ \\
\hline LH0416C & N & $N$ & 1,500 & 1,000 & N & $<50$ & 20 & 150 & $N$ \\
\hline LH0417C & $<10$ & $N$ & 500 & 1,500 & N & $<50$ & 20 & 300 & $\mathbf{N}$ \\
\hline LH0418C & $<10$ & $N$ & 500 & 500 & N & $<50$ & 10 & 500 & $\mathbf{N}$ \\
\hline LHO $419 \mathrm{C}$ & $N$ & $N$ & 500 & 500 & $<10$ & $<50$ & 70 & 1,000 & $N$ \\
\hline LH0420C & 10 & N & 700 & 1,500 & 200 & 50 & 10 & 1,500 & $\mathbf{N}$ \\
\hline LH0421C & 10 & $N$ & 200 & 500 & $<10$ & 50 & $<10$ & 150 & $\mathbf{N}$ \\
\hline LH0422C & N & $N$ & 2,000 & 300 & $N$ & 100 & 700 & 150 & $\mathbf{N}$ \\
\hline LH0423C & $N$ & $N$ & N & 100 & 20 & 50 & 70 & 200 & $N$ \\
\hline LH0424C & 10 & N & 300 & 200 & $N$ & $<50$ & 30 & 100 & $N$ \\
\hline LH0425C & 30 & $N$ & 150 & 150 & N & $<50$ & 30 & 300 & N \\
\hline LHO426C & $<10$ & $N$ & 300 & 200 & N & 50 & 20 & 150 & $N$ \\
\hline LH0427C & N & $N$ & 1,000 & 150 & $N$ & N & $<10$ & 30 & $N$ \\
\hline LH0428C & 20 & $N$ & 150 & 700 & 50 & $<50$ & 20 & 5,000 & N \\
\hline LH0429C & $<10$ & $N$ & 1,000 & 150 & $N$ & $N$ & N & 30 & N \\
\hline LH0431C & $<10$ & $N$ & 300 & 150 & $N$ & N & $<10$ & 30 & N \\
\hline LH0432C & 30 & N & 300 & 1,000 & $N$ & 50 & $<10$ & 70 & N \\
\hline LH0433C & 15 & N & 300 & 200 & N & $N$ & $<10$ & 20 & N \\
\hline LH0434C & 20 & N & 150 & 700 & 150 & 50 & $<10$ & 500 & $N$ \\
\hline LH0435C & 10 & $N$ & 200 & 150 & $N$ & $<50$ & $<10$ & 30 & $\mathbf{N}$ \\
\hline LH0436C & 15 & $N$ & 700 & 500 & N & $<50$ & $<10$ & 1,500 & $N$ \\
\hline LH0437C & 10 & N & 300 & 200 & 70 & 50 & 30 & 1,000 & $N$ \\
\hline LH0438C & 10 & N & 300 & 200 & 50 & 100 & $<10$ & 1,000 & N \\
\hline LH0439C & 10 & $N$ & 150 & 150 & 50 & 50 & $<10$ & 1,000 & N \\
\hline LHO44OC & $N$ & $N$ & $N$ & 100 & $N$ & $<50$ & 10 & 200 & N \\
\hline LH0441C & $<10$ & $N$ & 700 & 200 & 70 & 150 & 10 & 1,500 & N \\
\hline LHO442C & N & $\mathbf{N}$ & 300 & 150 & $N$ & 50 & 10 & 500 & N \\
\hline LH0443C & 15 & $N$ & 200 & 200 & N & 50 & $<10$ & 500 & N \\
\hline LH0444C & 30 & N & 150 & 700 & 300 & 50 & $<10$ & 1,500 & N \\
\hline LH0445C & 30 & $N$ & 200 & 1,000 & 300 & 50 & 10 & 10,000 & $N$ \\
\hline LH0446C & 50 & $\mathbf{N}$ & 150 & 1,000 & 70 & $<50$ & $<10$ & 700 & N \\
\hline LH0447C & $<10$ & $\mathbf{N}$ & N & 150 & N & 100 & $<10$ & 1,500 & N \\
\hline LH0448C & 10 & N & 1,500 & 500 & 100 & 50 & $<10$ & 300 & N \\
\hline LH0449C & 10 & $N$ & 300 & 700 & 70 & 100 & $<10$ & 1,500 & N \\
\hline LH0450C & $<10$ & N & 300 & 700 & 50 & 150 & $<10$ & 700 & N \\
\hline LH0451C & 10 & N & 700 & 700 & 10 & $<50$ & $<10$ & 70 & N \\
\hline LH0452C & $<10$ & $N$ & 1,000 & 200 & 20 & $<50$ & $<10$ & 150 & N \\
\hline LH0453C & N & $N$ & $>2,000$ & 1,500 & $N$ & 50 & 1,000 & 200 & $N$ \\
\hline
\end{tabular}


TABLE 2. GEOCHEMICAL DATA FOR NONMAGNETIC, hEAVY-MINERAL-CONCENTRATE SAMPLES, LIME hILLS OUADRANGLE, ALASKA--CONTinUEd

Sample Sc ppm-s Sn ppm-s sr ppm-s Th ppm-s $U$ ppm-s $v$ ppm-s W ppm-s $\quad$ Y ppm-s Zn ppm-s Zr ppm-s

\begin{tabular}{|c|c|c|c|c|c|c|c|c|c|c|}
\hline LHO4O2C & $<10$ & $N$ & 3,000 & $N$ & N & 70 & $N$ & 70 & N & $>2,000$ \\
\hline LH0403C & $<10$ & $N$ & 7,000 & $N$ & N & 70 & $N$ & 150 & 2,000 & 200 \\
\hline LH0404C & $N$ & N & 3,000 & N & N & 50 & 70 & 50 & 500 & $>2,000$ \\
\hline LH0406C & 15 & N & 300 & 200 & N & 70 & $\mathbf{N}$ & 1,000 & N & $>2,000$ \\
\hline LH0407C & 10 & $N$ & 7,000 & $N$ & N & 100 & $\mathbf{N}$ & 200 & N & 2,000 \\
\hline LHO $408 \mathrm{C}$ & $<10$ & 20 & 7,000 & N & N & 50 & N & 70 & 1,500 & $>2,000$ \\
\hline LH0409C & 10 & $\mathbf{N}$ & 7,000 & N & N & 100 & N & 300 & N & $>2,000$ \\
\hline LH0410C & $<10$ & $N$ & $>10,000$ & N & N & 70 & $N$ & $<20$ & 2,000 & 500 \\
\hline LHO411C & $<10$ & N & 7,000 & $\mathbf{N}$ & N & 70 & N & 200 & 5,000 & $>2,000$ \\
\hline LH04 12C & 30 & 30 & 300 & $\mathbf{N}$ & N & 70 & 100 & 500 & N & $>2,000$ \\
\hline LHO413C & 10 & 70 & 1,500 & N & N & 50 & 100 & 150 & 3,000 & $>2,000$ \\
\hline LHO414C & 70 & 150 & 200 & 300 & N & 70 & N & 500 & N & $>2,000$ \\
\hline LH0415C & 70 & $N$ & $<200$ & 200 & N & $<20$ & $N$ & 700 & N & $>2,000$ \\
\hline LH0416C & 70 & 300 & 500 & 200 & N & 70 & 50 & 500 & N & $>2,000$ \\
\hline LH0417C & 50 & 50 & 200 & 200 & N & 100 & 50 & 300 & N & $>2,000$ \\
\hline LH04 18C & 20 & 300 & 700 & $\mathbf{N}$ & N & 70 & $\mathbf{N}$ & 300 & N & $>2,000$ \\
\hline LHO419C & 15 & 20 & 1,500 & $N$ & N & 50 & 70 & 200 & 7,000 & $>2,000$ \\
\hline LH0420C & 20 & $>2,000$ & 500 & 300 & N & 70 & 2,000 & 300 & N & $>2,000$ \\
\hline LH0421C & 15 & $>2,000$ & 200 & 200 & N & 20 & 2,000 & 300 & 2,000 & $>2,000$ \\
\hline LH0422C & 150 & 700 & $<200$ & 300 & 1,000 & 20 & 1,000 & 1,000 & N & $>2,000$ \\
\hline LH0423C & 15 & N & 1,500 & N & N & 50 & N & 150 & $N$ & $>2,000$ \\
\hline LHO424C & 15 & $N$ & 200 & 300 & $N$ & 100 & 150 & 300 & $N$ & $>2,000$ \\
\hline LH0425C & $\mathbf{N}$ & $\mathbf{N}$ & 200 & N & N & 100 & 200 & 150 & $N$ & $>2,000$ \\
\hline LH0426C & 15 & N & 200 & $<200$ & N & 150 & 50 & 300 & $\mathbf{N}$ & $>2,000$ \\
\hline LH0427C & 100 & 50 & N & 7,000 & 5,000 & $<20$ & 100 & 1,000 & $N$ & $>2,000$ \\
\hline LH0428C & 20 & 700 & 300 & 200 & N & 150 & 500 & 200 & N & $>2,000$ \\
\hline LH0429C & 70 & 50 & N & 5,000 & 5,000 & 50 & 50 & 700 & $\mathbf{N}$ & $>2,000$ \\
\hline LH0431C & 50 & N & N & 3,000 & 2,000 & 70 & 100 & 700 & N & $>2,000$ \\
\hline LHO432C & 30 & 150 & $<200$ & N & N & 150 & 150 & 300 & N & $>2,000$ \\
\hline LHO433C & 30 & $N$ & $<200$ & 1.000 & $<1,000$ & 70 & N & 300 & N & $>2,000$ \\
\hline LHO434C & 50 & 150 & 200 & 500 & N & 150 & 5,000 & 300 & N & $>2,000$ \\
\hline LHO435C & 70 & N & $<200$ & 9.500 & $<1,000$ & 70 & N & 700 & N & $>2,000$ \\
\hline LH0436C & 20 & N & 300 & N & N & 150 & 100 & 500 & $N$ & $>2,000$ \\
\hline LH0437C & 30 & 30 & $<200$ & 200 & $\mathbf{N}$ & 150 & 300 & 300 & $\mathbf{N}$ & $>2,000$ \\
\hline LH0438C & 70 & 200 & N & $N$ & N & 50 & $N$ & 500 & 3,000 & $>2,000$ \\
\hline LH0439C & 50 & 300 & N & N & $N$ & 20 & 50 & 500 & 10,000 & $>2,000$ \\
\hline LHO $440 \mathrm{C}$ & 10 & N & N & N & N & $<20$ & N & 100 & N & $>2,000$ \\
\hline LH044IC & 70 & 150 & $<200$ & $N$ & N & 70 & 100 & 500 & 1,500 & $>2,000$ \\
\hline LH0442C & 70 & 50 & N & $\mathbf{N}$ & N & 150 & 50 & 300 & N & $>2,000$ \\
\hline LH0443C & 50 & 200 & N & N & N & 50 & N & 300 & N & $>2,000$ \\
\hline LH0444C & 30 & 150 & $<200$ & N & N & 150 & 300 & 950 & N & $>2,000$ \\
\hline LH0445C & 50 & 150 & 200 & N & N & 500 & 300 & 300 & 700 & $>2,000$ \\
\hline LHO446C & 30 & 70 & 200 & $\mathbf{N}$ & $N$ & 150 & 100 & 150 & 2,000 & $>2,000$ \\
\hline LHO447C & 20 & N & N & $N$ & $\mathbf{N}$ & N & 50 & 100 & 2,000 & $>2,000$ \\
\hline LH0448C & 50 & 300 & $<200$ & 200 & $\mathbf{N}$ & 70 & 10,000 & 300 & N & $>2,000$ \\
\hline LH0449C & 50 & 1,000 & $<200$ & N & $N$ & 30 & 200 & 300 & 1,500 & $>2,000$ \\
\hline LH0450C & 70 & 30 & 300 & N & N & 20 & 50 & 500 & 7,000 & $>2,000$ \\
\hline LH0451C & 20 & $N$ & 500 & 3,000 & 1,500 & 100 & 2,000 & 300 & $N$ & $>2,000$ \\
\hline LH0452C & 50 & 30 & $<200$ & 1,000 & 1,500 & 20 & 700 & 500 & $N$ & $>2,000$ \\
\hline LH0453C & 200 & $>2,000$ & N & 500 & 3,000 & 20 & 200 & 1,500 & $\mathbf{N}$ & $>2,000$ \\
\hline
\end{tabular}


TABLE 2. GEOCHEMICAL DATA FOR NONMAGNETIC, heAVY-MINERAL-CONCENTRATE SAMPLES, LIME hILLS QUADRANGLE, ALASKa--ContinUed

\begin{tabular}{|c|c|c|c|c|c|c|c|c|c|c|}
\hline Sample & Lat itude & Longi tude & X-s & $x-s$ & $x-s$ & \%-s & $x-s$ & $x-s$ & Ag ppm-s & As ppm \\
\hline LHO454C & $\begin{array}{lll}61 & 25 & 18\end{array}$ & 1535929 & 3.00 & .10 & 10.00 & .5 & 1.000 & 7.0 & 7.0 & 1,51 \\
\hline LH0455C & 612327 & 1535523 & 3.00 & .15 & 15.00 & .7 & 1.000 & 10.0 & 10.0 & 3,01 \\
\hline LH0456C & 612329 & $153 \quad 55 \quad 11$ & 3.00 & .07 & 10.00 & .7 & 1.500 & 7.0 & 200.0 & 5,01 \\
\hline LH0457C & 612513 & 1535331 & 15.00 & .05 & 5.00 & .7 & 1.500 & 5.0 & 500.0 & 10,01 \\
\hline LH0458C & 612643 & 1535356 & 7.00 & .07 & 10.00 & .7 & 1.500 & 7.0 & 300.0 & 15,08 \\
\hline LHO459C & 612249 & 153543 & .70 & .10 & 2.00 & 1.0 & .300 & 1.0 & $N$ & \\
\hline LHO460C & 612031 & 1535336 & 3.00 & .10 & 15.00 & .7 & $>2.000$ & 10.0 & 5.0 & 10,00 \\
\hline LHO $461 \mathrm{C}$ & 612030 & 1535048 & 3.00 & .05 & 15.00 & 1.0 & $>2.000$ & 7.0 & 30.0 & $>20,00$ \\
\hline LHO462C & 61205 & 1534845 & 3.00 & .20 & 10.00 & .7 & .300 & 1.0 & $N$ & 1,00 \\
\hline LHO463C & 611910 & 1535628 & 1.00 & .05 & 20.00 & .7 & $>2.000$ & 20.0 & 10.0 & 2,06 \\
\hline LHO464C & 611715 & 1535218 & 1.00 & .07 & 20.00 & 1.5 & 1.500 & 7.0 & $N$ & \\
\hline LHO $465 \mathrm{C}$ & 611634 & 1534845 & 1.00 & .07 & 20.00 & 1.0 & $>2.000$ & 15.0 & $N$ & 50 \\
\hline LH0466C & 615648 & $\begin{array}{lll}15327 & 2\end{array}$ & 7.00 & 1.00 & 3.00 & 1.0 & 1.500 & 3.0 & 20.0 & 7.00 \\
\hline LHO $467 \mathrm{C}$ & 615643 & $\begin{array}{lll}153 & 27 & 1\end{array}$ & 10.00 & .30 & 7.00 & .7 & .700 & 5.0 & 20.0 & $5,0 C$ \\
\hline LHO468C & 615752 & 153322 & 1.00 & .15 & 20.00 & 1.0 & $>2.000$ & 7.0 & 20.0 & \\
\hline LH0469C & 615246 & $\begin{array}{lll}153 & 13 & 27\end{array}$ & 7.00 & 2.00 & 3.00 & 1.0 & $>2.000$ & 1.5 & $N$ & 76 \\
\hline LHO5OOC & 614723 & $154 \quad 410$ & 20.00 & 1.50 & 5.00 & .7 & .300 & 2.0 & 3.0 & $2,0 \mathrm{C}$ \\
\hline LH0501C & 614532 & 154223 & 20.00 & .30 & 5.00 & .5 & .300 & .5 & 3.0 & 3,00 \\
\hline LH0508C & 613526 & 1531630 & 10.00 & .50 & 7.00 & 1.0 & .700 & 1.5 & 30.0 & $7.0 \mathrm{C}$ \\
\hline LH0509C & 613746 & 1531520 & 15.00 & .30 & 5.00 & 1.0 & 1.500 & 1.5 & 20.0 & $3,0 \mathrm{C}$ \\
\hline LH0510C & 614229 & $\begin{array}{lll}153 & 10 & 17\end{array}$ & 3.00 & 1.50 & 10.00 & 1.0 & 1.500 & 2.0 & 2.0 & $1,0 c$ \\
\hline LH0511C & $\begin{array}{llll}61 & 41 & 1\end{array}$ & $\begin{array}{lll}153 & 12 & 7\end{array}$ & 2.00 & .20 & 20.00 & .7 & $>2.000$ & 20.0 & 10.0 & $2,0 \mathrm{C}$ \\
\hline LH0512C & 61437 & $153 \quad 8 \quad 28$ & 5.00 & 1.00 & 15.00 & 1.5 & 1.500 & 2.0 & 15.0 & \\
\hline LH0513C & 614451 & $\begin{array}{lll}153 & 6 & 8\end{array}$ & 1.00 & .20 & 2.00 & 1.0 & 1.000 & 3.0 & 7.0 & $1,0 \mathrm{C}$ \\
\hline LHOS14C & 614748 & 153510 & 7.00 & 1.00 & 3.00 & 1.0 & .700 & 3.0 & 200.0 & $5,0 C$ \\
\hline LH0515C & 614550 & 153927 & 7.00 & 3.00 & 7.00 & 1.0 & 1.500 & 5.0 & 20.0 & 1,50 \\
\hline LH05 16C & 614539 & $153 \quad 1233$ & 10.00 & .20 & 7.00 & .5 & 1.500 & 3.0 & 300.0 & 20,00 \\
\hline LH05 17C & 614723 & $153 \quad 15 \quad 53$ & 15.00 & .20 & 2.00 & $<.5$ & 1.500 & 1.5 & 70.0 & $>20,0 \mathrm{C}$ \\
\hline LH060OC & 615511 & $154 \quad 1952$ & 7.00 & 2.00 & 15.00 & 1.0 & .200 & $N$ & 1.0 & \\
\hline LH0601C & 615754 & $15414 \quad 26$ & 20.00 & .20 & 7.00 & .7 & .150 & 5.0 & 2.0 & \\
\hline LH0602C & 615939 & $154 \quad 14 \quad 12$ & 30.00 & .30 & 2.00 & .5 & .070 & $N$ & $<1.0$ & \\
\hline LH0603C & 615333 & $154 \quad 1341$ & 20.00 & .20 & 3.00 & .7 & .200 & 1.5 & 2.0 & 1,50 \\
\hline LHO604C & 615038 & $\begin{array}{lll}154 & 8 & 7\end{array}$ & 20.00 & 1.50 & 7.00 & 1.0 & .200 & $N$ & 1.0 & 3,00 \\
\hline LH0605C & 615024 & $\begin{array}{lll}154 & 7\end{array}$ & 7.00 & 1.00 & 7.00 & .7 & .300 & .7 & 10.0 & \\
\hline LH0606C & 614712 & $154 \quad 234$ & 15.00 & .50 & 7.00 & .7 & .300 & 3.0 & 20.0 & 50 \\
\hline LHO6OTC & 614526 & 1535853 & 30.00 & .70 & 5.00 & .5 & .200 & $N$ & 100.0 & 1,50 \\
\hline LHO608C & 615923 & 153859 & 5.00 & .10 & 10.00 & .7 & 2.000 & 7.0 & $N$ & \\
\hline LHO609C & 615837 & 153927 & 7.00 & .50 & 7.00 & .7 & $>2.000$ & 5.0 & $N$ & \\
\hline LHO610C & 615654 & 153950 & 2.00 & .15 & 7.00 & $<.5$ & $>2.000$ & 3.0 & $N$ & \\
\hline LHO611C & 615543 & 153414 & .70 & .10 & 20.00 & 1.0 & 2.000 & $>20.0$ & $N$ & \\
\hline LHO612C & 615132 & 153119 & 5.00 & .15 & 15.00 & .7 & $>2.000$ & 7.0 & 2.0 & $<50$ \\
\hline LH0613C & 615132 & 15315 & 1.00 & .30 & 15.00 & $<.5$ & .300 & 7.0 & 7.0 & 3,00 \\
\hline LH0614C & 615127 & 153458 & 3.00 & .70 & 20.00 & .7 & .150 & $>20.0$ & 70.0 & \\
\hline LH0615C & 614734 & 153912 & 7.00 & 1.50 & 15.00 & .7 & 1.500 & 10.0 & 15.0 & 70 \\
\hline LH0616C & 613629 & 1534550 & 5.00 & $<.05$ & 2.00 & $<.5$ & $>2.000$ & 7.0 & 70.0 & $>20,00$ \\
\hline LH0617C & 613659 & 1534836 & 5.00 & .07 & 5.00 & .5 & $>2.000$ & 5.0 & 20.0 & $>20,00$ \\
\hline LH0618C & 613634 & $153 \quad 48 \quad 42$ & 1.00 & .07 & 10.00 & .7 & $>2.000$ & 10.0 & 3.0 & 2,00 \\
\hline LH0619C & 613451 & $\begin{array}{lll}153 & 47 & 1\end{array}$ & 2.00 & $<.05$ & 5.00 & .5 & $>2.000$ & 7.0 & 5.0 & 10,00 \\
\hline LHO62OC & 61319 & 1534511 & 1.00 & .15 & .70 & .5 & .200 & .7 & $N$ & 1,00 \\
\hline LH0621C & 613231 & 1535016 & 1.00 & .07 & 10.00 & $<.5$ & $>2.000$ & 10.0 & 5.0 & 5,00 \\
\hline
\end{tabular}


TABLE 2. GEOCHEMICAL DATA FOR NONMAGNETIC, HEAVY-MINERAL-CONCENTRATE SAMPLES, LIME HILLS QUADRANGLE, ALASKA--CONTINUEd

Sample AU ppm-s B ppm-s Ba ppm-s Be ppm-s Bi ppm-s Co cd ppm-s Co ppm-s Cr ppm-s Cu ppm-s

\begin{tabular}{|c|c|c|c|c|c|c|c|c|c|}
\hline LHO454C & $N$ & 70 & 300 & 3 & $<20$ & $N$ & 100 & 100 & 150 \\
\hline LH0455C & N & 300 & 500 & 3 & 70 & $N$ & 300 & 70 & 200 \\
\hline LHO456C & $\mathbf{N}$ & 200 & 300 & 2 & 100 & N & 100 & 30 & 200 \\
\hline LHO457C & $N$ & 50 & 150 & N & 70 & N & 500 & 30 & 200 \\
\hline LHO $458 \mathrm{C}$ & $N$ & 500 & 150 & $N$ & 50 & N & 500 & 30 & 200 \\
\hline LH0459C & N & 100 & 500 & 5 & $N$ & $N$ & 30 & $<20$ & 30 \\
\hline LHO460C & N & 500 & 150 & 5 & 50 & $N$ & 500 & $<20$ & 150 \\
\hline LHO461C & $N$ & 300 & 150 & 3 & 150 & N & 700 & $<20$ & 300 \\
\hline LHO462C & $\mathbf{N}$ & 700 & 700 & 7 & $\mathbf{N}$ & $N$ & 70 & 30 & 10 \\
\hline LH0463C & $N$ & 150 & 70 & 3 & 200 & $\mathbf{N}$ & 150 & $<20$ & 100 \\
\hline LH0464C & N & 700 & 150 & 3 & 150 & $N$ & $<20$ & $<20$ & 100 \\
\hline LH0465C & $N$ & 200 & 300 & 3 & 30 & $\mathbf{N}$ & 70 & $<20$ & 50 \\
\hline LHO466C & N & 5,000 & $>10,000$ & 3 & 200 & $N$ & 70 & 70 & 1,000 \\
\hline LHO467C & $N$ & 300 & $>10,000$ & $N$ & 70 & $N$ & 100 & 30 & 300 \\
\hline LHO468C & $N$ & 20 & 700 & $N$ & $N$ & $N$ & $<20$ & 70 & 15 \\
\hline LHO469C & $N$ & 50 & 1,500 & 5 & 30 & $N$ & 30 & 300 & 100 \\
\hline LHO50OC & $N$ & 200 & $>10,000$ & 20 & $N$ & $N$ & 100 & 50 & 150 \\
\hline LH0501C & $\mathbf{N}$ & $N$ & $>10,000$ & N & $\mathbf{N}$ & $N$ & 100 & 30 & 300 \\
\hline LH0508C & $N$ & 100 & 2,000 & 5 & 70 & N & 100 & 70 & 1,000 \\
\hline LH0509C & $N$ & N & $>10,000$ & $N$ & 30 & N & 30 & 50 & 200 \\
\hline LH0510C & N & 300 & 500 & 50 & 2,000 & $N$ & $<20$ & 200 & 50 \\
\hline LHO511C & $N$ & 70 & 300 & 3 & $N$ & N & 30 & 100 & 200 \\
\hline LHO512C & N & 700 & 700 & 10 & N & $N$ & $<20$ & 70 & 50 \\
\hline LH0513C & $N$ & N & 700 & 5 & 300 & $N$ & $<20$ & 30 & 50 \\
\hline LHOS14C & 70 & 1,500 & 3,000 & 15 & 20 & N & 50 & 70 & 2,000 \\
\hline LH0515C & $N$ & 20 & 700 & 2 & $\mathbf{N}$ & $N$ & 50 & 300 & 300 \\
\hline LH0516C & 70 & 70 & 5,000 & 7 & 200 & 50 & 70 & 20 & 700 \\
\hline LHOS17C & $N$ & 100 & $>10,000$ & $N$ & 200 & $N$ & 100 & 30 & 150 \\
\hline LH0600C & N & 500 & $>10,000$ & N & $N$ & $N$ & 30 & 150 & 300 \\
\hline LH0601C & N & 70 & $>10,000$ & N & $\mathbf{N}$ & $\mathbf{N}$ & 100 & 20 & 500 \\
\hline LH0602C & N & $N$ & $>10,000$ & N & $N$ & 50 & 100 & $<20$ & 300 \\
\hline LHO603C & $N$ & $\mathbf{N}$ & $>10,000$ & N & $\mathrm{N}$ & $N$ & 70 & $<20$ & 500 \\
\hline LH0604C & $N$ & 20 & $>10,000$ & N & N & N & 100 & 30 & 150 \\
\hline LHO605C & $N$ & 30 & $>10,000$ & N & N & $N$ & 30 & 30 & 150 \\
\hline LH0606C & $N$ & 70 & $>10,000$ & N & N & $N$ & 100 & 20 & 200 \\
\hline LH0607C & N & 150 & 700 & N & $N$ & $N$ & 500 & 20 & 150 \\
\hline LHO608C & $N$ & $N$ & $>10,000$ & N & $N$ & $N$ & $<20$ & 50 & 100 \\
\hline LH0609C & $N$ & 100 & $>10,000$ & 3 & $\mathbf{N}$ & $N$ & 50 & 200 & 700 \\
\hline LH0610C & $N$ & $N$ & $>10,000$ & N & N & N & $<20$ & 30 & 150 \\
\hline LH0611C & N & $N$ & 300 & $N$ & $\mathbf{N}$ & N & $<20$ & 30 & 10 \\
\hline LHO612C & N & 70 & $>10,000$ & $N$ & $\mathbf{N}$ & N & 50 & 300 & 200 \\
\hline LH0613C & $N$ & 100 & $>10,000$ & $N$ & 30 & $\mathbf{N}$ & 150 & 300 & 30 \\
\hline LHO614C & 70 & $N$ & 500 & $N$ & N & $N$ & 20 & 30 & 30 \\
\hline LH0615C & $N$ & 150 & 1,000 & 3 & N & $N$ & 70 & 50 & 100 \\
\hline LH0616C & N & $N$ & 700 & 2 & 200 & N & 700 & $<20$ & 150 \\
\hline LHO617C & $N$ & 100 & 500 & 10 & 300 & $\mathbf{N}$ & 70 & $<20$ & 150 \\
\hline LH0618C & $\mathbf{N}$ & 500 & 150 & 3 & 30 & $N$ & 30 & $<20$ & 100 \\
\hline LH0619C & N & $N$ & 150 & 3 & 30 & $N$ & 300 & $<20$ & 30 \\
\hline LHO620C & $N$ & 100 & 1,000 & 5 & $N$ & N & $<20$ & 100 & 30 \\
\hline LH0621C & $N$ & 500 & 300 & N & 30 & N & 150 & $<20$ & 200 \\
\hline
\end{tabular}


TABLE 2. GEOCHEMICAL DATA FOR NONMAgNETIC, hEAVY-MINERAL-CONCENTRATE SAMPLES, LIME hILLS QUADRANGLE, ALASKA--Cont inUEd Sample Ga ppm-s Ge ppm-s La ppm-s Mn ppm-s Mo ppm-s Nb ppm-s Ni ppm-s Pb ppm-s Pt ppm-s Sb ppm-s

\begin{tabular}{|c|c|c|c|c|c|c|c|c|}
\hline LHO454C & 30 & $N$ & 300 & 2,000 & 20 & $<50$ & 100 & 100 \\
\hline LH0455C & 20 & $N$ & 1,500 & 3,000 & 50 & $<50$ & 300 & 500 \\
\hline LH0456C & 10 & $\mathbf{N}$ & 2,000 & 1,000 & $N$ & $<50$ & 70 & 500 \\
\hline LH0457C & 10 & $N$ & $>2,000$ & 500 & 30 & $<50$ & 500 & 3,000 \\
\hline LH0458C & 15 & $N$ & $>2,000$ & 700 & $N$ & $<50$ & 700 & 5,000 \\
\hline LHO459C & 20 & $N$ & 500 & 200 & $N$ & $N$ & $<10$ & 100 \\
\hline LH0460C & 10 & N & 1,000 & 3,000 & $N$ & 100 & 300 & 70 \\
\hline LH0461C & $<10$ & $\mathbf{N}$ & 1,500 & 3,000 & $\mathbf{N}$ & 100 & 500 & 70 \\
\hline LH0462C & 30 & $N$ & 2,000 & 1,000 & $\mathbf{N}$ & N & 20 & 20 \\
\hline LH0463C & $<10$ & $N$ & 1,000 & 3,000 & N & 100 & 100 & 200 \\
\hline LH0464C & 15 & $\mathbf{N}$ & 1.500 & 3,000 & N & 70 & $<10$ & 30 \\
\hline LH0465C & 10 & $N$ & 700 & 7,000 & N & 70 & 50 & 150 \\
\hline LH0466C & 30 & $\mathbf{N}$ & 700 & 700 & $N$ & 50 & 70 & 200 \\
\hline LHO467C & $<10$ & $\mathbf{N}$ & 200 & 500 & N & $<50$ & 200 & 3,000 \\
\hline LHO $468 \mathrm{C}$ & $<10$ & $N$ & 700 & 200 & $\mathbf{N}$ & $<50$ & $<10$ & 30 \\
\hline LH0469C & 30 & $\mathbf{N}$ & 500 & 3,000 & 10 & 50 & 50 & 150 \\
\hline LHOSOOC & 15 & $\mathbf{N}$ & $N$ & 200 & $\mathbf{N}$ & $<50$ & 150 & 150 \\
\hline LHOSO1C & 10 & $\mathbf{N}$ & $N$ & 150 & $N$ & $<50$ & 300 & 500 \\
\hline LH0508C & 70 & $\mathbf{N}$ & 1,500 & 1,000 & $\mathbf{N}$ & $<50$ & 50 & 7,000 \\
\hline LH0509C & 20 & $N$ & 150 & 500 & $\mathbf{N}$ & $<50$ & 20 & 5,000 \\
\hline LHOS 10C & 20 & $N$ & 500 & 1,000 & 15 & 70 & 20 & 200 \\
\hline LH0511C & 10 & $N$ & 1,000 & 1,000 & $N$ & 70 & 20 & 500 \\
\hline LH0512C & 30 & $N$ & 700 & 1,500 & 20 & 70 & 10 & 5,000 \\
\hline LH0513C & 10 & N & 1,500 & 500 & 700 & 100 & $<10$ & 5,000 \\
\hline LH05 14C & 15 & N & 300 & 2,000 & 30 & $<50$ & 70 & 2,000 \\
\hline LHOS15C & 15 & $\mathbf{N}$ & 700 & 3,000 & 50 & 70 & 50 & 2,000 \\
\hline LHOS16C & 10 & $N$ & 300 & 1,500 & 700 & 100 & 50 & $>50,000$ \\
\hline LHOS17C & 10 & $N$ & 200 & 200 & $\mathbf{N}$ & $<50$ & 100 & 7,000 \\
\hline LHO60OC & 20 & $\mathbf{N}$ & $\mathbf{N}$ & 300 & $\mathbf{N}$ & $<50$ & 70 & 700 \\
\hline LH0601C & $<10$ & $\mathbf{N}$ & 150 & 150 & 20 & $<50$ & 300 & 70 \\
\hline LH0602C & $<10$ & $N$ & $\mathbf{N}$ & 50 & $N$ & $<50$ & 200 & 50 \\
\hline LH0603C & $<10$ & $\mathbf{N}$ & $N$ & 100 & 20 & $<50$ & 500 & 70 \\
\hline LH0604C & 10 & $N$ & $N$ & 150 & $\mathbf{N}$ & $<50$ & 200 & 200 \\
\hline LH0605C & $<10$ & N & 150 & 150 & 50 & $<50$ & 50 & 2,000 \\
\hline LH0606C & $<10$ & N & $\mathbf{N}$ & 150 & $\mathbf{N}$ & $<50$ & 300 & 2,000 \\
\hline LH0607C & 10 & $\mathbf{N}$ & N & 150 & N & $<50$ & 500 & 15,000 \\
\hline LHO6OBC & 10 & $\mathbf{N}$ & 300 & 500 & N & $<50$ & 30 & 300 \\
\hline LH0609C & 15 & $\mathbf{N}$ & 700 & 1,500 & $N$ & 50 & 70 & 150 \\
\hline LHO610C & N & $N$ & 150 & 300 & N & 50 & 10 & 150 \\
\hline LHO611C & $<10$ & $N$ & 700 & 1,500 & N & $<50$ & $<10$ & $\mathbf{N}$ \\
\hline LH0612C & $<10$ & $N$ & 500 & 2,000 & N & 50 & 30 & 200 \\
\hline LH0613C & $<10$ & $N$ & 1,000 & 300 & $N$ & $<50$ & 50 & 700 \\
\hline LH0614C & $<10$ & $\mathbf{N}$ & 700 & 1,500 & 20 & $<50$ & $<10$ & 3,000 \\
\hline LH0615C & 20 & $\mathbf{N}$ & 700 & 1,500 & 100 & 50 & 30 & 3,000 \\
\hline LH0616C & $\mathbf{N}$ & $N$ & 200 & 700 & $N$ & 200 & 500 & 700 \\
\hline LH0617C & 20 & $N$ & 300 & 500 & $\mathbf{N}$ & 200 & 20 & 150 \\
\hline LH0618C & 10 & $\mathbf{N}$ & 1,000 & 2,000 & 20 & 150 & $<10$ & 100 \\
\hline LH0619C & $<10$ & $N$ & 200 & 500 & $N$ & 150 & 200 & 500 \\
\hline LHO620C & 30 & $N$ & N & 100 & $N$ & $<50$ & 10 & $N$ \\
\hline LH0621C & 10 & $\mathbf{N}$ & 700 & 1,000 & $\mathbf{N}$ & 100 & 50 & 100 \\
\hline
\end{tabular}




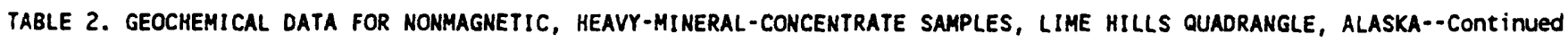

Sample Sc ppm-s Sn ppm-s sr ppm-s Th ppm-s $U$ ppm-s $V$ ppm-s $\quad$ W ppm-s $\quad$ Y ppm-s Zn ppm-s Zr ppm.

\begin{tabular}{|c|c|c|c|c|c|c|c|c|c|c|}
\hline LHO454C & 70 & 50 & $<200$ & $N$ & $N$ & 150 & 50 & 700 & $N$ & $>2,001$ \\
\hline LH0455C & 150 & $>2,000$ & $<200$ & $<200$ & N & 70 & 100 & 1,500 & N & $>2,001$ \\
\hline LHO456C & 150 & 1,000 & $<200$ & 2,000 & 2,000 & 70 & 150 & 1,000 & N & $>2,000$ \\
\hline LH0457C & 150 & 1,500 & $<200$ & 2,000 & 3,000 & 70 & 500 & 1,000 & N & $>2,000$ \\
\hline LH0458C & 200 & 1,500 & $<200$ & 1.500 & 3,000 & 70 & 100 & 1,500 & N & $>2,00 \mathrm{C}$ \\
\hline LH0459C & 70 & 30 & $<200$ & $>5,000$ & 5,000 & 50 & N & 1,000 & N & $>2,000$ \\
\hline LHO460C & 150 & $>2,000$ & $<200$ & 200 & 1,000 & 30 & 100 & 1,500 & $N$ & $>2,00 \mathrm{C}$ \\
\hline LH0461C & 150 & $>2,000$ & $<200$ & 200 & N & 20 & 300 & 1,000 & N & $>2,00 \mathrm{C}$ \\
\hline LH0462C & 150 & 30 & $<200$ & 2,000 & 1,000 & 100 & $N$ & 700 & N & $>2,000$ \\
\hline LH0463C & 150 & $>2,000$ & $<200$ & N & $N$ & 30 & 150 & 1,000 & N & $>2,000$ \\
\hline LH0464C & 100 & 2,000 & $<200$ & $<200$ & $<1,000$ & $<20$ & 300 & 1,500 & $N$ & $>2,00 \mathrm{C}$ \\
\hline LH0465C & 100 & $>2,000$ & $<200$ & N & $<1,000$ & 20 & 70 & 1,500 & N & $>2,00 \mathrm{C}$ \\
\hline LH0466C & 20 & $>2,000$ & 200 & $N$ & N & 150 & 300 & 200 & $\mathbf{N}$ & $>2,000$ \\
\hline LH0467C & 15 & 1,000 & 1,000 & N & N & 70 & 200 & 200 & $N$ & $>2,000$ \\
\hline LHO468C & 50 & N & 200 & N & $N$ & 200 & N & 1,000 & $N$ & $>2,000$ \\
\hline LHO469C & 70 & 20 & $<200$ & $<200$ & $N$ & 150 & $N$ & 500 & N & $>2,000$ \\
\hline LH0500C & 10 & N & 500 & N & $N$ & 70 & 50 & 70 & 1,000 & 300 \\
\hline LH0501C & $<10$ & N & 500 & N & $N$ & 70 & N & 30 & 700 & 200 \\
\hline LH0508C & 30 & 700 & 300 & 500 & $N$ & 100 & 150 & 300 & 1,000 & $>2,000$ \\
\hline LH0509C & 30 & 100 & N & N & N & 100 & N & 150 & $N$ & $>2,000$ \\
\hline LH05 10C & 50 & $>2,000$ & 200 & 1,000 & 1,000 & 100 & 200 & 1,500 & $N$ & $>2,000$ \\
\hline LH0511C & 30 & $>2,000$ & 700 & 300 & N & 150 & 200 & 700 & $N$ & $>2,000$ \\
\hline LH05 12C & 50 & $>2,000$ & 500 & 1,000 & $<1,000$ & 150 & 300 & 1,000 & N & $>2,000$ \\
\hline LH0513C & 70 & $>2,000$ & $<200$ & 3,000 & 1,500 & 70 & 15,000 & 1,000 & N & $>2,000$ \\
\hline LH0514C & 30 & $>2,000$ & 200 & N & N & 150 & 3,000 & 300 & $N$ & $>2,000$ \\
\hline LH05 15C & 70 & 200 & 300 & N & N & 150 & 200 & 200 & N & $>2,000$ \\
\hline LH0516C & 20 & $>2,000$ & 200 & 700 & 1,000 & 70 & $>20,000$ & 700 & 3,000 & $>2,000$ \\
\hline LH0517C & 15 & 1,000 & 500 & $N$ & N & 70 & 300 & 150 & 500 & $>2,000$ \\
\hline LH0600C & 15 & N & 3,000 & N & N & 100 & H & 30 & 500 & 300 \\
\hline LH0601C & $<10$ & $N$ & 2,000 & 300 & N & 70 & N & 300 & 1,500 & $>2,000$ \\
\hline LH0602C & N & $N$ & 7,000 & N & $N$ & N & N & $\mathrm{N}$ & 5,000 & 200 \\
\hline LH0603C & $<10$ & N & 1,500 & N & $N$ & 70 & N & 70 & 700 & 200 \\
\hline LH0604C & 10 & N & $>10,000$ & $N$ & N & 70 & N & 50 & 2,000 & $>2,000$ \\
\hline LH0605C & 15 & N & 7,000 & 300 & $N$ & 70 & 300 & 200 & 1,500 & $>2,000$ \\
\hline LH0606C & $<10$ & N & 2,000 & N & $N$ & 70 & N & 100 & 1,000 & 300 \\
\hline LH0607C & $<10$ & 30 & 700 & $<200$ & N & 50 & 2,000 & 50 & 700 & 1,000 \\
\hline LH0608C & 15 & N & 1,000 & N & N & 70 & N & 300 & 1,500 & $>2,000$ \\
\hline LH0609C & 30 & $N$ & 1,000 & $N$ & N & 200 & N & 300 & 700 & $>2,000$ \\
\hline LH0610C & 15 & N & 5,000 & $N$ & N & 70 & $N$ & 300 & 1,500 & $>2,000$ \\
\hline LH0611C & 15 & 300 & 1,000 & N & N & 70 & $N$ & 300 & N & $>2,000$ \\
\hline LH0612C & 20 & $N$ & 1,500 & N & $N$ & 150 & $N$ & 200 & 1,500 & $>2,000$ \\
\hline LH0613C & 30 & 200 & 2,000 & $<200$ & N & 70 & 300 & 500 & N & $>2,000$ \\
\hline LH0614C & 30 & 1.500 & 700 & N & N & 70 & 500 & 200 & $N$ & $>2,000$ \\
\hline LHO615C & 50 & $>2,000$ & 700 & 200 & $N$ & 200 & 150 & 300 & N & $>2,000$ \\
\hline LH0616C & 100 & 500 & N & $<200$ & N & 50 & 3,000 & 700 & $N$ & $>2,000$ \\
\hline LH0617C & 100 & 2,000 & $<200$ & N & N & 70 & 3,000 & 300 & $N$ & $>2,000$ \\
\hline LH0618C & 150 & 150 & $<200$ & $<200$ & $N$ & 50 & 300 & 700 & $N$ & $>2,000$ \\
\hline LH0619C & 150 & 700 & $<200$ & N & N & 70 & 1,000 & 700 & $N$ & $>2,000$ \\
\hline LH0620C & N & N & $<200$ & N & N & 150 & 150 & 50 & $N$ & $>2,000$ \\
\hline LH0621C & 200 & 200 & $<200$ & $N$ & $N$ & 70 & 500 & 700 & $N$ & $>2,000$ \\
\hline
\end{tabular}


TABLE 2. GEOCHEMICAL DATA fOR NONMAGNETIC, hEAVY-MINERAL-CONCENTRATE SAMPLES, LIME HILLS QUADRANGLE, ALASKA--CONTINUEd

\begin{tabular}{|c|c|c|c|c|c|c|c|c|c|c|c|c|c|}
\hline Sample & Latitu & ude & Long & gitur & ude & $x-s$ & $x-s$ & $x-s$ & \%-s & x-s & z-s & Ag ppm-s & As ppm-s \\
\hline HO622C & 6132 & 5 & 153 & 51 & 10 & 2.00 & $<.05$ & 2.00 & $<.5$ & $>2.000$ & 3.0 & 7.0 & 3,000 \\
\hline LH0623C & 6131 & 50 & 153 & 51 & 16 & 2.00 & .05 & 3.00 & $<.5$ & 2.000 & 5.0 & 20.0 & $>20,000$ \\
\hline LHO624C & 6126 & 53 & 153 & 44 & 1 & 3.00 & .30 & 5.00 & .7 & $>2.000$ & 5.0 & 5.0 & 3,000 \\
\hline LH0625C & 6126 & 25 & 153 & 42 & 7 & 5.00 & .30 & 5.00 & 1.0 & $>2.000$ & 3.0 & $\mathbf{N}$ & 10,000 \\
\hline LH0626C & 6124 & 26 & 153 & 46 & 1 & .70 & .10 & 10.00 & .7 & .700 & 10.0 & N & 1 \\
\hline LHO628C & 6125 & 14 & 153 & 59 & 19 & 7.00 & .15 & 1.50 & .5 & .200 & 3.0 & 10.0 & 500 \\
\hline LH0629C & 6123 & 31 & 153 & 565 & 59 & 2.00 & $<.05$ & 15.00 & .7 & $>2.000$ & 7.0 & 5.0 & 10,006 \\
\hline LHO630C & 6128 & 56 & 153 & 55 & 58 & 2.00 & $<.05$ & 20.00 & .7 & .300 & $>20.0$ & 50.0 & 506 \\
\hline H0631C & 6128 & 45 & 153 & 55 & 13 & 5.00 & .05 & 20.00 & .7 & $>2.000$ & 20.0 & 300.0 & 5,001 \\
\hline LH0632C & 6128 & 17 & 153 & 543 & 36 & 3.00 & .20 & 7.00 & .7 & $>2.000$ & 7.0 & 10.0 & A \\
\hline LH0633C & 6122 & 42 & 153 & 543 & 30 & .50 & $<.05$ & 20.00 & .7 & $>2.000$ & 5.0 & N & $1.50 \mathrm{C}$ \\
\hline LH0634C & 6121 & 2 & 153 & 51 & 6 & 1.00 & $<.05$ & 7.00 & 1.0 & .150 & .5 & $N$ & A \\
\hline LH0635C & 6117 & 51 & 153 & 50 & 14 & 3.00 & .30 & 30.00 & 1.0 & 1.500 & 20.0 & $N$ & A \\
\hline LHO636C & 6117 & 11 & 153 & 53 & 27 & 3.00 & .50 & 20.00 & 1.0 & .700 & 20.0 & 10.0 & 10,000 \\
\hline LH0637C & 6117 & 17 & 153 & 53 & 29 & 2.00 & .07 & 20.00 & .7 & $>2.000$ & 20.0 & 3.0 & 3,001 \\
\hline LH0638C & 6118 & 57 & 153 & 43 & 16 & .30 & $<.05$ & 2.00 & $<.5$ & .150 & 3.0 & $N$ & A \\
\hline $.40643 \mathrm{C}$ & 6156 & 47 & 153 & 26 & 19 & 15.00 & 1.00 & 7.00 & .7 & .700 & 5.0 & 2.0 & \\
\hline HO6 $44 \mathrm{C}$ & 6157 & 33 & 153 & 30 & 49 & 10.00 & 1.50 & 3.00 & .7 & 2.000 & 3.0 & 20.0 & 5,000 \\
\hline L $0645 \mathrm{C}$ & 6157 & 29 & 153 & 30 & 57 & 15.00 & .50 & 3.00 & .5 & .700 & 3.0 & 300.0 & 7.000 \\
\hline.$H 0646 \mathrm{C}$ & 6151 & 48 & 153 & 11 & 43 & 1.50 & .10 & 3.00 & .5 & 1.000 & 5.0 & 100.0 & $1,50 C$ \\
\hline LHO647C & 6151 & 2 & 153 & 15 & 16 & .50 & .07 & 3.00 & $<.5$ & .500 & 3.0 & 20.0 & 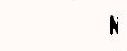 \\
\hline LHO70OC & 6144 & 41 & 153 & 14 & 7 & 7.00 & 1.00 & 7.00 & 1.0 & 2.000 & 5.0 & 7.0 & N \\
\hline Ho701C & 6146 & 5 & 153 & 53 & 38 & 1.00 & .10 & 10.00 & 1.5 & 1.000 & 5.0 & 15.0 & $70 c$ \\
\hline H0702C & 6149 & 10 & 153 & 23 & 35 & 5.00 & .10 & 10.00 & .7 & $>2.000$ & 7.0 & 200.0 & $50 \mathrm{C}$ \\
\hline H0703C & 6149 & 2 & 153 & 2 & 47 & 20.00 & .30 & 5.00 & .7 & 1.000 & 3.0 & 700.0 & $1,50 c$ \\
\hline. $\mathrm{HOTO4C}$ & 6146 & 50 & 153 & 10 & 17 & 10.00 & 1.00 & 7.00 & 1.0 & 1.000 & 3.0 & 200.0 & $5,00 C$ \\
\hline $.40705 \mathrm{C}$ & 6145 & 59 & 153 & 133 & 34 & 5.00 & 1.50 & 15.00 & 1.5 & 1.000 & 7.0 & N & \\
\hline.$H 0706 \mathrm{C}$ & 6147 & 51 & 153 & 194 & 47 & 1.00 & .10 & 15.00 & $<.5$ & .700 & 20.0 & $N$ & N \\
\hline $\mathrm{HOBOOC}$ & 6152 & 7 & 153 & 53 & 46 & 7.00 & .07 & 1.50 & $<.5$ & 1.500 & 1.5 & 20.0 & $>20,000$ \\
\hline $.40801 C$ & 6151 & 48 & 153 & 53 & 27 & 3.00 & .10 & 1.50 & $<.5$ & 1.000 & 1.0 & 7.0 & 15,000 \\
\hline $.40802 C$ & 6150 & 56 & 153 & 525 & 55 & 15.00 & $<.05$ & 2.00 & $<.5$ & .200 & $N$ & 20.0 & $>20,000$ \\
\hline $.40803 \mathrm{C}$ & 6150 & 50 & 153 & 525 & 54 & 2.00 & $<.05$ & 15.00 & .5 & 1.000 & $<.5$ & 20.0 & 10,000 \\
\hline $.40804 \mathrm{C}$ & 6151 & 5 & 154 & 174 & 43 & 30.00 & 1.00 & 5.00 & .7 & .300 & $N$ & 5.0 & 1,500 \\
\hline H0805C & 6156 & 3 & 153 & 295 & 53 & 7.00 & .50 & 10.00 & $<.5$ & $>2.000$ & 7.0 & 3.0 & $N$ \\
\hline H0819C & 6145 & 12 & 153 & 393 & 35 & 30.00 & .15 & .50 & $<.5$ & .150 & $N$ & 20.0 & $>20,000$ \\
\hline $.40820 \mathrm{C}$ & 6145 & 14 & 153 & 39 & 10 & 20.00 & .10 & 1.50 & $<.5$ & .200 & 1.0 & 100.0 & $>20,000$ \\
\hline. $\mathrm{HOB25C}$ & 6145 & 52 & 153 & 373 & 32 & 30.00 & .15 & 1.50 & $<.5$ & 1.500 & 1.5 & 10.0 & $>20,000$ \\
\hline H0830C & 6150 & 28 & 154 & 21 & 13 & 20.00 & .30 & 3.00 & .5 & 2.000 & 1.0 & 7.0 & 3,000 \\
\hline H0831C & 6150 & 39 & 154 & 26 & 4 & 20.00 & .30 & 3.00 & .5 & 2.000 & 1.5 & $N$ & N \\
\hline H0832C & 6150 & 45 & 154 & 26 & 4 & 15.00 & .30 & 3.00 & .5 & 2.000 & 1.0 & N & 3,000 \\
\hline $.40833 C$ & 6150 & 34 & 154 & 273 & 31 & 5.00 & .20 & 2.00 & $<.5$ & .150 & $N$ & $\mathbf{N}$ & $N$ \\
\hline. $\mathrm{H} 0834 \mathrm{C}$ & 6150 & 31 & 154 & 28 & 22 & 3.00 & .20 & 1.50 & $<.5$ & .200 & $<.5$ & $N$ & $<500$ \\
\hline. $\mathrm{HOB35C}$ & 6148 & 9 & 154 & 28 & 9 & 2.00 & .20 & .50 & $<.5$ & .050 & $<.5$ & N & $\mathbf{N}$ \\
\hline $.40836 C$ & 6148 & 14 & 154 & 28 & 5 & 2.00 & .20 & 1.50 & $<.5$ & .150 & $N$ & N & N \\
\hline $.40837 \mathrm{C}$ & 6122 & 9 & 154 & 1 & 26 & 7.00 & .05 & 15.00 & .7 & 2.000 & 7.0 & 20.0 & 7,000 \\
\hline. $\mathrm{H} 0838 \mathrm{C}$ & 6122 & 2 & 154 & 2 & 2 & 2.00 & .10 & 20.00 & .7 & $>2.000$ & 7.0 & 5.0 & 3,000 \\
\hline H0839C & 6119 & 46 & 154 & 0 & 29 & 15.00 & $<.05$ & 10.00 & $<.5$ & 1.000 & 5.0 & 200.0 & $>20,000$ \\
\hline. $\mathrm{H} 0840 \mathrm{C}$ & 6119 & 51 & 154 & 03 & 37 & 2.00 & $<.05$ & 15.00 & $<.5$ & 2.000 & 5.0 & 200.0 & 10,000 \\
\hline $.40841 \mathrm{C}$ & 6118 & 36 & 154 & $13:$ & 32 & 1.00 & .07 & 20.00 & $<.5$ & $>2.000$ & 7.0 & N & 500 \\
\hline. $\mathrm{H} 0842 \mathrm{C}$ & 6115 & 34 & 154 & 16 & 15 & 1.00 & .10 & 7.00 & .5 & $>2.000$ & 5.0 & 2.0 & 2,000 \\
\hline
\end{tabular}




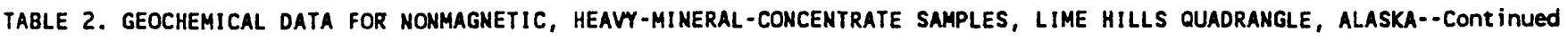
Sample AU ppm-s B ppm-s Ba ppm-s Be ppm-s Bi ppm-s cd ppm-s Co ppm-s Cr ppm-s Cu ppm-:

\begin{tabular}{|c|c|c|c|c|c|c|c|c|c|}
\hline LH0622C & N & $N$ & 150 & 3 & 30 & N & 200 & $<20$ & 200 \\
\hline LH0623C & N & 100 & 300 & 2 & N & N & 700 & $<20$ & 70 \\
\hline LH0624C & $N$ & 20 & 500 & 3 & 100 & $N$ & 30 & 30 & 700 \\
\hline LH0625C & $N$ & 150 & 500 & 7 & N & N & 20 & 20 & 200 \\
\hline LH0626C & $N$ & 50 & 300 & $N$ & $N$ & $N$ & N & $<20$ & 200 \\
\hline LH0628C & N & 150 & 500 & 5 & N & $N$ & 30 & 70 & 50 \\
\hline LH0629C & N & 70 & 200 & 5 & 70 & $N$ & 700 & $<20$ & 100 \\
\hline LH0630C & $N$ & $N$ & 70 & $N$ & $N$ & $N$ & 20 & $<20$ & 20 \\
\hline LH0631C & $N$ & N & 150 & $N$ & 30 & $N$ & $<20$ & $<20$ & 100 \\
\hline LH0632C & $N$ & 2,000 & 300 & 3 & $N$ & $N$ & 50 & 30 & 300 \\
\hline LH0633C & N & 50 & 150 & 100 & 50 & N & 100 & $<20$ & 10 \\
\hline LH0634C & $N$ & 20 & 500 & 5 & $N$ & $N$ & 30 & $<20$ & 150 \\
\hline LH0635C & $N$ & 3,000 & 200 & 3 & N & $N$ & $<20$ & 20 & 700 \\
\hline LH0636C & $N$ & 5,000 & 500 & 3 & 500 & $N$ & 300 & 100 & 150 \\
\hline LH0637C & $N$ & 1,000 & 150 & 10 & 20 & $N$ & 100 & 20 & 100 \\
\hline LH0638C & $N$ & 20 & 300 & $N$ & $N$ & $N$ & N & $<20$ & $<10$ \\
\hline LH0643C & N & 100 & $>10,000$ & $\mathbf{N}$ & N & $N$ & 100 & 70 & 500 \\
\hline LH0644C & $N$ & $>5,000$ & 10,000 & 2 & 500 & $N$ & 300 & 150 & 1,000 \\
\hline LH0645C & N & 1,500 & $>10,000$ & $N$ & 700 & $N$ & 300 & 30 & 1,000 \\
\hline LH0646C & 50 & 100 & 700 & $N$ & N & $N$ & 30 & 100 & 10 \\
\hline LH0647C & 30 & 50 & 300 & $N$ & $N$ & N & $N$ & 100 & $<10$ \\
\hline LHO700C & 20 & 700 & 3,000 & 3 & N & $N$ & 20 & 50 & 50 \\
\hline LH0701C & $N$ & 200 & 700 & 5 & 1,500 & N & 30 & 50 & 150 \\
\hline LH0702C & 20 & 100 & $>10,000$ & $N$ & N & $N$ & 30 & 70 & 200 \\
\hline LH0703C & 50 & 300 & $>10,000$ & $N$ & 20 & $N$ & 100 & 300 & 700 \\
\hline LH0704C & $N$ & 100 & 1,000 & 3 & 20 & N & 50 & 150 & 700 \\
\hline LH0705C & $N$ & 500 & 300 & 7 & $N$ & $N$ & $<20$ & 100 & 50 \\
\hline LH0706C & $N$ & 100 & 700 & N & $<20$ & $N$ & N & 70 & 10 \\
\hline LH0800C & $N$ & $N$ & 150 & $N$ & 200 & $N$ & 3,000 & 150 & 150 \\
\hline LH0801C & $N$ & 50 & 1,500 & $N$ & 200 & $N$ & 150 & 50 & 150 \\
\hline LH0802C & $N$ & $N$ & 70 & 10 & 1,000 & $N$ & 200 & $<20$ & 2,000 \\
\hline LH0803C & $N$ & $N$ & 70 & 7 & 150 & N & 70 & $<20$ & 70 \\
\hline LH0804C & $N$ & 30 & $>10,000$ & N & N & $N$ & 200 & 30 & 100 \\
\hline LH0805C & $N$ & 150 & 500 & 2 & 30 & $N$ & 30 & 150 & 150 \\
\hline LH0819C & N & $N$ & $>10,000$ & N & $N$ & $N$ & 200 & 30 & 150 \\
\hline LH0820C & 500 & $N$ & $>10,000$ & $N$ & N & $N$ & 50 & 30 & 100 \\
\hline LH0825C & N & 20 & 10,000 & $N$ & 200 & $N$ & 1,000 & 100 & 200 \\
\hline LH0830C & $N$ & 30 & $>10,000$ & $N$ & 20 & $N$ & 150 & 70 & 150 \\
\hline LH0831C & $N$ & 150 & $>10,000$ & N & N & 50 & 70 & 70 & 200 \\
\hline LH0832C & $N$ & 20 & $>10,000$ & 3 & N & $N$ & 150 & 70 & 150 \\
\hline LH0833C & $N$ & 100 & $>10,000$ & $N$ & N & $N$ & $<20$ & 50 & 50 \\
\hline LH0834C & $N$ & 30 & $>10,000$ & $N$ & N & $N$ & $<20$ & $<20$ & 50 \\
\hline LH0835C & $N$ & $N$ & $>10,000$ & $N$ & $N$ & $N$ & $<20$ & 150 & 150 \\
\hline LH0836C & $N$ & 20 & $>10,000$ & $N$ & $N$ & N & $<20$ & 30 & 50 \\
\hline LH0837C & $N$ & 70 & $>10,000$ & 30 & 500 & $N$ & 300 & $<20$ & 70 \\
\hline LH0838C & N & 50 & 1,000 & 15 & 100 & $N$ & 70 & $<20$ & 200 \\
\hline LH0839C & $N$ & 20 & 200 & 3 & 200 & N & 1,000 & $<20$ & 100 \\
\hline LH0840C & $N$ & N & 300 & 7 & 150 & N & 700 & $<20$ & 70 \\
\hline LH0841C & $N$ & 50 & 200 & 2 & 30 & N & 20 & 20 & 10 \\
\hline LH0842C & $N$ & 500 & 700 & 15 & 30 & $N$ & 70 & 20 & $<10$ \\
\hline
\end{tabular}




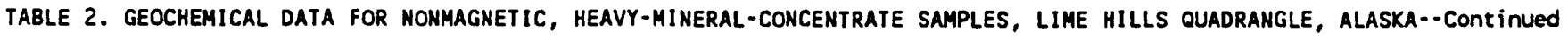

Sample Ga ppm-s Ge ppm-s La ppm-s Mn ppm-s Mo ppm-s Nb ppm-s Ni ppm-s Pb ppm-s Pt ppm-s Sb ppm-s

\begin{tabular}{|c|c|c|c|c|c|c|c|c|c|}
\hline LH0622C & $<10$ & $N$ & 500 & 300 & $N$ & 200 & 150 & 1,000 & N \\
\hline LH0623C & $<10$ & $N$ & 1,500 & 500 & N & $<50$ & 500 & 2,000 & $N$ \\
\hline LH0624C & 20 & $N$ & 300 & 1,000 & 200 & 150 & 10 & 700 & $N$ \\
\hline LH0625C & 70 & N & 300 & 1,000 & 20 & 100 & $<10$ & 100 & N \\
\hline LH0626C & 10 & $N$ & 700 & 300 & $N$ & $<50$ & $<10$ & 70 & N \\
\hline LH0628C & 50 & $N$ & N & 200 & $N$ & $<50$ & 70 & 1,500 & $N$ \\
\hline LH0629C & 10 & $N$ & 500 & 2,000 & N & 100 & 700 & 70 & $N$ \\
\hline LH0630C & $<10$ & $N$ & 300 & 1,500 & 100 & $<50$ & $<10$ & 300 & $N$ \\
\hline LH0631C & $<10$ & N & 500 & 1,000 & 70 & 50 & $<10$ & 10,000 & $N$ \\
\hline LH0632C & 20 & $N$ & $>2,000$ & 1,000 & N & 70 & 20 & 150 & N \\
\hline LH0633C & 10 & $N$ & 500 & 3,000 & N & 150 & 50 & 30 & $N$ \\
\hline LH0634C & 50 & $N$ & N & 300 & N & $N$ & $<10$ & 200 & $N$ \\
\hline LH0635C & 15 & $N$ & 1,500 & 7,000 & N & 50 & $<10$ & 30 & $N$ \\
\hline LH0636C & 30 & N & 1,500 & 5,000 & $N$ & 50 & 150 & 70 & $N$ \\
\hline LH0637C & 15 & $N$ & 1,500 & 5,000 & $N$ & 150 & 50 & 70 & N \\
\hline LH0638C & $<10$ & $N$ & 300 & 200 & $N$ & N & $N$ & $<20$ & $N$ \\
\hline LH0643C & 15 & $N$ & 200 & 1,000 & $N$ & $<50$ & 150 & 2,000 & N \\
\hline LH0644C & 30 & $N$ & 300 & 500 & $N$ & 50 & 300 & 700 & N \\
\hline LH0645C & 10 & $N$ & 200 & 300 & $N$ & $<50$ & 300 & 15,000 & N \\
\hline LH0646C & 30 & $N$ & 200 & 150 & $N$ & 50 & 10 & 200 & N \\
\hline LH0647C & 20 & N & 150 & 150 & $N$ & $<50$ & $<10$ & N & N \\
\hline LHOTOOC & 20 & $N$ & 300 & 1,000 & $N$ & 70 & 10 & 100 & $N$ \\
\hline LH0701C & 20 & N & 300 & 700 & 200 & 50 & 20 & 1,500 & N \\
\hline LH0702C & $<10$ & $N$ & 300 & 700 & 150 & 70 & 30 & 15,000 & $N$ \\
\hline LH0703C & 10 & $N$ & 300 & 1,500 & $N$ & 50 & 150 & 20,000 & $N$ \\
\hline LH0704C & 30 & N & 500 & 3,000 & 70 & 50 & 30 & 10,000 & N \\
\hline LH0705C & 20 & $N$ & 500 & 1,500 & N & 70 & 10 & 100 & N \\
\hline LH0706C & 20 & $N$ & 300 & 200 & $N$ & $<50$ & $<10$ & 50 & $N$ \\
\hline LH0800C & $<10$ & $N$ & 200 & 150 & 15 & 50 & 1,500 & 2,000 & $N$ \\
\hline LH0801C & $<10$ & $N$ & 300 & 150 & 200 & 70 & 50 & 150 & N \\
\hline LH0802C & $<10$ & $N$ & 100 & 100 & 300 & $<50$ & $<10$ & 1,000 & $N$ \\
\hline LH0803C & $<10$ & N & 300 & 150 & 300 & 70 & N & 2,000 & N \\
\hline LH0804C & $<10$ & $N$ & 100 & 150 & $N$ & $<50$ & 150 & 700 & N \\
\hline LH0805C & 15 & $N$ & 700 & 1,000 & $N$ & 50 & 20 & 300 & $N$ \\
\hline LH0819C & $<10$ & $N$ & $N$ & 200 & $N$ & $<50$ & 500 & 200 & N \\
\hline LH0820C & $<10$ & $N$ & N & 200 & $N$ & $<50$ & 70 & 70 & N \\
\hline LH0825C & $<10$ & N & 150 & 300 & $N$ & $<50$ & 1,000 & 200 & $N$ \\
\hline LH0830C & $<10$ & $N$ & 150 & 50 & N & $<50$ & 500 & 1,000 & N \\
\hline LH0831C & $<10$ & $N$ & 150 & 100 & $N$ & 50 & 300 & 500 & N \\
\hline LH0832C & $<10$ & $N$ & 100 & 70 & N & $<50$ & 150 & 500 & N \\
\hline LH0833C & N & N & N & 70 & N & $<50$ & 30 & N & N \\
\hline LH0834C & $<10$ & $N$ & N & 70 & $<10$ & $<50$ & 30 & 20 & N \\
\hline LH0835C & N & $N$ & N & 100 & N & $<50$ & 20 & N & $N$ \\
\hline LH0836C & N & $N$ & $N$ & 100 & $N$ & $<50$ & 20 & $N$ & N \\
\hline LH0837C & 10 & $N$ & 200 & 3,000 & $N$ & 100 & 300 & 100 & $N$ \\
\hline LH0838C & 20 & N & 700 & 5,000 & 50 & 100 & 100 & 30 & $N$ \\
\hline LH0839C & $<10$ & $N$ & 150 & 3,000 & $N$ & 100 & 1,000 & 300 & N \\
\hline LH084OC & $<10$ & $N$ & 500 & 3,000 & $N$ & 70 & 1,000 & 20 & N \\
\hline LH0841C & $<10$ & $N$ & 300 & 5,000 & $N$ & 150 & 10 & 50 & N \\
\hline LH0842C & 10 & $<20$ & 300 & 2,000 & $N$ & 500 & 30 & 30 & $N$ \\
\hline
\end{tabular}




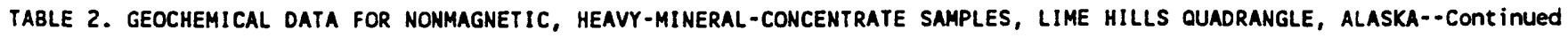

Sample sc ppm-s Sn ppm-s sr ppm-s Th ppm-s $U$ ppm-s $v$ ppm-s W ppm-s $\quad$ Y ppm-s $2 n$ ppm-s $2 r$ ppm-s

\begin{tabular}{|c|c|c|c|c|c|c|c|c|c|c|}
\hline LH0622C & 200 & 150 & $<200$ & N & $\mathbf{N}$ & 100 & 300 & 500 & $N$ & $>2,000$ \\
\hline LH0623C & 150 & 70 & N & 2,000 & 3,000 & 70 & 300 & 1,000 & N & $>2,000$ \\
\hline LH0624C & 30 & 500 & 200 & N & N & 200 & 20,000 & 300 & $N$ & $>2,000$ \\
\hline LH0625C & 30 & 300 & 200 & $\mathbf{N}$ & $N$ & 150 & 500 & 200 & $\mathbf{N}$ & $>2,000$ \\
\hline LHO626C & 30 & $N$ & $<200$ & 300 & $<1,000$ & 70 & 200 & 700 & N & $>2,000$ \\
\hline LH0628C & N & 20 & N & N & N & 100 & N & 150 & 500 & $>2,000$ \\
\hline LH0629C & 150 & $>2,000$ & $<200$ & $\mathbf{N}$ & $N$ & 20 & 500 & 700 & N & $>2,000$ \\
\hline LH0630C & 20 & 20 & 200 & $N$ & $\mathbf{N}$ & 20 & N & 1,000 & $N$ & $>2,000$ \\
\hline LH0631C & 50 & 1,500 & 200 & $\mathbf{N}$ & N & 70 & 500 & 1,000 & $N$ & $>2,000$ \\
\hline LH0632C & 200 & 30 & $<200$ & 1,500 & 1,000 & 100 & N & 700 & $N$ & $>2,000$ \\
\hline LH0633C & 100 & $>2,000$ & $<200$ & N & N & 30 & 200 & 1,000 & $N$ & $>2,000$ \\
\hline LH0634C & 30 & N & $<200$ & 1,000 & $<1,000$ & 50 & 300 & 500 & N & $>2,000$ \\
\hline LH0635C & 70 & 1,500 & $<200$ & 200 & N & 70 & N & 1,500 & N & $>2,000$ \\
\hline LH0636C & 70 & $>2,000$ & $<200$ & 300 & 2,000 & 70 & 150 & 1,500 & N & $>2,000$ \\
\hline LH0637C & 100 & $>2,000$ & $<200$ & 200 & 1,000 & 70 & 150 & 1,500 & N & $>2,000$ \\
\hline LH0638C & 70 & 20 & $<200$ & 500 & 1,500 & 20 & 2,000 & 700 & N & $>2,000$ \\
\hline LHO643C & 20 & 200 & 500 & $N$ & N & 150 & N & 200 & 2,000 & $>2,000$ \\
\hline LH0644C & 30 & 700 & 1,000 & N & $\mathbf{N}$ & 300 & N & 200 & N & $>2,000$ \\
\hline LH0645C & 10 & $>2,000$ & 1,500 & $N$ & $\mathbf{N}$ & 70 & N & 150 & 1,000 & $>2,000$ \\
\hline LH0646C & 15 & 1,000 & $<200$ & N & $\mathbf{N}$ & 150 & 500 & 300 & N & $>2,000$ \\
\hline LH0647C & 15 & 500 & $<200$ & N & $N$ & 150 & 300 & 300 & N & $>2,000$ \\
\hline LH0700C & 20 & 1,500 & 200 & 200 & $\mathbf{N}$ & 200 & N & 300 & $N$ & $>2,000$ \\
\hline LH0701C & 30 & $>2,000$ & 200 & 200 & $N$ & 150 & 10,000 & 500 & N & $>2,000$ \\
\hline LH0702C & 20 & 2,000 & 700 & 200 & N & 150 & 300 & 300 & 2,000 & $>2,000$ \\
\hline LH0703C & 15 & $>2,000$ & 500 & N & $\mathbf{N}$ & 100 & 200 & 200 & 1,500 & $>2,000$ \\
\hline LHO704C & 30 & 2,000 & 300 & $<200$ & $\mathbf{N}$ & 200 & 2,000 & 300 & 1,500 & $>2,000$ \\
\hline LH0705C & 30 & 1,500 & 500 & 700 & $N$ & 200 & 100 & 700 & N & $>2,000$ \\
\hline LH0706C & 10 & 700 & 300 & N & N & 150 & 300 & 500 & N & $>2,000$ \\
\hline LH0800C & 20 & 70 & $N$ & $>5,000$ & 7,000 & 150 & 10,000 & 500 & 1,000 & $>2,000$ \\
\hline LH0801C & 30 & 150 & N & $>5,000$ & 7,000 & 50 & 10,000 & 700 & N & $>2,000$ \\
\hline LHOBO2C & 10 & 1,000 & $N$ & 2,000 & 3,000 & $N$ & 1,000 & 500 & N & $>2,000$ \\
\hline LH0803C & 20 & $>2,000$ & $\mathbf{N}$ & $>5,000$ & 7,000 & 20 & 1,000 & 700 & N & $>2,000$ \\
\hline LH0804C & $\mathbf{N}$ & 150 & 700 & N & N & 100 & N & 70 & 500 & 2,000 \\
\hline LH0805C & 70 & 20 & 300 & N & N & 150 & 100 & 500 & N & $>2,000$ \\
\hline LH0819C & $N$ & $N$ & N & $N$ & $N$ & 70 & 1,000 & 30 & $\mathbf{N}$ & 500 \\
\hline LHOB2OC & $\mathbf{N}$ & $\mathbf{N}$ & 1,500 & $\mathbf{N}$ & $N$ & 50 & 5,000 & 70 & $\mathbf{N}$ & 500 \\
\hline LH0825C & 15 & $\mathbf{N}$ & N & $\mathbf{N}$ & $N$ & 150 & 200 & 150 & N & $>2,000$ \\
\hline LH0830C & 10 & 200 & 2,000 & $\mathbf{N}$ & $\mathbf{N}$ & 100 & N & 100 & 500 & $>2,000$ \\
\hline LH0831C & 15 & 20 & 1,500 & $\mathbf{N}$ & $N$ & 150 & $\mathbf{N}$ & 150 & 3,000 & $>2,000$ \\
\hline LH0832C & 10 & 200 & 5,000 & $\mathbf{N}$ & $\mathbf{N}$ & 100 & $N$ & 100 & 2,000 & $>2,000$ \\
\hline LH0833C & N & N & 5,000 & $N$ & $N$ & $<20$ & $N$ & 30 & N & 1,000 \\
\hline LH0834C & $\mathbf{N}$ & 200 & 5,000 & N & $\mathbf{N}$ & 50 & $N$ & 50 & 1,000 & 2,000 \\
\hline LH0835C & $N$ & 200 & 3,000 & $N$ & $N$ & 20 & $N$ & $<20$ & N & 200 \\
\hline LH0836C & $N$ & N & 7,000 & $N$ & N & 20 & N & $<20$ & $<500$ & 500 \\
\hline LH0837C & 70 & $>2,000$ & $<200$ & $\mathbf{N}$ & $\mathbf{N}$ & 20 & 500 & 700 & $N$ & $>2,000$ \\
\hline LH0838C & 70 & $>2,000$ & $<200$ & $N$ & $\mathbf{N}$ & 20 & 100 & 1,000 & $\mathbf{N}$ & $>2,000$ \\
\hline LH0839C & 50 & $>2,000$ & N & $\mathbf{N}$ & $<1,000$ & $<20$ & 200 & 700 & $\mathbf{N}$ & $>2,000$ \\
\hline LH0840C & 150 & $>2,000$ & $N$ & $\mathbf{N}$ & 1,000 & $<20$ & 70 & 700 & $\mathbf{N}$ & $>2,000$ \\
\hline LH0841C & 100 & $>2,000$ & $N$ & $\mathbf{N}$ & N & 30 & 70 & 1,000 & $N$ & $>2,000$ \\
\hline LH0842C & 70 & $>2,000$ & $N$ & $\mathbf{N}$ & $\mathbf{N}$ & 20 & 100 & 700 & $\mathbf{N}$ & $>2,000$ \\
\hline
\end{tabular}


TABLE 2. GEOChEMICAL DATA FOR NONMAGNeTIC, heAVY-MINERAL-CONCENTRATE SAMPLES, LIME hILLS QUADRANGLE, ALASKA--Cont inUEd

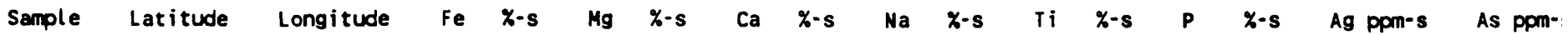

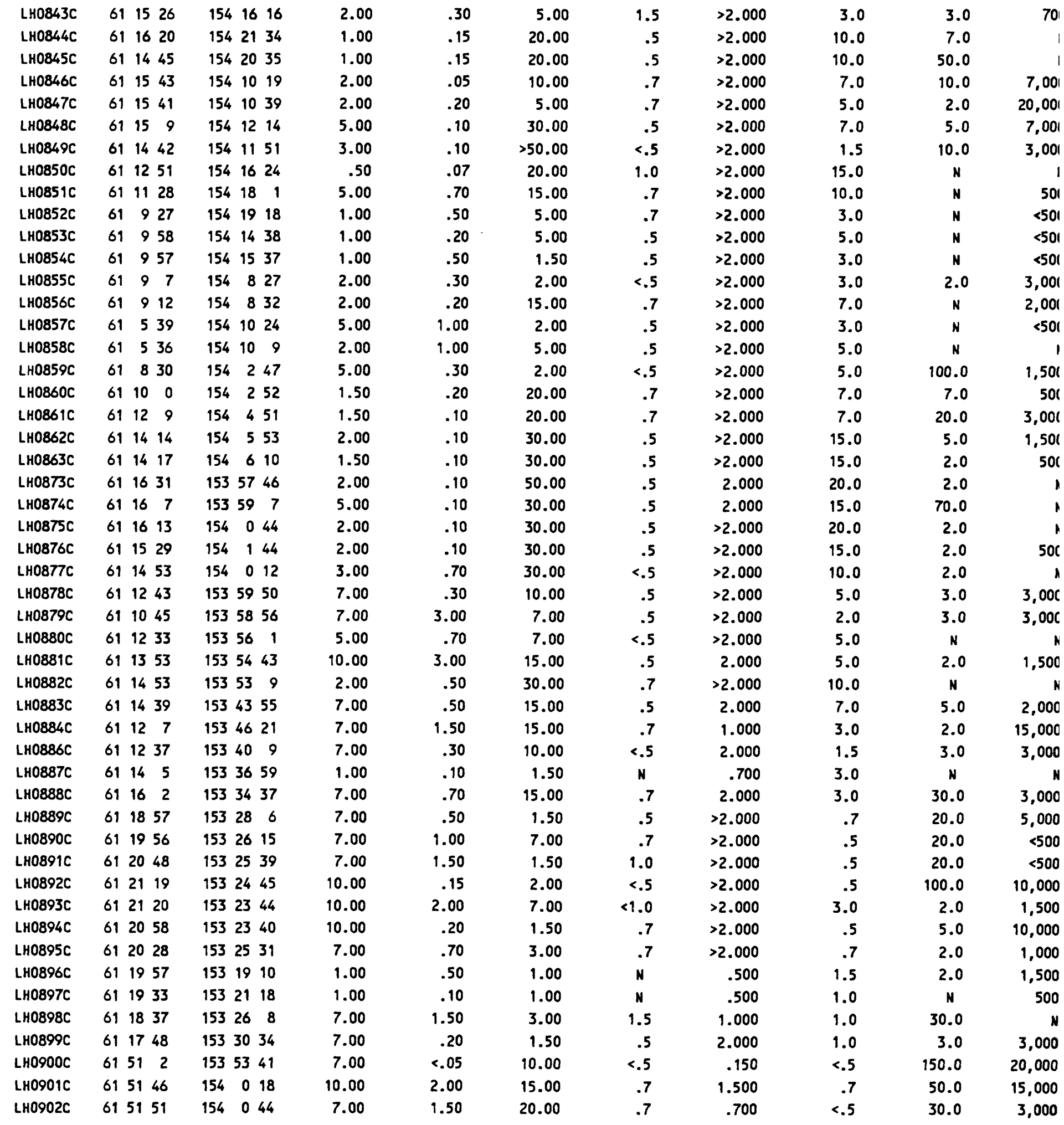




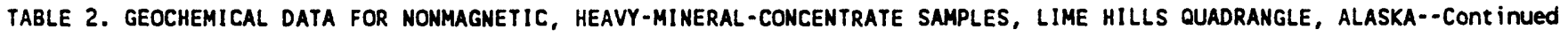

Sample AU ppm-s B ppm-s Ba ppm-s Be ppm-s Bi ppm-s $\quad$ Cd ppm-s Co ppm-s Cr ppm-s Cu ppm-s

\begin{tabular}{|c|c|c|c|c|c|c|c|c|c|}
\hline LHO843C & $N$ & 1,000 & 2,000 & 7 & N & N & 70 & 50 & 15 \\
\hline LH0844C & $N$ & 500 & 500 & 3 & 2,000 & $N$ & $N$ & 50 & 20 \\
\hline LH0845C & $N$ & 150 & 300 & 2 & 300 & $\mathbf{N}$ & N & 50 & 15 \\
\hline LH0846C & $N$ & 150 & 300 & 2 & 100 & $N$ & 300 & $<20$ & 100 \\
\hline LH0847C & $\mathbf{N}$ & 300 & 300 & 5 & 70 & $\mathbf{N}$ & 700 & 70 & 30 \\
\hline LHO848C & $\mathbf{N}$ & $N$ & 100 & 200 & 30 & $\mathbf{N}$ & 100 & 20 & 20 \\
\hline LHO849C & $N$ & $N$ & 70 & 15 & $<20$ & N & 30 & $<20$ & 10 \\
\hline LHO850C & $\mathbf{N}$ & 30 & 300 & 5 & 200 & $\mathbf{N}$ & $<20$ & $<20$ & $<10$ \\
\hline LH0851C & $\mathbf{N}$ & 30 & $>10,000$ & N & $N$ & $\mathbf{N}$ & 50 & 300 & 50 \\
\hline LH0852C & $N$ & 30 & 7,000 & $N$ & $N$ & $N$ & $<20$ & 150 & 20 \\
\hline LH0853C & $\mathbf{N}$ & 50 & $>10,000$ & $N$ & $\mathbf{N}$ & $\mathbf{N}$ & 50 & 200 & 50 \\
\hline LH0854C & $\mathbf{N}$ & 30 & $>10,000$ & 2 & $\mathbf{N}$ & $\mathbf{N}$ & 20 & 200 & 30 \\
\hline LH0855C & $N$ & 500 & $>10,000$ & $N$ & 30 & $\mathbf{N}$ & 20 & 70 & 50 \\
\hline LH0856C & $\mathbf{N}$ & 150 & 7,000 & 2 & 30 & $N$ & 20 & 150 & 150 \\
\hline LH0857C & $N$ & 50 & 5,000 & 2 & $N$ & $N$ & 30 & 200 & 50 \\
\hline LHO858C & N & 50 & $>10,000$ & $\mathbf{N}$ & $N$ & $\mathbf{N}$ & 20 & 200 & 50 \\
\hline LH0859C & 300 & N & $>10,000$ & $N$ & $N$ & $\mathbf{N}$ & 70 & 70 & 500 \\
\hline LH0860C & $N$ & 150 & 1,500 & 3 & 30 & $N$ & 20 & 70 & 100 \\
\hline LH0861C & $N$ & 150 & 300 & 7 & 300 & $\mathbf{N}$ & 50 & $<20$ & 70 \\
\hline LHO862C & $N$ & 30 & 150 & 3 & 30 & N & 70 & 30 & 150 \\
\hline LH0863C & $N$ & 200 & 150 & 7 & $<20$ & $\mathbf{N}$ & $<20$ & 30 & 150 \\
\hline LHO873C & $\mathbf{N}$ & 500 & 300 & 5 & N & N & N & 30 & 30 \\
\hline LHO874C & $N$ & 150 & 200 & 2 & $N$ & N & 30 & 30 & 70 \\
\hline LH0875C & $N$ & 200 & 150 & $N$ & 200 & $N$ & $N$ & $<20$ & 30 \\
\hline LH0876C & N & 200 & 150 & 3 & N & $N$ & 30 & 30 & 70 \\
\hline LH0877C & $N$ & 1,000 & 200 & 2 & 200 & N & $<20$ & 150 & 10 \\
\hline LH0878C & $N$ & 3,000 & 7,000 & 5 & 700 & N & 100 & 70 & 200 \\
\hline LHO879C & $N$ & 200 & 10,000 & 2 & 50 & $\mathbf{N}$ & 70 & 500 & 150 \\
\hline LHO880C & $N$ & 500 & 3,000 & 2 & $\mathbf{N}$ & N & 20 & 70 & 150 \\
\hline LHO881C & $N$ & 2,000 & $>10,000$ & 3 & $\mathbf{N}$ & $\mathbf{N}$ & 100 & 700 & 1,000 \\
\hline LHO882C & $N$ & 500 & 500 & 2 & $\mathbf{N}$ & $N$ & $N$ & 70 & 10 \\
\hline LHO883C & $N$ & 20 & 3,000 & N & $\mathbf{N}$ & $N$ & 100 & 150 & 500 \\
\hline LH0884C & $N$ & 20 & 700 & 3 & $\mathbf{N}$ & $N$ & 200 & 500 & 150 \\
\hline LHO886C & $\mathbf{N}$ & 100 & 500 & 2 & $\mathbf{N}$ & $N$ & 30 & 300 & 100 \\
\hline LH0887C & $N$ & $N$ & 150 & $\mathbf{N}$ & $\mathbf{N}$ & N & $N$ & $<20$ & 70 \\
\hline LH0888C & N & 20 & 700 & 3 & $N$ & $N$ & 50 & 50 & 200 \\
\hline LHO889C & $\mathbf{N}$ & $\mathbf{N}$ & 2,000 & 3 & 20 & $N$ & 20 & 100 & 1,000 \\
\hline LH0890C & $N$ & $N$ & 2,000 & 5 & 30 & $N$ & $<20$ & 200 & 300 \\
\hline LH0891C & $N$ & N & 300 & 5 & 20 & $N$ & $<20$ & 30 & 70 \\
\hline LH0892C & $N$ & N & 150 & 3 & 50 & $\mathbf{N}$ & $<20$ & 150 & 30 \\
\hline LH0893C & $N$ & 1,500 & 700 & 5 & $N$ & $N$ & $<50$ & 150 & 150 \\
\hline LHO894C & $N$ & $N$ & 300 & 3 & 50 & N & 50 & $<20$ & 100 \\
\hline LHO895C & $N$ & $\mathbf{N}$ & 500 & 5 & 50 & N & $<20$ & 30 & 30 \\
\hline LHO896C & N & 100 & 150 & 2 & 500 & $N$ & N & 70 & 70 \\
\hline LH0897C & N & $N$ & 150 & $N$ & 700 & $N$ & N & $<20$ & 50 \\
\hline LH0898C & $N$ & $\mathbf{N}$ & 10,000 & 3 & 500 & 150 & $<20$ & 150 & 100 \\
\hline LH0899C & $N$ & N & 5,000 & 3 & 50 & N & 30 & 50 & 70 \\
\hline LHO9OOC & $N$ & 30 & 300 & 3 & 700 & $N$ & 50 & N & 150 \\
\hline LH0901C & $N$ & 2,000 & $>10,000$ & 2 & 100 & $\mathbf{N}$ & 500 & 150 & 150 \\
\hline LH0902C & $N$ & 1,500 & 1,500 & 2 & $<20$ & $\mathbf{N}$ & 500 & 200 & 50 \\
\hline
\end{tabular}


TABLE 2. GEOCHEMICAL DATA FOR NONMAGNETIC, hEAVY-MINERAL-CONCENTRATE SAMPLES, LIME hILLS QUADRANGLE, ALASKA--ContinUEd

Sample Ga ppm-s Ge ppm-s La ppm-s Mn ppm-s Mo ppm-s Nb ppm-s Ni ppm-s Pb ppm-s Pt ppm-s Sb ppm-s

\begin{tabular}{|c|c|c|c|c|c|c|c|c|c|c|}
\hline LH0843C & 20 & N & 500 & 3,000 & $N$ & 200 & 30 & 300 & N & N \\
\hline LH0844C & 30 & N & 100 & 3,000 & $N$ & 200 & 15 & 20 & N & $N$ \\
\hline LH0845C & 10 & N & 300 & 5,000 & $N$ & 200 & 10 & $<20$ & N & $N$ \\
\hline LH0846C & $<10$ & $N$ & 1,500 & 3,000 & N & 200 & 500 & 100 & N & $N$ \\
\hline LH0847C & 10 & $N$ & 300 & 1,500 & N & 700 & 200 & 30 & N & $N$ \\
\hline LH0848C & 10 & $N$ & 700 & 3,000 & N & 200 & 70 & 100 & N & $N$ \\
\hline LH0849C & $<10$ & $N$ & 700 & 1,500 & N & 150 & 15 & 30 & $N$ & $\mathbf{N}$ \\
\hline LH0850C & $<10$ & $N$ & 500 & 5,000 & N & 100 & 10 & $<20$ & $N$ & N \\
\hline LH0851C & $<10$ & $N$ & 200 & 2,000 & N & 150 & 50 & 50 & $N$ & 7,000 \\
\hline LH0852C & 10 & $N$ & 200 & 700 & N & 100 & 20 & 30 & $N$ & 5,000 \\
\hline LH0853C & $<10$ & $N$ & 700 & 200 & $N$ & 150 & 30 & 50 & $N$ & $N$ \\
\hline LH0854C & $<10$ & N & 700 & 500 & N & 200 & 20 & 30 & $N$ & $N$ \\
\hline LH0855C & $<10$ & N & 500 & 300 & N & 150 & 30 & 30 & $N$ & $\mathbf{N}$ \\
\hline LH0856C & 10 & $N$ & 1,000 & 5,000 & N & 150 & 30 & 20 & $N$ & $\mathbf{N}$ \\
\hline LH0857C & $<10$ & N & 1,000 & 1,500 & N & 150 & 50 & 30 & $N$ & $N$ \\
\hline LH0858C & 10 & N & 300 & 1,500 & $N$ & 100 & 30 & 70 & $N$ & N \\
\hline LH0859C & $<10$ & N & 200 & 2,000 & $N$ & 200 & 100 & 10,000 & N & $<200$ \\
\hline LH0860C & 10 & N & 500 & 5,000 & N & 150 & 10 & 100 & N & N \\
\hline LH0861C & 30 & N & 300 & 5,000 & $N$ & 300 & 30 & 500 & N & N \\
\hline LH0862C & 10 & N & 1,500 & 7,000 & 20 & 200 & 50 & 150 & N & N \\
\hline LH0863C & 10 & $N$ & 700 & 7,000 & $N$ & 500 & 15 & 20 & $N$ & $N$ \\
\hline LH0873C & $<10$ & $N$ & 1,000 & 10,000 & N & 50 & $<10$ & $<20$ & $N$ & $N$ \\
\hline LH0874C & 10 & $N$ & 1,000 & 7,000 & N & 50 & 30 & 1,500 & $N$ & N \\
\hline LH0875C & $<10$ & $N$ & 1,000 & 10,000 & N & 70 & $<10$ & 20 & $N$ & N \\
\hline LH0876C & $<10$ & $N$ & 1,500 & 7,000 & N & 150 & 20 & 20 & $N$ & N \\
\hline LH0877C & 10 & $N$ & $>2,000$ & 7,000 & $N$ & 50 & 10 & 20 & N & $\mathbf{N}$ \\
\hline LH0878C & 30 & $N$ & 700 & 3,000 & N & 100 & 150 & 30 & N & N \\
\hline LH0879C & 10 & N & 500 & 3,000 & N & 70 & 150 & 50 & N & $N$ \\
\hline LHO880C & $<10$ & $N$ & 1,500 & 2,000 & N & 100 & 50 & $<20$ & $N$ & $N$ \\
\hline LHO881C & 20 & N & 700 & 5,000 & N & 50 & 200 & 70 & N & $N$ \\
\hline LH0882C & 10 & N & 1,000 & 7,000 & N & 100 & 10 & $<20$ & $N$ & $N$ \\
\hline LH0883C & 10 & N & 700 & 1,500 & N & $<50$ & 150 & 150 & $N$ & N \\
\hline LHO884C & 30 & $N$ & 200 & 700 & N & $<50$ & 150 & $N$ & $N$ & N \\
\hline LH0886C & 50 & $N$ & 100 & 1,000 & $N$ & $<50$ & 70 & 70 & $N$ & N \\
\hline LH0887C & N & N & 1,000 & 500 & $N$ & $<50$ & $N$ & 50 & $N$ & N \\
\hline LH0888C & 30 & $N$ & 200 & 1,500 & 150 & $<50$ & 30 & 10,000 & $N$ & $N$ \\
\hline LH0889C & 15 & $N$ & 200 & 700 & 30 & 100 & 10 & 1,000 & N & N \\
\hline LH0890С & 50 & N & 200 & 2,000 & 700 & 100 & 10 & 5,000 & N & N \\
\hline LH0891C & 50 & N & 200 & 3,000 & 30 & 50 & $<10$ & 700 & N & N \\
\hline LH0892C & 15 & N & 150 & 1,500 & 300 & 70 & $<10$ & 1,500 & $N$ & N \\
\hline LH0893C & $<20$ & N & 300 & 5,000 & $N$ & 200 & 20 & 100 & $N$ & N \\
\hline LH0894C & 20 & N & 150 & 1,000 & 10 & 70 & $<10$ & 500 & $N$ & $N$ \\
\hline LH0895C & 30 & $N$ & 200 & 2,000 & 100 & 70 & $<10$ & 700 & N & N \\
\hline LH0896C & $N$ & $N$ & $>2,000$ & 3,000 & 30 & 200 & N & 100 & N & N \\
\hline LH0897C & $N$ & N & $>2,000$ & 2,000 & 50 & 150 & $N$ & 150 & N & N \\
\hline LH0898C & 30 & $N$ & 1,500 & 1,500 & 150 & 50 & $<10$ & 1,000 & N & N \\
\hline LH0899C & 10 & N & 1,500 & 1,500 & 50 & 70 & $<10$ & 150 & N & $N$ \\
\hline LH0900C & $<10$ & N & 500 & 200 & 150 & 50 & $N$ & 10,000 & $N$ & $N$ \\
\hline LH0901C & 15 & N & 100 & 500 & $N$ & $<50$ & 200 & 10,000 & $N$ & $<200$ \\
\hline LH0902C & 20 & N & $N$ & 500 & N & $<50$ & 500 & 3,000 & $N$ & 200 \\
\hline
\end{tabular}




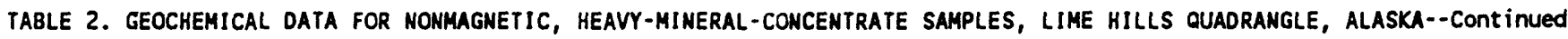
Sample Sc ppm-s Sn ppm-s Sr ppm-s Th ppm-s U ppm-s V ppm-s $\quad W$ ppm-s $\quad Y$ ppm-s Zn ppm-s Zr ppm-s

\begin{tabular}{|c|c|c|c|c|c|c|c|c|c|c|}
\hline LH0843C & 100 & $>2,000$ & $<200$ & N & $<1,000$ & 30 & 200 & 700 & $\mathbf{N}$ & $>2,000$ \\
\hline LH0844C & 100 & $>2,000$ & 700 & $N$ & N & 150 & 700 & 500 & N & $>2,000$ \\
\hline LH0845C & 150 & $>2,000$ & $<200$ & $N$ & N & 150 & 300 & 700 & N & $>2,000$ \\
\hline LH $0846 C$ & 200 & $>2,000$ & $<200$ & $\mathbf{N}$ & $<1,000$ & 30 & 200 & 700 & $N$ & $>2,000$ \\
\hline LH0847C & 200 & $>2,000$ & N & $N$ & $<1,000$ & 150 & 300 & 200 & $\mathbf{N}$ & $>2,000$ \\
\hline LHOB48C & 100 & $>2,000$ & $<200$ & N & N & 30 & 100 & 700 & $N$ & $>2,000$ \\
\hline LH0849C & 50 & 500 & 200 & N & $N$ & 20 & 100 & 700 & N & $>2,000$ \\
\hline LHO850C & 150 & $>2,000$ & $<200$ & $N$ & N & 50 & 700 & 700 & N & $>2,000$ \\
\hline LHO851C & 30 & 700 & 5,000 & $N$ & N & 300 & N & 200 & N & $>2,000$ \\
\hline LHO852C & 50 & 2,000 & 700 & $N$ & N & 200 & 70 & 200 & $N$ & $>2,000$ \\
\hline LH0853C & 20 & 70 & 2,000 & $\mathbf{N}$ & N & 200 & $\mathbf{N}$ & 200 & $N$ & $>2,000$ \\
\hline LHO854C & 150 & 1,000 & 1,500 & N & N & 700 & N & 200 & N & $>2,000$ \\
\hline LHO855C & 100 & $>2,000$ & 1.500 & $\mathbf{N}$ & N & 200 & 10,000 & 150 & $N$ & $>2,000$ \\
\hline LH0856C & 150 & $>2,000$ & 1,000 & $\mathbf{N}$ & N & 200 & 1,000 & 500 & N & $>2,000$ \\
\hline LH0857C & 100 & 500 & 700 & $\mathbf{N}$ & $\mathbf{N}$ & 500 & N & 200 & $N$ & $>2,000$ \\
\hline LH0858C & 50 & 150 & 3,000 & $\mathbf{N}$ & $\mathbf{N}$ & 300 & $N$ & 200 & $\mathbf{N}$ & $>2,000$ \\
\hline LH0859C & 100 & $>2,000$ & 1,000 & N & N & 300 & 70 & 100 & $N$ & $>2,000$ \\
\hline L.H0860C & 70 & $>2,000$ & 300 & N & $\mathbf{N}$ & 70 & 200 & 1,000 & $N$ & $>2,000$ \\
\hline LH08s1C & 30 & $>2,000$ & $<200$ & $N$ & N & 30 & 500 & 700 & N & $>2,000$ \\
\hline LH $0862 \mathrm{C}$ & 150 & 1,000 & $<200$ & $<200$ & $\mathbf{N}$ & 70 & $N$ & 1,000 & 1,000 & $>2,000$ \\
\hline LH0863C & 100 & $>2,000$ & $<200$ & N & $N$ & 50 & 200 & 1,000 & N & $>2,000$ \\
\hline LH0873C & 50 & $>2,000$ & $<200$ & N & N & 30 & N & 2,000 & N & $>2,000$ \\
\hline LH0874C & 70 & 2,000 & $<200$ & $\mathbf{N}$ & 1,000 & 30 & 70 & 1,500 & 5,000 & $>2,000$ \\
\hline LH0875C & 100 & $>2,000$ & $<200$ & $\mathbf{N}$ & 1,000 & 30 & 200 & 1,500 & N & $>2,000$ \\
\hline Lн0876C & 100 & 1,500 & $<200$ & N & N & 50 & 70 & 1,500 & N & $>2,000$ \\
\hline LH0877C & 200 & $>2,000$ & $<200$ & 700 & 1,500 & 50 & 50 & 1,500 & N & $>2,000$ \\
\hline LH0878C & 100 & $>2,000$ & 700 & N & 1,000 & 150 & 200 & 500 & N & $>2,000$ \\
\hline LH0879C & 100 & $>2,000$ & 700 & N & N & 200 & 100 & 200 & N & $>2,000$ \\
\hline LHO880C & 150 & $>2,000$ & 200 & N & N & 150 & 300 & 500 & N & $>2,000$ \\
\hline LH0881C & 70 & $>2,000$ & 1,500 & N & $<1,000$ & 150 & 500 & 300 & 1,000 & $>2,000$ \\
\hline LH0882C & 100 & $>2,000$ & 200 & N & N & 30 & 200 & 1,000 & N & $>2,000$ \\
\hline LH0883C & 70 & 70 & 200 & 700 & 1.500 & 150 & 150 & 500 & $\mathbf{N}$ & $>2,000$ \\
\hline LHO884C & 20 & 30 & 200 & $N$ & N & 150 & N & 200 & $\mathbf{N}$ & $>2,000$ \\
\hline LH0886C & 15 & 20 & $<200$ & N & N & 300 & 200 & 150 & $\mathbf{N}$ & $>2,000$ \\
\hline LH0887C & 100 & $N$ & N & 5,000 & 7,000 & 50 & 700 & 700 & $\mathbf{N}$ & $>2,000$ \\
\hline LH0888C & 30 & N & 200 & N & N & 300 & 150 & 300 & N & $>2,000$ \\
\hline LH0889C & 100 & 100 & $<200$ & $\mathbf{N}$ & N & 70 & $N$ & 500 & 5,000 & $>2,000$ \\
\hline LH0890C & 70 & 300 & 200 & $\mathbf{N}$ & N & 300 & $\mathbf{N}$ & 300 & 2,000 & $>2,000$ \\
\hline LH0891C & 100 & 200 & $<200$ & $N$ & N & 100 & $\mathbf{N}$ & 700 & N & $>2,000$ \\
\hline LH0892C & 150 & 30 & N & $\mathbf{N}$ & N & 50 & N & 700 & N & $>2,000$ \\
\hline LH0893C & 100 & 150 & $\mathbf{N}$ & $N$ & N & 70 & $N$ & 500 & 2,000 & $>5,000$ \\
\hline LH0894C & 70 & 30 & N & N & $\mathbf{N}$ & 50 & N & 500 & N & $>2,000$ \\
\hline LH0895C & 70 & 20 & $<200$ & N & $\mathbf{N}$ & 70 & $\mathbf{N}$ & 500 & $\mathbf{N}$ & $>2,000$ \\
\hline LH0896C & 200 & 700 & $N$ & $>5,000$ & 7,000 & 20 & 200 & $>5,000$ & $\mathbf{N}$ & $>2,000$ \\
\hline LH0897C & 200 & 1,500 & N & $>5,000$ & 10,000 & $<20$ & 200 & $>5,000$ & N & $>2,000$ \\
\hline LH0898C & 150 & 500 & 300 & 300 & N & 150 & 500 & 700 & 10,000 & $>2,000$ \\
\hline LH0899C & 150 & 700 & N & 700 & 1,500 & 70 & 200 & 1,000 & N & $>2,000$ \\
\hline L.H0900C & 70 & 1,500 & $<200$ & $>5,000$ & 7,000 & N & 500 & $>5,000$ & N & $>2,000$ \\
\hline LH0901C & 15 & 50 & 1,500 & N & N & 150 & N & 150 & $N$ & $>2,000$ \\
\hline LH0902C & 15 & $\mathbf{N}$ & 500 & $N$ & $N$ & 300 & 150 & 100 & $\mathbf{N}$ & $>2,000$ \\
\hline
\end{tabular}


TABLE 2. GEOCHEMICAL DATA FOR NONMAGNETIC, heAVy-MINERAL-CONCENTRATE SAMPLES, Lime hills QUADRANGLE, ALASKA--Cont inUed

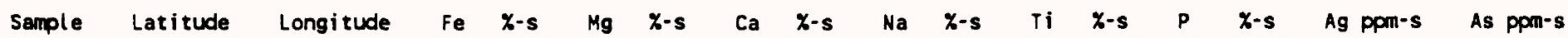

\begin{tabular}{|c|c|c|c|c|c|c|c|c|c|c|c|c|c|}
\hline LH0903C & 6148 & 4 & 153 & 58 & 1 & 30.00 & .07 & 1.00 & $<.5$ & .500 & .7 & 7.0 & 7,000 \\
\hline L.H0904C & 6147 & 41 & 153 & 57 & 3 & 3.00 & .05 & 2.00 & .5 & .700 & .5 & 30.0 & 10,000 \\
\hline LH0905C & 6143 & 37 & 154 & 0 & 13 & 50.00 & .30 & 1.00 & $<.5$ & .700 & $\mathbf{N}$ & 10.0 & 1,500 \\
\hline LH0906C & 6143 & 42 & 153 & 59 & 58 & 5.00 & 1.50 & 10.00 & 1.5 & $>2.000$ & N & $N$ & $N$ \\
\hline LH0907C & 6143 & 38 & 153 & 58 & 57 & 10.00 & .50 & 5.00 & .7 & $>2.000$ & 5.0 & 10.0 & 7,000 \\
\hline LH0908C & 6142 & 41 & 153 & 57 & 0 & 2.00 & $<.05$ & 10.00 & $<.5$ & $>2.000$ & 7.0 & 10.0 & 15,000 \\
\hline LH0909C & 6142 & 41 & 153 & 57 & 0 & 2.00 & .05 & 10.00 & .5 & $>2.000$ & 7.0 & 7.0 & 7,000 \\
\hline LH0910C & 6141 & 37 & 153 & 56 & 59 & 1.00 & .05 & 7.00 & .5 & $>2.000$ & 7.0 & 10.0 & 3,000 \\
\hline LH0911C & 6137 & 28 & 153 & 53 & 33 & 5.00 & .15 & 5.00 & .5 & $>2.000$ & 7.0 & 200.0 & 2,000 \\
\hline LH0912C & 6123 & 7 & 154 & 42 & 29 & 7.00 & .07 & 5.00 & .5 & 2.000 & 5.0 & 20.0 & $>20,000$ \\
\hline LH0913C & 6123 & 43 & 154 & 11 & 5 & 1.00 & $.15^{\circ}$ & 30.00 & $<.5$ & $>2.000$ & .7 & 5.0 & 3,000 \\
\hline LH0914C & 6122 & 19 & 154 & 93 & 33 & 2.00 & .20 & 20.00 & .5 & $>2.000$ & 10.0 & 5.0 & 15,000 \\
\hline LH0915C & 6119 & 32 & 154 & 2 & 44 & 2.00 & .07 & 30.00 & .5 & $>2.000$ & 10.0 & 5.0 & 7,000 \\
\hline LH0916C & 6119 & 24 & 154 & 4 & 19 & 5.00 & .10 & 30.00 & $<.5$ & 2.000 & 15.0 & 5.0 & 7,000 \\
\hline LH0917C & 6119 & 18 & 154 & 5 & 16 & 2.00 & .20 & 20.00 & .5 & $>2.000$ & 3.0 & 2.0 & 3,000 \\
\hline LH0918C & 6118 & 48 & 154 & 73 & 32 & 2.00 & .10 & 2.00 & $<.5$ & $>2.000$ & 7.0 & 20.0 & 15,000 \\
\hline LH0919C & 6120 & 14 & 154 & 9 & 1 & 3.00 & .30 & 20.00 & .7 & $>2.000$ & 1.5 & 5.0 & 15,000 \\
\hline LH0920C & 6124 & 43 & 154 & 123 & 37 & 2.00 & .10 & 30.00 & .7 & $>2.000$ & 5.0 & $N$ & 500 \\
\hline LH0921C & 6127 & 16 & 154 & 53 & 31 & 2.00 & .07 & 30.00 & $<.5$ & $>2.000$ & 7.0 & N & 7,000 \\
\hline LH0922C & 6127 & 46 & 154 & 8 & 7 & 1.00 & .07 & 20.00 & .7 & $>2.000$ & 5.0 & $\mathbf{N}$ & 2,000 \\
\hline LH0923C & 6128 & 40 & 154 & 102 & 29 & 5.00 & .10 & 20.00 & $<.5$ & $>2.000$ & 5.0 & 5.0 & 20,000 \\
\hline LH0924C & 6128 & 3 & 154 & 10 & 40 & 2.00 & .20 & 15.00 & .7 & $>2.000$ & 3.0 & 7.0 & 3,000 \\
\hline LH0925C & 6129 & 1 & 154 & 114 & 43 & 1.00 & .05 & 20.00 & $<.5$ & 2.000 & .7 & 5.0 & 3,000 \\
\hline LH0926C & 6126 & 29 & 154 & 92 & 29 & 3.00 & .05 & 20.00 & $<.5$ & $>2.000$ & 1.0 & 10.0 & $>20,000$ \\
\hline LH0927C & 6127 & 37 & 154 & 132 & 28 & .70 & $<.05$ & 15.00 & $N$ & 2.000 & 1.5 & $N$ & 2,000 \\
\hline LH0929C & 6125 & 33 & 154 & 161 & 15 & 1.00 & .30 & 20.00 & .7 & $>2.000$ & 2.0 & 30.0 & 500 \\
\hline LH0930C & 6124 & 20 & 154 & 174 & 49 & 7.00 & 1.00 & 2.00 & .7 & $>2.000$ & 2.0 & 10.0 & 1,000 \\
\hline LH0931C & 6123 & 58 & 154 & 211 & 11 & 7.00 & 1.50 & 5.00 & .7 & $>2.000$ & 3.0 & $N$ & 1,000 \\
\hline LH0932C & 6122 & 25 & 154 & 224 & 45 & 7.00 & 2.00 & 5.00 & .5 & $>2.000$ & 3.0 & $N$ & 700 \\
\hline LH0933C & 6122 & 34 & 154 & 253 & 35 & 2.00 & .70 & 10.00 & .5 & $>2.000$ & 5.0 & 5.0 & N \\
\hline LH0934C & 6120 & 25 & 154 & 233 & 36 & 2.00 & 1.00 & 2.00 & .5 & $>2.000$ & 2.0 & $\mathbf{N}$ & $N$ \\
\hline LH0935C & 6118 & 7 & 154 & 143 & 30 & 5.00 & .15 & 20.00 & .7 & $>2.000$ & 10.0 & $N$ & 500 \\
\hline LH0936C & 6147 & 11 & 154 & 193 & 30 & 7.00 & 5.00 & 15.00 & .7 & .300 & N & 7.0 & 3,000 \\
\hline LH0937C & 6147 & 30 & 154 & 223 & 34 & 7.00 & .30 & 5.00 & .5 & 2.000 & 1.5 & 7.0 & $<500$ \\
\hline LH0938C & 6146 & 51 & 154 & 252 & 25 & .50 & .15 & 1.50 & .5 & .300 & $\mathbf{N}$ & $\mathbf{N}$ & N \\
\hline LH0939C & 6144 & 19 & 154 & 295 & 55 & 1.00 & 1.00 & 5.00 & 1.0 & 1.500 & 1.0 & $N$ & N \\
\hline LH0940C & 6144 & 29 & 154 & 243 & 30 & 1.50 & .70 & 5.00 & .5 & .700 & 3.0 & $\mathbf{N}$ & N \\
\hline LH0941C & 6116 & 39 & 153 & 575 & 50 & 3.00 & .30 & 30.00 & .5 & 2.000 & 20.0 & $\mathbf{N}$ & N \\
\hline LH0942C & 6116 & 23 & 154 & 02 & 28 & 1.00 & .05 & 20.00 & $<.5$ & $>2.000$ & 10.0 & $N$ & 3,000 \\
\hline LH0943C & 6113 & 9 & 154 & 13 & 35 & 2.00 & .10 & 20.00 & .5 & $>2.000$ & 10.0 & 2.0 & 700 \\
\hline LH0944C & 6117 & 14 & 153 & 405 & 58 & .50 & .15 & 1.50 & $<.5$ & $>2.000$ & 3.0 & $N$ & N \\
\hline LH0945C & 6119 & 22 & 153 & 213 & 30 & 7.00 & .50 & 1.50 & .7 & 1.500 & .7 & 3.0 & 3,000 \\
\hline LH0946C & 6118 & 39 & 153 & 255 & 51 & 15.00 & .10 & 1.50 & .5 & $>2.000$ & .7 & 5.0 & 5,000 \\
\hline LH0947C & 6117 & 20 & 153 & 313 & 34 & 5.00 & .30 & 1.50 & 1.5 & 2.000 & 1.5 & 7.0 & 2,000 \\
\hline LH0948C & 6159 & 34 & 154 & 49 & 9 & 1.50 & .70 & 2.00 & .7 & 1.000 & 1.5 & $N$ & N \\
\hline LH0949C & 6157 & 3 & 154 & 512 & 29 & 7.00 & 5.00 & 10.00 & 1.0 & 2.000 & $<.5$ & $N$ & $N$ \\
\hline LH0950C & 6156 & 37 & 154 & 52 & 9 & 7.00 & 5.00 & 20.00 & 1.0 & 1.500 & $N$ & N & $\mathbf{N}$ \\
\hline LH0951C & 6156 & 41 & 154 & 425 & 53 & .10 & .07 & 1.00 & $<.5$ & .050 & $N$ & $N$ & $\mathbf{N}$ \\
\hline LH0952C & 6156 & 26 & 154 & 431 & 18 & .50 & .07 & .30 & $<.5$ & .007 & $N$ & $\mathbf{N}$ & N \\
\hline LH0953C & 6115 & 39 & 153 & 335 & 56 & 3.00 & 1.50 & 10.00 & 1.0 & 1.000 & 1.5 & $\mathbf{N}$ & 500 \\
\hline
\end{tabular}


TABLE 2. GEOCHEMICAL DATA FOR NONMAGNETIC, hEAVY-MINERAL-CONCENTRATE SAMPLES, LIME hILLS QUADRANGLE, ALASKA--ContinUed

Sample AU ppm-s B ppm-s Ba ppm-s Be ppm-s $\quad$ Bi ppm-s Cd ppm-s Co ppm-s Cr ppm-s CU ppm-s

\begin{tabular}{|c|c|c|c|c|c|c|c|c|c|}
\hline LH0903C & $N$ & 50 & 1,500 & $N$ & $N$ & N & 500 & $<20$ & 70 \\
\hline LH0904C & $N$ & 70 & 700 & 5 & 700 & $N$ & N & N & 70 \\
\hline LH0905C & $N$ & $N$ & 300 & $N$ & 20 & $N$ & 500 & 70 & 700 \\
\hline LH0906C & $N$ & 20 & 200 & N & $N$ & $N$ & 30 & 500 & 70 \\
\hline LH0907C & $N$ & 200 & 1,000 & 3 & 500 & $N$ & 150 & 200 & 200 \\
\hline LHO908C & $N$ & $N$ & 70 & 2 & 50 & $N$ & 500 & $<20$ & 100 \\
\hline LH0909C & $N$ & $N$ & 150 & 5 & 200 & $N$ & 500 & $<20$ & 100 \\
\hline LH0910C & $N$ & 20 & 150 & 20 & 70 & $N$ & 200 & $<20$ & 20 \\
\hline LH0911C & N & 200 & 500 & 5 & 50 & $\mathbf{N}$ & 200 & 30 & 200 \\
\hline LH0912C & $N$ & 200 & 150 & 2 & 500 & $N$ & 3,000 & $<20$ & 200 \\
\hline LH0913C & $N$ & 20 & 150 & 7 & $N$ & N & 300 & 70 & 10 \\
\hline LH0914C & $N$ & 700 & 70 & 5 & 70 & $N$ & 500 & 30 & 20 \\
\hline LH0915C & $N$ & 150 & 70 & 2 & 150 & $N$ & 500 & $<20$ & 150 \\
\hline LH0916C & $N$ & 200 & 70 & 2 & 20 & $N$ & 500 & $<20$ & 500 \\
\hline LH0917C & $N$ & 100 & 150 & 3 & N & $N$ & 300 & 30 & 15 \\
\hline LH0918C & $N$ & 700 & 70 & 5 & 150 & $N$ & 700 & 70 & 150 \\
\hline LH0919C & $\mathbf{N}$ & 700 & 100 & 70 & 200 & $N$ & 300 & 70 & 100 \\
\hline LHO92OC & N & 200 & 100 & 50 & N & $N$ & $N$ & 30 & 20 \\
\hline LH0921C & $N$ & 200 & 50 & 15 & $N$ & $N$ & 200 & $<20$ & 150 \\
\hline LH0922C & $N$ & 150 & 100 & 7 & $N$ & $N$ & 150 & $<20$ & 100 \\
\hline LH0923C & $N$ & 200 & 70 & 50 & 100 & $N$ & 500 & $<20$ & 20 \\
\hline LH0924C & $N$ & 70 & 150 & 7 & $N$ & N & 100 & $<20$ & 70 \\
\hline LH0925C & N & 20 & 70 & 5 & 200 & $N$ & 50 & $<20$ & 20 \\
\hline LH0926C & N & 200 & $<50$ & 70 & 150 & $N$ & 200 & 30 & 10 \\
\hline LH0927C & $\mathbf{N}$ & 50 & 50 & 10 & N & $N$ & N & $<20$ & 10 \\
\hline LH0929C & $N$ & 500 & 70 & 50 & N & N & $N$ & 20 & 10 \\
\hline LH0930C & $N$ & 5,000 & 1,000 & 7 & 1,000 & N & 50 & 200 & 500 \\
\hline LH0931C & $N$ & 3,000 & 1,000 & 3 & 300 & $N$ & 70 & 200 & 100 \\
\hline LH0932C & $N$ & 1,000 & 700 & 3 & $N$ & $N$ & 30 & 300 & 100 \\
\hline LH0933C & $N$ & 700 & 500 & 2 & 300 & $N$ & $<20$ & 200 & 50 \\
\hline LH0934C & $N$ & 100 & 300 & N & $N$ & $N$ & 20 & 200 & 50 \\
\hline LH0935C & $N$ & 100 & 100 & 5 & $N$ & N & 20 & 70 & 50 \\
\hline LH0936C & $N$ & 50 & $>10,000$ & 7 & $<20$ & 100 & 70 & 150 & 100 \\
\hline LH0937C & $N$ & 50 & $>10,000$ & $N$ & $N$ & $N$ & 50 & 70 & 700 \\
\hline LH0938C & $N$ & $N$ & $>10,000$ & $N$ & $N$ & $N$ & $N$ & $<20$ & 10 \\
\hline LHO939C & $N$ & 200 & 5,000 & $N$ & N & N & $N$ & 150 & 15 \\
\hline LHO94OC & $\mathbf{N}$ & 150 & $>10,000$ & $N$ & $N$ & $N$ & $<20$ & 150 & 15 \\
\hline LH0941C & $N$ & 3,000 & 70 & 2 & $N$ & $N$ & 20 & 70 & 50 \\
\hline LH0942C & $N$ & 50 & 70 & $N$ & 20 & $N$ & 150 & 20 & 15 \\
\hline LH0943C & $N$ & 200 & 100 & 2 & 20 & $N$ & 100 & 20 & 30 \\
\hline LHO944C & $\mathbf{N}$ & $N$ & 200 & $N$ & $N$ & $N$ & $N$ & $<20$ & 10 \\
\hline LH0945C & $N$ & $N$ & 700 & $N$ & 1,000 & $N$ & 30 & 70 & 50 \\
\hline LH0946C & N & N & 1,500 & N & 300 & $N$ & 100 & $<20$ & 100 \\
\hline LH0947C & N & $N$ & 10,000 & 3 & $N$ & N & 30 & 20 & 20 \\
\hline LH0948C & N & 70 & $>10,000$ & 3 & N & $N$ & $\mathbf{N}$ & 100 & $<10$ \\
\hline LH0949C & N & 150 & 3,000 & 2 & $N$ & $N$ & 50 & 1,000 & 70 \\
\hline LH0950C & N & 200 & 1,000 & $N$ & $N$ & $N$ & 30 & 1,500 & 50 \\
\hline LH0951C & N & $N$ & $>10,000$ & $N$ & $\mathbf{N}$ & $N$ & $N$ & $<20$ & $<10$ \\
\hline LHO952C & $\mathbf{N}$ & $N$ & $>10,000$ & N & $N$ & N & $N$ & $<20$ & $<10$ \\
\hline LH0953C & $N$ & $N$ & $>10,000$ & $N$ & N & N & 70 & 150 & 100 \\
\hline
\end{tabular}




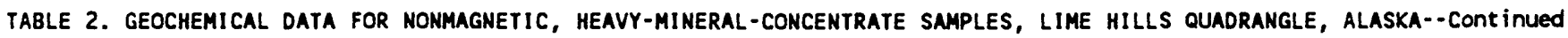

Sample Ga ppm-s Ge ppm-s La ppm-s Mn ppm-s Mo ppm-s Nb ppm-s Ni ppm-s Pb ppm-s Pt ppm-s Sb ppm-s

\begin{tabular}{|c|c|c|c|c|c|c|c|c|}
\hline LH0903C & $<10$ & $\mathbf{N}$ & 300 & 200 & 50 & $<50$ & 700 & 700 \\
\hline LH0904C & $<10$ & N & 700 & 300 & 700 & 50 & $<10$ & 2,000 \\
\hline LH0905C & $<10$ & $\mathbf{N}$ & $N$ & 200 & N & $<50$ & 700 & 2,000 \\
\hline LH0906C & 20 & N & N & 700 & $N$ & $<50$ & 70 & 100 \\
\hline LH0907C & 15 & $N$ & 1,000 & 1,000 & 10 & 70 & 150 & 2,000 \\
\hline LH0908C & $<10$ & N & 1,500 & 1,500 & $\mathbf{N}$ & 50 & 300 & 300 \\
\hline LHO909C & $<10$ & $N$ & 1,500 & 1,500 & N & 70 & 300 & 100 \\
\hline LH0910C & $<10$ & $N$ & 1,000 & 1,500 & N & 100 & 100 & 150 \\
\hline LH0911C & 30 & N & 1,500 & 2,000 & $\mathbf{N}$ & 200 & 50 & 1,000 \\
\hline LH0912C & $<10$ & N & 1,500 & 3,000 & $N$ & 70 & 1,500 & 700 \\
\hline LHO913C & 10 & N & 2,000 & 500 & $N$ & 200 & 100 & 500 \\
\hline LH0914C & 10 & N & $>2,000$ & 7,000 & N & 200 & 300 & 150 \\
\hline LH0915C & $<10$ & N & 2,000 & 7,000 & $N$ & 50 & 300 & 70 \\
\hline LH0916C & $<10$ & N & 1,500 & 7,000 & $N$ & 70 & 500 & 150 \\
\hline LH0917C & 15 & N & 700 & 3,000 & N & 150 & 200 & 100 \\
\hline LH0918C & 15 & N & $>2,000$ & 2,000 & $N$ & 150 & 1,500 & 500 \\
\hline LH0919C & 30 & N & 2,000 & 1,500 & $N$ & 300 & 200 & 100 \\
\hline LH092OC & 70 & N & $>2,000$ & 3,000 & $N$ & 700 & 10 & 100 \\
\hline LH0921C & 10 & N & $>2,000$ & 3,000 & $\mathbf{N}$ & 100 & 300 & 70 \\
\hline LH0922C & 10 & N & $>2,000$ & 2,000 & N & 150 & 100 & 150 \\
\hline LH0923C & $<10$ & $N$ & $>2,000$ & 2,000 & N & 150 & 700 & 150 \\
\hline LH0924C & 20 & $N$ & 2,000 & 1,000 & 200 & 100 & 70 & 2,000 \\
\hline LH0925C & $<10$ & $N$ & $>2,000$ & 700 & N & 150 & 30 & 300 \\
\hline LH0926C & 10 & N & $>2,000$ & 500 & $N$ & 500 & 70 & 1,000 \\
\hline LH0927C & $<10$ & $\mathbf{N}$ & 1,500 & 500 & N & 70 & 10 & 30 \\
\hline LHO929C & 30 & N & 2,000 & 1,500 & $N$ & 300 & $<10$ & 70 \\
\hline LH0930C & 20 & $N$ & 1,500 & 1,000 & 15 & 100 & 150 & 100 \\
\hline LH0931C & 30 & $N$ & 300 & 1,500 & N & 100 & 100 & 100 \\
\hline LH0932C & 15 & N & 500 & 1,500 & N & 150 & 100 & 20 \\
\hline LH0933C & 10 & $\mathbf{N}$ & 500 & 1,500 & $N$ & 150 & 70 & 30 \\
\hline LH0934C & 10 & $N$ & 300 & 700 & N & 150 & 30 & 30 \\
\hline LH0935C & 15 & N & 700 & 5,000 & N & 200 & 20 & 30 \\
\hline LH0936C & 10 & $N$ & N & 500 & N & $<50$ & 50 & 1,000 \\
\hline LH0937C & $<10$ & $N$ & 100 & 100 & $N$ & $<50$ & 100 & 2,000 \\
\hline LH0938C & N & $N$ & N & 50 & N & $<50$ & $<10$ & 30 \\
\hline LH0939C & 15 & $N$ & N & 300 & $N$ & $<50$ & 15 & N \\
\hline LH094OC & 10 & $N$ & 150 & 300 & N & $<50$ & 20 & N \\
\hline LH0941C & 10 & N & 700 & 10,000 & $\mathbf{N}$ & 50 & 15 & 20 \\
\hline LH0942C & $N$ & N & 1,500 & 7,000 & N & 70 & 100 & 70 \\
\hline LH0943C & 10 & N & 700 & 7,000 & N & 100 & 50 & 150 \\
\hline LH0944C & N & N & 700 & 500 & N & 100 & N & 100 \\
\hline LH0945C & 10 & $N$ & 500 & 2,000 & 300 & 50 & $<10$ & 300 \\
\hline LH0946C & $\mathbf{N}$ & N & 200 & 500 & N & 70 & $<10$ & 200 \\
\hline Lн0947C & 30 & $N$ & 1,000 & 700 & 150 & 50 & 10 & 100 \\
\hline LH0948C & $<10$ & $N$ & $<100$ & 200 & 10 & $<50$ & 10 & $N$ \\
\hline LHO949C & 15 & $N$ & $N$ & 1,000 & N & $<50$ & 150 & 20 \\
\hline LH0950С & 15 & N & $N$ & 1,500 & N & $<50$ & 100 & 20 \\
\hline LH0951C & $N$ & N & N & 50 & N & $<50$ & $<10$ & $\mathbf{N}$ \\
\hline LH0952C & N & N & N & 150 & $N$ & N & $<10$ & N \\
\hline LH0953C & 20 & $\mathbf{N}$ & 300 & 1,500 & 100 & $<50$ & 50 & 300 \\
\hline
\end{tabular}


TABLE 2. GEOCHEMICAL DATA FOR NONMAGNETIC, HEAVY-MINERAL-CONCENTRATE SAMPLES, LIME hILLS QUADRANGLE, ALASKA--ContinUed Sample Sc ppm-s Sn ppm-s

Sr ppm-s Th ppm-s

ppm-s

$\checkmark$ ppm-s

$\omega$ ppm-s

Y ppm-s

Zn ppm-s $2 r$ ppm-s

\begin{tabular}{|c|c|c|c|c|c|c|c|c|c|c|}
\hline LH0903C & 30 & 100 & N & 500 & 1,000 & 100 & 200 & 700 & N & $>2,000$ \\
\hline LH0904C & 30 & 700 & N & $>5,000$ & 7,000 & $<20$ & 3,000 & 1,000 & 2,000 & 1,500 \\
\hline LH0905C & $\mathbf{N}$ & N & N & N & N & 100 & N & 20 & 700 & 300 \\
\hline LH0906C & 15 & $\mathbf{N}$ & 500 & N & N & 700 & N & 30 & N & $>2,000$ \\
\hline LH0907C & 70 & 70 & 500 & $<200$ & N & 300 & 10,000 & 500 & N & $>2,000$ \\
\hline LHO908C & 150 & 500 & N & 300 & 2,000 & 20 & 200 & 1,000 & $\mathbf{N}$ & $>2,000$ \\
\hline LH0909C & 150 & 500 & N & 300 & 2,000 & 30 & 200 & 1,000 & N & $>2,000$ \\
\hline LH0910C & 100 & $>2,000$ & $<200$ & 200 & 2,000 & 50 & 200 & 700 & $N$ & $>2,000$ \\
\hline LH0911C & 150 & 1,500 & $<200$ & $<200$ & $\mathbf{N}$ & 100 & 2,000 & 700 & $N$ & $>2,000$ \\
\hline LH0912C & 150 & $>2,000$ & $<200$ & 300 & 7,000 & 30 & 100 & 700 & $N$ & $>2,000$ \\
\hline LH0913C & 150 & 700 & N & $>5,000$ & 3,000 & 70 & 200 & 1,500 & N & $>2,000$ \\
\hline LH0914C & 150 & $>2,000$ & $<200$ & 700 & 3,000 & 30 & 100 & 1,000 & $\mathbf{N}$ & $>2,000$ \\
\hline LH0915C & 150 & $>2,000$ & $<200$ & 300 & 1,500 & 30 & 100 & 1,000 & N & $>2,000$ \\
\hline LH0916C & 100 & 1,500 & $<200$ & 200 & 3,000 & 30 & 150 & 1,500 & $\mathbf{N}$ & $>2,000$ \\
\hline LH0917C & 70 & $>2,000$ & $<200$ & 300 & 10,000 & 50 & 50 & 700 & N & $>2,000$ \\
\hline LH0918C & 150 & $>2,000$ & N & 2,000 & 3,000 & 30 & 200 & 2,000 & 2,000 & $>2,000$ \\
\hline LH0919C & 150 & $>2,000$ & $<200$ & 300 & 3,000 & 50 & 300 & $>5,000$ & N & $>2,000$ \\
\hline LH0920C & 200 & 500 & $<200$ & 3,000 & 1,500 & 70 & 100 & $>5,000$ & N & $>2,000$ \\
\hline LH0921C & .200 & 1,000 & $N$ & 3,000 & 3,000 & 30 & 200 & 3,000 & $\mathbf{N}$ & $>2,000$ \\
\hline LH0922C & 150 & 500 & N & 1,500 & 1,000 & 30 & 150 & 1,500 & $\mathbf{N}$ & $>2,000$ \\
\hline LH0923C & 200 & 1,000 & $<200$ & 5,000 & 7,000 & 70 & 700 & 2,000 & $\mathbf{N}$ & $>2,000$ \\
\hline LH0924C & 70 & 1,500 & $<200$ & $>5,000$ & 5,000 & 100 & 2,000 & 700 & $N$ & $>2,000$ \\
\hline LH0925C & 100 & 100 & N & $>5,000$ & 5,000 & 50 & 500 & $>5,000$ & N & $>2,000$ \\
\hline LH0926C & 200 & 700 & $<200$ & $>5,000$ & 5,000 & 30 & 100 & $>5,000$ & N & $>2,000$ \\
\hline LH0927C & 100 & 700 & N & 3,000 & 2,000 & 50 & 500 & $>5,000$ & N & $>2,000$ \\
\hline LH0929C & 200 & 500 & $<200$ & 1,500 & $<1,000$ & 50 & 50 & $>5,000$ & N & $>2,000$ \\
\hline LH0930C & 100 & $>2,000$ & 200 & N & N & 300 & 300 & 300 & 700 & $>2,000$ \\
\hline LH0931C & 70 & 1,000 & 300 & N & N & 300 & N & 300 & N & $>2,000$ \\
\hline LH0932C & 100 & $>2,000$ & 300 & $\mathbf{N}$ & N & 200 & 500 & 200 & $\mathbf{N}$ & $>2,000$ \\
\hline LH0933C & 100 & $>2,000$ & 300 & $\mathbf{N}$ & N & 200 & 150 & 300 & $N$ & $>2,000$ \\
\hline LH0934C & 200 & $>2,000$ & 200 & N & N & 300 & 100 & 200 & N & $>2,000$ \\
\hline LH0935C & 150 & $>2,000$ & $<200$ & $\mathbf{N}$ & N & 70 & 100 & 1,500 & N & $>2,000$ \\
\hline LH0936C & 10 & 1,000 & 2,000 & $N$ & $\mathbf{N}$ & 100 & 500 & 30 & 7,000 & 700 \\
\hline LH0937C & 15 & 20 & $>10,000$ & $\mathbf{N}$ & $\mathbf{N}$ & 70 & 50 & 100 & N & $>2,000$ \\
\hline LH0938C & $\mathbf{N}$ & N & 7,000 & N & N & 20 & N & 30 & $N$ & $>2,000$ \\
\hline LHO939C & 20 & 700 & 200 & N & N & 150 & 100 & 300 & N & $>2,000$ \\
\hline LHO94OC & 30 & 50 & 300 & 300 & N & 150 & 50 & 300 & N & $>2,000$ \\
\hline LH0941C & 100 & 700 & $<200$ & N & N & 70 & N & 1,500 & $N$ & $>2,000$ \\
\hline LH0942C & 150 & $>2,000$ & $<200$ & 200 & 3,000 & 30 & 300 & 1,000 & N & $>2,000$ \\
\hline LH0943C & 100 & $>2,000$ & $<200$ & N & 3,000 & 50 & 150 & 700 & $\mathbf{N}$ & $>2,000$ \\
\hline LHO944C & 150 & 50 & $<200$ & $>5,000$ & 7,000 & 70 & 100 & 500 & N & $>2,000$ \\
\hline LH0945C & 70 & 700 & $<200$ & 1,000 & 1,500 & 50 & 700 & 700 & 1,000 & $>2,000$ \\
\hline LH0946C & 50 & 200 & N & N & N & 70 & 200 & 500 & N & $>2,000$ \\
\hline LH0947C & 20 & 20 & $<200$ & 5,000 & 3,000 & 70 & 1,000 & 300 & $N$ & $>2,000$ \\
\hline LHO948C & 15 & 1,500 & 1.500 & N & N & 100 & 200 & 300 & $\mathbf{N}$ & $>2,000$ \\
\hline LH0949C & 30 & N & 500 & $\mathbf{N}$ & $\mathbf{N}$ & 150 & N & 30 & N & 2,000 \\
\hline LH0950C & 30 & N & 700 & $\mathbf{N}$ & $N$ & 200 & $\mathbf{N}$ & 30 & N & 1,500 \\
\hline LH0951C & N & 700 & 3,000 & N & $\mathbf{N}$ & N & 70 & 70 & $\mathbf{N}$ & $>2,000$ \\
\hline LH0952C & N & 50 & 5,000 & N & N & N & $N$ & N & 500 & 150 \\
\hline LH0953C & 15 & 20 & 200 & 1,000 & $<1,000$ & 50 & 500 & 200 & N & $>2,000$ \\
\hline
\end{tabular}


TABLE 2. GEOCHEMICAL DATA fOR NONMAGNETIC, hEAVY-MINERAL-CONCENTRATE SAMPLES, LIME hILLS QUAdRANGLE, ALASKA--ContinUed

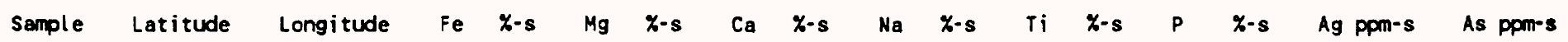

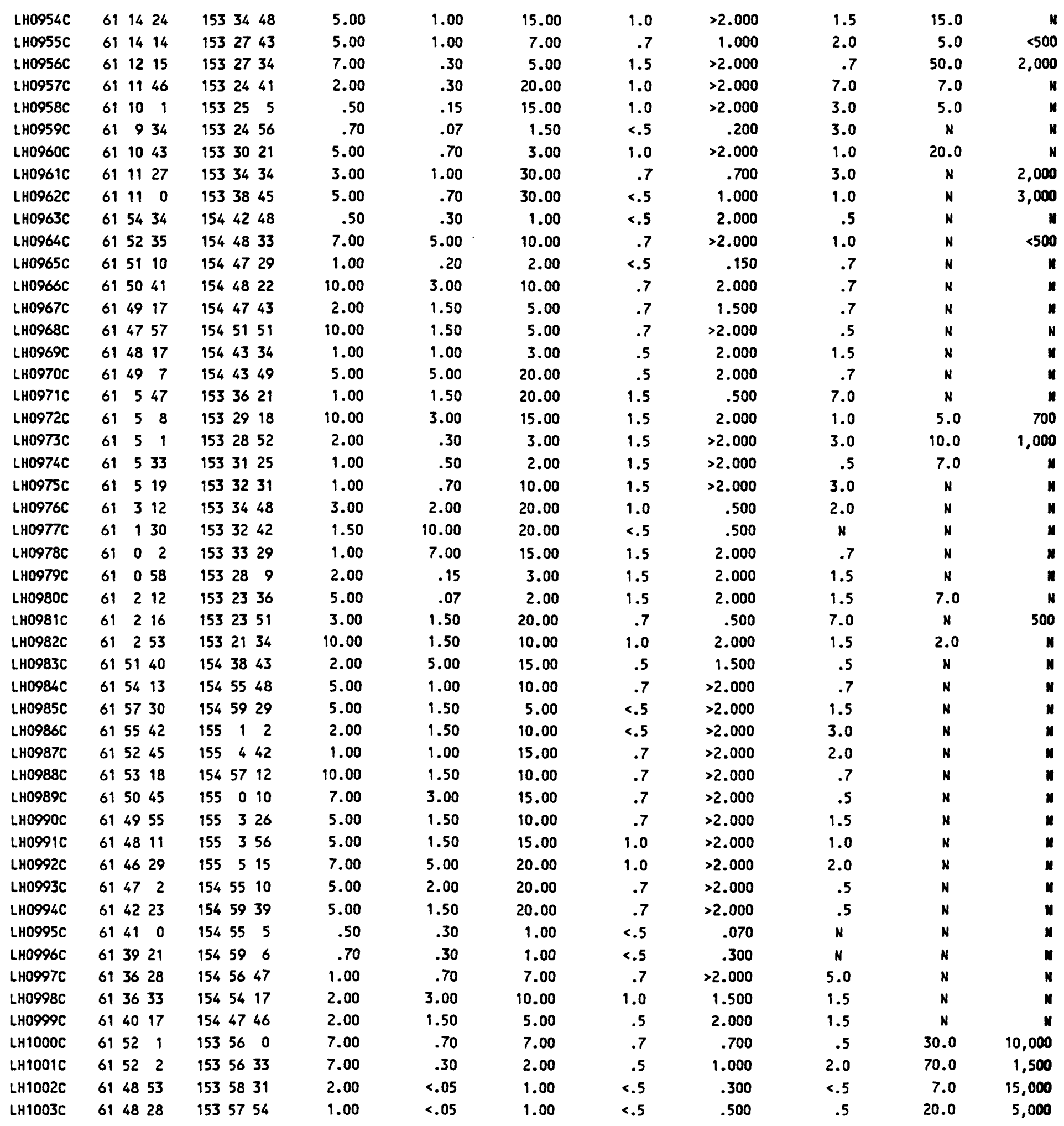


TABLE 2. GEOCHEMICAL DATA FOR NONMAGNETIC, hEAVY-MINERAL-CONCENTRATE SAMPLES, LIME hILLS QUADRANGLE, ALASKA--CONT inUed Sample AU ppm-s B ppm-s Ba ppm-s Be ppm-s $\quad$ Bi ppm-s Cd ppm-s Co ppm-s Cr ppm-s CU ppm-s

\begin{tabular}{|c|c|c|c|c|c|c|c|c|c|}
\hline LH0954C & $N$ & 30 & $>10,000$ & 3 & N & $N$ & 20 & 70 & 70 \\
\hline LH0955C & $N$ & $N$ & 200 & N & 2,000 & $N$ & 30 & 70 & 70 \\
\hline LH0956C & $N$ & 20 & 3,000 & 3 & 100 & N & 100 & $<20$ & 70 \\
\hline LH0957C & $N$ & $N$ & 200 & $N$ & 150 & $N$ & 30 & 20 & 30 \\
\hline LH0958C & $N$ & $N$ & 300 & $\mathbf{N}$ & 150 & N & $<20$ & $<20$ & 20 \\
\hline LHO959C & $N$ & $N$ & 1,500 & $\mathbf{N}$ & $N$ & $N$ & N & $<20$ & $<10$ \\
\hline LH0960C & $N$ & $N$ & $>10,000$ & 3 & $N$ & N & 20 & 30 & 1,000 \\
\hline LH0961C & $N$ & 50 & 300 & 3 & N & N & 70 & 70 & 150 \\
\hline LH0962C & $N$ & 20 & 150 & 5 & $N$ & $N$ & 70 & 70 & 30 \\
\hline LH0963C & $N$ & 20 & $>10,000$ & N & $N$ & $N$ & $N$ & 30 & $<10$ \\
\hline LH0964C & $N$ & 100 & 7,000 & 2 & $N$ & N & 50 & 1,500 & 50 \\
\hline LH0965C & $N$ & 20 & $>10,000$ & $N$ & $N$ & $N$ & $\mathbf{N}$ & $<20$ & 15 \\
\hline LHO966C & $N$ & 2,000 & 10,000 & 30 & $N$ & $N$ & 50 & 500 & 70 \\
\hline LH0967C & $N$ & 700 & $>10,000$ & N & $N$ & N & 20 & 150 & 10 \\
\hline LH0968C & N & 1,000 & $>10,000$ & 3 & $N$ & N & 70 & 100 & 70 \\
\hline LH0969C & $N$ & 500 & 1,500 & 5 & $N$ & N & N & 200 & $<10$ \\
\hline LH0970C & $N$ & 700 & 3,000 & 5 & $N$ & $N$ & $<20$ & 150 & 10 \\
\hline LH0971C & $N$ & N & 1,000 & $N$ & $N$ & $N$ & $<20$ & $<20$ & $<10$ \\
\hline LH0972C & $N$ & 20 & 7,000 & 2 & $<20$ & $N$ & 30 & 300 & 150 \\
\hline LH0973C & $N$ & N & 5,000 & 2 & 150 & $N$ & 20 & $<20$ & 100 \\
\hline LH0974C & $N$ & N & 500 & 3 & N & N & $N$ & $<20$ & $<10$ \\
\hline LH0975C & $N$ & N & 700 & N & $N$ & N & $N$ & 50 & 10 \\
\hline LH0976C & $N$ & 20 & 200 & $N$ & $N$ & $N$ & 100 & 150 & 70 \\
\hline LH0977C & $N$ & 20 & 100 & $N$ & N & $N$ & 20 & $<20$ & 20 \\
\hline LH0978C & $N$ & $N$ & 200 & N & $<20$ & $N$ & $<20$ & $<20$ & 15 \\
\hline LH0979C & $N$ & $N$ & 700 & N & 30 & $N$ & 30 & $<20$ & 70 \\
\hline LH0980C & $N$ & $\mathbf{N}$ & 3,000 & N & 100 & $N$ & 100 & $<20$ & 70 \\
\hline LH0981C & $N$ & 50 & 1,500 & $N$ & $N$ & $N$ & 50 & 70 & 50 \\
\hline LH0982C & $N$ & 1,000 & 2,000 & N & N & N & 70 & 200 & 100 \\
\hline LH0983C & N & 3,000 & 1,000 & 5 & N & $N$ & $<20$ & 100 & 10 \\
\hline LH0984C & $N$ & 500 & 7,000 & 3 & $N$ & N & 30 & 500 & 50 \\
\hline LH0985C & $N$ & 100 & $>10,000$ & 5 & $N$ & $N$ & 70 & 500 & 50 \\
\hline LH0986C & $N$ & 5,000 & $>10,000$ & 5 & $N$ & $N$ & 30 & 500 & 50 \\
\hline LH0987C & N & $>5,000$ & $>10,000$ & 30 & $N$ & $N$ & 20 & 200 & 50 \\
\hline LH0988C & $N$ & 200 & $>10,000$ & $N$ & $N$ & N & 30 & 150 & 70 \\
\hline LH0989C & N & 100 & 1,000 & $N$ & $N$ & N & 30 & 1,000 & 70 \\
\hline LHO990C & $N$ & 500 & $>10,000$ & $N$ & $N$ & $N$ & 30 & 300 & 50 \\
\hline LH0991C & N & 70 & $>10,000$ & $N$ & $\mathbf{N}$ & N & 20 & 300 & 70 \\
\hline LH0992C & N & 70 & 5,000 & $N$ & N & N & 30 & 1,000 & 50 \\
\hline LH0993C & $N$ & 500 & 1,000 & 3 & $\mathbf{N}$ & $N$ & 30 & 300 & 50 \\
\hline LH0994C & N & 700 & 3,000 & 3 & $N$ & $N$ & 30 & 200 & 30 \\
\hline LH0995C & $N$ & $N$ & $>10,000$ & $N$ & $N$ & $N$ & $N$ & 20 & $<10$ \\
\hline LHO996C & $N$ & 20 & $>10,000$ & N & $N$ & N & N & 30 & $<10$ \\
\hline LH0997C & $N$ & 150 & $>10,000$ & 20 & $N$ & N & N & 70 & 10 \\
\hline LH0998С & $N$ & 150 & $>10,000$ & 7 & N & $N$ & $<20$ & 150 & 10 \\
\hline LHO999C & N & 200 & 5,000 & 10 & N & $N$ & $<20$ & 150 & 10 \\
\hline LH1000C & $N$ & 100 & 300 & 7 & 200 & $N$ & 150 & 150 & 70 \\
\hline LH1001C & N & 150 & 300 & $<2$ & $N$ & N & 150 & 70 & 50 \\
\hline LH1002C & $N$ & $N$ & 700 & 3 & 150 & $N$ & 70 & $<20$ & 70 \\
\hline LH1003C & $N$ & $N$ & 200 & 3 & 200 & $N$ & 30 & $<20$ & 50 \\
\hline
\end{tabular}


TABLE 2. GEOCHEMICAL DATA FOR NONMAGNETIC, hEAVY-MINERAL-CONCENTRATE SAMPLES, LIME hILLS QUADRANGLE, ALASKA--CONTINUEd

Sample Ga ppm-s Ge ppm-s La ppm-s Mn ppm-s Mo ppm-s Nb ppm-s Ni ppm-s Pb ppm-s Pt ppm-s Sb ppm-s

\begin{tabular}{|c|c|c|c|c|c|c|c|c|}
\hline LH0954C & 20 & N & 150 & 1,500 & 200 & 70 & 10 & 1,000 \\
\hline LH0955C & 10 & N & 300 & 1,500 & 100 & 50 & 20 & 150 \\
\hline LH0956C & 20 & $N$ & 200 & 1,000 & 500 & 150 & 15 & 500 \\
\hline LH0957C & $<10$ & N & 300 & 1,000 & 100 & $<50$ & $<10$ & 200 \\
\hline LH0958C & $<10$ & $N$ & 150 & 500 & 10 & $<50$ & $<10$ & 30 \\
\hline LH0959C & N & $\mathbf{N}$ & 1,500 & 300 & 100 & $<50$ & $N$ & 70 \\
\hline LH0960C & 20 & $N$ & 700 & 1,000 & 100 & 200 & 10 & 30,000 \\
\hline LH0961C & 30 & N & 100 & 1,500 & $N$ & $<50$ & 50 & 70 \\
\hline LH0962C & 50 & $N$ & 100 & 1,500 & $N$ & $<50$ & 50 & $<20$ \\
\hline LH0963C & N & $N$ & $N$ & 100 & N & $<50$ & $<10$ & $\mathbf{N}$ \\
\hline LH0964C & 10 & $N$ & 150 & 1,500 & $N$ & 50 & 150 & 20 \\
\hline LH0965C & $N$ & N & $N$ & 100 & $N$ & $<50$ & 15 & $N$ \\
\hline LH0966C & 15 & N & 300 & 1,000 & $N$ & 50 & 100 & 30 \\
\hline LH0967C & $<10$ & N & N & 500 & N & $<50$ & 20 & N \\
\hline LH0968C & 20 & $N$ & 100 & 500 & N & 50 & 70 & 50 \\
\hline LH0969C & $<10$ & N & 200 & 300 & $<10$ & 50 & $<10$ & $\mathbf{N}$ \\
\hline LH0970C & 15 & $N$ & 150 & 1,500 & $\mathbf{N}$ & 70 & 20 & N \\
\hline LH0971C & 10 & $N$ & 300 & 500 & $\mathbf{N}$ & $<50$ & $<10$ & N \\
\hline LH0972C & 20 & $N$ & 150 & 3,000 & 15 & 70 & 50 & 3,000 \\
\hline LH0973C & 15 & $N$ & 150 & 700 & $<10$ & $<50$ & $<10$ & 200 \\
\hline LH0974C & 10 & $N$ & 1,500 & 1,000 & 30 & 150 & $<10$ & 300 \\
\hline LH0975C & 10 & N & 150 & 700 & 10 & 70 & 10 & 30 \\
\hline LH0976C & 15 & $N$ & N & 500 & $N$ & $<50$ & 15 & $N$ \\
\hline LH0977C & $<10$ & N & $N$ & 700 & $N$ & $<50$ & 10 & 20 \\
\hline LH0978C & $<10$ & N & 100 & 500 & 10 & 70 & $<10$ & 50 \\
\hline Lн0979C & $<10$ & N & 150 & 300 & $\mathbf{N}$ & 70 & $<10$ & 20 \\
\hline LH0980C & $<10$ & N & 150 & 200 & $N$ & 70 & 10 & 20 \\
\hline LH0981C & $<10$ & $N$ & 100 & 1,500 & N & $<50$ & 30 & 30 \\
\hline LH0982C & 10 & $N$ & N & 1,000 & $N$ & $<50$ & 70 & 700 \\
\hline LH0983C & 10 & $N$ & 300 & 700 & $N$ & 70 & 20 & $<20$ \\
\hline LH0984C & 30 & $N$ & 100 & 700 & $N$ & 100 & 30 & 30 \\
\hline LH0985C & 10 & $N$ & 700 & 500 & $N$ & 300 & 70 & $<20$ \\
\hline LH0986C & $<10$ & $N$ & 200 & 300 & $N$ & 200 & 50 & $<20$ \\
\hline LH0987C & $<10$ & $N$ & 150 & 200 & $\mathbf{N}$ & 200 & 30 & $N$ \\
\hline LH0988C & 15 & N & N & 500 & 10 & 50 & 50 & 20 \\
\hline LH0989C & 15 & N & $<100$ & 700 & $\mathbf{N}$ & 50 & 50 & 150 \\
\hline LH0990C & 15 & N & 100 & 500 & N & 70 & 30 & 20 \\
\hline LH0991C & 15 & N & 200 & 700 & $N$ & 50 & 50 & 20 \\
\hline LH0992C & 30 & N & 100 & 2,000 & N & 50 & 70 & 20 \\
\hline LH0993C & 20 & N & $N$ & 1,000 & N & 50 & 30 & 20 \\
\hline LH0994C & 20 & $N$ & $N$ & 700 & N & 50 & 30 & 20 \\
\hline LH0995C & N & N & $N$ & 70 & $N$ & $<50$ & $<10$ & N \\
\hline LH0996C & N & N & $N$ & 100 & $N$ & $<50$ & $<10$ & $N$ \\
\hline LH0997C & 10 & $N$ & 500 & 500 & N & 70 & $<10$ & $<20$ \\
\hline LH0998C & 15 & $N$ & 100 & 700 & 70 & 50 & 20 & $<20$ \\
\hline LH0999C & 10 & $N$ & 300 & 700 & 30 & 50 & 15 & $<20$ \\
\hline LH $1000 \mathrm{C}$ & $<10$ & $N$ & 500 & 700 & 30 & $<50$ & 70 & 2,000 \\
\hline LH1001C & $<10$ & $N$ & 300 & 300 & 30 & $<50$ & 100 & 3,000 \\
\hline LH1002C & N & $N$ & 300 & 200 & 50 & $<50$ & 20 & 300 \\
\hline LH1003C & $N$ & $N$ & 300 & 150 & 700 & 50 & $N$ & 700 \\
\hline
\end{tabular}


TABLE 2. GEOCHEMICAL DATA FOR NONMAGNETIC, hEAVY-MINERAL-CONCENTRATE SAMPLES, LIME HILLS QUADRANGLE, ALASKA--CONT InUEd Sample Sc ppm-s Sn ppm-s Sr ppm-s Th ppm-s $U$ ppm-s $V$ ppm-s w ppm-s $\quad$ i ppm-s Zn ppm-s Zr ppm-s

\begin{tabular}{|c|c|c|c|c|c|c|c|c|c|c|}
\hline LH0954C & 50 & 150 & $<200$ & 2,000 & 1,500 & 150 & 500 & 700 & N & $>2,000$ \\
\hline LH0955C & 70 & 700 & $<200$ & 200 & 1,000 & 100 & 2,000 & 700 & N & $>2,000$ \\
\hline LH0956C & 50 & 30 & 200 & $<200$ & N & 150 & 100 & 300 & 1,000 & $>2,000$ \\
\hline LH0957C & 50 & 30 & 200 & N & $\mathbf{N}$ & 150 & 2,000 & 700 & N & $>2,000$ \\
\hline LH0958C & 50 & 30 & $<200$ & 200 & N & 150 & 200 & 500 & $\mathbf{N}$ & $>2,000$ \\
\hline LH0959C & 70 & N & N & $>5,000$ & 7,000 & 20 & 700 & 1,000 & $\mathbf{N}$ & $>2,000$ \\
\hline LH0960C & 30 & 20 & 700 & 700 & N & 150 & 150 & 300 & $\mathbf{N}$ & $>2,000$ \\
\hline LH0961C & 15 & $\mathbf{N}$ & 300 & N & N & 150 & N & 150 & $\mathbf{N}$ & $>2,000$ \\
\hline LH0962C & 15 & $N$ & $<200$ & N & N & 150 & 100 & 150 & $N$ & $>2,000$ \\
\hline LH0963C & $<10$ & 700 & 2,000 & 200 & $N$ & 20 & 150 & 150 & $\mathbf{N}$ & $>2,000$ \\
\hline LH0964C & 50 & 500 & 300 & N & N & 300 & N & 200 & N & $>2,000$ \\
\hline LH0965C & $N$ & N & 5,000 & $N$ & $N$ & 30 & $N$ & 50 & N & 1,500 \\
\hline LH0966C & 30 & 100 & 200 & $N$ & $N$ & 150 & $N$ & 200 & N & $>2,000$ \\
\hline LH0967C & 15 & 150 & 3,000 & N & $N$ & 150 & N & 100 & N & $>2,000$ \\
\hline LH0968C & 20 & 100 & 300 & $N$ & $N$ & 500 & N & 100 & $N$ & $>2,000$ \\
\hline LH0969C & 70 & $>2,000$ & $<200$ & 700 & 1,500 & 100 & 300 & 700 & $N$ & $>2,000$ \\
\hline LH0970C & 30 & 2,000 & 300 & 500 & $<1,000$ & 150 & 100 & 500 & $N$ & $>2,000$ \\
\hline LH0971C & $<10$ & N & 500 & $N$ & N & 50 & $N$ & 300 & N & $>2,000$ \\
\hline LH0972C & 50 & N & 200 & N & N & 150 & N & 300 & $N$ & $>2,000$ \\
\hline LH0973C & 30 & N & $<200$ & N & N & 70 & 100 & 500 & N & $>2,000$ \\
\hline LH0974C & 70 & 100 & $<200$ & $>5,000$ & 5,000 & 70 & 1,500 & 700 & N & $>2,000$ \\
\hline LH0975C & 20 & 30 & 300 & 700 & $N$ & 200 & 500 & 300 & N & $>2,000$ \\
\hline LH0976C & 10 & $\mathbf{N}$ & 700 & N & $N$ & 70 & $N$ & 100 & N & $>2,000$ \\
\hline LH0977C & $N$ & N & $<200$ & N & $\mathbf{N}$ & 20 & $N$ & 20 & $N$ & 2,000 \\
\hline LH0978C & 15 & $N$ & $<200$ & 200 & N & 100 & 50 & 200 & N & $>2,000$ \\
\hline LH0979C & 30 & 30 & $<200$ & 700 & N & 70 & N & 500 & N & $>2,000$ \\
\hline LH0980C & 30 & $N$ & 200 & 700 & 1,000 & 70 & 700 & 300 & N & $>2,000$ \\
\hline LH0981C & $<10$ & N & 300 & N & N & 100 & 200 & 200 & N & $>2,000$ \\
\hline LH0982C & 20 & N & 200 & $N$ & N & 200 & 100 & 70 & $N$ & $>2,000$ \\
\hline LH0983C & 20 & 150 & 200 & 700 & $<1,000$ & 150 & 100 & 500 & $N$ & $>2,000$ \\
\hline LH0984C & 50 & N & 500 & N & N & 700 & N & 100 & N & $>2,000$ \\
\hline LH0985C & 70 & 300 & 500 & N & N & 700 & $\mathbf{N}$ & 100 & N & $>2,000$ \\
\hline LH0986C & 70 & 300 & 700 & $N$ & $N$ & 500 & $\mathbf{N}$ & 100 & $N$ & $>2,000$ \\
\hline LH0987C & 100 & 50 & 500 & $N$ & N & 500 & N & 150 & $N$ & $>2,000$ \\
\hline LH0988C & 15 & $N$ & 300 & $N$ & $N$ & 150 & $N$ & 50 & 500 & $>2,000$ \\
\hline LH0989C & 50 & N & 200 & N & N & 300 & $N$ & 100 & N & $>2,000$ \\
\hline LH0990C & 20 & N & 1,000 & N & N & 200 & N & 100 & $N$ & $>2,000$ \\
\hline LH0991C & 20 & 70 & 700 & N & N & 200 & $\mathbf{N}$ & 200 & N & $>2,000$ \\
\hline LH0992C & 30 & 50 & 1,000 & N & N & 300 & N & 150 & $N$ & $>2,000$ \\
\hline LH0993C & 20 & 30 & 300 & N & N & 300 & $N$ & 100 & $N$ & $>2,000$ \\
\hline LH0994C & 15 & 20 & 1,500 & N & N & 200 & N & 100 & $N$ & $>2,000$ \\
\hline LH0995C & N & N & 7,000 & N & $N$ & 20 & $N$ & 30 & N & 2,000 \\
\hline LH0996C & $\mathbf{N}$ & 150 & 7,000 & N & N & 20 & 50 & 100 & $\mathbf{N}$ & $>2,000$ \\
\hline LH0997C & 70 & 500 & 700 & 200 & $N$ & 150 & 50 & 500 & N & $>2,000$ \\
\hline LH0998C & 20 & $>2,000$ & 1,000 & 500 & N & 150 & 300 & 500 & $N$ & $>2,000$ \\
\hline LH0999C & 50 & $>2,000$ & 500 & 1,500 & 1,500 & 150 & 300 & 700 & N & $>2,000$ \\
\hline LH1000C & 30 & 1,000 & $<200$ & $>5,000$ & 7,000 & 150 & 100 & 1,500 & N & $>2,000$ \\
\hline LH1001C & 30 & 200 & $<200$ & 1,500 & 2,000 & 150 & 150 & 1,000 & $\mathbf{N}$ & $>2,000$ \\
\hline LH1002C & 30 & 700 & N & $>5,000$ & 7,000 & N & 200 & 1,500 & N & $>2,000$ \\
\hline LH1003C & 50 & 1,500 & N & $>5,000$ & 7,000 & N & 1,500 & 1,500 & 2,000 & $>2,000$ \\
\hline
\end{tabular}


TABLE 2. GEOCHEMICAL DATA FOR NOMMAGMETIC, hEAVY-MIMERAL-CONCENTRATE SAMPLES, LIME hILlS QUADRAMGLE, ALASKA--CONT inUed

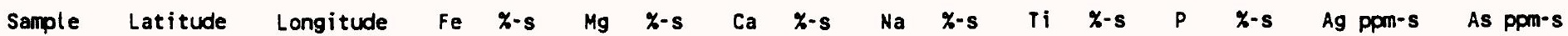

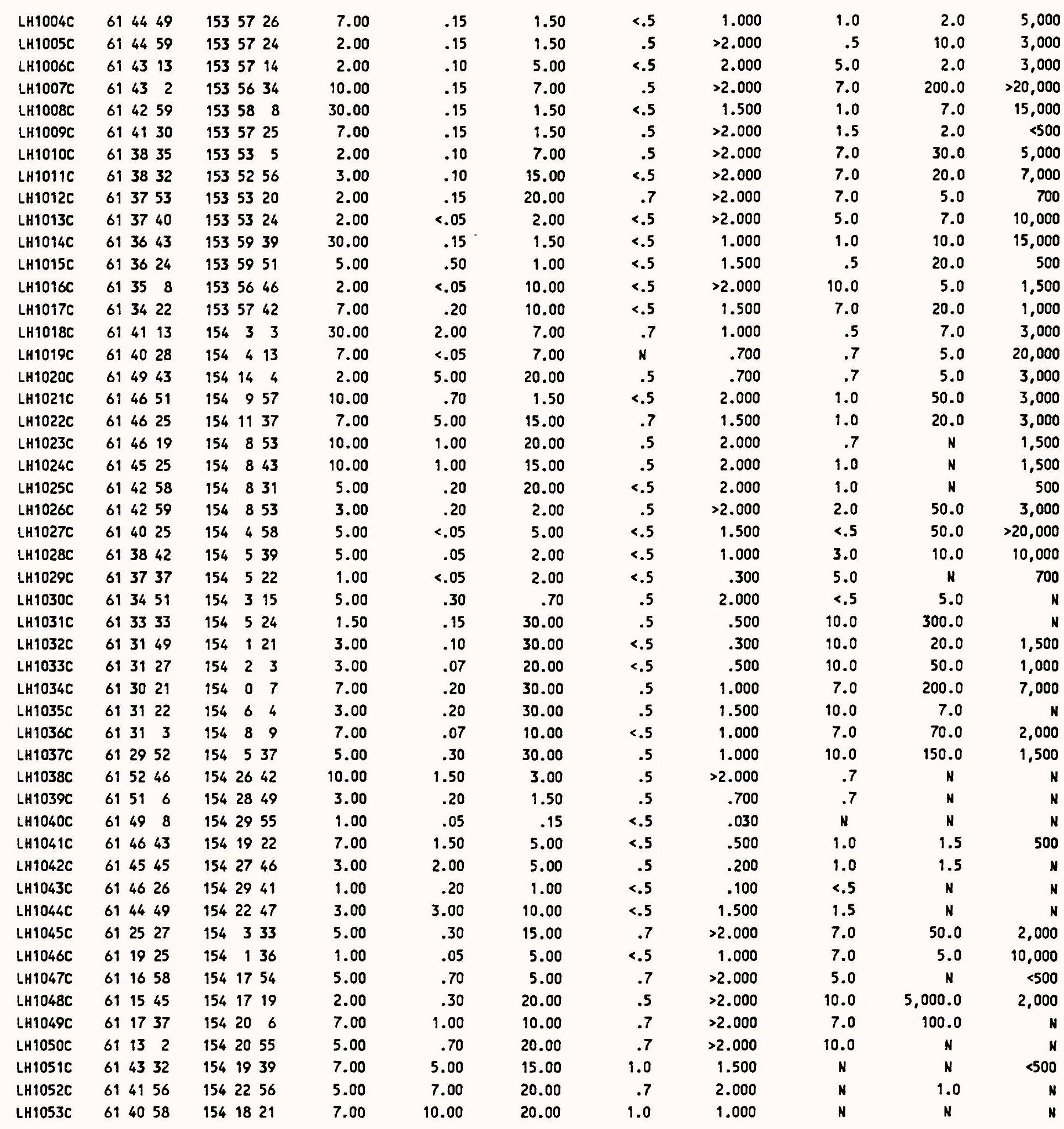




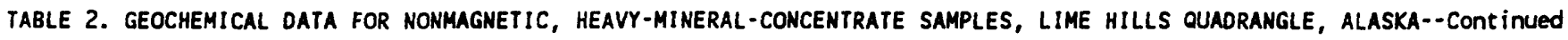

Sample Au ppm-s B ppm-s Ba ppm-s Be ppm-s Bi ppm-s Cd ppm-s Co ppm-s Cr ppm-s Cu ppm-s

\begin{tabular}{|c|c|c|c|c|c|c|c|c|c|}
\hline LH1004C & $N$ & 100 & 150 & 7 & N & N & 200 & 20 & 70 \\
\hline LH1005C & $\mathbf{N}$ & 70 & 3,000 & 50 & 500 & $N$ & 70 & $<20$ & 70 \\
\hline LH1006C & $\mathbf{N}$ & $N$ & 1,000 & 2 & 50 & N & 300 & 150 & 100 \\
\hline LH1007C & N & $\mathbf{N}$ & 2,000 & 50 & 500 & $N$ & 300 & 20 & 300 \\
\hline LH $1008 \mathrm{C}$ & $\mathbf{N}$ & 50 & 1,500 & $N$ & N & $N$ & 300 & 70 & 200 \\
\hline LH1009C & $N$ & 100 & 300 & 50 & 150 & N & 30 & 70 & 150 \\
\hline LH1010C & N & N & 300 & 3 & 100 & $N$ & 200 & $<20$ & 100 \\
\hline LH1011C & N & 100 & 150 & 3 & 500 & $N$ & 150 & $<20$ & 150 \\
\hline LH1012C & N & 1,000 & 150 & 7 & 500 & $N$ & 100 & $<20$ & 150 \\
\hline LH1013C & N & 30 & 70 & 2 & 100 & $N$ & 500 & $<20$ & 70 \\
\hline LH1014C & $N$ & $N$ & 1,500 & $N$ & N & $N$ & 500 & 50 & 500 \\
\hline LH1015C & $N$ & 100 & 1,500 & 3 & 30 & N & 100 & 200 & 100 \\
\hline LH1016C & $\mathbf{N}$ & N & 150 & $N$ & $\mathbf{N}$ & $N$ & 100 & $<20$ & 70 \\
\hline LH1017C & $N$ & 50 & 1,500 & $N$ & $N$ & N & 300 & 100 & 150 \\
\hline LH1018C & $N$ & 50 & $>10,000$ & $N$ & N & $N$ & 300 & 500 & 500 \\
\hline LH1019C & N & N & 200 & 5 & N & $N$ & 500 & $<20$ & 100 \\
\hline LH1020C & N & 100 & 1,500 & 50 & N & N & 200 & 50 & 70 \\
\hline LH1021C & $N$ & 20 & $>10,000$ & $\mathbf{N}$ & 100 & $N$ & 150 & 20 & 100 \\
\hline LH1022C & $\mathbf{N}$ & 300 & 5,000 & 3 & N & $N$ & 100 & 150 & 150 \\
\hline LH1023C & N & 50 & $>10,000$ & 5 & $N$ & $N$ & 100 & 200 & 200 \\
\hline LH1024C & $N$ & 50 & $>10,000$ & 5 & $N$ & N & 70 & 150 & 150 \\
\hline LH1025C & N & 20 & 700 & 100 & N & N & 30 & 100 & 20 \\
\hline LH1026C & $N$ & $N$ & 300 & 3 & $N$ & N & 100 & 50 & 500 \\
\hline LK1027C & $N$ & $N$ & 500 & 3 & $N$ & $N$ & 700 & $<20$ & 70 \\
\hline LH1028C & $N$ & $N$ & 200 & $N$ & $N$ & H & 500 & $<20$ & 100 \\
\hline LH1029C & $N$ & 20 & 150 & $N$ & $N$ & N & 20 & $<20$ & 50 \\
\hline LH1030C & N & 150 & 700 & 3 & $N$ & N & 30 & 200 & 100 \\
\hline LH1031C & $N$ & 70 & 300 & 2 & N & N & 20 & 70 & 10 \\
\hline LH1032C & $N$ & 50 & 200 & N & N & $N$ & 30 & $<20$ & 200 \\
\hline LH1033C & $N$ & 20 & 150 & N & N & $N$ & 70 & $<20$ & 100 \\
\hline LH1034C & $N$ & 100 & 200 & 3 & 30 & $N$ & 200 & 30 & 150 \\
\hline LH1035C & N & 200 & 300 & 2 & 30 & N & 50 & 70 & 150 \\
\hline LH1036C & $N$ & N & 150 & 3 & 50 & N & 50 & $<20$ & 100 \\
\hline LH1037C & N & 200 & 200 & $N$ & $N$ & N & 70 & 20 & . 70 \\
\hline LH1038C & $N$ & 50 & $>10,000$ & 3 & N & N & 30 & 500 & 150 \\
\hline LH1039C & $N$ & 50 & $>10,000$ & N & $N$ & 200 & $<20$ & 70 & 70 \\
\hline LH104OC & $N$ & $\mathbf{N}$ & $>10,000$ & N & N & N & $<20$ & $<20$ & 10 \\
\hline LH1041C & $N$ & 50 & $>10,000$ & N & N & 100 & 30 & 70 & 70 \\
\hline LH1042C & $N$ & 20 & $>10,000$ & N & N & N & $<20$ & 1,000 & 20 \\
\hline LH1043C & $N$ & $N$ & $>10,000$ & N & $N$ & $N$ & $<20$ & 150 & 10 \\
\hline LH1044C & $N$ & 1,000 & $>10,000$ & N & $\mathbf{N}$ & $N$ & $<20$ & 200 & 30 \\
\hline LH1045C & N & 1,500 & 1,500 & 70 & 70 & $N$ & 100 & 30 & 100 \\
\hline LH1046C & N & 50 & 700 & 2 & 150 & N & 500 & $<20$ & 100 \\
\hline LH1047C & N & 1,500 & 700 & 7 & 30 & N & 30 & 150 & 30 \\
\hline LK1048C & $>1,000$ & 2,000 & 500 & 3 & 30 & $N$ & 30 & 70 & 30 \\
\hline LH1049C & 150 & 5,000 & 700 & 5 & 200 & N & 20 & 150 & 150 \\
\hline LH1050C & N & 500 & 300 & 2 & N & $N$ & $<20$ & 150 & 30 \\
\hline LH1051C & $N$ & 1,000 & 700 & 3 & N & $N$ & 30 & 300 & 50 \\
\hline LH1052C & $N$ & 3,000 & 7,000 & 3 & 70 & $\mathbf{N}$ & 20 & 200 & 70 \\
\hline ᄂH1053C & $N$ & 300 & 3,000 & 2 & $N$ & $\mathbf{N}$ & 20 & 150 & 100 \\
\hline
\end{tabular}




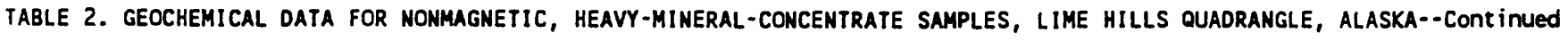

Sample Ga ppm-s Ge ppm-s La ppm-s Mn ppm-s Mo ppm-s Nb ppm-s Ni ppm-s Pb ppm-s Pt ppm-s Sb ppm-s

\begin{tabular}{|c|c|c|c|c|c|c|c|c|c|}
\hline LH1004C & $<10$ & N & 1,000 & 200 & 30 & 50 & 150 & 200 & $N$ \\
\hline LH1005C & $<10$ & N & 700 & 200 & 500 & 100 & 30 & 2,000 & N \\
\hline LH1006C & 10 & $N$ & 700 & 1,000 & 10 & $<50$ & 200 & 300 & $N$ \\
\hline LH1007C & 10 & N & 1,000 & 1,000 & 10 & 200 & 200 & 20,000 & $\mathbf{N}$ \\
\hline LH1008C & 10 & $N$ & 500 & 500 & $N$ & 50 & 500 & 1,000 & $N$ \\
\hline LH1009C & 100 & N & 150 & 1,000 & $N$ & 200 & 70 & 100 & N \\
\hline LH1010C & 10 & N & 1,000 & 2,000 & N & 100 & 70 & 700 & $\mathbf{N}$ \\
\hline LH1011C & $<10$ & $N$ & 1,500 & 3,000 & N & 100 & 70 & 500 & $\mathbf{N}$ \\
\hline LH1012C & 10 & $N$ & 700 & 7,000 & $N$ & 150 & 70 & 100 & N \\
\hline LH1013C & $<10$ & $N$ & 1,000 & 2,000 & N & 50 & 200 & 150 & $N$ \\
\hline LH1014C & $<10$ & $N$ & 700 & 500 & N & $<50$ & 700 & 1,000 & $N$ \\
\hline LH1015C & 30 & N & 100 & 500 & N & 50 & 100 & 700 & N \\
\hline LH1016C & $<10$ & $N$ & 1,000 & 1,000 & N & 100 & 100 & 150 & $N$ \\
\hline LH1017C & 15 & $N$ & 2,000 & 1,000 & 15 & $<50$ & 300 & 1,000 & $N$ \\
\hline LH1018C & 15 & $N$ & 1,500 & 1,000 & N & $<50$ & 700 & 1,000 & $N$ \\
\hline LH1019C & $<10$ & $N$ & 1,000 & 300 & N & 100 & 500 & 200 & N \\
\hline LH1020C & 10 & $N$ & 200 & 700 & N & $<50$ & 150 & 200 & N \\
\hline LH1021C & $<10$ & $N$ & 700 & 200 & 100 & 100 & 150 & 3,000 & $N$ \\
\hline LH1022C & 15 & $N$ & 300 & 1,000 & 50 & $<50$ & 100 & 2,000 & $N$ \\
\hline LH $1023 \mathrm{C}$ & 20 & $N$ & 2,000 & 1,000 & N & 100 & 100 & 100 & $\mathbf{N}$ \\
\hline LH1024C & 15 & $N$ & 1,500 & 1,000 & $N$ & 100 & 100 & 100 & N \\
\hline LH1025C & $<10$ & N & $>2,000$ & 2,000 & $N$ & 100 & 10 & 100 & $\mathbf{N}$ \\
\hline LH1026C & 10 & N & 2,000 & 1,000 & 20 & 70 & 100 & 2,000 & N \\
\hline LH1027C & N & $\mathbf{N}$ & 700 & 200 & 10 & 50 & 1,000 & 200 & $N$ \\
\hline LH1028C & $<10$ & $N$ & 1,000 & 1,000 & 70 & $<50$ & 1,000 & 700 & N \\
\hline LH1029C & $<10$ & $\mathbf{N}$ & 1,500 & 1,000 & N & $<50$ & 20 & 30 & N \\
\hline LH1030C & 30 & N & 150 & 1,500 & $\mathbf{N}$ & 50 & 70 & 300 & $\mathbf{N}$ \\
\hline LH1031C & 15 & $N$ & 1,500 & 2,000 & N & $<50$ & 15 & 70 & N \\
\hline LH1032C & $<10$ & $N$ & $>2,000$ & 1,000 & 15 & $<50$ & 70 & 700 & N \\
\hline LH1033C & $<10$ & N & $>2,000$ & 1,000 & 20 & N & 50 & 700 & $N$ \\
\hline LH1034C & 10 & N & 1,500 & 2,000 & 20 & $<50$ & 150 & 3,000 & N \\
\hline LH1035C & 10 & N & $>2,000$ & 1,500 & $N$ & 50 & 30 & 300 & N \\
\hline LH1036C & $<10$ & N & 2,000 & 1,000 & 20 & $<50$ & 50 & 1,000 & N \\
\hline LH1037C & 10 & N & $>2,000$ & 5,000 & $N$ & $<50$ & 70 & 700 & N \\
\hline LH1038C & $<10$ & N & 300 & 1,000 & $\mathbf{N}$ & 50 & 70 & 30 & N \\
\hline LH1039C & 10 & $N$ & 150 & 70 & N & $<50$ & 30 & 20 & N \\
\hline LH1040C & N & $N$ & N & 100 & N & $<50$ & 20 & $<20$ & N \\
\hline LH1041C & $<10$ & $N$ & N & 200 & $N$ & $<50$ & 70 & 150 & N \\
\hline LH1042C & $<10$ & $N$ & N & 300 & $N$ & $<50$ & 70 & 20 & N \\
\hline LH1043C & N & $N$ & N & 100 & $N$ & $<50$ & 15 & $<20$ & N \\
\hline LH1044C & 15 & N & 100 & 1,000 & N & $<50$ & 30 & $<20$ & N \\
\hline LH $1045 \mathrm{C}$ & 20 & N & 2,000 & 5,000 & 30 & 200 & 100 & 200 & N \\
\hline LH1046C & N & N & $>2,000$ & 3,000 & $N$ & $<50$ & 500 & 30 & N \\
\hline LH1047C & 10 & N & 1,500 & 5,000 & N & 200 & 20 & 30 & N \\
\hline LH1048C & 10 & N & 1,000 & 7,000 & N & 100 & 30 & 20 & N \\
\hline LH1049C & 70 & N & 300 & 3,000 & N & 200 & 70 & 30 & $\mathbf{N}$ \\
\hline LH1050C & $<10$ & $N$ & 2,000 & 5,000 & N & 70 & 20 & 20 & $N$ \\
\hline LH1051C & 20 & N & 2,000 & 2,000 & N & $<50$ & 70 & $<20$ & N \\
\hline LH1052C & 20 & N & 150 & 1,500 & N & 50 & 30 & 30 & N \\
\hline LH1053C & 10 & $\mathbf{N}$ & 200 & 2,000 & $N$ & 50 & 30 & $<20$ & N \\
\hline
\end{tabular}


TABLE 2. GEOChEMICAL DATA FOR NONMAGNETIC, hEAVy-MINERAL-CONCENTRATE SAMPLES, LIME hILLS QUADRANGLE, ALASKA--CONTinUEd

Sample Sc ppm-s Sn ppm-s Sr ppm-s Th ppm-s U ppm-s V ppm-s w ppm-s Y ppm-s Zn ppm-s Zr ppm-s

\begin{tabular}{|c|c|c|c|c|c|c|c|c|c|c|}
\hline LH1004C & 30 & 20 & N & $>5,000$ & 5,000 & 50 & 300 & 1,500 & 2,000 & $>2,000$ \\
\hline LH1005C & 50 & 1,000 & $<200$ & $>5,000$ & 5,000 & 50 & 2,000 & 1,000 & 1,000 & $>2,000$ \\
\hline LH $1006 C$ & 70 & 70 & $<200$ & 1,000 & 2,000 & 150 & 200 & 1,000 & N & $>2,000$ \\
\hline LH1007C & 70 & 700 & N & $<200$ & 2,000 & 70 & 2,000 & 700 & N & $>2,000$ \\
\hline LH1008C & 15 & $N$ & $N$ & $N$ & N & 100 & 10,000 & 200 & N & $>2,000$ \\
\hline LH1009C & 70 & 100 & $<200$ & N & N & 150 & 150 & 300 & N & $>2,000$ \\
\hline LH1010C & 200 & 2,000 & $<200$ & 200 & 3,000 & 50 & 1,000 & 700 & $N$ & $>2,000$ \\
\hline LH1011C & 150 & 2,000 & $<200$ & 200 & 3,000 & 50 & 2,000 & 700 & $N$ & $>2,000$ \\
\hline LH1012C & 150 & 500 & $<200$ & $<200$ & 3,000 & 30 & 500 & 700 & $N$ & $>2,000$ \\
\hline LH1013C & 200 & 200 & $N$ & 200 & 3,000 & 20 & 100 & 1,000 & $N$ & $>2,000$ \\
\hline LH1014C & 15 & 20 & $N$ & $N$ & N & 70 & 300 & 150 & 700 & $>2,000$ \\
\hline LH1015C & 10 & 150 & $<200$ & N & N & 200 & 200 & 70 & N & $>2,000$ \\
\hline LH1016C & 200 & 100 & $<200$ & $<200$ & 1,000 & 100 & 200 & 700 & N & $>2,000$ \\
\hline LH1017C & 150 & $N$ & $<200$ & 700 & 2,000 & 150 & N & 700 & 1,000 & $>2,000$ \\
\hline LH1018C & 50 & 20 & $N$ & N & $<1,000$ & 300 & 200 & 200 & N & $>2,000$ \\
\hline LH1019C & 100 & 500 & N & $>5,000$ & 7,000 & 30 & 200 & 1,000 & $\mathbf{N}$ & $>2,000$ \\
\hline LH1020C & 20 & 200 & 1,000 & 500 & 1,000 & 100 & 70 & 1,000 & $N$ & $>2,000$ \\
\hline LH1021C & 50 & 20 & 1,000 & 1,500 & 1,500 & 150 & 700 & 700 & N & $>2,000$ \\
\hline LH1022C & 30 & 1,500 & 700 & $N$ & N & 300 & 100 & 300 & $N$ & $>2,000$ \\
\hline LH1023C & 100 & 30 & 700 & 1,000 & 1,000 & 150 & 200 & 700 & $N$ & $>2,000$ \\
\hline LH1024C & 100 & 200 & 500 & 1,000 & 1,000 & 150 & 200 & 700 & $\mathbf{N}$ & $>2,000$ \\
\hline LH1025C & 200 & $N$ & $N$ & $>5,000$ & 3,000 & 150 & 200 & 3,000 & N & $>2,000$ \\
\hline LH1026C & 100 & 200 & N & 5,000 & 3,000 & 100 & 200 & 1,000 & 2,000 & $>2,000$ \\
\hline LH1027C & 100 & 200 & N & $>5,000$ & 7,000 & 20 & 300 & 1,000 & N & $>2,000$ \\
\hline LH1028C & 100 & 20 & N & 5,000 & 5,000 & 20 & 300 & 700 & $N$ & $>2,000$ \\
\hline LH1029C & 150 & 150 & $N$ & 1,000 & 1,000 & 20 & 100 & 1,000 & N & $>2,000$ \\
\hline LH1030C & 15 & $N$ & $<200$ & N & N & 200 & 200 & 100 & $N$ & $>2,000$ \\
\hline LH1031C & 70 & 150 & $<200$ & 200 & N & 70 & 70 & 1,000 & N & $>2,000$ \\
\hline LH1032C & 150 & 70 & 300 & 1,500 & 3,000 & 150 & 150 & 700 & N & $>2,000$ \\
\hline LH1033C & 200 & N & 200 & 700 & 2,000 & 20 & 100 & 1,000 & N & $>2,000$ \\
\hline LH1034C & 50 & 2,000 & 200 & $<200$ & 1,000 & 70 & 500 & 1,000 & $N$ & $>2,000$ \\
\hline LH1035C & 100 & 20 & 200 & 500 & 1,000 & 100 & 500 & 1,000 & $N$ & $>2,000$ \\
\hline LH1036C & 100 & 150 & $<200$ & 700 & N & 50 & 70 & 1,000 & $N$ & $>2,000$ \\
\hline LH1037C & 100 & 20 & 200 & 300 & $N$ & 50 & 50 & 2,000 & $N$ & $>2,000$ \\
\hline LH1038C & 20 & 500 & 1,000 & N & $N$ & 150 & 100 & 300 & N & $>2,000$ \\
\hline LH1039C & $N$ & 500 & 3,000 & $N$ & $N$ & 50 & $N$ & 70 & 15,000 & $>2,000$ \\
\hline LH1040C & $N$ & N & 3,000 & $N$ & N & 50 & $N$ & $N$ & N & 150 \\
\hline LH1041C & $<10$ & 100 & 2,000 & N & N & 150 & $N$ & 30 & 7,000 & 1,500 \\
\hline LH1042C & 15 & 20 & 5,000 & N & N & 150 & $\mathbf{N}$ & 100 & N & $>2,000$ \\
\hline LH1043C & $N$ & 2,000 & $>10,000$ & N & N & 20 & $N$ & 30 & $N$ & 2,000 \\
\hline LH1044C & 15 & 300 & 3,000 & $N$ & N & 700 & N & 100 & 700 & $>2,000$ \\
\hline LH1045C & 150 & $>2,000$ & $<200$ & 300 & 1,500 & 100 & 70 & 1,500 & $N$ & $>2,000$ \\
\hline LH1046C & 200 & 2,000 & N & 500 & 5,000 & 20 & 100 & 1,500 & N & $>2,000$ \\
\hline LH1047C & 150 & $>2,000$ & $N$ & 200 & 1,000 & 70 & 150 & 700 & $N$ & $>2,000$ \\
\hline LH1048C & 100 & $>2,000$ & $<200$ & $<200$ & N & 100 & 150 & 1,500 & $N$ & $>2,000$ \\
\hline LH1049C & 70 & 1,000 & 700 & N & N & 300 & 1,000 & 300 & $N$ & $>2,000$ \\
\hline LH1050C & 200 & $>2,000$ & $<200$ & $<200$ & $<1,000$ & 150 & 1,000 & 1,000 & $N$ & $>2,000$ \\
\hline LH1051C & 150 & 300 & 500 & 200 & N & 300 & N & 100 & $\mathbf{N}$ & $>2,000$ \\
\hline LH1052C & 30 & 150 & 700 & N & $N$ & 500 & 100 & 100 & $N$ & 2,000 \\
\hline LH1053C & 30 & $N$ & 300 & $N$ & $N$ & 700 & $N$ & 150 & $N$ & $>2,000$ \\
\hline
\end{tabular}




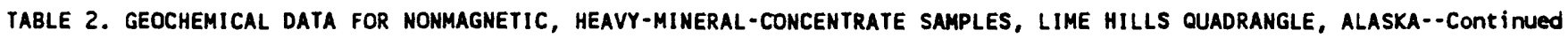

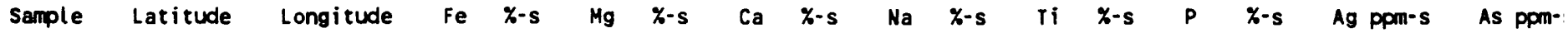

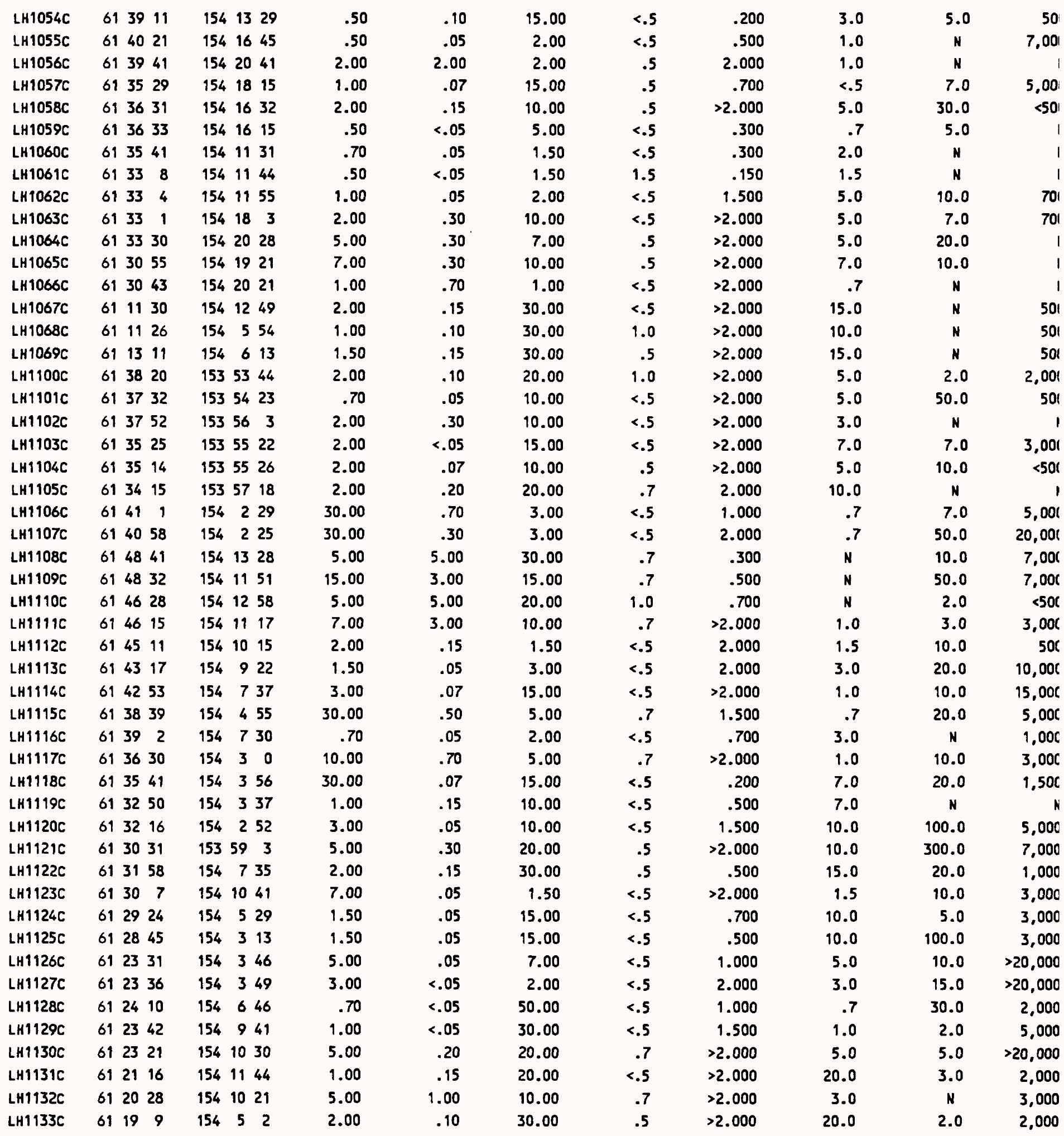




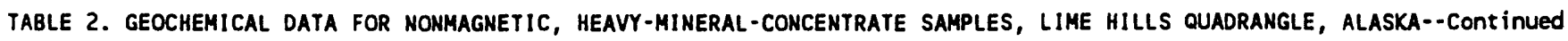

Sample AU ppm-s B ppm-s Ba ppm-s Be ppm-s Bi ppm-s Co ppm-s Co ppm-s Cr ppm-s CU ppm-s

\begin{tabular}{|c|c|c|c|c|c|c|c|c|c|}
\hline LH1054C & N & $N$ & 200 & 3 & 500 & N & 30 & $<20$ & 50 \\
\hline LH1055C & $N$ & N & 1,000 & 3 & 500 & N & 50 & $<20$ & 30 \\
\hline LH1056C & $N$ & 70 & 200 & 2 & N & $N$ & 30 & 200 & 10 \\
\hline LH1057C & $N$ & $N$ & 200 & 7 & 200 & $N$ & 100 & $<20$ & 50 \\
\hline LH1058C & 50 & $N$ & 700 & 3 & N & $N$ & 30 & 50 & 15 \\
\hline LH1059C & $N$ & $N$ & 500 & 5 & 500 & $N$ & 70 & $<20$ & 70 \\
\hline LH1060C & $N$ & $N$ & 300 & 15 & 200 & $N$ & 30 & $<20$ & 150 \\
\hline LH1061C & $N$ & N & 1,000 & 2 & $N$ & $N$ & N & $<20$ & 20 \\
\hline LH1062C & N & N & 300 & N & N & N & $<20$ & $<20$ & 50 \\
\hline LH1063C & N & 70 & 7,000 & 70 & 500 & $N$ & 20 & 50 & 50 \\
\hline LH $1064 \mathrm{C}$ & $N$ & 20 & 1,000 & N & $N$ & $N$ & 20 & 150 & 70 \\
\hline LH1065C & $\mathbf{N}$ & $N$ & $>10,000$ & N & $N$ & $N$ & 50 & 70 & 100 \\
\hline LH1066C & $N$ & 50 & 500 & 3 & N & $N$ & $<20$ & 150 & 20 \\
\hline LH1067C & $\mathbf{N}$ & 100 & $>10,000$ & $N$ & 700 & $N$ & 30 & 70 & 100 \\
\hline LH1068C & $\mathbf{N}$ & 30 & 700 & 2 & N & $N$ & 50 & 30 & $<10$ \\
\hline LH1069C & $N$ & 1,500 & 150 & 2 & N & $N$ & $<20$ & 30 & 50 \\
\hline LH1100C & $N$ & N & 300 & 3 & 50 & $N$ & 30 & 20 & 100 \\
\hline LH1101C & $N$ & $N$ & 300 & 2 & $<20$ & N & 30 & $<20$ & 70 \\
\hline LH1102C & $N$ & 100 & 500 & 10 & $<20$ & $N$ & $<20$ & 500 & 70 \\
\hline LH1103C & $N$ & 20 & 100 & 15 & $<20$ & $N$ & 70 & $<20$ & 150 \\
\hline LH1104C & $N$ & $N$ & 300 & 2 & $N$ & N & 70 & 20 & 100 \\
\hline LH1105C & $N$ & $N$ & 300 & 2 & $N$ & N & $<20$ & 30 & 70 \\
\hline LH1106C & N & $N$ & $>10,000$ & $N$ & $N$ & $N$ & 300 & 30 & 500 \\
\hline LH1107C & 150 & $N$ & $>10,000$ & $N$ & $N$ & $N$ & 700 & 100 & 700 \\
\hline LH1108C & N & 50 & 7,000 & 150 & 100 & N & 100 & 70 & 20 \\
\hline LH1109C & $N$ & 100 & $>10,000$ & $N$ & 500 & $N$ & 50 & 50 & 50 \\
\hline LH1110C & N & 150 & 3,000 & 2 & $N$ & $N$ & 20 & 150 & 70 \\
\hline LH1111C & $N$ & 200 & 7.000 & 2 & N & N & 150 & 100 & 150 \\
\hline LH1112C & $N$ & 50 & 700 & N & N & $N$ & $<20$ & 30 & 50 \\
\hline LH1113C & $N$ & $\mathbf{N}$ & 200 & 3 & N & $N$ & 500 & $<20$ & 100 \\
\hline LH1114C & N & N & 200 & 3 & 150 & N & 300 & $<20$ & 100 \\
\hline LH1115C & $N$ & 200 & 1,000 & $\mathbf{N}$ & N & $N$ & 700 & 100 & 500 \\
\hline LH1116C & $N$ & N & 150 & $\mathbf{N}$ & N & $\mathbf{N}$ & 30 & $<20$ & 70 \\
\hline LH1117C & $N$ & 1,000 & 1,000 & 2 & $N$ & $\mathbf{N}$ & 700 & 150 & 700 \\
\hline LH1118C & $N$ & N & 300 & $N$ & $N$ & $N$ & 500 & 20 & 200 \\
\hline LH1119C & $N$ & 70 & 500 & 7 & $N$ & $N$ & $<20$ & 150 & 15 \\
\hline LH1120C & $N$ & N & 150 & $N$ & 150 & $N$ & 300 & $<20$ & 150 \\
\hline LH1121C & $N$ & 100 & 300 & N & $N$ & $N$ & 20 & 50 & 150 \\
\hline LH1122C & $N$ & 20 & 200 & N & $N$ & $N$ & 70 & $<20$ & 30 \\
\hline LH1123C & N & N & $>10,000$ & 3 & 500 & $N$ & 200 & $<20$ & 100 \\
\hline LH1124C & $N$ & 100 & 300 & $N$ & N & $N$ & 200 & $<20$ & 100 \\
\hline LH1125C & $N$ & $N$ & 70 & $N$ & $<20$ & N & 300 & $<20$ & 150 \\
\hline LH1126C & $N$ & 200 & 50 & 7 & 200 & $N$ & 2,000 & $<20$ & 100 \\
\hline LH1127C & $N$ & 50 & 150 & 3 & 150 & $N$ & 2,000 & $<20$ & 100 \\
\hline LH $128 \mathrm{C}$ & N & 70 & 70 & 7 & N & $N$ & 70 & $<20$ & 10 \\
\hline LH1129C & N & N & 200 & 7 & N & N & 100 & $<20$ & 10 \\
\hline LH1130C & $N$ & 500 & 70 & 15 & 200 & N & 700 & 50 & 50 \\
\hline LH $1131 \mathrm{C}$ & $N$ & 50 & 70 & 3 & 70 & $N$ & 70 & 30 & 100 \\
\hline LH1132C & $N$ & 2,000 & 70 & 15 & N & N & 100 & 100 & 20 \\
\hline LH1133C & $N$ & 200 & 70 & 5 & 30 & $N$ & 100 & 20 & 100 \\
\hline
\end{tabular}




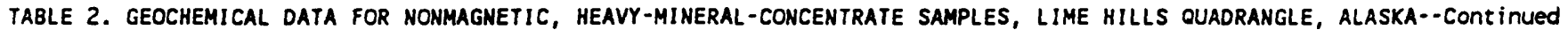

Sample Ga ppm-s Ge ppm-s La ppm-s Mn ppm-s Mo ppm-s Nb ppm-s Ni ppm-s Pb ppm-s Pt ppm-s Sb ppm-s

\begin{tabular}{|c|c|c|c|c|c|c|c|c|}
\hline LH1054C & $N$ & $N$ & 1,000 & 300 & 20 & 50 & N & 200 \\
\hline LH1055C & $N$ & $N$ & 300 & 200 & $N$ & $<50$ & 200 & 200 \\
\hline LH1056C & 20 & N & 2,000 & 1,000 & $N$ & 100 & 20 & 30 \\
\hline LH1057C & $<10$ & $N$ & 1,500 & 200 & $N$ & 100 & 150 & 500 \\
\hline LH1058C & 10 & $N$ & 700 & 1,000 & 50 & 50 & 15 & 1,000 \\
\hline LH1059C & $<10$ & $N$ & 2,000 & 300 & $N$ & 70 & N & 500 \\
\hline LH1060C & $<10$ & $N$ & $>2,000$ & 700 & $N$ & 70 & $<10$ & 150 \\
\hline LH1061C & 10 & $N$ & 100 & 150 & N & $<50$ & $<10$ & 20 \\
\hline LH1062C & $<10$ & $N$ & 500 & 300 & $N$ & $<50$ & 10 & 150 \\
\hline LH1063C & $<10$ & N & 300 & 1,000 & N & 100 & 50 & 700 \\
\hline LH1064C & $<10$ & $N$ & 500 & 500 & $N$ & 100 & 30 & $<20$ \\
\hline LH1065C & $<10$ & N & 500 & 500 & 15 & 150 & 70 & 2,000 \\
\hline LH1066C & $<10$ & N & 1,000 & 500 & $N$ & 50 & 20 & 70 \\
\hline LH1067C & $<10$ & $N$ & 1,000 & 7,000 & N & 150 & 50 & 20 \\
\hline LH1068C & 10 & $N$ & 500 & 7,000 & $N$ & 150 & 50 & 20 \\
\hline LH1069C & 10 & $N$ & 700 & 7,000 & $N$ & 150 & 10 & 20 \\
\hline LH1100C & 10 & $N$ & 700 & 1,500 & $N$ & 150 & 20 & 50 \\
\hline LH1101C & 10 & N & 500 & 500 & $N$ & 150 & 20 & 700 \\
\hline LH1102C & 30 & 20 & 300 & 1,500 & $N$ & 100 & 30 & $<20$ \\
\hline LH1103C & 10 & $N$ & 300 & 1,000 & N & 70 & 70 & 150 \\
\hline LH1104C & 10 & $N$ & 700 & 500 & N & 200 & 50 & 200 \\
\hline LH1105C & 15 & $N$ & 1,500 & 1,500 & $N$ & $<50$ & 20 & 50 \\
\hline LH1106C & 10 & $N$ & 150 & 100 & N & $<50$ & 300 & 1,500 \\
\hline LH1107C & $<10$ & $N$ & 100 & 100 & N & 50 & 700 & 5,000 \\
\hline LH1108C & $<10$ & $N$ & 300 & 300 & N & 50 & 100 & 1,000 \\
\hline LH1109C & $<10$ & $N$ & $N$ & 300 & 150 & $<50$ & 100 & 3,000 \\
\hline LH1110C & 10 & $N$ & 100 & 500 & N & $<50$ & 50 & 500 \\
\hline LH1111C & 10 & $N$ & 700 & 700 & 20 & 100 & 150 & 500 \\
\hline LH1912C & $<10$ & $N$ & 300 & 1,000 & 150 & $<50$ & 30 & 70 \\
\hline LH1113C & $<10$ & $N$ & 700 & 700 & N & 50 & 200 & 300 \\
\hline LH1114C & $<10$ & $N$ & 1,000 & 150 & $N$ & 100 & 200 & 300 \\
\hline LH1115C & 10 & $N$ & 700 & 1,000 & N & $<50$ & 700 & 1,500 \\
\hline LH1116C & $<10$ & $N$ & 500 & 300 & $N$ & $<50$ & 50 & 20 \\
\hline LH1117C & 10 & N & 500 & 1,500 & 30 & 70 & 300 & 3,000 \\
\hline LH1118C & $<10$ & $N$ & 700 & 1,000 & N & $<50$ & 500 & 2,000 \\
\hline LH1119C & 20 & $N$ & 700 & 700 & $N$ & $<50$ & 20 & 20 \\
\hline LH1120C & $<10$ & N & 1,000 & 1,500 & N & 50 & 300 & 1,500 \\
\hline LH1121C & $<10$ & $N$ & 1,000 & 1,500 & 300 & 70 & 20 & 2,000 \\
\hline LH1122C & $<10$ & $N$ & 1,500 & 1,500 & N & $<50$ & 50 & 1,000 \\
\hline LH1123C & $<10$ & $N$ & 500 & 300 & 50 & 100 & 100 & 300 \\
\hline LH1124C & $<10$ & $N$ & 2,000 & 3,000 & $N$ & $<50$ & 150 & 200 \\
\hline LH1125C & $<10$ & $N$ & 1,500 & 3,000 & N & $<50$ & 100 & 150 \\
\hline LH1126C & $<10$ & N & $>2,000$ & 3,000 & N & 50 & 1,500 & 150 \\
\hline LH1127C & $<10$ & $N$ & $>2,000$ & 1,000 & N & 70 & 1,500 & 700 \\
\hline LH1128C & $<10$ & $N$ & 1,500 & 300 & N & 100 & 30 & 500 \\
\hline LH1129C & $<10$ & $N$ & 1,500 & 500 & $N$ & 100 & 50 & 500 \\
\hline LH1130C & 10 & $N$ & 2,000 & 5,000 & $N$ & 100 & 700 & 200 \\
\hline LH1131C & $<10$ & $N$ & 1,500 & 7,000 & 20 & 100 & 70 & 300 \\
\hline LH1132C & 30 & $<20$ & 700 & 2,000 & N & 300 & 70 & 50 \\
\hline LH1133C & $<10$ & N & 2,000 & 7,000 & $N$ & 70 & 100 & 70 \\
\hline
\end{tabular}


TABLE 2. GEOCHEMICAL DATA FOR NONMAGNETIC, hEAVY-MINERAL-CONCENTRATE SAMPLES, LIME hILLS QUADRANGLE, ALASKA--Cont InUEd

Sample Sc ppm-s Sn ppm-s sr ppm-s Th ppm-s $U$ ppm-s $V$ ppm-s $\quad W$ ppm-s $\quad$ Y ppm-s Zn ppm-s Zr ppm-s

\begin{tabular}{|c|c|c|c|c|c|c|c|c|c|c|}
\hline LH1054C & 30 & 500 & N & $>5,000$ & 15,000 & 30 & 1,000 & $>5,000$ & N & $>2,000$ \\
\hline LH1055C & 30 & 700 & N & $>5,000$ & 15,000 & 20 & 200 & $>5,000$ & N & $>2,000$ \\
\hline LH1056C & 50 & 150 & $<200$ & 3,000 & 3,000 & 150 & 70 & 1,500 & N & $>2,000$ \\
\hline LH1057C & 50 & $>2,000$ & $<200$ & $>5,000$ & 15,000 & 150 & 1,000 & 1,500 & $N$ & $>2,000$ \\
\hline LH1058C & 30 & 200 & $<200$ & 2,000 & 2,000 & 150 & 50 & 700 & N & $>2,000$ \\
\hline LH1059C & 50 & $>2,000$ & $<200$ & $>5,000$ & 20,000 & 30 & 1,000 & 5,000 & N & $>2,000$ \\
\hline LH1060C & 50 & $>2,000$ & N & $>5,000$ & 15,000 & 20 & 1,000 & 3,000 & N & $>2,000$ \\
\hline LH1061C & 30 & 30 & $<200$ & 1,000 & 1,000 & $<20$ & 100 & 300 & $N$ & $>2,000$ \\
\hline LH1062C & 100 & 50 & N & 1,000 & 1,000 & 20 & 50 & 700 & N & $>2,000$ \\
\hline LH1063C & 50 & $>2,000$ & 500 & 300 & $N$ & 100 & 15,000 & 500 & $N$ & $>2,000$ \\
\hline LH1064C & 150 & 100 & 700 & $<200$ & $N$ & 300 & 5,000 & 500 & $N$ & $>2,000$ \\
\hline LH1065C & 70 & 700 & 1,000 & $<200$ & H & 150 & 50 & 300 & $N$ & $>2,000$ \\
\hline LH1066C & 150 & 500 & $<200$ & 700 & 1,000 & 150 & 100 & 500 & $N$ & $>2,000$ \\
\hline LH1067C & 150 & 2,000 & 700 & $N$ & $N$ & 100 & 100 & 700 & $N$ & $>2,000$ \\
\hline LH1068C & 70 & 700 & $<200$ & N & $N$ & 30 & 200 & 700 & $N$ & $>2,000$ \\
\hline LH1069C & 70 & 700 & $<200$ & N & $N$ & 100 & 100 & 1,000 & $N$ & $>2,000$ \\
\hline LH1100C & 150 & 150 & $<200$ & N & $N$ & 50 & 500 & 1,000 & $N$ & $>2,000$ \\
\hline LH1101C & 200 & 300 & N & N & $N$ & 70 & 100 & 700 & $N$ & $>2,000$ \\
\hline LH1102C & 100 & 100 & $<200$ & N & $N$ & 700 & 200 & 500 & N & $>2,000$ \\
\hline LH1103C & 200 & 150 & $<200$ & N & 1,000 & 30 & 100 & 700 & $N$ & $>2,000$ \\
\hline LH1104C & $>200$ & 70 & $<200$ & N & $N$ & 100 & 70 & 700 & $N$ & $>2,000$ \\
\hline LH1105C & 100 & 300 & 200 & 200 & 1,000 & 100 & $N$ & 700 & $N$ & $>2,000$ \\
\hline LH1106C & N & 200 & 300 & $N$ & $N$ & 70 & $N$ & 50 & 700 & 2,000 \\
\hline LH1107C & 20 & N & 200 & N & $N$ & 150 & 700 & 150 & 700 & 1,500 \\
\hline LH1108C & 30 & 300 & 1,500 & 1,500 & 1,500 & 150 & 500 & 2,000 & $N$ & $>2,000$ \\
\hline LH1109C & 10 & 1,000 & 5,000 & N & N & 70 & 150 & 150 & 1,500 & 2,000 \\
\hline LH1110C & 15 & 200 & 1,000 & N & N & 150 & $N$ & 70 & N & $>2,000$ \\
\hline LH1111C & 100 & 20 & 200 & 1,500 & 2,000 & 300 & 500 & 700 & $N$ & $>2,000$ \\
\hline LH1112C & 150 & 100 & $N$ & 1,000 & 1,500 & 50 & 50 & 700 & $N$ & $>2,000$ \\
\hline LH1113C & 100 & 1,500 & N & 3,000 & 3,000 & 30 & 500 & 1,000 & $N$ & $>2,000$ \\
\hline LH1114C & 70 & 150 & N & $>5,000$ & 5,000 & 30 & 500 & 1,500 & N & $>2,000$ \\
\hline LH $1115 \mathrm{C}$ & 10 & 100 & 300 & N & N & 150 & $N$ & 150 & 700 & $>2,000$ \\
\hline LH1116C & 100 & N & N & 1,000 & 1,000 & 20 & 150 & 700 & $N$ & $>2,000$ \\
\hline LH1117C & 30 & N & $<200$ & N & N & 300 & N & 200 & 700 & $>2,000$ \\
\hline LH1118C & 15 & 50 & N & N & N & 30 & 200 & 700 & 500 & $>2,000$ \\
\hline LH1119C & 30 & 300 & $<200$ & $<200$ & $<1,000$ & 150 & 100 & 500 & $N$ & $>2,000$ \\
\hline LH1120C & 100 & $>2,000$ & $<200$ & 200 & 3,000 & 70 & 2,000 & 700 & N & $>2,000$ \\
\hline LH1121C & 20 & 1,500 & 200 & N & N & 100 & 500 & 700 & N & $>2,000$ \\
\hline LH1122C & 50 & N & 200 & 700 & N & 30 & 500 & 1,500 & N & $>2,000$ \\
\hline LH1123C & 70 & 30 & $N$ & $>5,000$ & 5,000 & 30 & 2,000 & 700 & N & $>2,000$ \\
\hline LH1124C & 200 & 200 & $<200$ & 700 & 2,000 & 30 & 200 & 1,000 & N & $>2,000$ \\
\hline LH1125C & 150 & 500 & $<200$ & 200 & 1,000 & 20 & $N$ & 1,000 & N & $>2,000$ \\
\hline LH1126C & 200 & $>2,000$ & N & 1,000 & 5,000 & 20 & 150 & 1,500 & N & $>2,000$ \\
\hline LH1127C & 200 & $>2,000$ & N & 1,000 & 7,000 & 20 & 200 & 1,500 & $N$ & $>2,000$ \\
\hline LH1128C & 50 & 30 & $\boldsymbol{N}$ & $>5,000$ & 5,000 & 20 & 300 & 3,000 & $\mathbf{N}$ & $>2,000$ \\
\hline LH1129C & 70 & 500 & N & $>5,000$ & 5,000 & $<20$ & 500 & $>5,000$ & N & $>2,000$ \\
\hline LH1130C & 70 & $>2,000$ & $<200$ & 3,000 & 7,000 & 30 & 150 & 3,000 & $N$ & $>2,000$ \\
\hline LH1131C & 150 & $>2,000$ & $<200$ & $<200$ & N & 70 & 200 & 1,500 & N & $>2,000$ \\
\hline LH1132C & 100 & $>2,000$ & $<200$ & 1,000 & 3,000 & 50 & 300 & 1,000 & $N$ & $>2,000$ \\
\hline LH1133C & 150 & 1,000 & 200 & 200 & 1,000 & 50 & 100 & 1,500 & $N$ & $>2,000$ \\
\hline
\end{tabular}




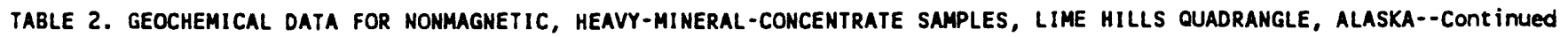

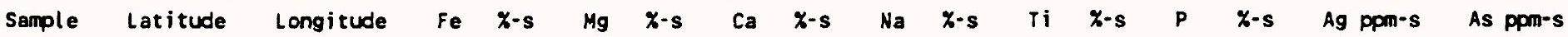

\begin{tabular}{|c|c|c|c|c|c|c|c|c|c|c|c|c|c|}
\hline LH1134C & 6119 & 16 & 154 & & 55 & 3.00 & .10 & 10.00 & .5 & $>2.000$ & 7.0 & 10.0 & 20,000 \\
\hline LH1135C & 6121 & 0 & 154 & 10 & 44 & 1.00 & .30 & 30.00 & .7 & 2.000 & .5 & 5.0 & 3,000 \\
\hline LH1136C & 6120 & 49 & 154 & 11 & 2 & 1.00 & .15 & 50.00 & $<.5$ & 2.000 & $<.5$ & 2.0 & 3,000 \\
\hline LH1137C & 6123 & 43 & 154 & 13 & 37 & 2.00 & .30 & 30.00 & .5 & $>2.000$ & 7.0 & $\mathbf{N}$ & 500 \\
\hline LH1138C & 6123 & 40 & 154 & 13 & 26 & 2.00 & .10 & 20.00 & $<.5$ & $>2.000$ & 7.0 & 3.0 & 3,000 \\
\hline LH1139C & 6127 & 6 & 154 & 7 & 16 & 2.00 & .10 & 20.00 & $<.5$ & $>2.000$ & 3.0 & 5.0 & 20,000 \\
\hline LH1140C & 6129 & 42 & 154 & 103 & 34 & 3.00 & $<.05$ & 3.00 & .5 & $>2.000$ & 1.5 & 7.0 & 3,000 \\
\hline LH1141C & 6127 & 50 & 154 & 12 & 53 & 2.00 & $<.05$ & 30.00 & $<.5$ & $>2.000$ & $<.5$ & 70.0 & $>20,000$ \\
\hline LH1142C & 6126 & 25 & 154 & 10 & 26 & 1.00 & $<.05$ & 20.00 & $<.5$ & $>2.000$ & .7 & 50.0 & 15,000 \\
\hline LH1143C & 6127 & 0 & 154 & 15 & 6 & .50 & $<.05$ & 15.00 & $<.5$ & $>2.000$ & 1.5 & $N$ & 1,000 \\
\hline LH1144C & 6128 & 51 & 154 & 16 & 46 & .70 & $<.05$ & 15.00 & .7 & .700 & .7 & 50.0 & 3,000 \\
\hline LH1145C & 6125 & 8 & 154 & 143 & 34 & .70 & $<.05$ & 15.00 & .5 & 1.000 & .7 & 50.0 & 5,000 \\
\hline LH1146C & 6121 & 23 & 154 & 17 & 17 & 1.00 & .15 & 10.00 & .5 & $>2.000$ & 7.0 & 7.0 & N \\
\hline LH1147C & 6121 & 28 & 154 & 17 & 15 & 1.00 & .20 & 20.00 & $<.5$ & $>2.000$ & 1.5 & 3.0 & N \\
\hline LH1148C & 6121 & 45 & 154 & 21 & 46 & 7.00 & 1.00 & 5.00 & $<.5$ & $>2.000$ & 3.0 & 2.0 & $N$ \\
\hline LH1149C & 6121 & 51 & 154 & 21 & 50 & 1.00 & .30 & 15.00 & .5 & $>2.000$ & 7.0 & $N$ & N \\
\hline LH1150C & 6119 & 38 & 154 & 22 & 46 & 1.00 & .30 & 5.00 & .7 & $>2.000$ & 2.0 & 20.0 & 1.500 \\
\hline LH1151C & 6118 & 57 & 154 & 16 & 10 & 1.00 & .15 & 10.00 & .5 & $>2.000$ & 5.0 & 5.0 & 1,000 \\
\hline LH1152C & 6118 & 28 & 154 & 12 & 29 & 10.00 & .05 & 7.00 & .5 & 2.000 & 1.5 & 50.0 & $>20,000$ \\
\hline LH1153C & 6118 & 31 & 154 & 12 & 25 & 2.00 & .05 & 20.00 & $<.5$ & $>2.000$ & 10.0 & 5.0 & 5,000 \\
\hline LH1154C & 6146 & 48 & 154 & 19 & 21 & 3.00 & 3.00 & 15.00 & .7 & .700 & $N$ & 30.0 & 1,500 \\
\hline LH1155C & 6125 & 28 & 154 & 3 & 17 & 1.00 & .15 & 15.00 & .7 & $>2.000$ & 5.0 & 7.0 & 1,500 \\
\hline LH1156C & 6117 & 38 & 154 & 19 & 58 & 1.00 & .10 & 10.00 & .7 & $>2.000$ & 7.0 & 30.0 & 3,000 \\
\hline LH1157C & 6143 & 43 & 154 & 19 & 38 & 1.50 & 1.50 & 3.00 & $<.5$ & .700 & 1.5 & 10.0 & 3,000 \\
\hline LH1158C & 6140 & 50 & 154 & 16 & 57 & 1.00 & .05 & 3.00 & .5 & 1.000 & 1.0 & $N$ & 2,000 \\
\hline LH1159C & 6139 & 33 & 154 & 12 & 39 & 1.00 & .07 & 10.00 & .5 & .700 & 7.0 & 50.0 & N \\
\hline LH1160C & 6139 & 56 & 154 & 12 & 53 & .70 & $<.05$ & 5.00 & $<.5$ & 1.500 & 3.0 & 50.0 & 700 \\
\hline LH1161C & 6138 & 38 & 154 & 23 & 1 & .50 & .05 & 2.00 & $<.5$ & 2.000 & 1.0 & $N$ & 3,000 \\
\hline LH1162C & 6135 & 49 & 154 & 16 & 28 & 1.50 & $<.05$ & 5.00 & $<.5$ & .200 & 1.5 & 3.0 & 7,000 \\
\hline LH1163C & 6135 & 33 & 154 & 10 & 41 & .50 & .05 & 10.00 & .5 & .300 & 5.0 & N & 500 \\
\hline LH1164C & 6135 & 30 & 154 & 10 & 13 & .70 & $<.05$ & 20.00 & $<.5$ & .150 & 7.0 & $N$ & $N$ \\
\hline LH1165C & 6134 & 1 & 154 & 125 & 57 & .70 & $<.05$ & 1.50 & 1.0 & .150 & 1.5 & 2.0 & 500 \\
\hline LH $1166 \mathrm{C}$ & 6133 & 42 & 154 & 13 & 5 & 2.00 & $<.05$ & 2.00 & $<.5$ & 2.000 & 2.0 & 7.0 & 10,000 \\
\hline LH1167C & 6133 & 9 & 154 & 16 & 5 & 2.00 & .07 & 15.00 & $<.5$ & $>2.000$ & 1.0 & 100.0 & 3,000 \\
\hline LH1168C & 6133 & 9 & 154 & 223 & 39 & 1.00 & .20 & 5.00 & .5 & $>2.000$ & 7.0 & $N$ & N \\
\hline LH1169C & 6130 & 45 & 154 & 15 & 26 & 7.00 & .05 & 10.00 & $<.5$ & $>2.000$ & .7 & N & 5,000 \\
\hline LH1170C & 6130 & 44 & 154 & 22 & 15 & 1.00 & .10 & 20.00 & .5 & $>2.000$ & 1.0 & $N$ & 3,000 \\
\hline LH1171C & 6115 & 11 & 154 & 11 & 8 & 2.00 & .15 & 15.00 & .5 & $>2.000$ & 7.0 & $N$ & N \\
\hline LH1172C & 6113 & 32 & 154 & 123 & 34 & 2.00 & .15 & 30.00 & .5 & $>2.000$ & 20.0 & 20.0 & 2,000 \\
\hline LH1173C & 6111 & 27 & 154 & 13 & 2 & 5.00 & .30 & 1.50 & .5 & $>2.000$ & 7.0 & N & 700 \\
\hline LH1174C & 6110 & 0 & 154 & 20 & 14 & 1.50 & .70 & 5.00 & $<.5$ & $>2.000$ & 7.0 & $N$ & $N$ \\
\hline LH1175C & 619 & 25 & 154 & 14 & 51 & 2.00 & .30 & 3.00 & .5 & $>2.000$ & 5.0 & $\mathbf{N}$ & 700 \\
\hline LH1176C & 618 & 2 & 154 & 8 & 24 & 10.00 & 2.00 & 1.50 & .7 & $>2.000$ & 1.5 & $N$ & 500 \\
\hline LH1177C & 617 & 34 & 154 & 10 & 26 & 7.00 & 1.50 & 10.00 & .7 & $>2.000$ & 5.0 & $\mathbf{N}$ & $<500$ \\
\hline LH1178C & 617 & 37 & 154 & 11 & 54 & 10.00 & 1.50 & 5.00 & .7 & $>2.000$ & 2.0 & N & $<500$ \\
\hline LH1179C & 618 & 1 & 154 & 153 & 38 & 5.00 & .70 & 5.00 & .5 & $>2.000$ & 3.0 & 70.0 & 500 \\
\hline LH1180C & 616 & 40 & 154 & 16 & 9 & 5.00 & .70 & 10.00 & 1.0 & $>2.000$ & 5.0 & 50.0 & $<500$ \\
\hline LH1181C & 617 & 6 & 154 & 3 & 55 & 7.00 & .70 & 1.50 & .5 & $>2.000$ & 3.0 & $N$ & $<500$ \\
\hline LH1182C & 6111 & 20 & 154 & 5 & 49 & 5.00 & .50 & 20.00 & .7 & $>2.000$ & 7.0 & $N$ & $N$ \\
\hline LH1183C & 6113 & 17 & 154 & 6 & 6 & 1.50 & .10 & 30.00 & $<.5$ & $>2.000$ & 20.0 & 15.0 & 700 \\
\hline
\end{tabular}


TABLE 2. GEOCHEMICAL DATA FOR NONMAGNETIC, hEAVY-MIHERAL-CONCENTRATE SAMPLES, LIME hILLS QUAdRANGLE, ALASKa--COntinUed

Sample AU ppm-s B ppm-s Ba ppm-s Be ppm-s Bi ppm-s Cd ppm-s Co ppm-s Cr ppm-s Cu ppm-s

\begin{tabular}{|c|c|c|c|c|c|c|c|c|c|}
\hline LH1134C & $N$ & 300 & 70 & 2 & 100 & $N$ & 700 & 20 & 150 \\
\hline LH1135C & $N$ & 500 & $<50$ & 20 & 150 & $\mathbf{N}$ & $N$ & 70 & 100 \\
\hline LH1136C & $N$ & 100 & $<50$ & 70 & $N$ & $\mathbf{N}$ & N & $<20$ & 10 \\
\hline LH1137C & $N$ & 200 & 70 & 10 & $M$ & $N$ & 20 & 50 & 10 \\
\hline LH $1138 \mathrm{C}$ & $N$ & 500 & $<50$ & 5 & 30 & $N$ & 100 & $<20$ & 200 \\
\hline LK1139C & N & 500 & 50 & 15 & 200 & $N$ & 700 & 30 & 70 \\
\hline LH1140C & $N$ & $N$ & $>10,000$ & 3 & 300 & $N$ & 70 & $<20$ & 70 \\
\hline LH1141C & $N$ & $N$ & 100 & 30 & 200 & $N$ & 200 & 20 & 10 \\
\hline LH1142C & $\mathbf{N}$ & $N$ & 70 & 500 & $N$ & $\mathbf{N}$ & 100 & $<20$ & 100 \\
\hline LH1143C & $N$ & 20 & 70 & 7 & $N$ & $N$ & $N$ & $<20$ & 10 \\
\hline LH1144C & $\mathbf{N}$ & N & $<50$ & 5 & $N$ & $N$ & 50 & $<20$ & 10 \\
\hline LH1145C & $N$ & $N$ & 50 & 7 & $N$ & $N$ & 50 & $<20$ & 30 \\
\hline LH1146C & $N$ & 20 & 200 & 3 & 150 & $N$ & 20 & 70 & 10 \\
\hline LH1147C & $N$ & 20 & 1,000 & 2 & 30 & $\mathbf{N}$ & 20 & 70 & 30 \\
\hline LH1148C & $N$ & 70 & 300 & 3 & 700 & $N$ & 30 & 200 & 100 \\
\hline LH1149C & $N$ & 500 & 700 & 5 & 200 & $N$ & N & 100 & 50 \\
\hline LH1150C & $N$ & 700 & 500 & 3 & 200 & $N$ & 30 & 100 & 50 \\
\hline LH1151C & $N$ & 100 & 200 & 3 & $N$ & $N$ & $N$ & 100 & 50 \\
\hline LH1152C & N & N & 70 & 15 & 300 & $N$ & 1,000 & $<20$ & 150 \\
\hline LH1153C & $N$ & 70 & 100 & 3 & 150 & $N$ & 150 & $<20$ & 100 \\
\hline LH $1154 \mathrm{C}$ & N & 300 & $>10,000$ & 7 & $<20$ & $N$ & 30 & 70 & 50 \\
\hline LH1155C & $N$ & 300 & 300 & 7 & 70 & $N$ & 100 & 20 & 70 \\
\hline LH1156C & N & 700 & 150 & 3 & 150 & $N$ & 100 & $<20$ & $<10$ \\
\hline LH $1157 \mathrm{C}$ & $N$ & 700 & 7,000 & $\mathbf{N}$ & N & $N$ & 30 & 200 & 30 \\
\hline LH1158C & N & N & 200 & 3 & 150 & N & 150 & $<20$ & 10 \\
\hline LH1159C & N & N & 300 & 3 & 70 & $\mathbf{N}$ & $<20$ & $<20$ & 100 \\
\hline LH1160C & $N$ & N & 150 & 5 & 500 & $N$ & 50 & $<20$ & 100 \\
\hline LH1161C & $N$ & N & 150 & 2 & $N$ & $N$ & $N$ & 20 & 50 \\
\hline LH1162C & $N$ & $N$ & 700 & 20 & $N$ & $N$ & 200 & $<20$ & 30 \\
\hline LH1163C & $N$ & $N$ & 200 & $N$ & N & $N$ & 30 & $<20$ & 50 \\
\hline LH1164C & $N$ & $N$ & 100 & $N$ & N & N & 50 & $<20$ & 20 \\
\hline LH1165C & $N$ & $\mathbf{N}$ & 300 & N & N & N & 70 & $<20$ & 500 \\
\hline LH1166C & $N$ & $N$ & 300 & 3 & $N$ & N & 100 & $<20$ & 50 \\
\hline LH1167C & $N$ & 20 & 300 & 20 & $N$ & N & 100 & 30 & 100 \\
\hline LH1168C & $N$ & $N$ & 700 & $N$ & $N$ & $N$ & $N$ & 100 & 15 \\
\hline LH1169C & $N$ & 20 & 70 & 20 & 300 & $N$ & 50 & 20 & 10 \\
\hline LH1170C & N & $N$ & 1,500 & 10 & $N$ & $N$ & 50 & 50 & 10 \\
\hline LH $1171 \mathrm{C}$ & $N$ & 500 & 150 & 5 & 50 & $N$ & 20 & 30 & 50 \\
\hline LH1172C & N & 150 & 150 & 2 & 150 & $\mathbf{N}$ & 70 & 20 & 150 \\
\hline LH1173C & N & $N$ & $>10,000$ & $N$ & $N$ & H & 20 & 150 & 70 \\
\hline LH1174C & $N$ & 500 & 7.000 & 2 & $N$ & $N$ & $<20$ & 200 & 10 \\
\hline LH1175C & N & 20 & 5,000 & $N$ & N & $N$ & 30 & 200 & 20 \\
\hline LH1176C & $\mathbf{N}$ & 30 & $>10,000$ & 2 & N & $N$ & 50 & 150 & 200 \\
\hline LH1177C & $N$ & 100 & $>10,000$ & $N$ & $N$ & $N$ & 50 & 200 & 200 \\
\hline LH1178C & $N$ & 70 & 2,000 & 2 & $N$ & $N$ & 70 & 150 & 100 \\
\hline LH1179C & 300 & 20 & 1,500 & N & $\mathbf{N}$ & $N$ & 30 & 150 & 70 \\
\hline LH1180C & 100 & 50 & 2,000 & 300 & $N$ & $N$ & 50 & 150 & 70 \\
\hline LH1181C & $N$ & 20 & $>10,000$ & 2 & $N$ & N & 50 & 150 & 70 \\
\hline LH1182C & N & 100 & 500 & 3 & N & $N$ & $<20$ & 50 & 20 \\
\hline LH1183C & $\mathbf{N}$ & 30 & 150 & 2 & $<20$ & $N$ & 20 & 20 & 10 \\
\hline
\end{tabular}


TABLE 2. GEOCHEMICAL DATA FOR NONMAGNETIC, heAVY-MINERAL-CONCENTRATE SAMPLES, LIME hILLS QUAdRANGLE, ALASKA--CONTInUEd

Sample Ga ppm-s Ge ppm-s La ppm-s Mn ppm-s Mo ppm-s Nb ppm-s Ni ppm-s Pb ppm-s Pt ppm-s Sb ppm-s

\begin{tabular}{|c|c|c|c|c|c|c|c|c|c|}
\hline LH1134C & $<10$ & $N$ & $>2,000$ & 5,000 & N & 100 & 1,000 & 100 & N \\
\hline LH1135C & $<10$ & $N$ & $>2,000$ & 700 & 100 & 300 & 70 & 300 & $\mathbf{N}$ \\
\hline LH1136C & $<10$ & $N$ & 1,500 & 500 & N & 300 & 15 & 150 & N \\
\hline LH1137C & 15 & $N$ & $>2,000$ & 3,000 & $N$ & 300 & 15 & 100 & N \\
\hline LH1138C & $<10$ & $N$ & $>2,000$ & 7,000 & $N$ & 100 & 100 & 50 & N \\
\hline LH1139C & $<10$ & N & $>2,000$ & 2,000 & 30 & 100 & 700 & 500 & N \\
\hline LH1140C & $<10$ & $N$ & 200 & 200 & $\mathbf{N}$ & 70 & 50 & 150 & N \\
\hline LH1141C & $N$ & $N$ & 1,500 & 200 & $N$ & 500 & 150 & 700 & N \\
\hline LH1142C & $<10$ & $N$ & 1,500 & 300 & 30 & 1,000 & 50 & 5,000 & $N$ \\
\hline LH1143C & $<10$ & $N$ & 1,000 & 700 & 20 & 700 & $N$ & 700 & $N$ \\
\hline LH1144C & $<10$ & $N$ & 700 & 150 & 30 & 70 & 50 & 1,500 & $N$ \\
\hline LH1145C & $N$ & $N$ & 700 & 150 & 30 & 70 & 50 & 700 & $N$ \\
\hline LH1146C & $<10$ & $N$ & 200 & 3,000 & N & 200 & 10 & 300 & $N$ \\
\hline LH1147C & $<10$ & $N$ & 150 & 700 & 100 & 150 & 20 & 700 & $N$ \\
\hline LH1148C & $<10$ & $N$ & 150 & 500 & N & 200 & 100 & 50 & N \\
\hline LK1149C & $<10$ & N & 300 & 1,500 & N & 150 & 30 & 20 & N \\
\hline LK1150C & $<10$ & $\mathbf{N}$ & N & 500 & $N$ & 150 & 50 & 200 & N \\
\hline LH1151C & $<10$ & $N$ & 150 & 2,000 & 50 & 300 & 20 & 15,000 & N \\
\hline LH1152C & $<10$ & $N$ & 200 & 700 & $N$ & 200 & 700 & 500 & N \\
\hline LH1153C & $<10$ & $N$ & 700 & 5,000 & N & 150 & 100 & 300 & N \\
\hline LH1154C & $<10$ & $N$ & 100 & 500 & $N$ & $<50$ & 30 & 2,000 & N \\
\hline LH1155C & 15 & $N$ & 1,500 & 3,000 & 20 & 500 & 70 & 700 & N \\
\hline LH1156C & $<10$ & $N$ & 300 & 3,000 & $N$ & 100 & 50 & 300 & $N$ \\
\hline LH1157C & 20 & $N$ & N & 500 & N & $<50$ & 70 & 1,000 & N \\
\hline LH1158C & $<10$ & $<20$ & 300 & 100 & N & $<50$ & 70 & 100 & N \\
\hline LH1159C & $<10$ & $N$ & 500 & 300 & 30 & $<50$ & $<10$ & 500 & N \\
\hline LH1160C & $<10$ & N & 300 & 300 & 1,000 & $<50$ & 20 & 7,000 & $N$ \\
\hline LH1161C & $<10$ & N & 150 & 300 & N & $<50$ & $<10$ & 100 & $N$ \\
\hline LH1162C & 15 & N & 200 & 300 & $N$ & $<50$ & 100 & 500 & N \\
\hline LH1163C & $<10$ & $N$ & 300 & 200 & N & $<50$ & 20 & 30 & N \\
\hline LH1164C & $<10$ & $N$ & 1,000 & 500 & N & $N$ & 10 & 20 & N \\
\hline LH1165C & $<10$ & $N$ & 500 & 100 & N & $N$ & 50 & 200 & N \\
\hline LH1166C & $<10$ & N & 300 & 300 & N & 50 & 100 & 150 & N \\
\hline LH1167C & 10 & $N$ & 1,500 & 500 & $N$ & 200 & 70 & 70 & $N$ \\
\hline LH1168C & $<10$ & $N$ & 300 & 1,000 & N & 50 & 15 & 20 & $N$ \\
\hline LH1169C & $<10$ & N & 700 & 200 & $N$ & 300 & 20 & 700 & N \\
\hline LH1170C & $<10$ & $<20$ & 1,500 & 300 & $N$ & 500 & 20 & 100 & $N$ \\
\hline LH1171C & 10 & $N$ & 700 & 3,000 & $N$ & 1,000 & 10 & 30 & $N$ \\
\hline LH1172C & 10 & N & 700 & 10,000 & $N$ & 200 & 70 & 15 & $N$ \\
\hline LH1173C & $<10$ & N & 500 & 500 & $N$ & 200 & 30 & 50 & $N$ \\
\hline LH1174C & 15 & N & 1,000 & 2,000 & $N$ & 50 & 10 & 20 & N \\
\hline LH1175C & $<10$ & $N$ & 150 & 200 & N & 200 & 30 & 20 & N \\
\hline LH1176C & 10 & N & 700 & 2,000 & $N$ & 100 & 100 & 70 & N \\
\hline LH1177C & 10 & $N$ & 500 & 3,000 & $N$ & 100 & 100 & 50 & N \\
\hline LH1178C & 20 & $N$ & $>2,000$ & 3,000 & N & 150 & 70 & 50 & $N$ \\
\hline LH1179C & 10 & $N$ & 700 & 1,500 & N & 150 & 30 & 50 & N \\
\hline LH1180C & 20 & $N$ & 150 & 1,500 & $N$ & 70 & 30 & 30 & N \\
\hline LH1181C & 10 & $N$ & 1,000 & 1,500 & $N$ & 150 & 70 & 30 & N \\
\hline LH1182C & 15 & $\mathbf{N}$ & 700 & 5,000 & $N$ & 150 & 10 & 20 & $N$ \\
\hline LH1183C & $<10$ & $N$ & 700 & 7,000 & N & 200 & 20 & 50 & N \\
\hline
\end{tabular}

20,000 
TABLE 2. GEOCHEMICAL DATA FOR NONMAGNETIC, HEAVY-MINERAL-CONCENTRATE SAMPLES, LIME hILLS QUADRANGLE, ALASKA--CONT InUEd

Sample Sc ppm-s Sn ppm-s Sr ppm-s Th ppm-s $U$ ppm-s $V$ ppm-s $\quad W$ ppm-s $\quad Y$ ppm-s Zn ppm-s Zr ppm-s

\begin{tabular}{|c|c|c|c|c|c|c|c|c|c|c|}
\hline LH1134C & 200 & $>2,000$ & $<200$ & 500 & 2,000 & 20 & 200 & 1,500 & N & $>2,000$ \\
\hline LH1135C & 150 & 1,500 & $<200$ & $>5,000$ & 3,000 & 70 & $\mathbf{N}$ & $>5,000$ & $N$ & $>2,000$ \\
\hline LH1136C & 100 & 2,000 & 200 & $>5,000$ & 3,000 & $N$ & $N$ & $>5,000$ & $N$ & $>2,000$ \\
\hline LH1137C & 200 & 700 & $<200$ & 3,000 & 1,500 & 50 & 150 & 5,000 & $N$ & $>2,000$ \\
\hline LH1138C & 200 & 1,500 & $<200$ & 500 & $<1,000$ & 30 & 70 & 3,000 & N & $>2,000$ \\
\hline LH1139C & 200 & 700 & $<200$ & 3,000 & 7,000 & 30 & 200 & $>5,000$ & N & $>2,000$ \\
\hline LH1140C & 70 & 50 & $<200$ & 5,000 & 3,000 & 50 & 1,500 & 700 & N & $>2,000$ \\
\hline LH1141C & 150 & 1,500 & $<200$ & $>5,000$ & 3,000 & 50 & 100 & $>5,000$ & $\mathbf{N}$ & $>2,000$ \\
\hline LH1142C & $>200$ & 150 & $<200$ & $>5,000$ & 5,000 & 70 & $N$ & $>5,000$ & N & $>2,000$ \\
\hline LH1143C & 150 & $>2,000$ & $N$ & $>5,000$ & 5,000 & 50 & 150 & 5,000 & N & $>2,000$ \\
\hline LH1144C & 70 & 300 & N & $>5,000$ & 7,000 & 20 & 1,000 & 3,000 & $N$ & $>2,000$ \\
\hline LH1145C & 70 & 150 & $N$ & $>5,000$ & 7,000 & 20 & 500 & 3,000 & N & $>2,000$ \\
\hline LH1146C & 200 & 700 & 200 & N & $N$ & 200 & 700 & 500 & N & $>2,000$ \\
\hline LH1147C & 100 & 700 & 500 & $N$ & $N$ & 100 & 2,000 & 300 & N & $>2,000$ \\
\hline LH1148C & 150 & $>2,000$ & 500 & $\mathbf{N}$ & N & 300 & 150 & 300 & N & $>2,000$ \\
\hline LH1149C & 150 & $>2,000$ & 700 & N & N & 200 & 100 & 500 & N & $>2,000$ \\
\hline LH1150C & 70 & $>2,000$ & 200 & N & N & 150 & 1,000 & 150 & N & 1,500 \\
\hline LH1151C & 150 & $>2,000$ & 300 & N & $N$ & 150 & 150 & 500 & N & $>2,000$ \\
\hline LH1152C & 50 & $>2,000$ & $N$ & N & 2,000 & 20 & 150 & 300 & 700 & $>2,000$ \\
\hline LH1153C & 150 & $>2,000$ & $<200$ & N & $N$ & 30 & 200 & 700 & N & $>2,000$ \\
\hline LH1154C & 15 & 2,000 & 5,000 & N & $N$ & 150 & 500 & 50 & 1,000 & 1,500 \\
\hline LH1155C & 200 & $>2,000$ & $<200$ & 200 & 1,000 & 50 & 70 & 1,000 & N & $>2,000$ \\
\hline LH1156C & 150 & $>2,000$ & N & $N$ & N & 30 & 200 & 700 & N & $>2,000$ \\
\hline LH1157C & 30 & $>2,000$ & 200 & 500 & N & 500 & 150 & 200 & N & $>2,000$ \\
\hline LH1158C & 50 & 1,000 & N & $>5,000$ & 7,000 & 20 & 300 & 2,000 & $N$ & $>2,000$ \\
\hline LH1159C & 50 & 100 & $<200$ & 500 & N & 30 & 2,000 & 700 & $N$ & $>2,000$ \\
\hline LH1160C & 70 & 700 & $\mathbf{N}$ & 2,000 & 2,000 & 30 & 200 & 1,000 & $\mathbf{N}$ & $>2,000$ \\
\hline LH1161C & 50 & 300 & N & $>5,000$ & 7,000 & 50 & 200 & 1,000 & N & $>2,000$ \\
\hline LH1162C & 30 & 1,000 & $N$ & $>5,000$ & 15,000 & 50 & 500 & 1,500 & N & $>2,000$ \\
\hline LH1163C & 30 & 300 & $<200$ & 500 & N & $<20$ & 300 & 700 & N & $>2,000$ \\
\hline LH1164C & 70 & 30 & $<200$ & 500 & N & $\mathbf{N}$ & 50 & 700 & N & $>2,000$ \\
\hline LH1165C & 30 & 2,000 & $N$ & 2,000 & 2,000 & $N$ & 100 & 500 & N & $>2,000$ \\
\hline LH1166C & 50 & 700 & $N$ & $>5,000$ & 3,000 & 20 & 100 & 700 & H & $>2,000$ \\
\hline LH1167C & 70 & 200 & N & $>5,000$ & 3,000 & 100 & 300 & 1,500 & N & $>2,000$ \\
\hline LH1168C & 70 & 150 & 500 & 200 & $N$ & 150 & 100 & 500 & $N$ & $>2,000$ \\
\hline LH1169C & 100 & 500 & $N$ & $>5,000$ & 3,000 & 70 & 150 & 5,000 & N & $>2,000$ \\
\hline LH1170C & 100 & 150 & $<200$ & $>5,000$ & 5,000 & 150 & 150 & $>5,000$ & N & $>2,000$ \\
\hline LH1171C & 200 & $>2,000$ & 200 & N & N & 70 & 150 & 700 & N & $>2,000$ \\
\hline LH1172C & 100 & 1,000 & $<200$ & N & N & 70 & 100 & 1,000 & $N$ & $>2,000$ \\
\hline LH1173C & 150 & 1,500 & 3,000 & N & N & 300 & $N$ & 200 & $N$ & $>2,000$ \\
\hline LH1174C & 100 & $>2,000$ & 500 & $N$ & N & 150 & 150 & 500 & $N$ & $>2,000$ \\
\hline LH1175C & 30 & 300 & 1,000 & $N$ & N & 500 & 100 & 150 & $N$ & $>2,000$ \\
\hline LH1176C & 150 & 1,000 & 500 & N & N & 300 & $N$ & 150 & N & $>2,000$ \\
\hline LH1177C & 50 & 20 & 1,500 & N & $N$ & 300 & N & 150 & N & $>2,000$ \\
\hline LH $1178 \mathrm{C}$ & 100 & 20 & 700 & $\mathbf{N}$ & N & 500 & N & 300 & $N$ & $>2,000$ \\
\hline Lн1179С & 50 & 1,500 & 700 & N & N & 500 & $N$ & 200 & $N$ & $>2,000$ \\
\hline LH1180C & 30 & $>2,000$ & 700 & $N$ & $N$ & 300 & N & 300 & $\mathbf{N}$ & $>2,000$ \\
\hline LH1181C & 100 & 1,500 & 1,000 & N & N & 500 & N & 200 & N & $>2,000$ \\
\hline LH1182C & 70 & 700 & $<200$ & $N$ & $N$ & 150 & 100 & 700 & N & $>2,000$ \\
\hline LH1183C & 100 & 500 & $<200$ & $N$ & $N$ & 70 & 50 & 1,000 & N & $>2,000$ \\
\hline
\end{tabular}




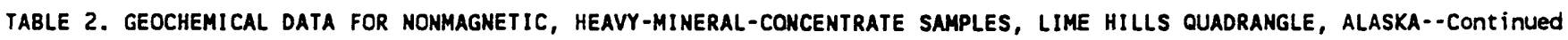

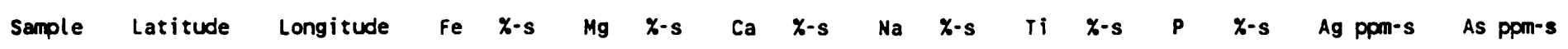

\begin{tabular}{|c|c|c|c|c|c|c|c|c|c|c|c|}
\hline LH1184C & 60 & 5955 & 153415 & 50 & .70 & .30 & 30.00 & 1.0 & $>2.000$ & 15.0 & N \\
\hline LH1185C & 61 & 039 & 15348 & 0 & 5.00 & 1.50 & 30.00 & .7 & 2.000 & 3.0 & N \\
\hline LH1186C & 61 & 031 & 15348 & 3 & 1.00 & 1.00 & 20.00 & 2.0 & 2.000 & 3.0 & N \\
\hline LH1187C & 61 & 226 & 153444 & 42 & 5.00 & 1.50 & 20.00 & .5 & $>2.000$ & 3.0 & N \\
\hline LH1188C & 61 & 222 & 153445 & 53 & 1.50 & .70 & 30.00 & 1.5 & $>2.000$ & 7.0 & N \\
\hline LH1189C & 61 & 233 & 153455 & 58 & 3.00 & 1.00 & 30.00 & 1.0 & $>2.000$ & 7.0 & N \\
\hline LH1190C & 61 & 357 & 153464 & 47 & 7.00 & 1.50 & 20.00 & .7 & $>2.000$ & 2.0 & N \\
\hline LH1191C & 61 & 745 & 15340 & 8 & 1.00 & 5.00 & 30.00 & 1.0 & $>2.000$ & 5.0 & N \\
\hline LH1192C & 61 & 85 & 15339 & 6 & 2.00 & 3.00 & 20.00 & 1.0 & $>2.000$ & 3.0 & N \\
\hline LH1193C & 61 & 037 & 15338 & 9 & 2.00 & 1.00 & 7.00 & .5 & $>2.000$ & 1.5 & N \\
\hline LH1194C & 61 & 114 & 153393 & 32 & 2.00 & .30 & 10.00 & .5 & $>2.000$ & 7.0 & $N$ \\
\hline LH1195C & 61 & 147 & 153393 & 30 & 1.00 & .50 & 20.00 & .5 & $>2.000$ & 7.0 & N \\
\hline LH1196C & 61 & 157 & 153401 & 15 & 1.00 & .20 & 10.00 & 1.5 & $>2.000$ & 1.5 & N \\
\hline LH1197C & 61 & 53 & 153404 & 42 & 15.00 & .50 & 7.00 & .7 & 2.000 & 5.0 & N \\
\hline LH1198C & 61 & 459 & 153403 & 32 & 1.00 & .15 & 7.00 & 1.5 & $>2.000$ & 5.0 & N \\
\hline 내1199C & 61 & 510 & 153401 & 16 & 7.00 & .50 & 20.00 & 1.5 & 1.500 & 5.0 & N \\
\hline LH1200C & 61 & 514 & 153404 & 40 & 2.00 & .70 & 20.00 & 1.5 & 2.000 & 7.0 & 50.0 \\
\hline LH1201C & 61 & 544 & 153401 & 13 & 3.00 & .70 & 30.00 & .7 & $>2.000$ & 7.0 & N \\
\hline LH1202C & 61 & 1418 & 15328 & 4 & 50.00 & .10 & .70 & .7 & .500 & N & 2.0 \\
\hline LH1203C & 61 & 1035 & 153295 & 58 & 5.00 & .15 & 5.00 & .7 & $>2.000$ & 2.0 & $\mathbf{N}$ \\
\hline LH1204C & 615 & 553 & 154465 & 51 & 7.00 & 3.00 & 10.00 & 1.0 & $>2.000$ & .5 & N \\
\hline LH $1205 \mathrm{C}$ & 615 & 5333 & 154464 & 48 & 1.50 & .50 & 1.50 & $<.5$ & .300 & $N$ & N \\
\hline LH1206C & 615 & 537 & 154475 & 52 & 10.00 & 3.00 & 10.00 & 1.0 & $>2.000$ & .7 & N \\
\hline LH1207C & 615 & 5218 & 154472 & 21 & 1.00 & .20 & 1.50 & .5 & .150 & .5 & N \\
\hline LH1208C & 615 & 5146 & 154493 & 33 & 7.00 & 3.00 & 20.00 & 1.5 & $>2.000$ & .5 & N \\
\hline LH1209C & 61 & 5018 & 154471 & 12 & .70 & .15 & 1.50 & $<.5$ & .200 & .7 & $N$ \\
\hline LH1210C & 61 & 4953 & 154484 & 40 & 7.00 & 5.00 & 15.00 & 1.5 & $>2.000$ & .5 & N \\
\hline LH1211C & 61 & 4655 & 154484 & 42 & 1.00 & .50 & 2.00 & $<.5$ & .500 & $<.5$ & N \\
\hline LH1212C & 61 & 4556 & 15451 & 7 & 2.00 & 1.00 & 5.00 & 1.0 & $>2.000$ & 1.5 & N \\
\hline LH1213C & 61 & 4729 & 154443 & 34 & 1.00 & 1.00 & 10.00 & .5 & $>2.000$ & 3.0 & N \\
\hline LH1214C & 615 & 5057 & 154442 & 26 & 1.00 & .50 & 1.50 & $<.5$ & .700 & .7 & $N$ \\
\hline LH1215C & 61 & 445 & 153293 & 36 & 2.00 & .20 & 1.50 & 1.5 & 2.000 & .5 & 2.0 \\
\hline LH1216C & 61 & 525 & 153293 & 39 & 3.00 & 1.00 & 7.00 & .5 & $>2.000$ & 1.5 & 5.0 \\
\hline LH1217C & 61 & 335 & 15334 & 9 & 5.00 & 5.00 & 10.00 & 1.0 & $>2.000$ & 1.5 & $N$ \\
\hline LH1218C & 61 & 219 & 153343 & 30 & 5.00 & 7.00 & 20.00 & $<.5$ & 1.500 & $<.5$ & 3.0 \\
\hline LH1219C & 61 & 25 & 153304 & 45 & 1.00 & .50 & 10.00 & 1.0 & $>2.000$ & 1.5 & N \\
\hline LH1220C & 61 & 014 & 153323 & 32 & 7.00 & .15 & 3.00 & .7 & $>2.000$ & 1.0 & 200.0 \\
\hline LH1221C & 61 & 112 & 15328 & 1 & 2.00 & 1.00 & 20.00 & 1.0 & $>2.000$ & 3.0 & N \\
\hline LH1222C & 61 & 050 & 153301 & 17 & 2.00 & .20 & 7.00 & 1.0 & $>2.000$ & 2.0 & 10.0 \\
\hline LH1223C & 61 & 248 & 153222 & 22 & 7.00 & 5.00 & 10.00 & 1.0 & 2.000 & 2.0 & 7.0 \\
\hline LH $1224 \mathrm{C}$ & 61 & 13 & 153204 & 43 & 5.00 & .50 & 7.00 & 1.5 & $>2.000$ & 2.0 & 20.0 \\
\hline LH $1225 \mathrm{C}$ & 61 & 10 & 153195 & 51 & 5.00 & .15 & 15.00 & $<.5$ & $>2.000$ & 7.0 & 7.0 \\
\hline LH1226C & 61 & 024 & 153155 & 57 & .50 & .05 & 1.00 & 1.0 & 1.500 & 1.5 & N \\
\hline LH1227C & 61 & 220 & 153154 & 42 & 3.00 & .50 & 1.00 & 1.0 & 2.000 & 1.5 & 70.0 \\
\hline LH1228C & 61 & 459 & 153204 & 48 & 15.00 & 1.00 & 10.00 & $<.5$ & 2.000 & 5.0 & 30.0 \\
\hline LH1229C & 61 & 526 & 153233 & 33 & 15.00 & .50 & 20.00 & $<.5$ & 1.500 & 7.0 & 2.0 \\
\hline LH $1230 \mathrm{C}$ & 61 & 72 & 153212 & 22 & 3.00 & 10.00 & 20.00 & $<.5$ & .500 & N & 20.0 \\
\hline LH1231C & 61 & 651 & 153215 & 56 & 3.00 & .05 & 1.50 & $\mathbf{N}$ & .200 & .7 & 5.0 \\
\hline LH1232C & 614 & 4615 & 15544 & 43 & 7.00 & 3.00 & 15.00 & .7 & $>2.000$ & .5 & $\mathbf{N}$ \\
\hline LH1233C & 614 & 4657 & 154552 & 28 & 5.00 & 1.50 & 20.00 & .7 & $>2.000$ & $\mathbf{N}$ & $\mathbf{N}$ \\
\hline
\end{tabular}


TABLE 2. GEOCHEMICAL DATA FOR NONMAGNETIC, hEAVY-MINERAL-CONCENTRATE SAMPLES, LIME hILlS QUADRANGLE, ALASKa--ContinUed

Sample AU ppm-s B ppm-s Ba ppm-s Be ppm-s Bi ppm-s Co Copm-s Co ppm-s Cr ppm-s CU ppm-s

\begin{tabular}{|c|c|c|c|c|c|c|c|c|c|}
\hline LH1184C & $\mathbf{N}$ & N & 150 & $\mathbf{N}$ & N & $N$ & $<20$ & 20 & 10 \\
\hline LH1185C & N & 50 & 1,500 & 2 & N & N & 70 & 70 & 70 \\
\hline LH1186C & $N$ & 20 & 300 & N & $N$ & N & 50 & 70 & 15 \\
\hline LH1187C & $\mathbf{N}$ & 20 & 1,000 & N & $\mathbf{N}$ & $\mathbf{N}$ & 50 & 100 & 70 \\
\hline LH1188C & $\mathbf{N}$ & 1,500 & 300 & N & $\mathbf{N}$ & $\mathbf{N}$ & 20 & 70 & 30 \\
\hline LH1189C & $N$ & 100 & 150 & N & $\mathbf{N}$ & N & 20 & 70 & 30 \\
\hline LH1190C & $\mathbf{N}$ & 100 & 300 & 2 & N & $\mathbf{N}$ & 100 & 50 & 150 \\
\hline LH1191C & N & N & 200 & $N$ & N & N & $<20$ & 20 & 10 \\
\hline LH1192C & $\mathbf{N}$ & $N$ & 700 & $\mathbf{N}$ & $\mathbf{N}$ & N & 20 & $<20$ & $<10$ \\
\hline LH1193C & N & N & 200 & $\mathbf{N}$ & N & N & 300 & $<20$ & 30 \\
\hline LH1194C & N & N & 200 & $\mathbf{N}$ & N & N & 150 & 20 & 100 \\
\hline LH1195C & $N$ & $N$ & 70 & $N$ & $N$ & $\mathbf{N}$ & 70 & 30 & 20 \\
\hline LK1196C & $N$ & N & 300 & $\mathbf{N}$ & N & $\mathbf{N}$ & 30 & $<20$ & 15 \\
\hline LH1197C & N & 200 & 5,000 & $N$ & $N$ & $N$ & 200 & 30 & 150 \\
\hline LH1198C & $\mathbf{N}$ & $N$ & 700 & N & $\mathbf{N}$ & $\mathbf{N}$ & 100 & $<20$ & 10 \\
\hline LH1199C & $\mathbf{N}$ & $N$ & 200 & N & $N$ & $\mathbf{N}$ & 300 & $<20$ & 70 \\
\hline LH1200C & 30 & N & 500 & $\mathbf{N}$ & $N$ & N & 30 & 20 & 70 \\
\hline LH1201C & $\mathbf{N}$ & 30 & 1,500 & $\mathbf{N}$ & $N$ & N & 50 & 20 & 70 \\
\hline LH1202C & $\mathbf{N}$ & $N$ & 1,500 & N & $\mathbf{N}$ & N & 200 & $<20$ & 100 \\
\hline LH1203C & $N$ & N & 7,000 & 3 & $\mathbf{N}$ & $\mathbf{N}$ & 20 & $<20$ & $<10$ \\
\hline LH1204C & $N$ & 150 & 3,000 & 3 & $\mathbf{N}$ & N & 50 & 500 & 70 \\
\hline LH1205C & $N$ & N & $>10,000$ & $N$ & N & N & $N$ & 20 & 30 \\
\hline LH1206C & N & 300 & $>10,000$ & 7 & $N$ & N & 50 & 300 & 70 \\
\hline LH1207C & N & 20 & $>10,000$ & $\mathbf{N}$ & $\mathbf{N}$ & N & $N$ & 30 & 10 \\
\hline LH1208C & N & 200 & $>10,000$ & 2 & $N$ & N & 30 & 700 & 70 \\
\hline LH1209C & $\mathbf{N}$ & N & $>10,000$ & $\mathbf{N}$ & N & $\mathbf{N}$ & $\mathbf{N}$ & 20 & 10 \\
\hline LH1210C & $\mathbf{N}$ & 200 & $>10,000$ & 5 & N & $\mathbf{N}$ & 30 & 500 & 100 \\
\hline LH1211C & $\mathbf{N}$ & 100 & $>10,000$ & N & N & $\mathbf{N}$ & N & 30 & 30 \\
\hline LH1212C & N & 500 & $>10,000$ & $\mathbf{N}$ & N & $\mathbf{N}$ & $<20$ & 70 & 50 \\
\hline LH1213C & N & 700 & $>10,000$ & 15 & N & N & N & 200 & $<10$ \\
\hline LH1214C & $\mathbf{N}$ & 20 & $>10,000$ & $\mathbf{N}$ & N & N & $\mathbf{N}$ & 50 & 20 \\
\hline LH1215C & $N$ & $N$ & 10,000 & $N$ & 100 & $N$ & 30 & $<20$ & 100 \\
\hline LH1216C & $\mathbf{N}$ & $N$ & 700 & 3 & $\mathbf{N}$ & N & 20 & 30 & 200 \\
\hline LH1217C & $N$ & $N$ & 1,500 & $N$ & 30 & N & 50 & 50 & 150 \\
\hline LH1218C & $\mathbf{N}$ & 20 & 500 & N & $\mathbf{N}$ & $\mathbf{N}$ & 300 & 30 & 70 \\
\hline LH1219C & $N$ & $N$ & $>10,000$ & N & N & $N$ & 20 & 20 & 50 \\
\hline LH1220C & $\mathbf{N}$ & N & 1,000 & N & 100 & $N$ & 50 & $<20$ & 50 \\
\hline LH1221C & N & N & 7,000 & N & $N$ & N & 30 & 50 & 50 \\
\hline LH1222C & $\mathbf{N}$ & N & 1,500 & 5 & 30 & N & 20 & $<20$ & 10 \\
\hline LH1223C & 30 & 500 & 1,500 & N & N & N & 100 & 1,000 & 100 \\
\hline LH1224C & $\mathbf{N}$ & $\mathbf{N}$ & 7,000 & $\mathbf{N}$ & 500 & $N$ & 50 & $<20$ & 70 \\
\hline LH1225C & $\mathbf{N}$ & N & 3,000 & $\mathbf{N}$ & 30 & $N$ & 30 & 30 & 100 \\
\hline LH1226C & $N$ & $N$ & 700 & N & N & $N$ & $N$ & $<20$ & $<10$ \\
\hline LH1227C & N & N & 5,000 & 2 & 1,500 & $N$ & 20 & 30 & 30 \\
\hline LH1228C & N & 1,500 & 5,000 & N & $N$ & $N$ & 150 & 150 & 500 \\
\hline LH1229C & $\mathbf{N}$ & 100 & 7,000 & N & $\mathbf{N}$ & $N$ & 150 & 70 & 300 \\
\hline LH1230C & 30 & 30 & 300 & N & 30 & $N$ & 70 & 70 & 30 \\
\hline LH1231C & $N$ & $N$ & 1,000 & N & $\mathbf{N}$ & $N$ & 50 & $<20$ & 50 \\
\hline LH1232C & N & 70 & 7,000 & $\mathbf{N}$ & N & N & 50 & 700 & 70 \\
\hline LH1233C & $N$ & 300 & 700 & $N$ & $\mathbf{N}$ & N & 30 & 200 & 70 \\
\hline
\end{tabular}


TABLE 2. GEOCHEMICAL DATA fOR NONMAGNETIC, heAVY-MINERAL-CONCENTRATE SAMPLES, LIME hILLS QUADRANGLE, ALASKA--CONTINUEd

Sample Ga ppm-s Ge ppm-s La ppm-s Mn ppm-s Mo ppm-s Nb ppm-s Ni ppm-s Pb ppm-s Pt ppm-s Sb ppm-s

\begin{tabular}{|c|c|c|c|c|c|c|c|c|c|}
\hline LH1184C & $<10$ & $N$ & 700 & 200 & $N$ & 50 & 30 & $<20$ & N \\
\hline LH1185C & 30 & $N$ & 100 & 500 & N & $<50$ & 100 & 30 & N \\
\hline LH1186C & 30 & $N$ & 150 & 1,500 & $N$ & 50 & 30 & $<20$ & $N$ \\
\hline LH1187C & 20 & $\mathbf{N}$ & 200 & 2,000 & 50 & 70 & 30 & 500 & N \\
\hline LH1188C & 15 & N & 200 & 1,500 & N & 100 & 15 & 100 & N \\
\hline LH1189C & 20 & $\mathbf{N}$ & 200 & 1,500 & N & 100 & 20 & $<20$ & N \\
\hline LH1190C & 20 & N & N & 1,500 & N & 50 & 50 & 20 & N \\
\hline LH1191C & 10 & $N$ & 500 & 150 & N & 70 & $<10$ & N & N \\
\hline LH1192C & 10 & N & 300 & 150 & N & 70 & $<10$ & $<20$ & N \\
\hline LH1193C & $<10$ & N & 200 & 100 & 30 & 50 & 15 & 70 & N \\
\hline LH1194C & $<10$ & N & 500 & 100 & N & $<50$ & 50 & $<20$ & $N$ \\
\hline LH1195C & $<10$ & N & 300 & 100 & $<10$ & $<50$ & 150 & $<20$ & 30 \\
\hline LH1196C & 15 & N & 150 & 70 & N & 50 & $<10$ & $<20$ & N \\
\hline LH1197C & 15 & N & 100 & 200 & 10 & $<50$ & 70 & 150 & N \\
\hline LH1198C & 10 & N & 150 & 70 & N & 50 & 10 & $<20$ & N \\
\hline LH1199C & 15 & N & 200 & 500 & $N$ & $<50$ & 50 & 30 & N \\
\hline LH1200C & 15 & N & 200 & 500 & N & $<50$ & 10 & $<20$ & $N$ \\
\hline LH1201C & 10 & N & 300 & 700 & $N$ & $<50$ & 10 & 30 & N \\
\hline LH1202C & 15 & N & N & 150 & 100 & 150 & 30 & 100 & N \\
\hline LH1203C & 15 & N & 1,500 & 700 & 20 & $<50$ & $<10$ & 200 & $N$ \\
\hline LH1204C & 20 & N & 150 & 1,000 & N & 50 & 70 & 30 & N \\
\hline LH1205C & N & N & N & 150 & N & $<50$ & $<10$ & N & N \\
\hline LH1206C & 20 & N & 150 & 1,500 & N & 70 & 70 & 30 & N \\
\hline LH1207C & N & N & N & 150 & N & $<50$ & 10 & N & $N$ \\
\hline LH1208C & 30 & $N$ & 150 & 1,500 & N & 70 & 70 & 30 & N \\
\hline LH1209C & $N$ & $N$ & N & 100 & N & $<50$ & $<10$ & N & N \\
\hline LH1210C & 30 & $N$ & 150 & 1,500 & N & 50 & 70 & 30 & N \\
\hline LH1211C & $<10$ & $N$ & N & 150 & $N$ & $<50$ & 10 & $\mathbf{N}$ & $N$ \\
\hline LH1212C & $<10$ & $N$ & N & 300 & $N$ & $<50$ & 20 & N & N \\
\hline LH1213C & 10 & N & 150 & 500 & N & 70 & 10 & N & $N$ \\
\hline LH1214C & $<10$ & $N$ & $N$ & 150 & N & $<50$ & 15 & N & N \\
\hline LH1215C & 15 & $N$ & N & 1,500 & N & $<50$ & $<10$ & 150 & N \\
\hline LH1216C & 10 & $N$ & $>2,000$ & 3,000 & 10 & 500 & $<10$ & 300 & $N$ \\
\hline LH1217C & 10 & N & 700 & 2,000 & 150 & 100 & 30 & 30 & N \\
\hline LH1218C & $<10$ & $N$ & N & 500 & 30 & $<50$ & 70 & 500 & N \\
\hline LH1219C & $<10$ & $N$ & 300 & 700 & 10 & 150 & 15 & $<20$ & N \\
\hline LH1220C & 10 & N & 700 & 1,000 & 20 & 70 & $<10$ & 500 & N \\
\hline LH1221C & 10 & N & 700 & 1,500 & $<10$ & 150 & 10 & $<20$ & N \\
\hline LH1222C & 15 & N & 200 & 700 & N & 70 & $<10$ & 100 & N \\
\hline LH1223C & 10 & N & 100 & 1,500 & $N$ & 50 & 150 & 50 & $N$ \\
\hline LH1224C & 10 & $N$ & 1,500 & 1,000 & 10 & 70 & $<10$ & 200 & N \\
\hline LH1225C & $<10$ & N & 1,500 & 1,000 & 100 & 70 & 10 & 100 & N \\
\hline LH1226C & $<10$ & K & 2,000 & 500 & 50 & $<50$ & N & 100 & N \\
\hline LH1227C & 10 & $N$ & $>2,000$ & 1,500 & 200 & 70 & $<10$ & 700 & N \\
\hline LH1228C & 10 & N & 150 & 1,000 & 20 & $<50$ & 150 & 3,000 & N \\
\hline LH1229C & $<10$ & N & 100 & 2,000 & 20 & $<50$ & 100 & 300 & N \\
\hline LH1230C & $<10$ & N & N & 3,000 & 70 & $<50$ & 70 & 300 & $N$ \\
\hline LH1231C & $<10$ & $\mathbf{N}$ & 200 & 300 & 100 & $<50$ & $<10$ & 300 & N \\
\hline LH1232C & 15 & N & 150 & 1,000 & 100 & 70 & 70 & 20 & N \\
\hline LH1233C & 20 & N & N & 700 & $N$ & $<50$ & 50 & 20 & N \\
\hline
\end{tabular}




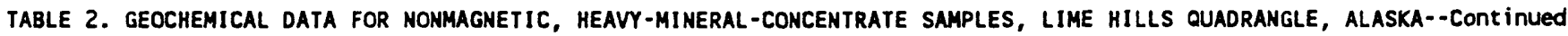
Sample sc ppm-s Sn ppm-s Sr ppm-s Th ppm-s $U$ ppm-s $V$ ppm-s $\quad W$ ppm-s $\quad Y$ ppm-s $2 n$ ppm-s $2 r$ ppm-s

\begin{tabular}{|c|c|c|c|c|c|c|c|c|c|c|}
\hline LH1184C & 15 & N & 1,000 & N & N & 150 & N & 500 & $N$ & $>2,000$ \\
\hline LH1185C & 20 & N & 500 & $<200$ & N & 150 & 300 & 200 & $N$ & $>2,000$ \\
\hline LH1186C & 10 & N & 700 & 500 & N & 150 & 100 & 150 & N & $>2,000$ \\
\hline LH1187C & 20 & $N$ & 700 & N & $N$ & 500 & 100 & 300 & N & $>2,000$ \\
\hline LH1188C & 15 & N & 1,000 & $\mathbf{N}$ & N & 300 & 50 & 300 & N & $>2,000$ \\
\hline LH1189C & 15 & $<20$ & 1,000 & N & N & 500 & N & 300 & N & $>2,000$ \\
\hline LH1190C & 15 & N & 500 & N & N & 300 & 500 & 150 & N & $>2,000$ \\
\hline LH1191C & 30 & 30 & 500 & $<200$ & N & 200 & 200 & 500 & N & $>2,000$ \\
\hline LH1192C & 30 & 50 & 200 & 700 & N & 150 & 1,500 & 500 & N & $>2,000$ \\
\hline LH1193C & 50 & 20 & $<200$ & 200 & N & 300 & 200 & 500 & N & $>2,000$ \\
\hline LH1194C & 70 & $N$ & 500 & 700 & 1,000 & 150 & 300 & 500 & N & $>2,000$ \\
\hline LH1195C & 50 & $N$ & 200 & 700 & N & 150 & 100 & 500 & N & $>2,000$ \\
\hline LH1196C & 15 & $N$ & 500 & 700 & N & 150 & N & 300 & N & $>2,000$ \\
\hline LH1197C & 15 & $N$ & 200 & N & N & 150 & 70 & 200 & $N$ & $>2,000$ \\
\hline LH1198C & 30 & 20 & 300 & 700 & N & 150 & 100 & 300 & N & $>2,000$ \\
\hline LH1199C & 15 & N & 700 & 200 & N & 100 & 50 & 300 & $\mathbf{N}$ & $>2,000$ \\
\hline LH1200C & 10 & N & 700 & N & N & 150 & 70 & 200 & $N$ & $>2,000$ \\
\hline LH1201C & 20 & $\mathbf{N}$ & 500 & N & N & 200 & 100 & 500 & N & $>2,000$ \\
\hline LH1202C & 10 & 20 & N & N & N & 30 & N & 150 & 1.000 & $>2,000$ \\
\hline LH1203C & 100 & 300 & $<200$ & 1,500 & 1,000 & 150 & 300 & 700 & N & $>2,000$ \\
\hline LH1204C & 30 & N & 300 & N & N & 300 & N & 150 & N & $>2,000$ \\
\hline LH1205C & N & $\mathbf{N}$ & 7,000 & $N$ & $N$ & 20 & N & 20 & N & 700 \\
\hline LH1206C & 30 & 50 & 500 & $N$ & N & 200 & $\mathbf{N}$ & 150 & $\mathbf{N}$ & $>2,000$ \\
\hline LH1207C & N & 30 & 5,000 & N & N & 20 & N & 30 & N & 700 \\
\hline LH1208C & 30 & 30 & 700 & N & N & 300 & $N$ & 150 & N & $>2,000$ \\
\hline LH1209C & N & N & 7,000 & N & $\mathbf{N}$ & 30 & $N$ & 50 & N & 2,000 \\
\hline LH1210C & 30 & 200 & 700 & $N$ & N & 200 & N & 200 & N & $>2,000$ \\
\hline LH1211C & N & N & 7,000 & N & N & 50 & N & 30 & 500 & 2,000 \\
\hline LH1212C & 15 & 300 & 1,500 & N & N & 150 & N & 150 & $N$ & $>2,000$ \\
\hline LH1213C & 50 & 1,500 & 700 & 200 & $<1,000$ & 150 & 150 & 500 & $N$ & $>2,000$ \\
\hline LH1214C & $\mathbf{N}$ & 100 & 3,000 & N & $\mathbf{N}$ & 70 & N & 70 & $N$ & $>2,000$ \\
\hline LH1215C & 50 & N & $<200$ & N & N & 30 & 100 & 700 & N & $>2,000$ \\
\hline LH1216C & 200 & 300 & $<200$ & 5,000 & 2,000 & 200 & 150 & 2,000 & N & $>2,000$ \\
\hline LH1217C & 50 & 70 & $<200$ & 500 & $<1,000$ & 150 & 200 & 700 & N & $>2,000$ \\
\hline LH1218C & 10 & N & $<200$ & N & N & 100 & 150 & 150 & $\mathbf{N}$ & $>2,000$ \\
\hline LH1219C & 15 & 50 & 1,000 & 1,000 & N & 150 & 200 & 500 & N & $>2,000$ \\
\hline LH1220C & 100 & N & 300 & 200 & N & 150 & 500 & 500 & N & $>2,000$ \\
\hline LH1221C & 20 & 100 & 200 & 200 & N & 300 & N & 500 & N & $>2,000$ \\
\hline LH1222C & 30 & 20 & 300 & 700 & $<1,000$ & 150 & 200 & 300 & N & $>2,000$ \\
\hline LH1223C & 30 & N & 200 & N & N & 200 & 700 & 150 & N & $>2,000$ \\
\hline LH1224C & 100 & 30 & 200 & 1,000 & 1,000 & 150 & 700 & 700 & $N$ & $>2,000$ \\
\hline LH1225C & 100 & 70 & $<200$ & 200 & N & 300 & 50 & 500 & N & $>2,000$ \\
\hline LH1226C & 200 & N & N & 700 & 1,000 & $<20$ & 500 & 700 & N & $>2,000$ \\
\hline LH1227C & 200 & 50 & $<200$ & 1,000 & 1,000 & 30 & 1,000 & 700 & N & $>2,000$ \\
\hline LH1228C & 15 & N & 300 & N & N & 150 & N & 200 & N & $>2,000$ \\
\hline LH1229C & 15 & $N$ & 200 & $\mathbf{N}$ & N & 100 & 50 & 300 & N & $>2,000$ \\
\hline LH1230C & 10 & $N$ & N & N & N & 100 & 150 & 200 & N & $>2,000$ \\
\hline LH1231C & 70 & $\mathbf{N}$ & N & 1,500 & 3,000 & N & 150 & 700 & N & $>2,000$ \\
\hline LH1232C & 30 & 30 & 300 & N & N & 200 & N & 150 & N & $>2,000$ \\
\hline LH1233C & 15 & N & 200 & N & N & 200 & N & 30 & N & $>2,000$ \\
\hline
\end{tabular}




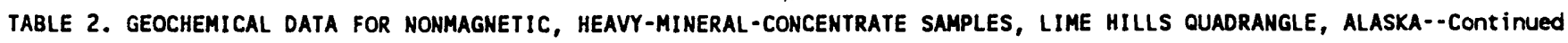

Sample Latitude Longitude Fe $\%$-s Mg $\%$-s $\quad$ Ca $\%$-s $\quad$ Na $\%$-s $\quad$ Ti $\%$-s $\quad p \quad \%$-s Ag ppm-s As ppm-s

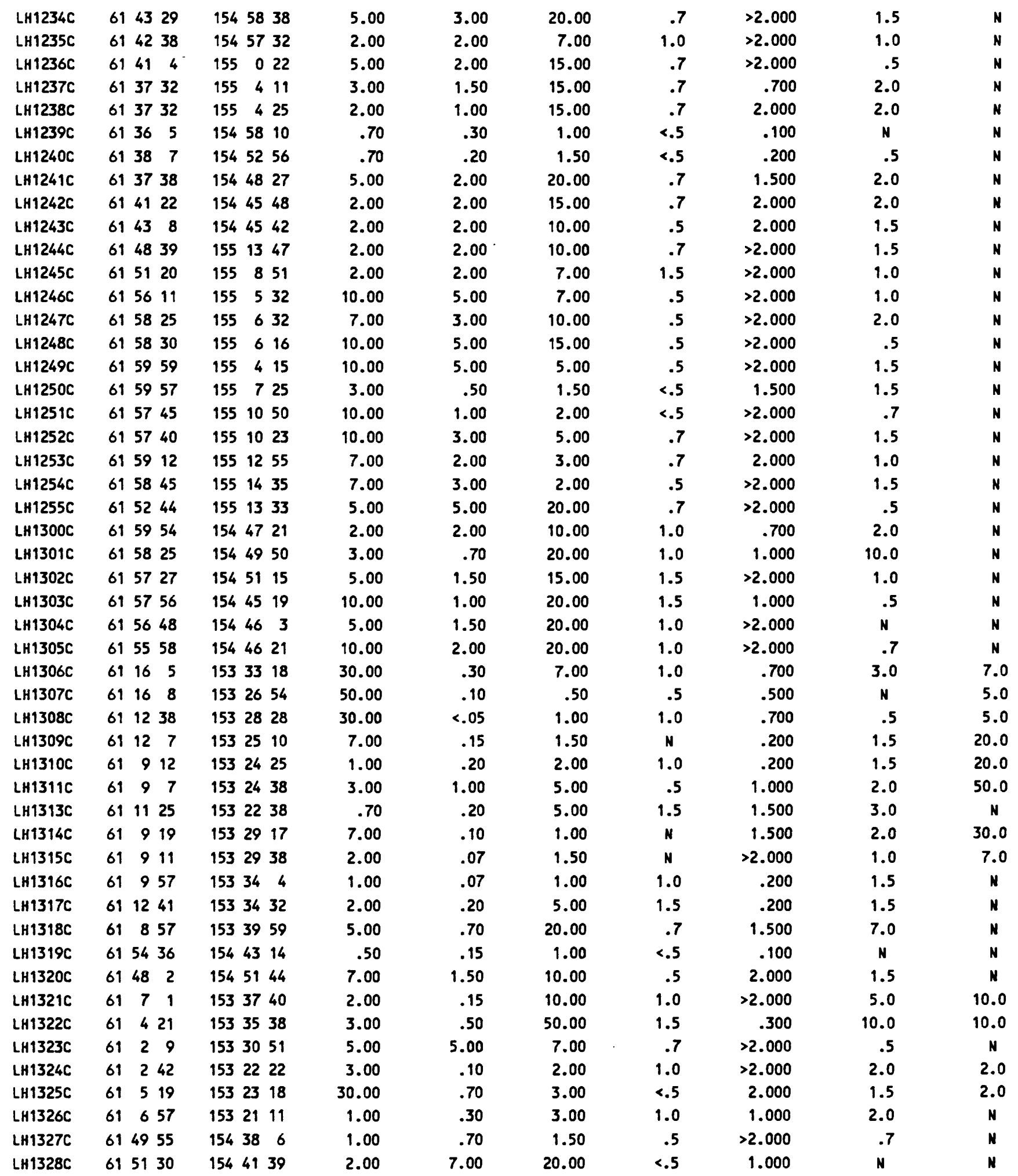




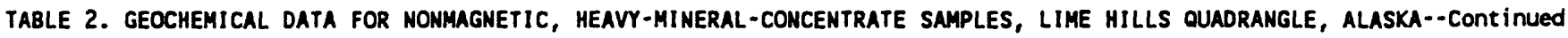
Sample AU ppm-s B ppm-s Ba ppm-s Be ppm-s $\quad$ Bi ppm-s Cd ppm-s Co ppm-s Cr ppm-s CU ppm-s

\begin{tabular}{|c|c|c|c|c|c|c|c|c|c|}
\hline LH1234C & $\mathbf{N}$ & 200 & 10,000 & 20 & $\mathbf{N}$ & N & 20 & 500 & 30 \\
\hline LH1235C & $\mathbf{N}$ & 70 & $>10,000$ & 2 & N & $N$ & $<20$ & 70 & 30 \\
\hline LH1236C & N & 100 & 5,000 & 3 & $N$ & $N$ & 30 & 100 & 70 \\
\hline LH1237C & N & 100 & $>10,000$ & 3 & $\mathbf{N}$ & $N$ & $<20$ & 150 & 10 \\
\hline LH1238C & N & 150 & $>10,000$ & 15 & $N$ & $N$ & $N$ & 70 & 20 \\
\hline LH1239C & $N$ & 20 & $>10,000$ & $N$ & N & $N$ & N & 30 & $<10$ \\
\hline LH1240C & $\mathbf{N}$ & $N$ & $>10,000$ & N & $N$ & $N$ & $\mathbf{N}$ & 20 & $<10$ \\
\hline LH1241C & N & 200 & 10,000 & 100 & $N$ & N & $<20$ & 100 & 50 \\
\hline LH1242C & $N$ & 100 & 7,000 & 30 & $N$ & $N$ & $<20$ & 100 & 10 \\
\hline LH1243C & $N$ & 200 & $>10,000$ & 50 & $N$ & $N$ & $<20$ & 100 & 10 \\
\hline LH1244C & $N$ & 200 & 3,000 & 30 & $N$ & $\mathbf{N}$ & 30 & 500 & 20 \\
\hline LH1245C & $N$ & $>5,000$ & $>10,000$ & 50 & N & $N$ & 20 & 300 & 30 \\
\hline LH $1246 \mathrm{C}$ & $N$ & 100 & 5,000 & $\mathbf{N}$ & $\mathbf{N}$ & $\mathbf{N}$ & 70 & 500 & 70 \\
\hline LH1247C & N & 150 & 1,500 & 3 & $\mathbf{N}$ & $\mathbf{N}$ & 50 & 300 & 150 \\
\hline LH1248C & $N$ & 200 & 1,000 & 2 & $N$ & N & 70 & 500 & 70 \\
\hline LH1249C & $\mathbf{N}$ & 150 & $>10,000$ & $\mathbf{N}$ & $\mathbf{N}$ & $\mathbf{N}$ & 50 & 200 & 50 \\
\hline LH1250C & $N$ & 30 & $>10,000$ & $N$ & $N$ & $N$ & $<20$ & 70 & 30 \\
\hline LH1251C & $N$ & 20 & $>10,000$ & $\mathbf{N}$ & $\mathbf{N}$ & $N$ & 20 & 70 & 50 \\
\hline LH1252C & $\mathbf{N}$ & 150 & $>10,000$ & $\mathbf{N}$ & $N$ & N & 50 & 150 & 70 \\
\hline LH1253C & $N$ & 70 & $>10,000$ & $\mathbf{N}$ & $\mathbf{N}$ & $<50$ & 30 & 100 & 70 \\
\hline LH1254C & $N$ & 500 & $>10,000$ & 2 & $N$ & $N$ & 30 & 700 & 70 \\
\hline LK1255C & $N$ & 500 & 3,000 & 5 & $\mathbf{N}$ & N & 30 & 700 & 20 \\
\hline LH130OC & $\mathbf{N}$ & 20 & $>10,000$ & $N$ & N & N & $<20$ & 70 & 10 \\
\hline L.H1301C & $\mathbf{N}$ & 50 & $>10,000$ & $N$ & $\mathbf{N}$ & $\mathbf{N}$ & $<20$ & 70 & 50 \\
\hline LH1302C & $\mathbf{N}$ & 700 & 10,000 & 3 & N & N & 20 & 300 & 50 \\
\hline LH1303C & N & 1,500 & 1,000 & 2 & $N$ & N & 50 & 100 & 30 \\
\hline LH1304C & $\mathbf{N}$ & 2,000 & 700 & 2 & N & N & 30 & 200 & 50 \\
\hline LH1305C & $\mathbf{N}$ & 1,500 & 1,500 & 2 & $\mathbf{N}$ & $N$ & 50 & 500 & 70 \\
\hline LH1306C & $\mathbf{N}$ & N & 300 & N & $\mathbf{N}$ & $\mathbf{N}$ & 300 & 20 & 150 \\
\hline LH1307C & $N$ & $N$ & 700 & $N$ & 50 & $\mathbf{N}$ & 200 & $<20$ & 100 \\
\hline LH1308C & $\mathbf{N}$ & $N$ & 2,000 & N & 150 & N & 100 & $<20$ & 70 \\
\hline LH1309C & N & $N$ & 1,000 & N & 2,000 & N & 100 & $<20$ & 100 \\
\hline LH1310C & 100 & $N$ & 7,000 & N & 300 & N & 300 & $<20$ & 10 \\
\hline LH1311C & N & 50 & 1,500 & N & N & $\mathbf{N}$ & 30 & 70 & 100 \\
\hline LH1313C & N & N & 300 & N & 150 & $\mathbf{N}$ & $N$ & $<20$ & $<10$ \\
\hline LH1314C & N & $N$ & 700 & N & 150 & N & 70 & $<20$ & 70 \\
\hline LH1315C & $\mathbf{N}$ & $\mathbf{N}$ & 1,000 & 2 & 150 & $\mathbf{N}$ & 50 & $<20$ & 100 \\
\hline LH1316C & N & $N$ & 1,500 & N & N & $\mathbf{N}$ & 30 & $<20$ & 10 \\
\hline LH1317C & N & N & 3,000 & N & N & N & 100 & $<20$ & 30 \\
\hline L.н1318C & N & 50 & 700 & $<2$ & N & $N$ & 70 & 50 & 100 \\
\hline LH1319C & $\mathbf{N}$ & N & $>10,000$ & 3 & $N$ & 100 & $N$ & $<20$ & 15 \\
\hline L.H1320C & $\mathbf{N}$ & 1,500 & $>10,000$ & $<2$ & $N$ & N & 50 & 100 & 70 \\
\hline LH1321C & $N$ & N & 5,000 & $N$ & 50 & $N$ & 30 & $<20$ & 70 \\
\hline LH1322C & N & 20 & 1,000 & $N$ & $N$ & N & 70 & $<20$ & 20 \\
\hline LH1323C & N & $\mathbf{N}$ & $>10,000$ & $N$ & $N$ & N & 150 & 70 & 100 \\
\hline LH1324C & $\mathbf{N}$ & $\mathbf{N}$ & 2,000 & N & 70 & $\mathbf{N}$ & 70 & $<20$ & 70 \\
\hline LH1325C & $\mathbf{N}$ & $\mathbf{N}$ & 1,000 & N & $N$ & $\mathbf{N}$ & 700 & 150 & 500 \\
\hline LH1326C & N & 30 & 300 & 2 & 150 & $N$ & 20 & $<20$ & 70 \\
\hline LH1327C & $N$ & 300 & 700 & 3 & N & $\mathbf{N}$ & $N$ & 100 & 10 \\
\hline LH1328C & N & 200 & 3,000 & 3 & $\mathbf{N}$ & $\mathbf{N}$ & $<20$ & 100 & $<10$ \\
\hline
\end{tabular}


TABLE 2. GEOCHEMICAL DATA FOR NOMMAGNETIC, hEAVY-MINERAL-CONCENTRATE SAMPLES, LIME HILLS QUADRANGLE, ALASKA--CONTInUEd Sample Ga ppm-s Ge ppm-s La ppm-s Mn ppm-s Mo ppm-s Nb ppm-s Ni ppm-s Pb ppm-s Pt ppm-s Sb ppm-s

\begin{tabular}{|c|c|c|c|c|c|c|c|c|c|}
\hline LH1234C & 30 & $N$ & N & 700 & $N$ & 50 & 50 & $<20$ & N \\
\hline LH1235C & 15 & $N$ & $N$ & 500 & N & $<50$ & 15 & $<20$ & N \\
\hline LH1236C & 20 & $N$ & $N$ & 700 & $N$ & $<50$ & 30 & $<20$ & N \\
\hline LH1237C & 10 & $N$ & $N$ & 500 & $N$ & $<50$ & 50 & $<20$ & $N$ \\
\hline LH1238C & 15 & $N$ & 150 & 500 & $N$ & $<50$ & 20 & N & $\mathbf{N}$ \\
\hline LH1239C & $N$ & $N$ & $N$ & 100 & $N$ & $<50$ & 10 & $N$ & $N$ \\
\hline LH $1240 \mathrm{C}$ & $<10$ & $N$ & N & 300 & N & $<50$ & 10 & $N$ & N \\
\hline LH1241C & 15 & $N$ & 100 & 700 & $N$ & 70 & 20 & $N$ & N \\
\hline LH1242C & 15 & $N$ & $M$ & 1,000 & $M$ & 50 & 10 & $N$ & N \\
\hline LH1243C & 10 & $N$ & N & 700 & $N$ & 50 & 15 & $N$ & N \\
\hline LH1244C & 10 & $N$ & 150 & 500 & $N$ & 200 & 70 & $N$ & N \\
\hline LH1245C & 15 & $N$ & 100 & 500 & $N$ & 100 & 70 & $N$ & N \\
\hline LH1246C & 10 & $N$ & 300 & 2,000 & $N$ & 100 & 70 & 30 & N \\
\hline LH1247C & 10 & $N$ & 500 & 1,500 & $N$ & 100 & 70 & 30 & N \\
\hline LH1248C & 15 & $N$ & 700 & 3,000 & $N$ & 70 & 100 & 30 & $N$ \\
\hline LH1249C & 15 & $N$ & $>2,000$ & 2,000 & N & 50 & 50 & 20 & N \\
\hline LH1250C & N & N & 150 & 300 & N & $<50$ & 20 & $N$ & N \\
\hline LH1251C & $<10$ & $N$ & 150 & 500 & 20 & 50 & 30 & $<20$ & N \\
\hline LH1252C & 10 & $N$ & $>2,000$ & 2,000 & $N$ & 50 & 50 & 30 & N \\
\hline LH1253C & $<10$ & $N$ & 700 & 1,000 & $N$ & $<50$ & 50 & 20 & H \\
\hline LH1254C & $<10$ & N & 700 & 2,000 & $N$ & 70 & 50 & 20 & N \\
\hline LH1255C & 10 & $N$ & 150 & 1,000 & $\mathbf{N}$ & 150 & 100 & $<20$ & N \\
\hline LH1300C & $<10$ & N & $<100$ & 1,000 & $\mathbf{N}$ & $<50$ & 20 & N & N \\
\hline LH1301C & $<10$ & $N$ & 100 & 300 & $M$ & 50 & 70 & 20 & N \\
\hline LH1302C & 20 & N & N & 700 & $N$ & $<50$ & 50 & 20 & N \\
\hline LH1303C & 20 & H & H & 500 & $<10$ & 70 & 70 & 30 & N \\
\hline LH1304C & 20 & N & N & 700 & N & 50 & 50 & $<20$ & $N$ \\
\hline LH1305C & 15 & N & 200 & 700 & $\mathbf{N}$ & $<50$ & 150 & 30 & N \\
\hline LH1306C & $<10$ & $M$ & 200 & 200 & 20 & $<50$ & 300 & 500 & N \\
\hline LH1307C & $<10$ & M & 150 & 200 & N & 50 & 30 & 300 & N \\
\hline LH1308C & $<10$ & $N$ & $N$ & 100 & 20 & 50 & 15 & 150 & $N$ \\
\hline LH1309C & $<10$ & $N$ & 150 & 200 & 300 & $<50$ & 15 & 200 & $N$ \\
\hline LH1310C & $<10$ & $N$ & 100 & 300 & 20 & 70 & 20 & 20 & $N$ \\
\hline LH1311C & 10 & $N$ & 200 & 1,000 & 150 & 70 & 50 & 500 & $N$ \\
\hline LH1313C & 10 & $N$ & 150 & 500 & N & $<50$ & $<10$ & 20 & N \\
\hline LH1314C & $<10$ & $N$ & 700 & 500 & 30 & $<50$ & $<10$ & 1,000 & N \\
\hline LH1315C & $N$ & $N$ & $>2,000$ & 1,000 & 700 & 200 & N & 150 & N \\
\hline LH1316C & 10 & $N$ & 150 & 300 & 15 & $<50$ & $<10$ & 100 & N \\
\hline LH1317C & 15 & $N$ & 150 & 500 & 50 & $<50$ & 20 & 700 & N \\
\hline LH1318C & 20 & $N$ & 150 & 3,000 & $N$ & $<50$ & 20 & $<20$ & N \\
\hline LH1319C & N & N & $N$ & 50 & $<10$ & $<50$ & $<10$ & $N$ & N \\
\hline LH1320C & 10 & $\mathbf{N}$ & 100 & 700 & N & 50 & 50 & 100 & N \\
\hline LH1321C & 10 & $N$ & 200 & 500 & 10 & 50 & $<10$ & 100 & $N$ \\
\hline LH1322C & 15 & $N$ & 300 & 1,500 & 10 & $<50$ & 15 & $<20$ & $N$ \\
\hline LH1323C & $<10$ & $N$ & N & 1,000 & N & 50 & 70 & 50 & N \\
\hline LH1324C & $<10$ & $N$ & 200 & 300 & 10 & 70 & 10 & 30 & $N$ \\
\hline LH1325C & 10 & $N$ & N & 200 & N & $<50$ & 700 & 700 & N \\
\hline LH1326C & 10 & $N$ & 500 & 500 & N & 50 & $<10$ & 50 & $\mathbf{N}$ \\
\hline LH1327C & $<10$ & $\mathbf{N}$ & 300 & 500 & N & 150 & $<10$ & 20 & $N$ \\
\hline LH1328C & 15 & N & $N$ & 700 & $\mathbf{N}$ & 50 & 10 & $<20$ & $\mathbf{N}$ \\
\hline
\end{tabular}


TABLE 2. GEOCHEMICAL DATA FOR NONMAGNETIC, hEAVY-MINERAL-CONCENTRATE SAMPLES, LIME hILLS QUADRANGLE, ALASKA--CONTINUEd

Sample Sc ppm-s Sn ppm-s Sr ppm-s Th ppm-s $U$ ppm-s $V$ ppm-s $W$ ppm-s $\quad$ Y ppm-s $2 n$ ppm-s $2 r$ ppm-s

\begin{tabular}{|c|c|c|c|c|c|c|c|c|c|c|}
\hline LH1234C & 20 & 50 & 1,000 & N & N & 200 & $N$ & 150 & $N$ & $>2,000$ \\
\hline LH1235C & 15 & 700 & 1,000 & N & $\mathbf{N}$ & 150 & 50 & 200 & $N$ & $>2,000$ \\
\hline LH1236C & 15 & 150 & 700 & $N$ & $\mathbf{N}$ & 300 & 70 & 100 & $\mathbf{N}$ & $>2,000$ \\
\hline LH1237C & 15 & 1,000 & 2,000 & $N$ & $N$ & 150 & 50 & 200 & $N$ & $>2,000$ \\
\hline LH1238C & 15 & 70 & 1,500 & $N$ & N & 150 & $N$ & 200 & $N$ & $>2,000$ \\
\hline LH1239C & $N$ & N & 7,000 & N & N & 20 & $\mathbf{N}$ & 70 & N & $>2,000$ \\
\hline LH1240C & $<10$ & 700 & 7,000 & $<200$ & $N$ & 20 & 50 & 200 & N & N \\
\hline LH1241C & 20 & 700 & 1,000 & $<200$ & N & 150 & 70 & 300 & $N$ & N \\
\hline LH1242C & 30 & 1,500 & 1,000 & 500 & $<1,000$ & 150 & 300 & 500 & $N$ & $N$ \\
\hline LH1243C & 50 & 1,500 & 1,000 & 700 & 1,000 & 150 & 200 & 700 & $N$ & N \\
\hline LH1244C & 100 & 70 & 500 & N & N & 700 & N & 100 & N & 2,000 \\
\hline LH1245C & 30 & $N$ & 300 & $N$ & $\mathbf{N}$ & 200 & $N$ & 70 & $N$ & 2,000 \\
\hline LH1246C & 70 & N & 200 & $\mathbf{N}$ & $\mathbf{N}$ & 300 & $N$ & 100 & $\mathbf{N}$ & N \\
\hline LH1247C & 70 & 200 & 200 & $N$ & $N$ & 500 & $N$ & 200 & $\mathbf{N}$ & N \\
\hline LH1248C & 70 & $N$ & 200 & N & $N$ & 300 & $N$ & 150 & $N$ & N \\
\hline LH1249C & 150 & 50 & 200 & $<200$ & $\mathbf{N}$ & 300 & $N$ & 300 & $N$ & $N$ \\
\hline LH1250C & 10 & $N$ & 1,000 & N & $N$ & 100 & $N$ & 70 & $N$ & 1,500 \\
\hline LH1251C & 15 & N & 100 & N & $N$ & 150 & N & 50 & $N$ & 1,500 \\
\hline LH1252C & 50 & N & 200 & $N$ & $N$ & 300 & $N$ & 150 & N & N \\
\hline LH1253C & 30 & N & 700 & $N$ & $N$ & 150 & $N$ & 100 & 2,000 & $N$ \\
\hline LH1254C & 70 & 300 & 200 & $N$ & $N$ & 200 & $N$ & 200 & N & N \\
\hline LH1255C & 100 & 20 & 200 & $\dot{N}$ & N & 500 & $N$ & 100 & $N$ & N \\
\hline LH1300C & 10 & N & 2,000 & $N$ & N & 150 & 50 & 150 & $N$ & $>2,000$ \\
\hline LH1301C & 15 & 200 & 3,000 & $N$ & $N$ & 150 & $N$ & 200 & $N$ & $>2,000$ \\
\hline LH $1302 \mathrm{C}$ & 15 & 200 & 700 & $N$ & $N$ & 150 & 50 & 150 & $N$ & $>2,000$ \\
\hline LH1303C & 15 & 700 & 700 & 300 & N & 150 & 100 & 150 & 500 & $>2,000$ \\
\hline LH1304C & 15 & 30 & 500 & N & $N$ & 500 & N & 100 & $N$ & $>2,000$ \\
\hline LH1305C & 20 & $N$ & 700 & $N$ & $N$ & 300 & 50 & 200 & N & $>2,000$ \\
\hline LH1306C & 10 & $N$ & 200 & $<200$ & $N$ & 100 & 200 & 150 & N & $>2,000$ \\
\hline LH1307C & 15 & $N$ & N & $N$ & $N$ & 30 & $N$ & 200 & 1,500 & $>2,000$ \\
\hline LH1308C & 30 & 70 & $<200$ & $\mathbf{N}$ & N & 30 & 200 & 500 & N & $>2,000$ \\
\hline LH1309C & 100 & 700 & $N$ & 500 & 1,500 & 30 & 7,000 & 1,000 & $N$ & $>2,000$ \\
\hline LH1310C & 50 & $N$ & $<200$ & N & N & 20 & 700 & 700 & $N$ & $>2,000$ \\
\hline LH1311C & 20 & $N$ & $<200$ & 2,000 & 1,000 & 100 & 5,000 & 500 & $N$ & $>2,000$ \\
\hline LH1313C & 20 & $N$ & 200 & N & N & 100 & 150 & 300 & $N$ & $>2,000$ \\
\hline LH1314C & 70 & $\mathbf{N}$ & N & 1,500 & 2,000 & 20 & 70 & 700 & $N$ & $>2,000$ \\
\hline LH1315C & 200 & 70 & $\mathbf{N}$ & $>5,000$ & 15,000 & 100 & 5,000 & 5,000 & $\mathbf{N}$ & $>2,000$ \\
\hline LH1316C & 50 & N & $<200$ & 700 & 1,500 & 20 & 300 & 500 & N & $>2,000$ \\
\hline LH1317C & 30 & N & $<200$ & 500 & N & 30 & 150 & 500 & $\mathbf{N}$ & $>2,000$ \\
\hline LH1318C & 20 & $N$ & $<200$ & N & N & 150 & 300 & 200 & N & $>2,000$ \\
\hline LH1319C & $N$ & 700 & 3,000 & N & $N$ & N & N & 20 & 3,000 & 2,000 \\
\hline LH1320C & 20 & 100 & 700 & N & $N$ & 150 & 100 & 300 & $N$ & $>2,000$ \\
\hline LH1321C & 30 & $>2,000$ & $<200$ & 500 & $<1,000$ & 70 & 300 & 500 & $N$ & $>2,000$ \\
\hline LH1322C & 10 & 500 & 500 & $N$ & N & 70 & 500 & 300 & N & $>2,000$ \\
\hline LH1323C & 15 & 150 & 300 & $N$ & N & 100 & 70 & 200 & N & $>2,000$ \\
\hline LH1324C & 30 & 500 & $<200$ & 700 & $<1,000$ & 100 & 300 & 300 & $N$ & $>2,000$ \\
\hline LH1325C & 15 & 30 & N & $N$ & N & 70 & 100 & 150 & N & $>2,000$ \\
\hline LH1326C & 20 & 300 & $<200$ & 200 & $N$ & 30 & 150 & 700 & $\mathbf{N}$ & $>2,000$ \\
\hline LH1327C & 20 & 150 & $<200$ & 300 & $\mathbf{N}$ & 70 & 50 & 500 & $\mathbf{N}$ & $>2,000$ \\
\hline LH1328C & 15 & N & 200 & N & $\mathbf{N}$ & 150 & $N$ & 300 & $N$ & $>2,000$ \\
\hline
\end{tabular}


TABLE 2. GEOCHEMICAL DATA FOR NOMMAGNETIC, hEAVY-MINERAL-CONCENTRATE SAMPLES, LIME hILLS QUAdRAMGLE, ALASKA--ContinUed

\begin{tabular}{|c|c|c|c|c|c|c|c|c|c|}
\hline Sample & Latitude & Longi tude & $x-s$ & $x-s$ & $x-s$ & $x-s$ & $x-s$ & X-s & Ag ppm-s \\
\hline LH1329C & $\begin{array}{lll}61557 & 7\end{array}$ & 1545437 & 7.00 & 3.00 & 15.00 & .7 & $>2.000$ & $N$ & N \\
\hline LH1330C & 61583 & $15458 \quad 22$ & 5.00 & 1.50 & 10.00 & $<.5$ & $>2.000$ & 2.0 & N \\
\hline LH1331C & 615753 & 154589 & 2.00 & 3.00 & 20.00 & .7 & $>2.000$ & 1.0 & N \\
\hline LH1332C & 615358 & 155218 & 2.00 & 3.00 & 20.00 & 1.0 & $>2.000$ & 1.0 & $N$ \\
\hline LH1333C & 615332 & 155741 & 5.00 & 3.00 & 10.00 & .5 & $>2.000$ & 2.0 & $n$ \\
\hline LH1334C & 615312 & 1545932 & 1.50 & .50 & 5.00 & $<.5$ & .500 & .7 & N \\
\hline LH1335C & 614749 & 155820 & 2.00 & 1.00 & 20.00 & .7 & $>2.000$ & 5.0 & $\mathbf{N}$ \\
\hline LH1336C & 614624 & $155 \quad 10 \quad 4$ & .70 & .30 & 1.50 & $<.5$ & .700 & .7 & $N$ \\
\hline LH1337C & 614455 & 1545554 & 7.00 & 2.00 & 10.00 & .7 & $>2.000$ & .7 & $N$ \\
\hline LH1338C & 614628 & 1545411 & 5.00 & 1.50 & 10.00 & .7 & 2.000 & 1.5 & N \\
\hline LH1339C & 614233 & 1545934 & 5.00 & $2.00^{\circ}$ & 15.00 & 1.5 & 2.000 & 1.0 & N \\
\hline LH1340C & 61393 & 1545916 & 1.00 & 1.00 & 10.00 & .7 & 1.000 & 1.0 & $\mathbf{N}$ \\
\hline LH1341C & $61 \quad 40 \quad 12$ & $154 \quad 4751$ & 1.50 & 1.00 & 15.00 & .7 & 2.000 & 2.0 & N \\
\hline LH1343C & 614336 & $15450 \quad 0$ & .70 & .50 & 2.00 & $<.5$ & .300 & .5 & N \\
\hline
\end{tabular}


TABLE 2. GEOCHEMICAL DATA FOR NONMAGNETIC, hEAVY-MIMERAL-CONCENTRATE SAMPLES, LIME hILLS QUADRANGLE, ALASKA--CONT inUed Sample AU ppm-s B ppm-s Ba ppm-s Be ppm-s Bi ppm-s $\quad$ cd ppm-s co ppm-s Cr ppm-s CU ppm-s

\begin{tabular}{|c|c|c|c|c|c|c|c|c|}
\hline LH1329C & N & 200 & 1,500 & 2 & $N$ & $N$ & 70 & 1,000 \\
\hline LH1330C & N & 100 & 7,000 & 3 & $N$ & $<50$ & 30 & 700 \\
\hline LH1331C & N & 1,000 & 7,000 & 7 & N & N & 30 & 500 \\
\hline LH1332C & $N$ & 1,500 & 5,000 & 10 & N & $N$ & 30 & 500 \\
\hline LH1333C & $N$ & 300 & 3,000 & 2 & N & N & 30 & 500 \\
\hline LH1334C & N & 150 & $>10,000$ & N & N & N & N & 50 \\
\hline LH1335C & $N$ & 50 & $>10,000$ & 2 & N & $<50$ & 20 & 150 \\
\hline LH1336C & $N$ & N & $>10,000$ & N & N & N & N & $<20$ \\
\hline LH1337C & N & 200 & 5,000 & 2 & N & N & 50 & 300 \\
\hline LH1338C & $N$ & 200 & $>10,000$ & $<2$ & N & N & 30 & 100 \\
\hline LH1339C & N & 150 & 10,000 & 5 & N & N & 20 & 200 \\
\hline LH1340C & N & 100 & $>10,000$ & 7 & N & $N$ & $<20$ & 200 \\
\hline LH1341C & N & 100 & 10,000 & 7 & 200 & N & $<20$ & 200 \\
\hline LH1343C & $N$ & 30 & $>10,000$ & N & $N$ & N & N & 30 \\
\hline
\end{tabular}


TABLE 2. GEOCHEMICAL DATA FOR NONMAGNETIC, hEAVY-MINERAL-CONCENTRATE SAMPLES, LIME hILLS QUADRANGLE, ALASKA--CONTInUEd

Sample Ga ppm-s Ge ppm-s La ppm-s Mn ppm-s Mo ppm-s Nb ppm-s Ni ppm-s Pb ppm-s Pt ppm-s Sb ppm-s

\begin{tabular}{|c|c|c|c|c|c|c|c|c|c|}
\hline LH1329C & 15 & N & $\boldsymbol{H}$ & 700 & N & $<50$ & 100 & 20 & N \\
\hline LH1330C & $<10$ & N & 300 & 500 & $N$ & 100 & 70 & $<20$ & $N$ \\
\hline LH1331C & $<10$ & N & 100 & 500 & N & 150 & 70 & N & $N$ \\
\hline LH1332C & 10 & $N$ & 100 & 500 & $N$ & 150 & 70 & N & $N$ \\
\hline LH1333C & 10 & N & 200 & 1,500 & N & 70 & 70 & $<20$ & N \\
\hline LH1334C & $<10$ & N & $N$ & 70 & N & $<50$ & 15 & $N$ & N \\
\hline LH1335C & 10 & N & 100 & 300 & N & 100 & 30 & $<20$ & N \\
\hline LH1336C & $<10$ & N & N & 100 & N & $<50$ & $<10$ & $N$ & N \\
\hline LH1337C & 20 & N & 200 & 1.000 & N & 70 & 70 & 30 & N \\
\hline LH1338C & 15 & N & N & 700 & 150 & $<50$ & 30 & 30 & N \\
\hline LH1339C & 20 & $N$ & 150 & 500 & $N$ & 50 & 30 & 20 & $N$ \\
\hline LH1340C & $<10$ & N & $<100$ & 500 & $\mathbf{N}$ & $<50$ & 20 & $N$ & N \\
\hline LH1341C & 10 & $N$ & 100 & 500 & 20 & 70 & 10 & 20 & N \\
\hline LH1343C & $<10$ & N & $N$ & 150 & $N$ & $<50$ & $<10$ & N & N \\
\hline
\end{tabular}




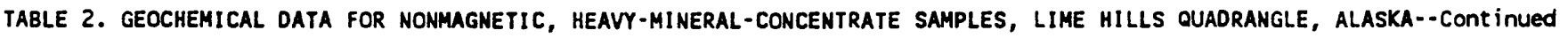

\begin{tabular}{|c|c|c|c|c|c|c|c|c|c|c|c|c|c|c|}
\hline Sample & sc ppm-s & Sn ppm-s & Sr ppm-s & Th ppon-s & $U$ & ppm-s & $v$ & ppm-s & $W$ & ppm-s & $Y$ & ppm-s & Zn ppm-s & Zr ppm-s \\
\hline LH1329C & 30 & $N$ & 700 & $<200$ & & N & & 200 & & $N$ & & 100 & N & $>2,000$ \\
\hline LH1330C & 30 & 50 & 500 & N & & N & & 300 & & N & & 150 & 1,000 & $>2,000$ \\
\hline LH1331C & 70 & 500 & 300 & $<200$ & & N & & 300 & & 50 & & 150 & N & $>2,000$ \\
\hline LH1332C & 70 & 30 & 300 & N & & N & & 300 & & $\mathbf{N}$ & & 100 & $N$ & $>2,000$ \\
\hline Lн1333С & 50 & 150 & 300 & N & & N & & 150 & & $N$ & & 150 & $\mathbf{N}$ & $>2,000$ \\
\hline LH1334C & N & N & 2,000 & N & & N & & 30 & & $\mathbf{N}$ & & 50 & 500 & 1,500 \\
\hline LH1335C & 50 & 50 & 1,500 & $\mathbf{N}$ & & N & & 300 & & $N$ & & 150 & 1.000 & $>2,000$ \\
\hline LH1336C & $<10$ & $N$ & 3,000 & $N$ & & $N$ & & 20 & & $N$ & & 50 & N & $>2,000$ \\
\hline LH1337C & 30 & 200 & 500 & $\mathbf{N}$ & & $N$ & & 500 & & $\mathbf{N}$ & & 100 & N & $>2,000$ \\
\hline LH1338C & 15 & N & 700 & $N$ & & N & & 150 & & $\mathbf{N}$ & & 100 & $\mathbf{N}$ & $>2,000$ \\
\hline LH1339C & 20 & 500 & 700 & N & & N & & 150 & & $N$ & & 300 & N & $>2,000$ \\
\hline LH1340C & 15 & 200 & 2,000 & N & & N & & 100 & & 70 & & 200 & $N$ & $>2,000$ \\
\hline LH1341C & 50 & $>2,000$ & 500 & 700 & & 1,500 & & 150 & & 2,000 & & 500 & N & $>2,000$ \\
\hline LH1343C & $<10$ & 50 & 5,000 & N & & $N$ & & 30 & & N & & 100 & N & $>2,000$ \\
\hline
\end{tabular}

